\title{
Development of a Protecting Group for Sulfate Esters.
}

Andrew D. Proud.

$\mathrm{PhD}$.

The University of Edinburgh.

1999

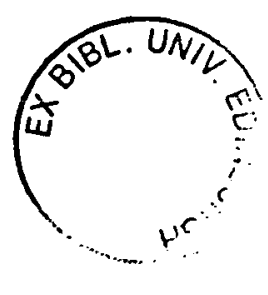




\section{Abstract:}

This work describes the development of a protecting group for sulfate monoesters in carbohydrates. After initial trial studies, the trifluoroethyl ester was selected as a suitable protecting group. The ester was formed from the sulfate by treatment with trifluorodiazoethane. This protection method is shown to be compatible with other common protecting groups used in carbohydrate chemistry. The protecting group is proven to be stable to the manipulations of many other protecting groups. The utility of monosaccharides incorporating protected sulfate esters in synthesis has been examined. It has been proven that they are useful and versatile building blocks in the synthesis of more complex structures. Selective cleavage of the trifluoroethyl ester was achieved with potassium tert-butoxide. The mechanism for this deprotection reaction has been studied and this investigation has led to the advancement of alternative deprotection methods.

I declare that, this Thesis has been composed by myself and that the work is my own.

Andrew David Proud. 


\section{Acknowledgements:}

I would like to thank my supervisor Prof. Sabine Flitsch for her help and support throughout this project. I also wish to thank Dr. Jeremy Prodger, of GlaxoWellcome and Prof. Nick Turner of The University of Edinburgh for their many suggestions and useful advice.

I wish to acknowledge the EPSRC and GlaxoWellcome for financial support.

I wish to thank all the people that run the NMR and X-ray crystallography services at Edinburgh University but especially John Miller and Simon Parsons.

I wish to thank all those that have worked in the research groups of Prof. Flitsch, Prof. Turner and Dr. Hume, who over the last three years have become great friends and from whom I have learned so much.

I thank my family for encouraging me in this continuation of my studies and for helping me realise that there are bigger things in life than this project.

And finally I wish to thank my wife Louise for her love and care and support. This $\mathrm{PhD}$ project has been part of our journey together where will we go next? 
"It is not always realised how widespread is the occurrence of carbohydrate sulphates in Nature."

E. G. V. Percival, Quart. Rev. (1949).

“Twa birds sat on a bara, one was a spug,

the other a spara."

By Wullie Shakespeare. 


\section{Table of Contents.}

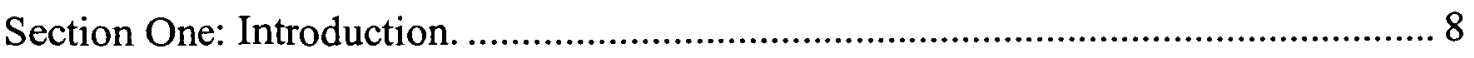

1. Biological Background to Carbohydrates. .................................................... 9

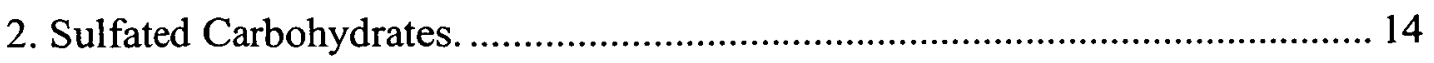

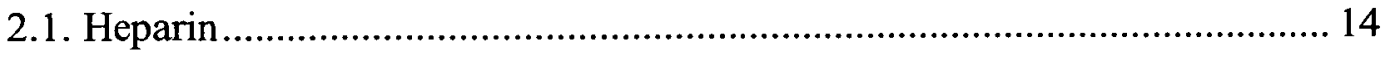

2.2. Nod Factors ............................................................................ 17

2.3. Sialyl Lewis Antigens ................................................................. 20

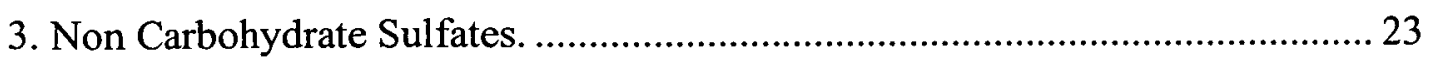

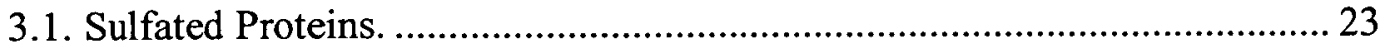

3.2. Sulfated Marine Natural Products.......................................................... 24

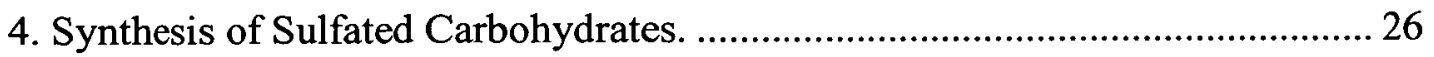

4.1. Sulfotransferase Enzymes. ......................................................... 26

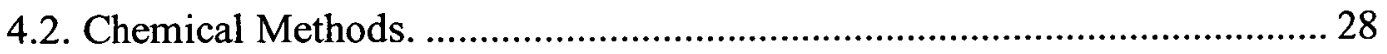

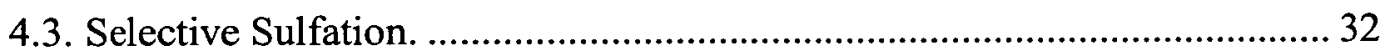

4.4. The Problem with Synthesis. ............................................................. 35

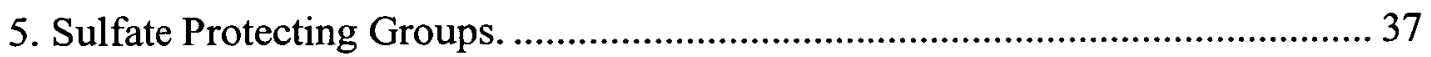

5.1. Requirements of a Sulfate Protecting Group. ...................................... 37

5.2. Development of a Sulfate Protecting Group. .......................................... 38

5.3. History of Sulfate Protecting Groups.................................................. 41

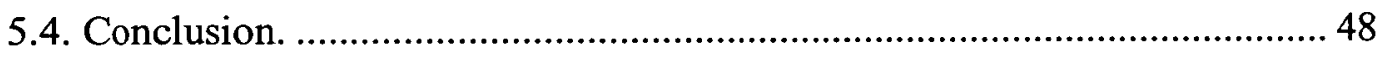

Section Two: Results and Discussion. .......................................................... 49

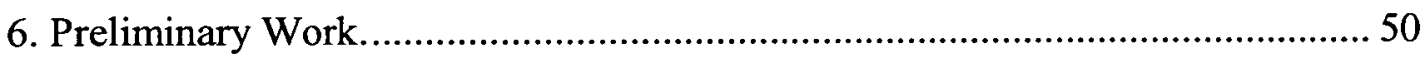

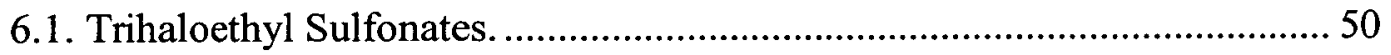

7. Development of a Synthesis of Sulfate Diesters. ......................................... 53

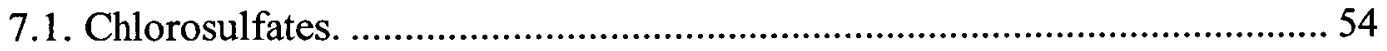

7.2. Trifluoroethyl Chlorosulfate. ............................................................ 58

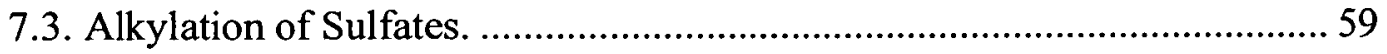

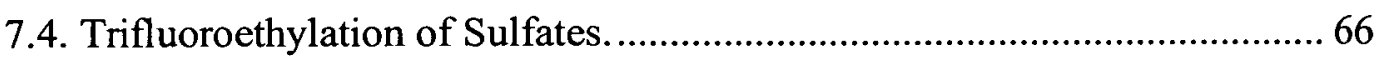

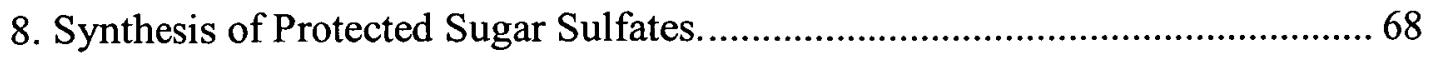

8.1. Protection of Sulfated Monosaccharides.............................................6 68 


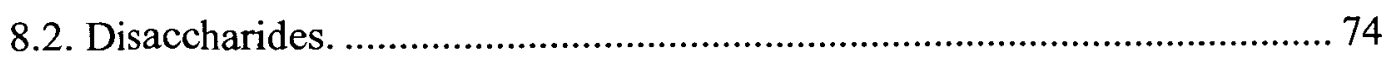

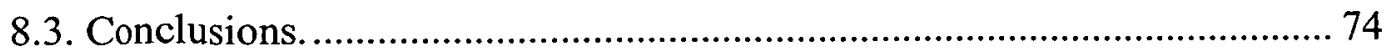

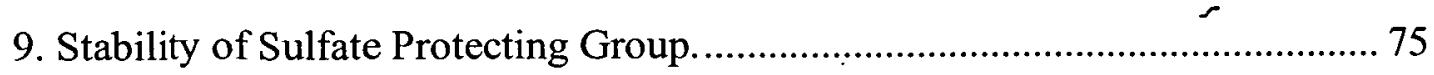

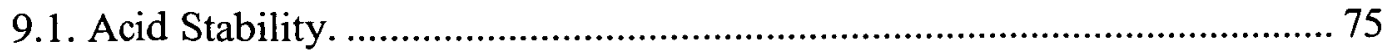

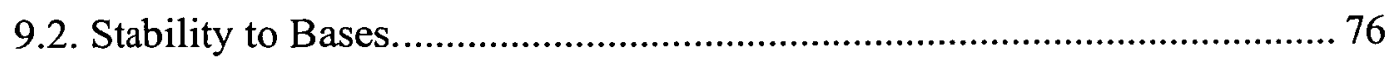

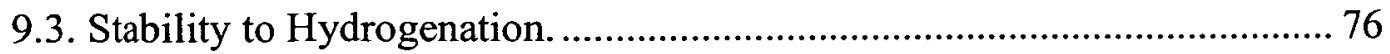

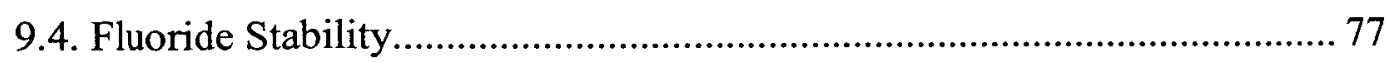

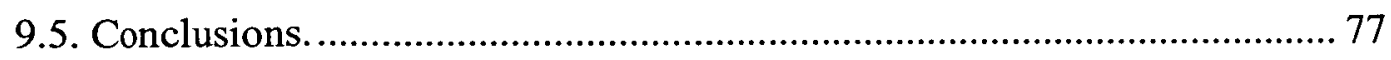

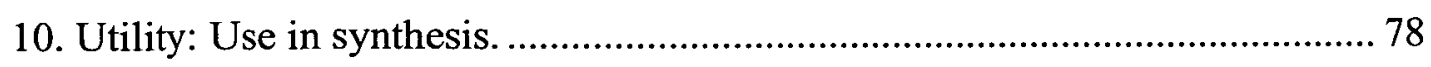

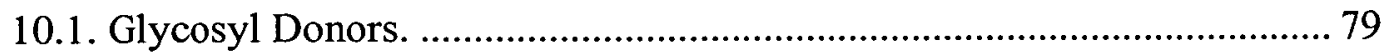

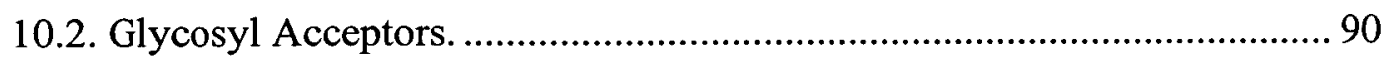

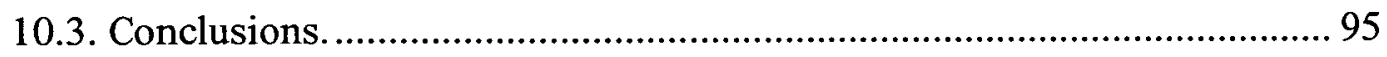

11. Deprotection of Protected Sulfate Diesters............................................... 96

11.1. Deprotection using Sodium Methoxide. ........................................... 97

11.2. Other Bases for the Deprotection of Trifluoroethyl Esters. ..................... 99

11.3. Potassium tert-Butoxide for the Deprotection of Trifluoroethyl Esters.. 101

11.4. Other Attempts at Deprotection Conditions.......................................... 103

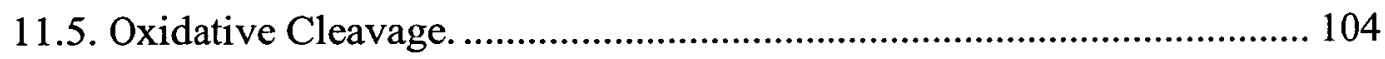

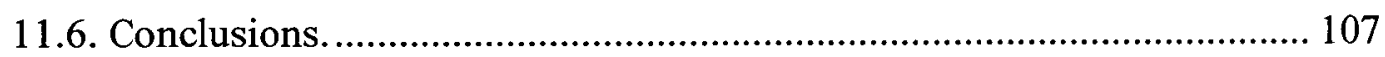

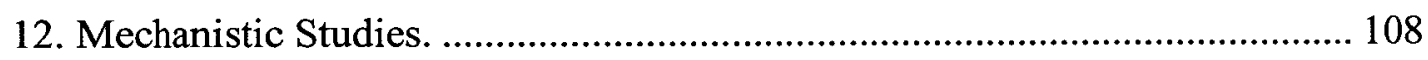

12.1. Simple Nucleophilic Displacements. ............................................ 108

12.2. Mechanisms Involving Multiple Steps. .......................................... 112

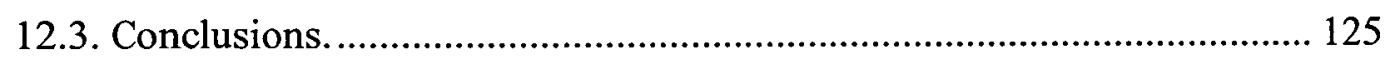

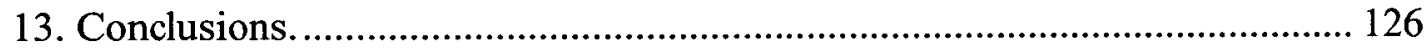

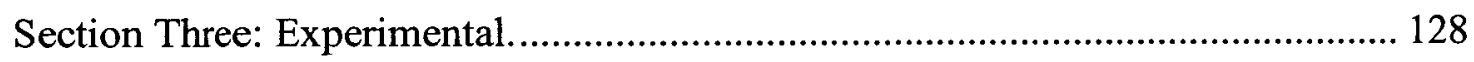

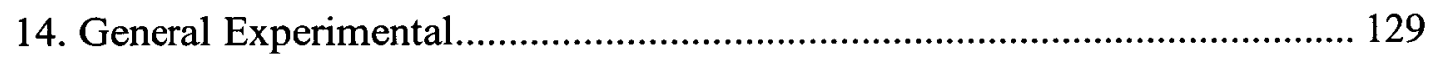

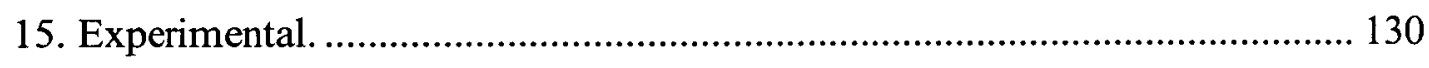

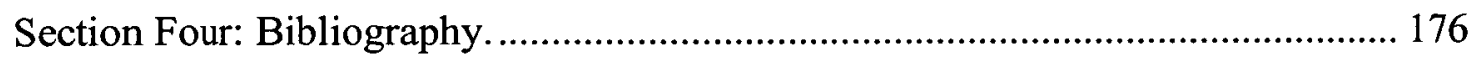

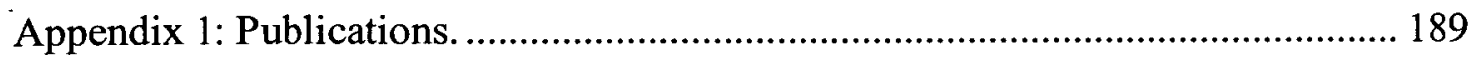

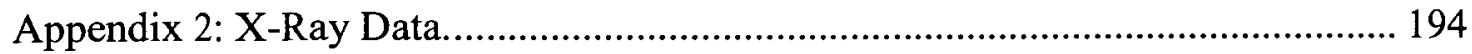




\section{Abbreviations:}

The use of abbreviations has been kept to a minimum throughout this thesis however the following have been used within the text:-

DCC Dicyclohexylcarbodiimide.

DCM

Dichloromethane.

DMAP

$N, N$-Dimethylaminopyridine.

DMF

Dimethyl formamide.

IR

Infra Red Spectroscopy.

LCMS

Liquid Chromatography Mass Spectrometry.

MS

Mass Spectrometry.

NBS

$N$-Bromosuccinimide.

NIS

$N$-Iodosuccinimide.

NMR

Nuclear Magnetic Resonance Spectroscopy.

TLC

Thin Layer Chromatography.

THF

Tetrahydrofuran.

UV

Ultra Violet. 
Section One: Introduction. 
This thesis project forms part of the ongoing research of Professor Sabine Flitsch at The University of Edinburgh into the development of methods for the synthesis of complex oligosaccharides. This work examines chemical and enzymatic routes to naturally occurring oligosaccharides which have uncommon constituent monosaccharides, unusual linkages or interesting additional functional groups.

This project is aimed at the development of methods for the synthesis of sulfated oligosaccharides and has been specifically focused on the invention of a protecting group for sulfate monoesters. Such an invention will greatly aid further synthetic projects.

The objective of this section is to introduce a number of important and interesting sulfated oligosaccharides and through a discussion of their biology, to make some justification for the interest in such compounds. This brief biological introduction is covered in Chapters 1,2 and 3. Chapter 4 discusses the various methods used to synthesis sulfated carbohydrates and why these methods are often inadequate. The section then discusses the advantages a sulfate protecting group would bring to a synthesis of sulfated compounds in Chapter 5 , followed by a short explanation of the properties that such a group would need. The introductory section finishes with a short discussion of the strategy adopted in this thesis project and the problems that were foreseen.

\section{Biological Background to Carbohydrates.}

This section discusses the importance of carbohydrates in biology and explains the background into which the work on sulfated carbohydrates fit.

The history of carbohydrate chemistry stretches back almost to the beginnings of organic chemistry. Studies of saccharides are partly responsible for modern understanding of chirality and stereochemistry and historical figures in carbohydrate 
chemistry have had a pronounced influence on our current concept of molecular structure in 3-dimensional space. However the area of carbohydrate chemistry has undergone a renaissance in the last two decades due in part to the advances in glycobiology.

It was not until the 1970's that the role of carbohydrates in nature was found to extend beyond the structural role that polysaccharides, such as cellulose and starch, play in plants. Up until this time it was known that carbohydrates played an essential part in energy storage and transport but they were not thought to have any real role in biological processes.

\begin{abstract}
"Polysaccharides seem at the moment to be less spectacular substances than proteins and nucleic acids. They are, however, of universal importance in living matter and significant developments throwing light on structure-function relations will surely emerge in the near future."
\end{abstract}

Gerald O. Aspinall (1970) ${ }^{1}$

However in the early 1980 's technology became available that greatly simplified the determination of oligosaccharide sequences ${ }^{2}$. This technological advance allowed the rapid characterisation of the carbohydrate portions of complex glycoproteins. Subsequent to this experiments were conducted to identify the importance of the carbohydrate to the function of a glycoprotein. A number of experiments then illustrated that the carbohydrate was central to the function of the glycoprotein ${ }^{3,4}$. Glycoconjugates have now been identified as being of primary importance to several significant biological processes ${ }^{5}$ such as mammalian fertilisation 6 , 7, viral replication $^{8-10}$, parasitic infection ${ }^{11}, 12$, cell growth ${ }^{13}$, cell-cell adhesion ${ }^{14}$ and immune response ${ }^{15}$. It is due to this biological significance that the study of carbohydrate chemistry is once again at the forefront of chemical research. 
Carbohydrates are commonly implicated in biological recognition processes. At their simplest level such processes involve the transfer of information through the recognition of one molecule by another. This molecular recognition is dependant on the 3-dimensional shape of both molecules. Therefore nature requires large repertoire of molecular shapes to differentiate between biological functions. Carbohydrates are important in nature because their versatility of structure and 3-dimensional shape means that very different shapes can be constructed from a small number of building blocks. The potential for diversity in oligosaccharides is greater than other biological polymers such as proteins and DNA/RNA because;

- There are a larger number of potential monomer building blocks (although only about ten monosaccharide units that are commonly found in mammalian glycoproteins and glycolipids ${ }^{16}$ (see Figure 1:3).

- The pattern of monomer connection has greater scope for variation (i.e. monomers may be connected between the 1 and the 4 positions or between the 1 and the 6 positions etc.).

- Branching can occur at any monomer unit.

- Each linkage between two monomers can occur in one of two possible configurations (either the $\alpha$ or $\beta$ anomer (see Figure 1:1)).

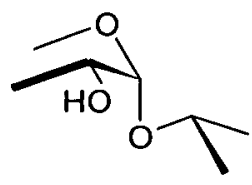

$\alpha$-Anomer

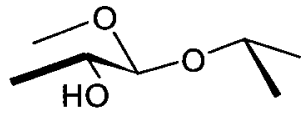

$\beta$-Anomer

Figure 1:1: $\alpha / \beta$ linkages.

This means that unlike proteins and DNA/RNA the sequence of monomer units does not adequately describe the structure of a complex carbohydrate. The description must include the pattern of the different linkages and their configuration (i.e. the abbreviated description of the carbohydrate in Figure 1:2 would be Galß1-4GlcNAc). 


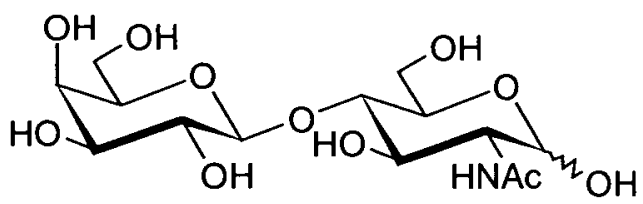

Figure 1:2: Galß1-4GlcNAc.

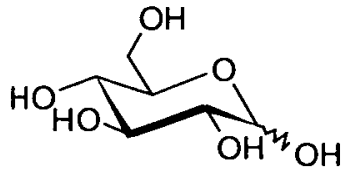

D-Glucose<smiles>OCC1O[C@H](O)[C@@H](O)[C@H](O)C1O</smiles>

D-Galactose

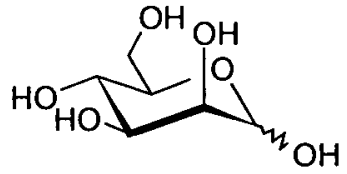

D-Mannose

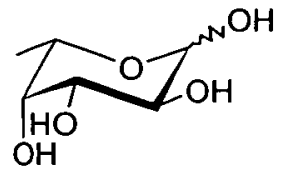

L-Fucose

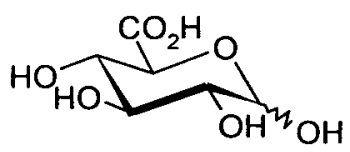

D-Glucuronic acid

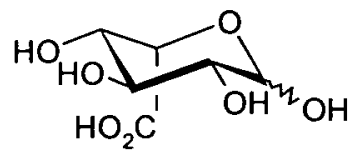

L-Iduronic acid

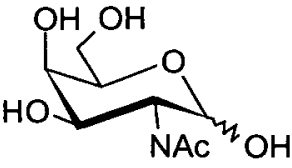

N-Acetyl galactosamine

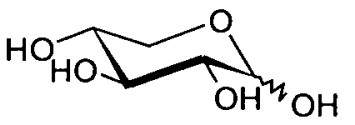

D-Xylose

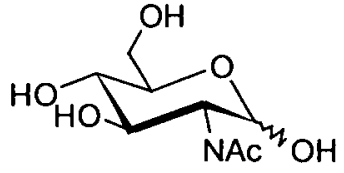

$\mathrm{N}$-Acetyl glucosamine

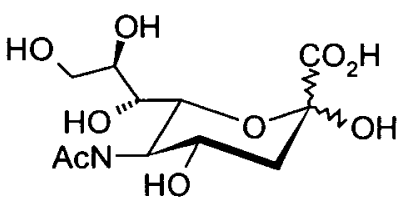

Sialic acid

Figure 1:3: Common monosaccharide units.

Carbohydrates on proteins are often composed of very similar repeating patterns of monosaccharide building blocks. However in situations where the carbohydrate is recognised by a carbohydrate binding protein, the carbohydrate structure often contains, on its periphery, unusual presentations of common sugar units or monosaccharide units that have further structural modifications ${ }^{17}$. It is these unusual structures and modifications that interact with the protein receptor and are responsible for efficient binding to the protein. The backbone structure of the polysaccharide may be considered as an elaborate scaffold used to present the binding functions in the correct fashion. 
There are many additional functional groups that are incorporated into carbohydrates which grant them unique properties. Commonly encountered structural additions include;

- Acetylation, of the hydroxyl group.

- Phosphorylation, of one or more hydroxyl groups.

- Oxidation of the hydroxyl groups to aldehydes/ketones or carboxylic acids.

The one trend that runs through all these additions is that all add a "handle" to the carbohydrate that influences the way in which they bind to a carbohydrate binding protein.

Most functional modifications are incorporated at a late stage in carbohydrate biosynthesis, added once the backbone structure has been constructed. This has led to the theory that sugar modification is a late evolutionary event, that carbohydrates are modified to adapt their role or to prevent their exploitation by infectious agents ${ }^{17}$. It would seem that nature is able to express other enzymes within the cell to modify carbohydrates when it requires a new structural feature.

The study of lectin proteins and the way in which they bind to carbohydrates has led to the discovery of a number of unusual functional additions. One such structural modification for carbohydrates is the incorporation of a sulfate ester (see Figure 1:4), referred to as sulfation.

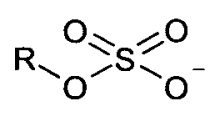

Figure 1:4: Sulfate monoester.

Sulfation appears to be an important alteration and the presence of a sulfate ester in a carbohydrate can grant a unique activity to an otherwise common structural motif ${ }^{18}$. 


\section{Sulfated Carbohydrates.}

The biological history of sulfated carbohydrates mirrors that of carbohydrates in general. Their presence in a variety of seaweeds was well documented during the first half of this century, as was their presence in mammalian tissue, particularly in the tissue of joints and other cartilaginous body parts ${ }^{19}$. However their importance was considered to be limited to the physical properties these compounds granted to the tissue in which they occurred. Most sulfated polysaccharides are excellent gelling agents, capable stratifying several times their dry mass of water and this was regarded as their key function in nature. However, latterly with the increased interest in biological carbohydrates and with changes in isolation and characterisation techniques a vast array of sulfated oligosaccharides have been found.

It would appear that sulfated carbohydrates are actually common throughout biological systems and have vital roles to play in a number of biological interactions. An in-depth discussion of their biosynthesis and biological action is beyond the scope of this thesis and this author however some of the more fascinating systems that involve sulfated compounds will be briefly examined. The following sections look at a number of examples of biologically important sulfated oligosaccharides. They show the diversity of natural systems that use sulfated compounds and highlight the importance of sulfation as a structural modification.

\subsection{Heparin}

No discussion of sulfated carbohydrates would be complete without some mention of Heparin. Heparin is a naturally occurring glycosaminoglycan (a polysaccharide which is made up of repeating disaccharide units consisting primarily of amino sugars) which is found attached to a number of proteins on mammalian cell surfaces. Heparin contains both sulfate monoesters $\left(-\mathrm{OSO}_{3}{ }^{-}\right)$and sulfamic amides $\left(-\mathrm{NHSO}_{3}{ }^{-}\right)$ at regular intervals throughout the polymer structure (see Figure 2:1). 
Although the name Heparin is derived from Hepatos the Greek word for the liver, the organ in which the compound was believed to be most abundant, the compound is actually commercially prepared from bovine lungs or porcine intestinal mucosa $\mathrm{a}^{20}$.

Heparin is probably best known because of its powerful anticoagulant properties. However in latter years the compound has been implicated in a host of other exciting - cell surface interactions.

\subsubsection{Anticoagulant properties of Heparin.}

The anticoagulant properties of Heparin were first discovered by Jay McLean, a medical student at the John Hopkins University in 1916 and it is still used to inhibit blood clotting to this day. Approximately 33 metric tons of heparin are produced annually representing around 500 million doses ${ }^{21}$. The compound is extremely fast acting and is primarily used to treat deep vein thrombosis and pulmonary embolism until oral drug administration can take effect.

The overall effect of Heparin addition is to inhibit Thrombin, an important protease enzyme. Thrombin converts fibrinogen to fibrin which is a vital step in blood clot formation. Heparin works as an anticoagulant by potentiating the effect of Antithrombin III (AT III) the natural inhibitor of Thrombin, indeed Heparin is thought to enhance the activity of AT III by 1,000 fold $^{22}$. Heparin binds to AT III in a 1:1 complex via a distinct pentasaccharide sequence (see Figure 2:1) that reoccurs frequently through the polymer.

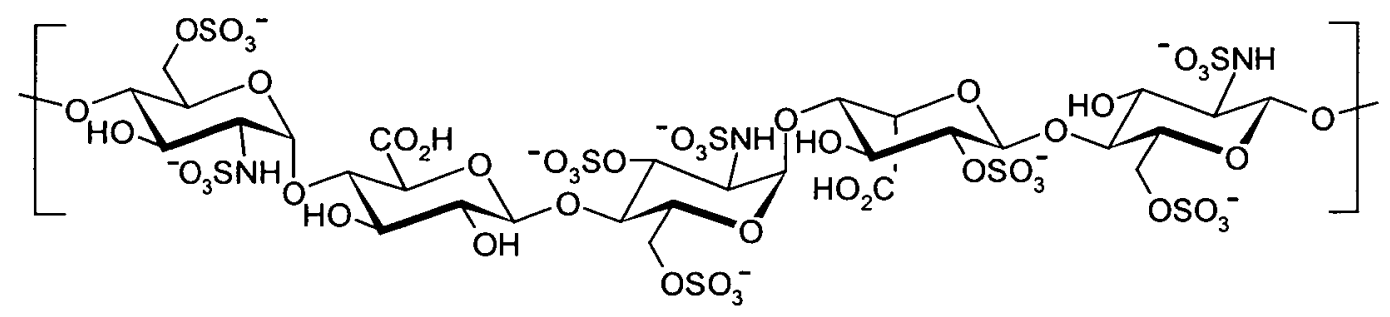

Figure 2:1: Antithrombin III binding sequence. 
Binding to Heparin initiates a conformational change in AT III which activates its antithrombitic activity ${ }^{23}$. Heparin also binds to Thrombin itself via a second remote binding site and serves to bring the two proteins into close proximity.

The Heparin/AT III complex also has the potential to bind to other proteins involved in the thrombosis cascade and it is known that it can inhibit clot formation through a number of other mechanisms 24 .

\subsubsection{Other roles of Heparin.}

In latter years Heparin has been found to bind to a host of other important proteins. Heparin and Heparan sulfate are constituent parts of a number of cell surface proteoglycans and participate in a number of cell surface/protein interactions.

Heparan sulfate side chains have been shown to bind to Fibroblast Growth Factor and a number of theories have been proposed to explain the biological significance of this binding. It has been proposed that the proteoglycans may act as a temporary storage area for the growth factor, granting it an immunity from roaming proteolytic enzymes that would otherwise break it down 25 . It has also been implied that the binding to the Heparan sulfate sidechains is a necessary part of the activation of the growth factor ${ }^{26}$. Fibroblast growth hormone has an important role in processes such as embryonic development, wound repair and tumour growth. Obviously these are areas of intense research because of the implications for a number of currently poorly treated disease states involving smooth muscle tissue growth such as tumour metastasis ${ }^{27}$ and the formation of scar tissue 28 . The interaction between heparin and fibroblast growth factor is a potential therapeutic target and this has stimulated efforts to identify and exploit the fibroblast binding sequence within the polymer $26,29-32$.

Although a molecule of intense study since its discovery the structure of Heparin is still not totally understood. It is only now that we are beginning to appreciate the full 
involvement of Heparin in cellular process and with reports of Heparin like molecules inhibiting the binding of HIV virus ${ }^{33,34}$ it is safe to say that the study of the biology of Heparin will continue for many years.

\subsection{Nod Factors}

Sulfated carbohydrates have a vital part to play in the recognition of certain beneficial bacteria by some leguminous plants.

Leguminous plants are unique in nature as they are able to survive on soils from which the nitrogen nutrients that all plants require have been depleted. They are able to survive because they colonise bacteria that convert nitrogen gas within the atmosphere into ammonia which the plant can use to produce the nitrogen containing molecules it requires. A number of Leguminous plants such as peas and clover colonise Rizobium bacteria in nodules on their roots and lower stems. Within the root nodules the plant feeds the bacteria with a rich food source and receives in return a source of nitrogen containing molecules. This symbiotic relationship places leguminous plants at a distinct advantage over other plants that obtain their nitrogen from compounds dissolved in the soil. Leguminous plants can thrive in areas where other plants cannot survive. This ability to grow on soil that has been depleted of its natural nutrients has led to the practice of planting leguminous plants such as clover on fallow fields to help rejuvenate overworked soil.

With the advent of molecular biology this important agricultural role has taken on a new dimension. If staple crops such as wheat, maize and rice can be genetically altered to nodulate and colonise Rizobium bacteria then the world demand for nitrogen fertilisers will drop significantly. This will reduce agriculture pollution and potentially help feed the world's burgeoning populations. For this reason a massive effort has been made to understand the symbiotic relationship between the plant and the bacteria. What has emerged from this research is a fascinating pathway of interspecies recognition and communication. 
Each Legume colonises a specific Rizobium strain to such an extent that a bacterium that normally nodulates a pea will have no effect at all upon an alfalfa plant however when an alfalfa plant comes into contact with a bacterium of the strain Rizobium Meliloti nodulation is initiated within a few hours. The key to this specificity lies in the chemical communications between the plant and the bacterium. In the case of Alfalfa and Rizobium Meliloti this has been found to involve a sulfated carbohydrate.

The process of nodulation is initiated by the alfalfa plant (see Figure 2:3). Amongst the many compounds released into soil by the plant are a number of flavanoid compounds. When these flavanoids come into contact with a Rizobium Meliloti bacterium they enter the cell and interact with a gene regulator protein. This interaction stimulates the transcription of a number of "nod" genes. These genes code for a series of highly specific "nodulation" proteins. These proteins have been identified, cloned and their function determined. The purpose of the nodulation proteins is to synthesis a Nodulation factor (Nod factor) which has been identified as a short chitin like tetra saccharide with lipid side chain and an acetate at the 6position of the nonreducing end and a sulfate monester at the 6-position of the reducing end (see Figure 2:2) 35 .

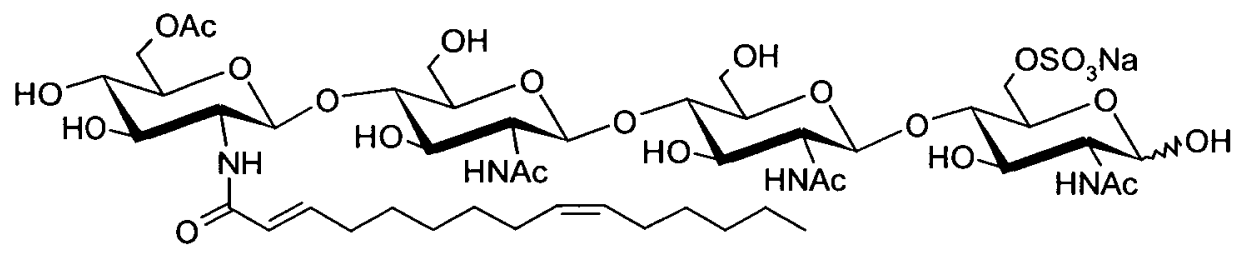

Figure 2:2: Alfalfa Nod Factor.

This compound is released from the cell and percolates its way back through the soil to the plant. On contact of the Nod factor with the root an amazing transformation begins to take place. Root hairs begin to extend and to encapsulate the bacterium. Cells within the root then begin to multiply and to reconfigure to build a nodule. Within nine days of first contact the nodule is complete and houses thousands of nitrogen fixing bacteria. 


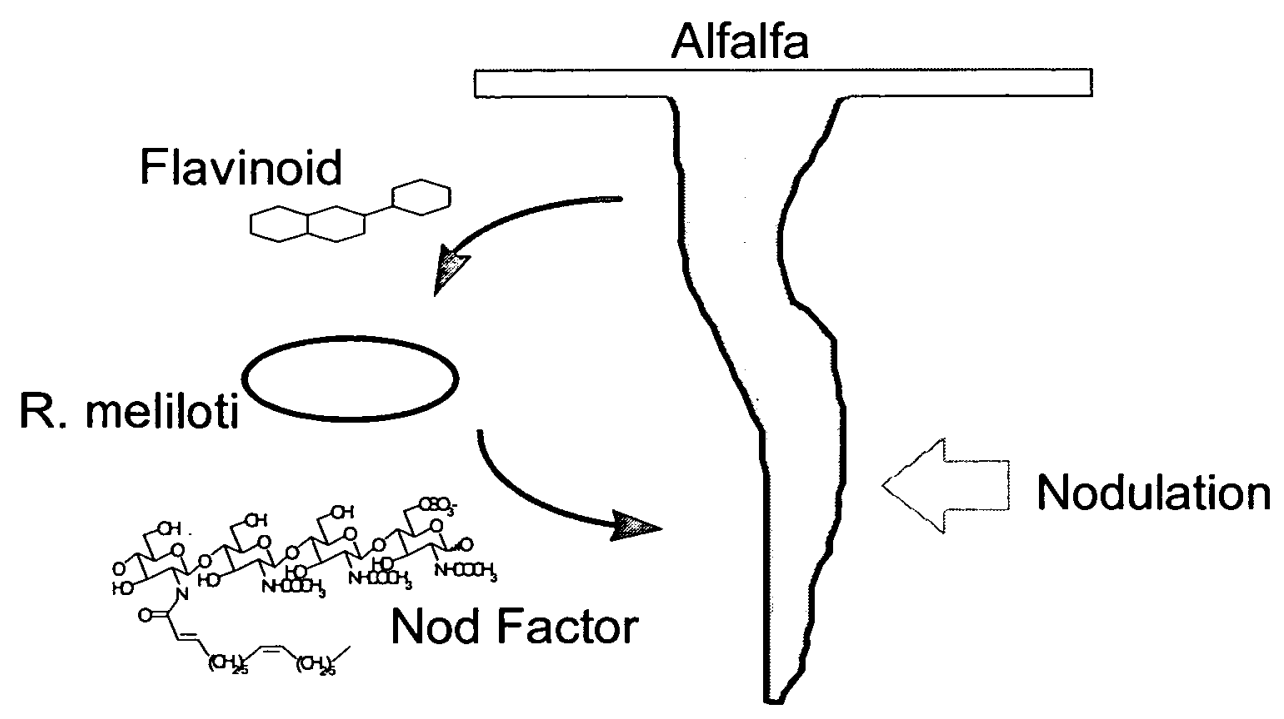

Figure 2:3: Nodulation process.

The Nod Factor is the key to the specificity of the plant for a particular strain of bacteria. The structure shown in Figure 2:2 is that of Alfalfa Nod Factor. Minute quantities of this compound will instigate nodulation in an Alfalfa plant even in the absence of the bacterium. The Pea Nod factor is almost identical but lacks the sulfate ester at the 6-position of the reducing end terminal sugar (see Figure 2:4). This compound has absolutely no effect upon alfalfa. This is a prime example of the importance of sulfate esters in granting unique functions to common sugar structures.

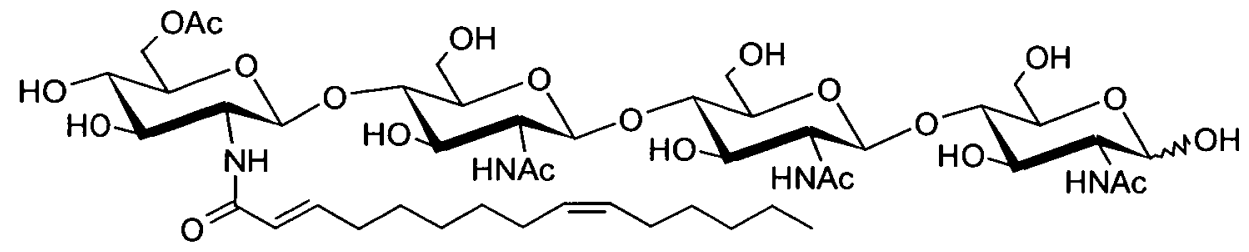

Figure 2:4: Pea Nod Factor.

The interaction between plant and bacterium is fascinating, as not only is it an example of two totally different species communicating but it is one of the first examples of a chitin type molecule having an effect on a plant. Previously plants were not thought to synthesise or respond to such compounds but in the light of this work other plants have been found to respond to Nod factor like molecules. This work has led to the discovery of a totally new and unexpected class of plant hormones 36 . 


\subsection{Sialyl Lewis Antigens}

This section details an area that has generated a huge amount of interest in the pharmaceutical industry within recent years. It is in part responsible for the current interest in carbohydrate chemistry and for the interest in sulfated carbohydrates specifically. This area is slightly different from the other examples given in this chapter as the sulfated compound in question is a synthetic mimic of the natural ligand that has been shown to have a greater affinity for the target protein.

The interest centres around a carbohydrate known as sialyl Lewis X (SLe ${ }^{\mathrm{x}}$ ) (see Figure 2:5), the proteins that bind to it, known collectively as Selectins and the role this interaction has in the process of leukocyte recruitment to a site of inflammation.

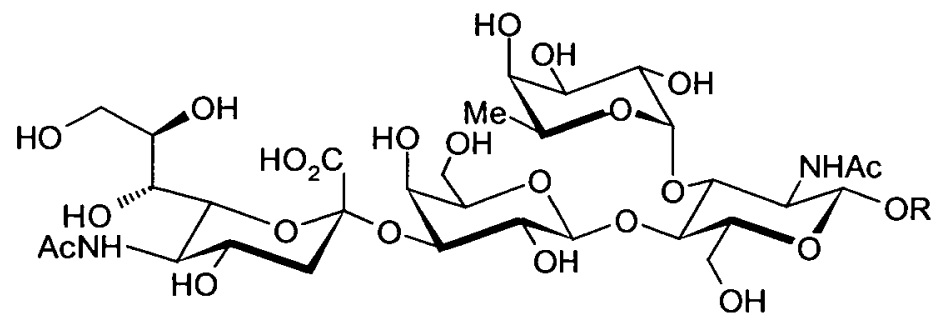

Figure 2:5: Sialyl Lewis X.

Sialyl Lewis $\mathrm{X}$ is found predominately on the surface of leukocytes and binds to Pselectin a cell surface protein expressed on endothelial cells in response to Thrombin during the inflammation process. This binding, although very weak in binding terms $\left(\mathrm{IC}_{50}\right.$ values of 1-3nM $\left.\mathrm{n}^{37}\right)$, slows the passage of the leukocyte in the blood stream and induces a rolling motion. As it rolls the leukocyte is slowed further allowing much stronger protein-protein interactions to take over. The leukocytes then becomes adhered to the blood vessel wall before extricating itself into the surrounding damaged tissue (see Figure 2:6). A compound that can interfere with this selectincarbohydrate interaction could reduce leukocyte recruitment and therefore be used to treat inflammatory diseases such as arthritis. 


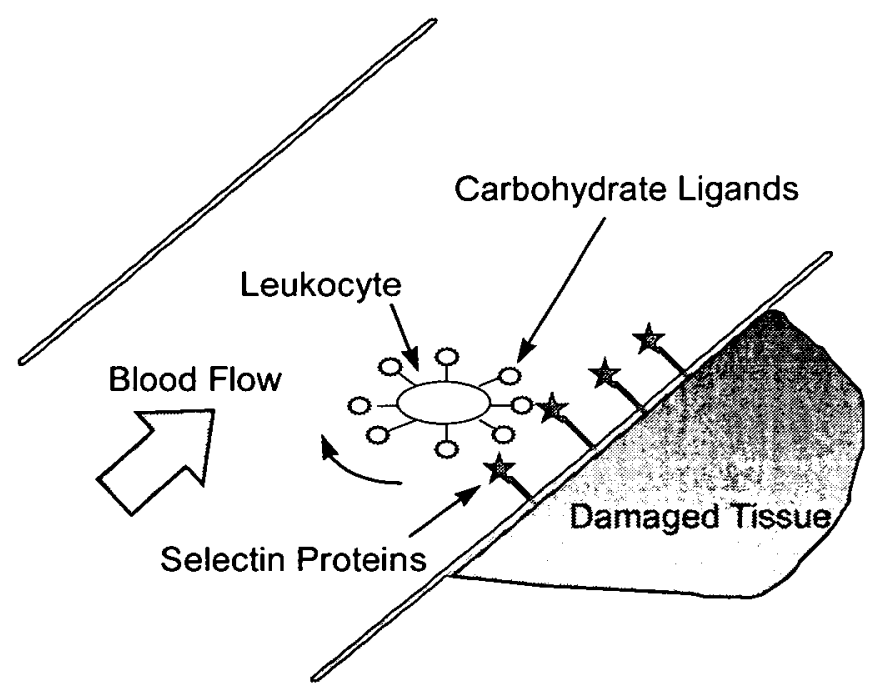

Figure 2:6: Leukocyte recruitment ${ }^{38}$.

The significance to sulfated carbohydrates comes from a report by Feizi 39,40 and coworkers that Selectins have a higher binding affinity for a sulfated version of the Lewis antigen (see Figure 2:7) than the natural compound thus making the sulfated carbohydrates very interesting to the pharmaceutical industry who are seeking new treatments for inflammation related disease states.

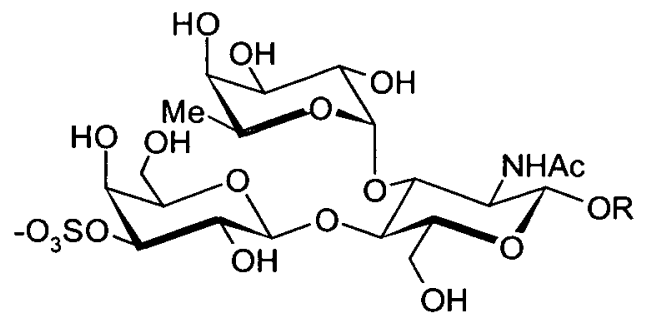

Figure 2:7: 3-sulfated Lewis $X$ antigen.

The Lewis X-selectin interaction may also be important in the process of cancer metastasis 41,42 adding to the importance of inhibitor studies.

The phenomena of Lewis $\mathrm{X}$ binding to Selectin proteins and its potential as a therapeutic target has provoked an enormous amount of effort by the pharmaceutical industry to produce carbohydrate like compounds that can act as inhibitors to the lectin carbohydrate binding. 
Another recent development related to this area is in the work of Kiessling and coworkers who have synthesised a short length polymer containing sulfated Lewis X type carbohydrates (see Figure 2:8). Not only does this soluble polymer have a far higher binding affinity than the monomeric structures ${ }^{43}$ it can also induce L-selectin protein shedding from the cell surface ${ }^{44}$. It has been proposed that shedding is induced by clustering of the proteins by a large multivalent molecule such as the glycoproteins expressing Lewis $\mathrm{X}$ on the leukocyte cell surface.

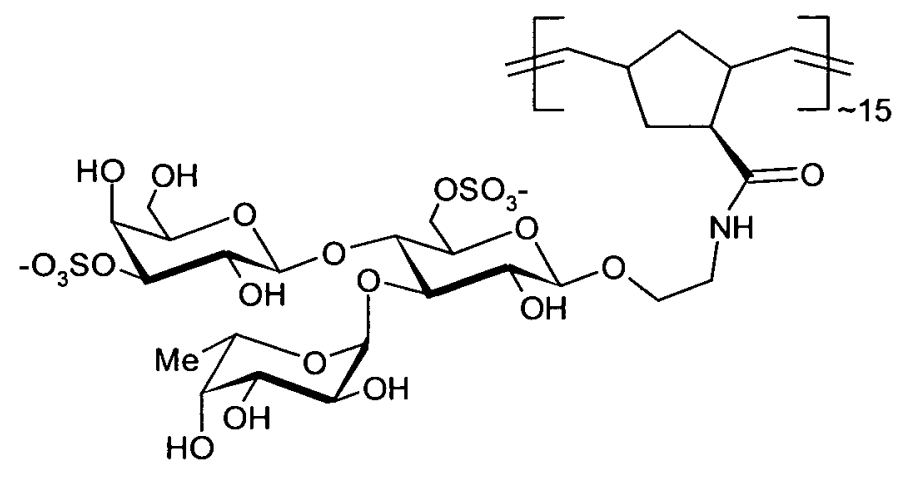

Figure 2:8: 3',6-disulfo Le ${ }^{x}$ neoglycopolymer.

Protein shedding is the process by which a cell surface protein is removed from the cell surface by localised protease enzymes ${ }^{45}$. It is an important mechanism by which the biological system can down regulate a ligand/receptor interaction by simply cutting away the receptor. This shedding process is also an important source of soluble proteins that may act as local or distant hormones.

It is interesting to note that many of the proteins that have been found to be shed from their cellular surface are involved in systems $\mathrm{s}^{45}$ in which a sulfated carbohydrate ligand has been implicated ${ }^{46}$. Although this may only be coincidence.

The previous sections have examined some interesting examples of biologically important carbohydrates and have given some justification as to why such compounds are synthetic targets. However sulfated compounds are relatively common in biological systems and any advances in the synthesis of sulfated carbohydrates will have repercussions on the synthesis of other sulfated compounds. 
The following section briefly considers a number of notable non carbohydrate sulfated compounds.

\section{Non Carbohydrate Sulfates.}

Sulfation is common throughout nature and many non carbohydrate derived compounds have been characterised that contain a sulfate ester. This is partly because sulfation greatly increases the water solubility of organic molecules. Organs such as the liver are full of enzymes capable of sulfating toxins as a method for accelerating their removal by urinary excretion.

This section briefly mentions some other sulfated natural products.

\subsection{Sulfated Proteins.}

Sulfation is believed to be the most common post translational modification of tyrosine residues 47 and sulfated proteins are widespread throughout almost every species examined ${ }^{48}$. The exact function of the sulfated residues is not known in most cases but it is safe to assume that such a hydrophilic and acidic residue will have a pronounced effect on the biological and/or physical properties of the protein of the protein.

One example is that of P-selectin glycoprotein ligand-1 (PSGL-1). As discussed in the previous section, P-Selectin is a lectin type protein expressed on endothelial cell surfaces during the cellular response to tissue damage. P-Selectin binds to carbohydrate ligands on the surface of passing leukocytes. The protein on the leukocyte that presents the carbohydrate ligands is P-selectin glycoprotein ligand-1 (PSGL-1). It has been found that the protein backbone of PSGL-1 must be sulfated at a tyrosine residue to induce the high binding affinities required for adhesion of the leukocyte to the endothelium 49 . 
Protein sulfation is a poorly examined subject. Although a number of naturally occurring sulfated proteins are known there are very few published attempts at the synthesis of such compounds. Those that have examined this synthetic challenge construct the protein backbone first then sulfate randomly and rely on extensive purification techniques to isolate the correct product. Any successes in the field of sulfate protection would have definite applications in the area of sulfated protein synthesis.

\subsection{Sulfated Marine Natural Products.}

Sulfur is the fourth most important element in sea water after chlorine, sodium and magnesium and the sulfate ion is by far the most abundant form of sulfur. It is therefore no great surprise that sulfated compounds are common in marine organisms. Most of the early work done on sulfated carbohydrates focused of compounds extracted from various seaweeds ${ }^{19}$ but there are also a host of other interesting sulfated molecules that have now been isolated.<smiles>CC(C)C(=O)O[C@H](C)[C@@H](NC(=O)C(O)COS(=O)(=O)O)C(=O)NC(CCCNC(=N)N)C(=O)NC1CCC(C)C(C(Cc2ccccc2)C(=O)N(C)C(Cc2ccc(O)cc2)C(=O)NC(C(=O)O)C(C)C)C1=O</smiles>

Figure 3:1: Micropeptin 9051.

A recent review lists over 500 sulfated compounds (not including macromolecules) that have been isolated from marine sources ${ }^{50}$. Figure 3:1, Figure 3:2 and Figure 3:3 
show some examples of the fascinating and diverse structures that have been characterised.<smiles>CC1=C(Cc2cc(CO)cc(O)c2OS(=O)(=O)O)[C@@H]2CCCC(C)(C)C2CC1</smiles>

Figure 3:2: Siphonodictyol ${ }^{52}$.<smiles>C[As](C)(=O)C[C@H]1O[C@H](CCC(O)CO[Na])[C@@H](O)[C@H]1O</smiles>

Figure 3:3: 5-Deoxy-5-dimethylarsinoyl- $\beta$-D-ribofuranosyl-2-hydroxypropyl sulfate $^{53}$.

Maitotoxin, a polyether isolated from the dinoflagellate Gambierdiscus toxicus is considered to be the most complex non polymeric natural product ever isolated ${ }^{54,55}$ (see Figure 3:4).

As the biological screening of such amazing compounds continues and interesting biological properties are discovered there will be a demand for synthetic routes to these sulfated compounds. Any methods developed for the synthesis of sulfated carbohydrates should have direct application within this small area of total synthesis. 


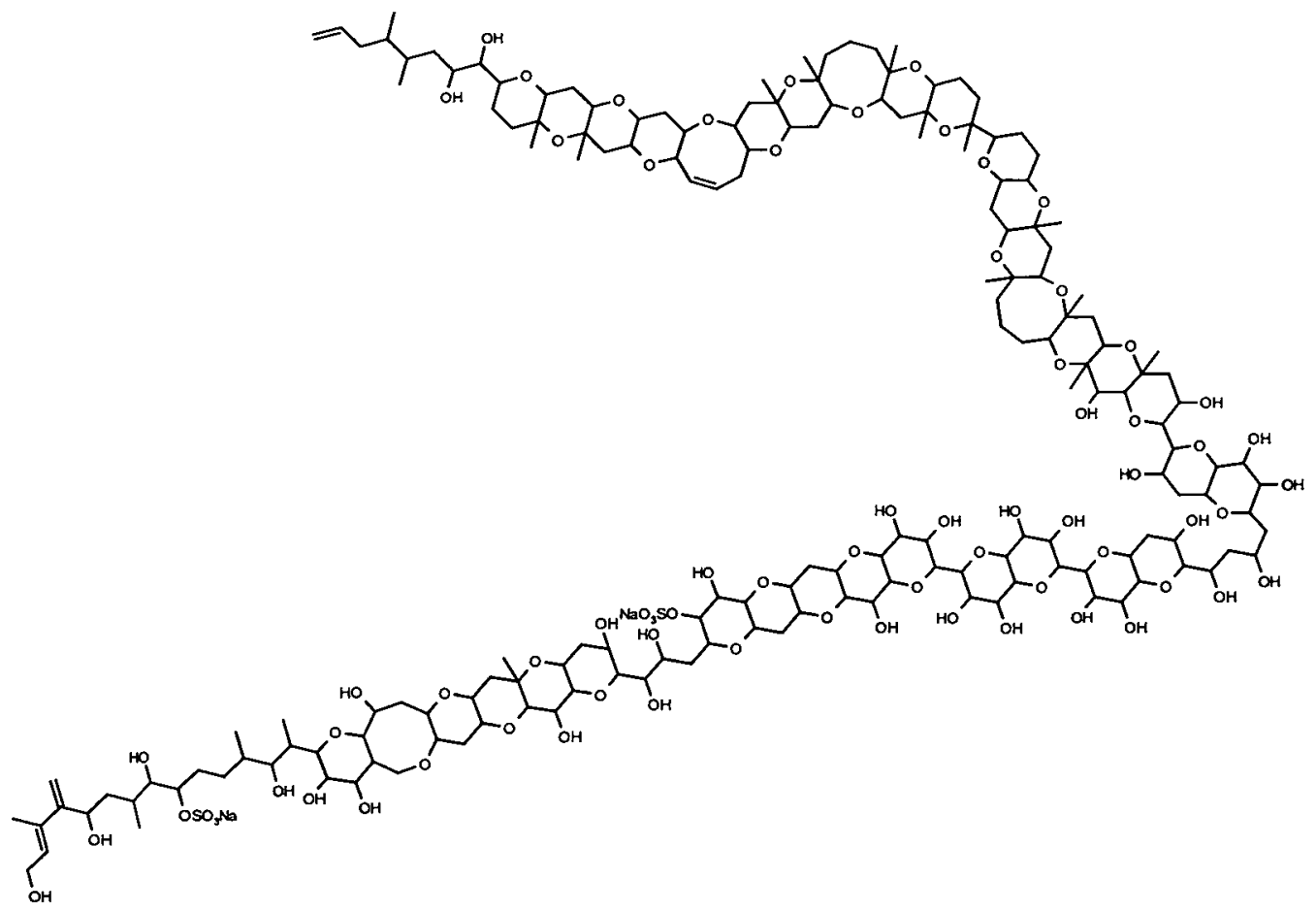

Figure 3:4: Maitotoxin.

\section{Synthesis of Sulfated Carbohydrates.}

This section examines the various methods that maybe employed to introduce a sulfate ester into a carbohydrate molecule. The second section examines in detail various synthetic methods for the transformation but it is prudent to first consider how nature produces sulfated carbohydrates.

\subsection{Sulfotransferase Enzymes.}

Sulfotransferases are a large family of proteins that transfer a sulfate group to numerous endogenous and exogenous chemicals. All rely on a single cofactor, 3'phospho-adenosine 5'phosphosulfate (PAPS) (see Figure 4:1) as their source of sulfate 56 . 
<smiles>Nc1ncnc2c1ncn2C1OC(COP(=O)([O-])OS(=O)(=O)[O-])C(OP(=O)([O-])[O-])C1O</smiles>

\section{Figure 4:1: 3'-Phospho-adenosine 5'phosphosulfate}

Little is known about the mechanism of sulfotransferase catalysed sulfation and there is very little sequence homology between different sulfotransferases proteins isolated so far.

There is enormous variety in the compounds that can be sulfated by sulfotransferase enzymes. Two distinct families of sulfotransferases have been identified and characterised. Cytosolic sulfotransferases are generally responsible for the sulfation of non-natural compounds such as drugs ${ }^{57}$ and toxins ${ }^{58}$ as well as many endogenous substances 59 . Membrane bound sulfotransferases are mainly confined to the Golgi apparatus and produce the majority of the sulfated glycosaminoglycans, glycoproteins and proteins that are secreted by a cell. While cytosolic sulfotransferases have a broad substrate tolerance, membrane bound sulfotransferases, which contain the group of carbohydrate specific sulfotransferases, are highly specific both in terms of their substrate and the point of sulfation.

Carbohydrate sulfotransferase enzymes selectively sulfate at a single point in a complex structure and they fall into two broad categories, those for which the primary recognition point is the carbohydrate itself 60 and those that recognise the protein backbone but sulfate the attached carbohydrate 61 . These enzymes have been found to be highly specific for their natural substrates and there has been little success in enzymatic sulfation of unnatural substrates using these enzymes. An added complication to the development of a general enzymatic sulfation protocol is the reliance on PAPS as an essential cofactor. PAPS is highly unstable and extremely 
expensive to purchase. The only successful synthetic application of a sulfotransferase enzyme was published by Wong and co-workers who had cleverly incorporated a PAPS synthase enzyme into their enzymatic system ${ }^{62}$. Despite this success there seems little prospect of the regular use of PAPS dependant sulfotransferase enzymes for synthetic applications.

As the understanding of the role of sulfated carbohydrates increases it seems likely that they will become important therapeutic targets. Compounds that can act as inhibitors of sulfotransferase enzymes may be then be viable therapeutic objectives. Currently the only known sulfotransferase inhibitors are for aryl sulfotransferase enzymes. It is possible that any 'protected' sulfated oligosaccharide might act as a highly selective inhibitor of carbohydrate sulfotransferase as they are both "substrate" and "product" like.

The deliberate introduction of a sulfate ester into a carbohydrate as part of a synthetic program is one transformation that is more easily accomplished using chemical methods than enzymaticaly. A number of methods are described within the literature. The following section briefly examines a number of the known procedures and discusses their utility in carbohydrate synthesis.

\subsection{Chemical Methods.}

There exist a number of methods to introduce a sulfate monoester into a molecule. This section reviews some of the reagents used to perform a sulfation and considers some of the adaptations that can be made to enhance the utility of these methods.

\subsubsection{Chlorosulfonic acid.}

The use of chlorosulfonic acid in pyridine to sulfate a free hydroxyl group (see Figure $4: 2$ ) is one of the early methods used to produce sulfate monoesters and is no longer commonly used due to the inhospitable reaction conditions and the difficulty experienced in isolating the product from the reaction mixture. 
<smiles>[R]OOS(=O)(=O)O[R]</smiles>

Figure 4:2: Chlorosulfonic acid.

The mechanism of the reaction is not clear. It may occur either by an $\mathrm{S}_{\mathrm{N}} 2$ process in which the chloride of the chlorosulfonic acid is displaced by the alkoxide of the alcohol or by a $S_{N} 1$ process in which hydrogen chloride is eliminated from the chlorosulfonic acid with the in situ formation of sulfur trioxide which is then attacked by the alkoxide. What ever the mechanism, the reaction conditions are highly acidic, certainly it cannot be used in the presence of $\mathrm{H}^{+}$sensitive protecting groups and in extreme cases it may cause acidic hydrolysis of the glycosidic linkages. E. G. V. Percival reported the sulfation of diacetone glucose with chlorosulfonic acid 63 . The low yield of the reaction is due to the hydrolysis of the isopropylidene acetal groups during the course of the reaction however the author makes no mention of isolating any by-products (see Figure 4:3).

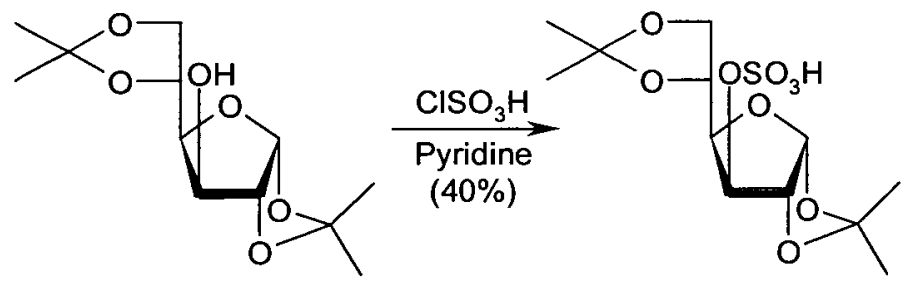

Figure 4:3: Sulfation with chlorosulfonic acid.

\subsubsection{Bisulfite.}

This is another old technique that has no real general applicability. This method is only noteworthy because it is one of the few examples of a selective sulfation of an unprotected sugar structure. 6-Sulfo-D-glucose is the product of the reaction of glucose with bisulfite at $100^{\circ} \mathrm{C}^{64}$ (see Figure 4:4).

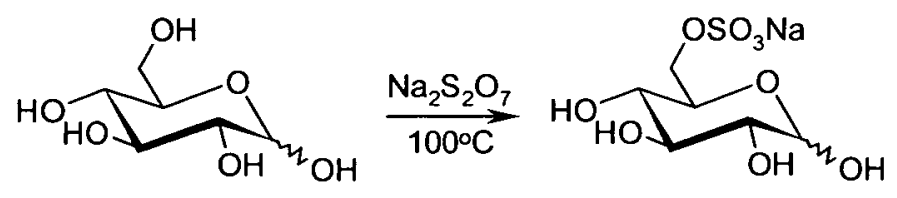

Figure 4:4: Sulfation by bisulfite. 
Although the reaction conditions are too extreme for general use it could be considered as a source of 6-sulfo sugars as the reaction appears to be general for aldose sugars.

\subsubsection{Sulfuric acid with coupling reagents.}

The use of sulfuric acid in conjunction with a "coupling" reagent such as $N, N$ 'dicyclohexylcarbodiimide (DCC) is an interesting method of introducing sulfate monoesters that has received little application since it was first published in the late 1960 's ${ }^{65-67}$. The method was primarily aimed at the rapid synthesis of ${ }^{35} \mathrm{~S}$ containing carbohydrate sulfate monoesters as ${ }^{35} \mathrm{SO}_{4}{ }^{2-}$ is readily available. This reaction is interesting as it is an example of $\mathrm{SO}_{4}{ }^{2-}$ acting as a nucleophile. Nucleophilicity is not a property normally associated with such a stable anion.

Addition of sulfuric acid to a mixture of acetal protected carbohydrates and DCC in DMF was used to synthesis a number of compounds (see Figure 4:5) although the authors do note that the acetals are occasionally hydrolysed 65 .

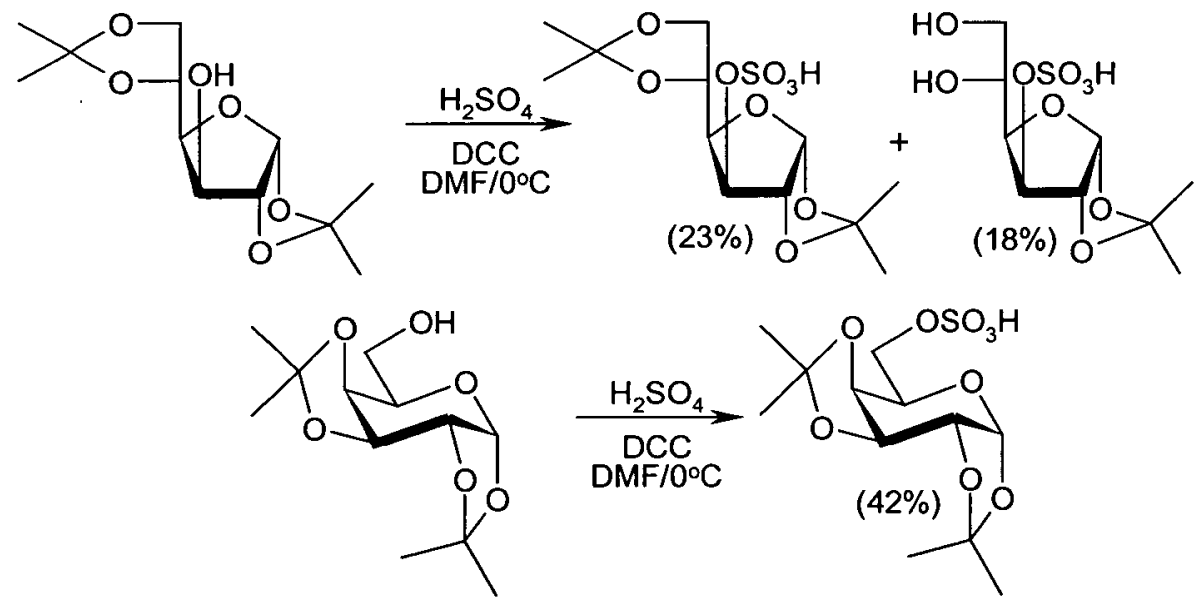

Figure 4:5: Sulfuric acid coupling.

\subsubsection{Oxidation of sulfites.}

This again is an older method not used predominately today. The method has been used more for the synthesis of cyclic sulfates than for sulfate monoesters. It is 
difficult to introduce a cyclic sulfate into a diol directly using sulfonyl chloride because the intermediate chlorosulfate is too easily displaced in an $\mathrm{S}_{\mathrm{N}} 2$ fashion by the liberated chloride (see Figure 4:6).

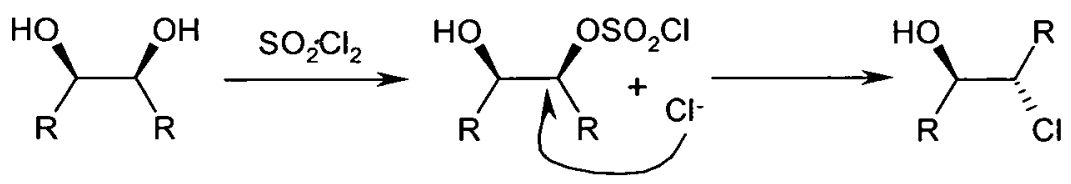

Figure 4:6: Sulfonyl chloride reaction.

To avoid this the sulfur centre can be introduced at a lower oxidation level (sulfite) by reacting the diol with thionyl chloride 68 (the second chloride is much more reactive in thionyl chloride due to the differing electronic structure of the molecule) the cyclic sulfite produced may then be oxidised to the sulfate. The most commonly used oxidants are aqueous solutions of potassium (or sodium) permanganate ${ }^{69}$. An adaptation by Tewson and co-workers ${ }^{70}$ allows the oxidation to be performed in a biphasic system and avoids alkaline hydrolysis of the product. Another method for the oxidation of cyclic sulfites has been pioneered by Sharpless and co-workers who have modified their ruthenium oxide catalyst system to perform the reaction ${ }^{71}$.

Non cyclic sulfite diesters may also be oxidised to the corresponding sulfate ${ }^{72}$ using calcium permanganate or even activated manganese dioxide ${ }^{73}$. Although these methods are successful their utility in a synthesis is extremely limited as they can only be employed upon molecules that do not have any other oxidizable functionality. Also these routes involve multiple steps and often require intermediate purification.

\subsubsection{Complexes of sulfur trioxide and amines.}

This is by far the most widely used and simplest method of sulfating a free hydroxyl group ${ }^{\#}$. Sulfur trioxide gas readily forms crystalline solids with a variety of Lewis bases such as triethylamine, trimethylamine and $N, N$-dimethyl formamide pyridine

\footnotetext{
\# For example all of the six syntheses of sulfated Alfalfa Nod factor published from 1984 to 1998 employed a sulfur trioxide/amine complex as the sulfating agent ${ }^{74-79}$.
} 
(all of which are commercially available). These complexes can be used as easily handled sources of sulfur trioxide which is a powerful electrophile ${ }^{80}$. Reactions are normally carried out using $N, N$-dimethyl formamide as solvent although 1,4-dioxane and acetonitrile can be used. The reaction proceeds by a direct nucleophilic attack by the alkoxide of the alcohol onto the electrophilic sulfur atom of the sulfur trioxide. This preparative route is probably the mildest method for introducing sulfate esters although the solutions of sulfur trioxide and amine are highly acidic if permitted to come into contact with traces of water.

It has also been claimed that complexes of sulfur trioxide and tertiary amines can be used to form sulfate monoesters at the anomeric position of the sugar ring. Such compounds have been used as glycosyl donors ${ }^{81}$ see Figure 4:7.

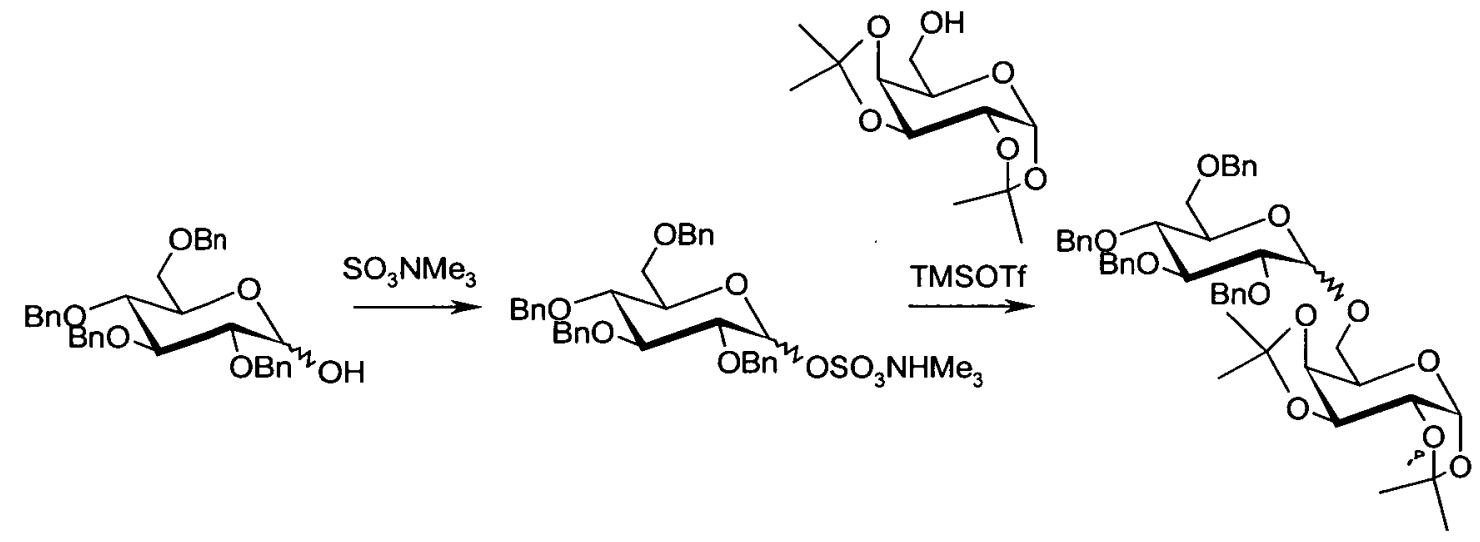

Figure 4:7: Sulfates as glycosyl donors.

\subsection{Selective Sulfation.}

All the previously mentioned sulfation methods are highly efficient methods of introducing a sulfate monoester into a carbohydrate so much so that hydroxyl group protection must be employed to prevent multiple and undesired sulfation taking place. Uncontrolled sulfation of methyl 4,6-O-benzylidene- $\alpha$-D-glucopyranose leads to a mixture of the two possible monosulfated compound and the disulfated compound (see Figure 4:8). 


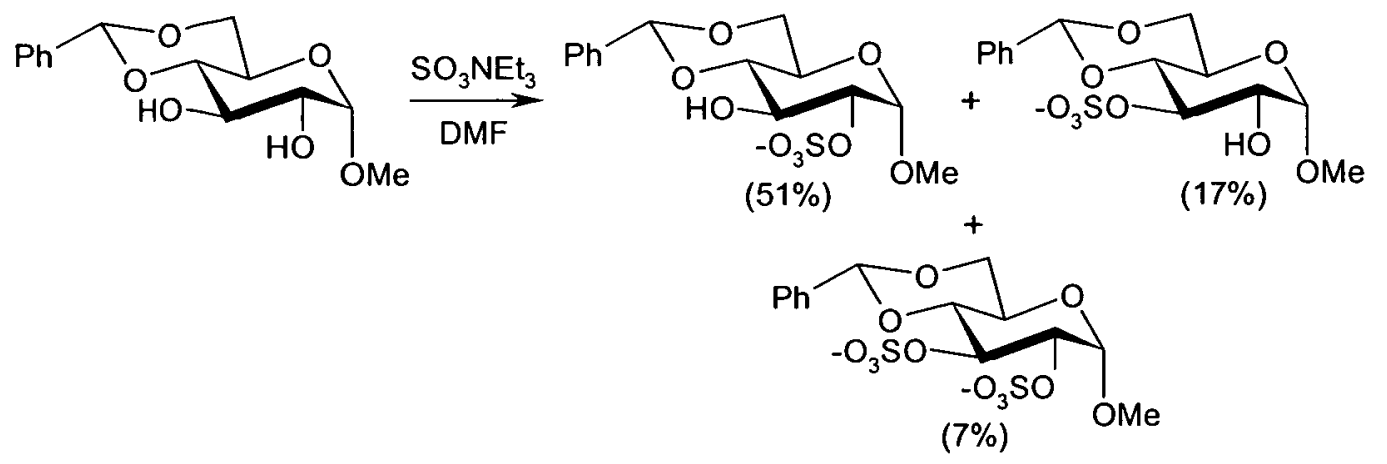

Figure 4:8: Uncontrolled sulfation.

By controlling the number of equivalents of sulfating agent it is possible to control the quantity of sulfate esters introduced however it is usually difficult to control the regioselectivity of sulfation unless a primary sulfate is desired ${ }^{82}$. For instance sulfation of $p$-methoxybenzyl 6-O-tert-butyldimethylsilyl- $\beta$-D-glucopyranoside with 1.5eq of $\mathrm{SO}_{3}-\mathrm{NMe}_{3}$ complex in pyridine gives a mixture of the 3-O-sulfate (68\%) and the 2-O-sulfate (21\%) (see Figure 4:9).
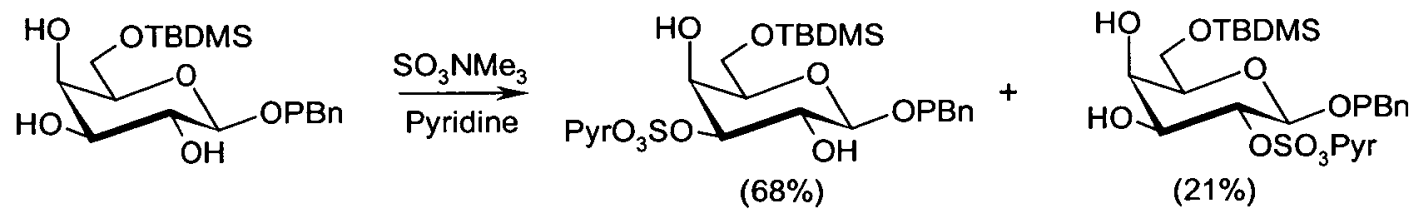

Figure 4:9: Direct sulfation.

One method for introducing selectivity into carbohydrate chemistry that is now well established is to exploit the chemistry of tin acetals. Dibutyltin oxide is known to react with diols to form five membered (and occasionally six and seven membered) acetals. The acetal formation is preferred when the diol has a cis configuration 83,84 . In such complexes there is considerable enhancement of the nucleophilicity of the equatorial hydroxyl group 85 . The exact explanation for this is not yet clear but what is clear from $\mathrm{nmr}$ and $\mathrm{X}$-ray diffraction studies $86-88$ of these compounds is that they do not exit as monomeric units but rather as dimers or higher oligomers. There is still some discussion as to how these structures affect the nucleophilicity of the hydroxyl groups. However it is possible to exploit both these regioselectivities to introduce an electrophile into a predictable point in an unprotected carbohydrate. This work was 
initial aimed at the regioselective introduction of acyl, alkyl, tosyl, and silyl groups $^{84,} 89,90$ but recently workers within the Flitsch group 91,92 and others 93,94 have extended this to sulfur trioxide as an electrophile. Using this methodology a sulfate may be introduced into a predetermined position in an unprotected carbohydrate (see Figure 4:10 and Figure 4:11).
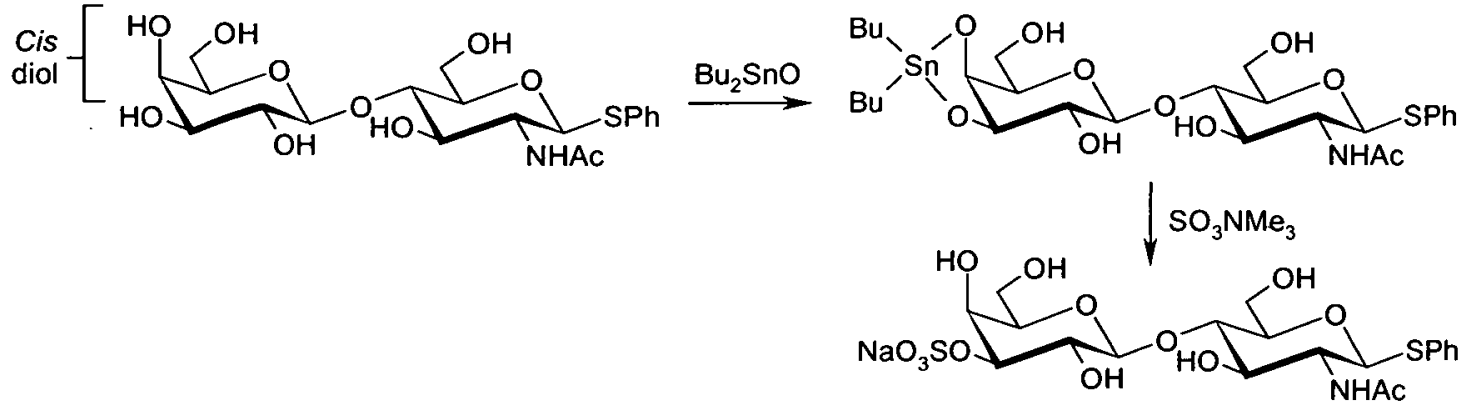

(83\%)

Figure 4:10: Regioselective sulfation of a thio maltoside.
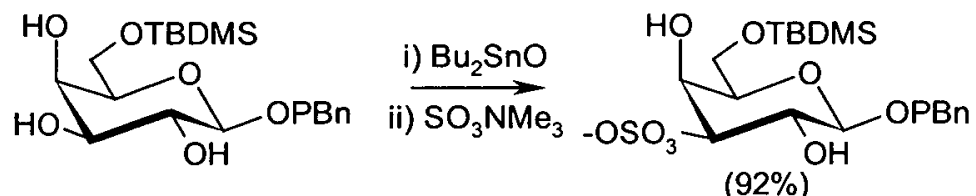

Figure 4:11: Regioselective sulfation of a galactose derivative.

The advantages of the tin acetal chemistry can be used on molecules that have no cis diols however the dibutyl stannylene acetal formation is not as facile and usually requires prolonged heating to adequately form the tin complex. Again treatment of such compounds with complexes of sulfur trioxide leads to the regioselective introduction of a sulfate ester (see Figure 4:12).
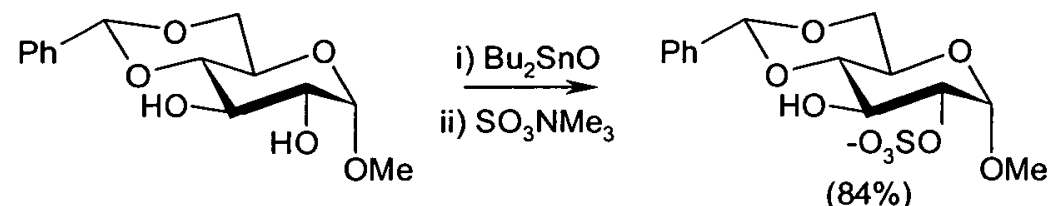

Figure 4:12: Selective sulfation.

The use of the tin acetal chemistry is an important method for regioselective sulfation and greatly simplifies the synthesis of some carbohydrates. 
Although it is possible to selectively introduce a sulfate into some carbohydrates using either the tin acetal chemistry or by regioselective sulfation of a primary hydroxyl group out side these special examples there exists no general procedure to selectively sulfate a chosen hydroxyl group.

\subsection{The Problem with Synthesis.}

As has been discussed there are a host of methods that can be used to introduce a sulfate ester into a carbohydrate; all are relatively aggressive and with a few special exceptions cannot be controlled to give good regioselectivities. Thus if a sulfated carbohydrate is to be synthesised a careful protection strategy must be adopted. Not only must the point of sulfation be picked out with an orthogonal protecting group, the other positions must be globally protected with acid stable protecting groups to prevent removal during the acidic sulfation procedure. Such protecting strategies greatly complicate a synthesis. A further complication is that all the sulfation techniques described so far yield the sulfate ester in its ionic form. Sulfate monoesters are highly acidic, as is expected of an ester of sulfuric acid (Heparin is possibly the strongest naturally occurring organic acid). Indeed sulfates are such strong acids, that in their protonated form they are capable of causing acid cleavage of glycosidic linkages within the molecules of which they are constituents. As a result of this sulfates are almost invariable handled in their salt forms, either as a conjugate with the amine used in the sulfation method or through the use of ion exchange resins the sodium or potassium salt form. Many early papers cite the conversion of the sulfate to its barium salt ${ }^{95}$ form as a useful preparative procedure; however this technique has fallen out of favour as the insolubility of the salt renders the product impossible to use in subsequent steps. Although the salts of sulfates are often stable they are by no means easily handled. Even the salts of sodium and potassium have limited solubility in organic solvents. Their high polarity complicates chromatography and the sulfate esters are known to interfere with other chemical transformations 96 . 
The net result of these properties is that sulfation is, almost without exception, one of the last steps performed in a synthesis. The point of sulfation is normally determined at the outset, protected with an orthogonal protecting group and carried through the synthesis. The protecting group is then removed and the hydroxyl group sulfated. The only steps attempted subsequent to the sulfation step are those required to remove the global protection. A good example of this strategy is found in Nicolaou and co-workers synthesis of the aforementioned Alfalfa Nod factor ${ }^{74}$ (see Figure $4: 13)$.

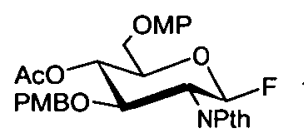

(a)

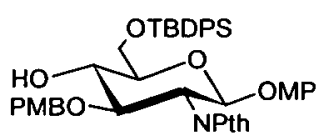

(b)

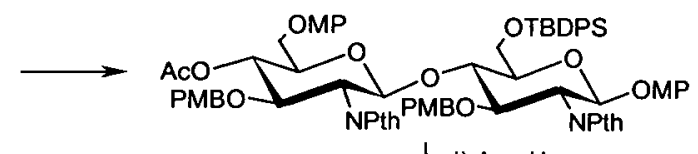

i) $\mathrm{AC}-\mathrm{H}$
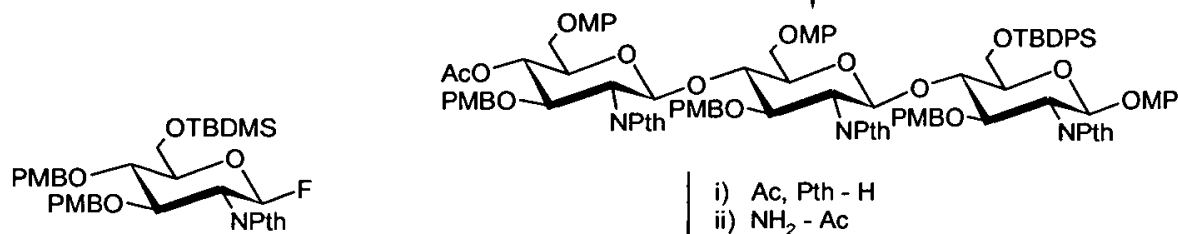

(c)

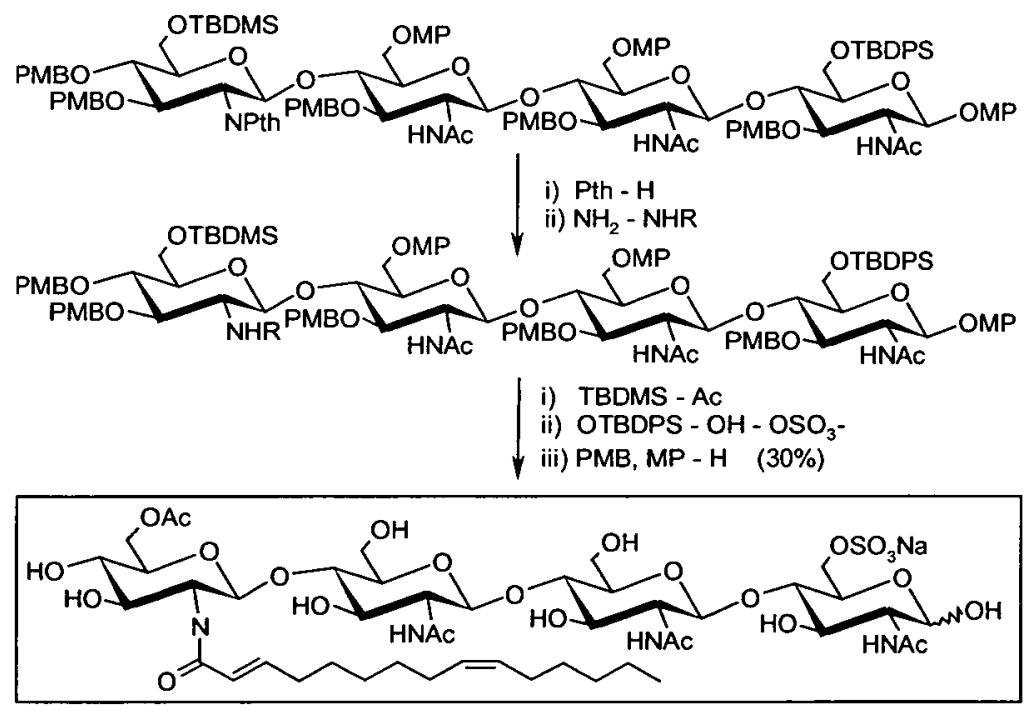

Figure 4:13: Synthesis of NodRm-IV.

The hydroxyl group is selected for subsequent sulfation by its initial protection with a tert-butyl diisopropyl silyl (TBDPS) protecting group. It is carried through the lengthy synthesis only to be unmasked at the terminus of the synthesis and sulfated using a complex of sulfur trioxide and trimethyl amine. The only synthetic 
transformation attempted post sulfation is the removal of the p-methoxybenzyl protecting groups that have guarded the other hydroxyl groups. The yield for this step is surprisingly low even considering that there are eight protecting groups being removed in one step. This can be attributed to the problems inherent in manipulating sulfated compounds.

The synthesis of complex molecules containing sulfate monoesters would be greatly simplified if the ionic nature of the sulfate could be masked in some way, by a protecting group.

\section{Sulfate Protecting Groups.}

A synthesis utilising a sulfate ester protecting group would have significant advantages over current synthetic approaches. The sulfate could be introduced at any convenient point in the synthesis and carried through in its protected form. The sulfate could then be unleashed as one of the final acts in the synthesis. A suitable sulfate protecting group would allow sulfate esters to be introduced into simple monomer building blocks for incorporation into more complex structures. Given the current interest in combinatorial chemistry and the drive towards solid phase carbohydrate synthesis this is an important potential application.

\subsection{Requirements of a Sulfate Protecting Group.}

This section explores the properties a protecting group for sulfates would need, to be of use in synthesis.

The requirements for a sulfate protecting group are in general no different from the requirements of any protecting group. The protection/incorporation step must be high yielding and reliable as must the deprotection/removal step. The protected functionality must be stable to the reaction conditions used to manipulate the rest of the molecule. However a sulfate monoester protecting group does not need to be truly orthogonal. It should be stable to the conditions used to manipulate other common protecting groups since this is going to be a central part of any carbohydrate synthesis but it does not need to be removable in the presence of other protecting 
groups. Due to the difficulties associated with handling sulfate monoesters the aim will be to remove the protecting group from the sulfate as the terminal step in the synthesis once the other protecting groups have been stripped away. Therefore the sulfate deprotection protocol need only be compatible with the target product itself.

\subsection{Development of a Sulfate Protecting Group.}

This section discusses how the approach to a protecting group evolved and how a protecting group with the desired properties was targeted. This section also describes how the objectives for the project were set and the problems that were foreseen.

\subsubsection{Sulfate diesters.}

The only obvious way to mask the anionic nature of the sulfate monoester is to convert it to a diester (see Figure 5:1).

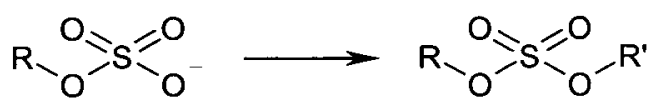

Figure 5:1: Monoester to diester.

The next sections discuss the properties of sulfate diesters in general and how these properties would affect a potential protecting group.

\subsubsection{Synthesis of sulfate diesters.}

Sulfate diesters are not uncommon throughout synthetic chemistry, for example most chemists would be familiar with dimethyl sulfate and diethyl sulfate as reagents. There are a number methods for the synthesis of such symmetrical sulfate diesters ${ }^{97-}$ 99 and their properties have been well studied. However there is very little literature precedence for the synthesis of unsymmetrical sulfate diesters. Obviously very few of the methods used for the preparation of symmetrical sulfate esters are of any applicability to the unsymmetrical compounds. This posses a very real initial challenge in the development of a sulfate monoester protecting group, how do you form an unsymmetrical sulfate diester? 
There are two basic strategies to synthesis an unsymmetrical sulfate diester of a carbohydrate: either the sulfate is introduced into the molecule using existing methods then alkylated (see Figure 5:2) or the sulfate is introduced already protected by nucleophilic attack of the carbohydrate onto a sulfate monoester containing a leaving group (see Figure 5:3).<smiles>[R]OO[R](=O)([O-])O[R]OS(=O)(=O)O[R]</smiles>

Figure 5:2: Strategy one.<smiles>[R]OS(=O)(=O)O[R]</smiles>

Figure 5:3: Strategy two.

Obviously the second approach is preferable as it allows both the sulfate and the protecting group to be introduced in a single step. However the first strategy permits the use of well established sulfation methodology. Both strategies have been employed in this development of a sulfate protecting group.

\subsubsection{Properties of sulfate diesters.}

Sulfate diesters are known are to react with nucleophiles (such as iodide ${ }^{100}$ ) and are easily hydrolysed in alkaline solutions. The hydrolysis occurs with $\mathrm{C}-\mathrm{O}$ bond fission with a $\mathrm{S}_{\mathrm{N}} 2$ type mechanism ${ }^{101}$. The sulfate monoester is a highly stable anion and as such makes an excellent leaving group (see Figure 5:4) this is a powerful thermodynamic driving force.

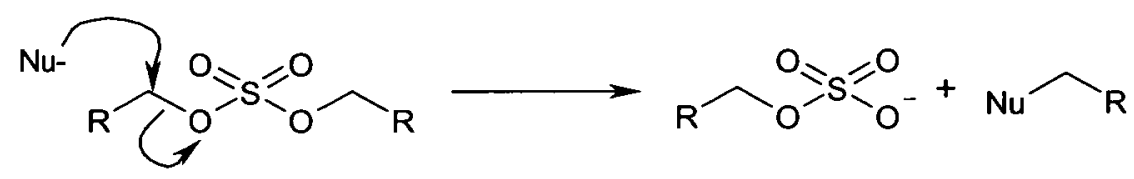

Figure 5:4: Nucleophilic susceptibility.

Due to their susceptibility to nucleophilic attack symmetrical sulfate diesters such dimethyl sulfate and diethyl sulfate are often used a powerful alkylating reagents 
(this property is also responsible for the toxicity of these compounds and their carcinogenic properties). The outcome of nucleophilic attack on such symmetrical sulfate diesters is trivial as there is only one possible set of products. However when a nucleophile attacks an unsymmetrical sulfate diester their are two potential sites for nucleophilic attack path 'a' and path 'b' (see Figure 5:5).

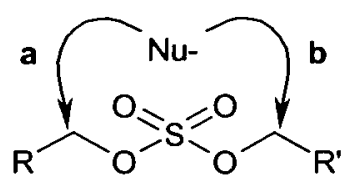

Figure 5:5: Two potential site of attack.

Where $\mathrm{R}$ is a carbohydrate, it is predicted that attack by path ' $\mathrm{a}$ ' would be discouraged for steric reasons but there are still major challenges in the design of a sulfate protecting group.

The protecting group must be chosen so that it discourages nucleophilic displacement by path ' $b$ '. This can be done in two ways, the protecting group could be bulky, preventing displacement by offering a steric hindrance to the incoming nucleophile or the protecting group could alter the electronic structure of the molecule decreasing the nucleophilic susceptibility of the carbon centre.

The protecting group must also contain a reactive handle that will control the decomposition of the sulfate diester during the deprotection step so that fission occurs exclusively between the protecting group and the sulfur centre.

After considering the known properties of sulfate esters it was clear that a small molecule protecting group that is deactivated towards nucleophilic displacement but containing a reactive "handle" to facilitate its removal was required. However before considering the selection made it is important to review the existing literature on the subject. 


\subsection{History of Sulfate Protecting Groups.}

This section examines the only two attempts to design a protecting group for carbohydrate sulfates published within the literature and discusses the strategies adopted and the failings of each approach.

The first example dates back to the 1950's and details an attempt by Foster and Hancock 72 to protect 1;2,5;6-di- $O$-isopropylidene $\alpha$-D-glucofuranose 3-(methyl sulfate) with a methyl group. The authors had already experienced the problems associated with manipulating sulfate salts and had realised that a protecting group would greatly simplify the handling of these compounds.

" Methods developed for the preparation of carbohydrate sulphates involve the direct introduction of the sulphate residue. Subsequent reactions are frequently rendered difficult by the inconvenient properties conferred by the $\mathrm{ROSO}_{2} \mathrm{O}^{-}$grouping. Thus, there is a need for methods which employ protected sulphate groups so that the salt of the free sugar sulphate can be formed in the final stages of the synthesis."

Foster and Hancock 1957.

The sulfur centre was introduced as at the sulfite oxidation level ${ }^{102}$ and then the methyl sulfite ester was oxidised with calcium permanganate to give the methyl sulfate (see Figure 5:6).
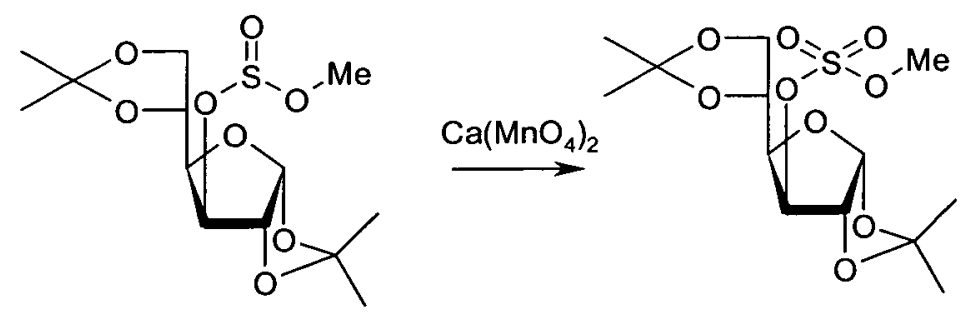

Figure 5:6: Oxidation; Sulfite to Sulfate.

The properties of this previously unknown compound were examined. The methyl sulfate ester was found to be unstable and decomposed upon storage. The methyl 
group of the 1;2,5;6-di- $O$-isopropylidene $\alpha$-D-glucofuranose 3 -(methyl sulfate) was easily deprotected to give the sodium salt of the carbohydrate sulfate by reaction with sodium iodide solution (see Figure 5:7).

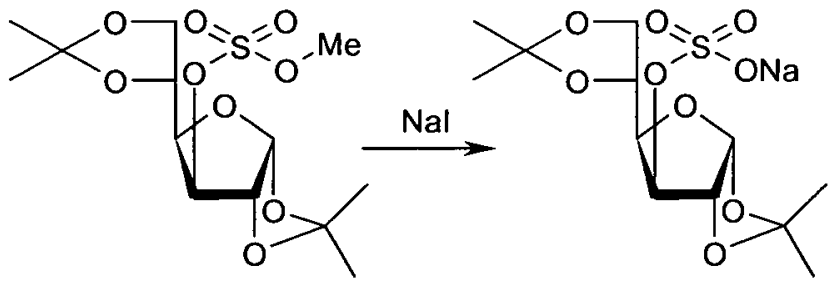

Figure 5:7: Nucleophilic susceptibility.

The compound was very easily hydrolysed by either acid or alkalis. In strong acid the product of hydrolysis was found to be D-glucose. Obviously both the sulfate grouping and the acetals are acid sensitive but it was important to determine which was the more rapidly hydrolysed. It was found that under acidic conditions the methyl sulfate was first hydrolysed to the free sulfate, then the isopropylidene acetals hydrolysed and the resulting 3 sulfo-D-glucose was hydrolysed further to give Dglucose (see Figure 5:8).

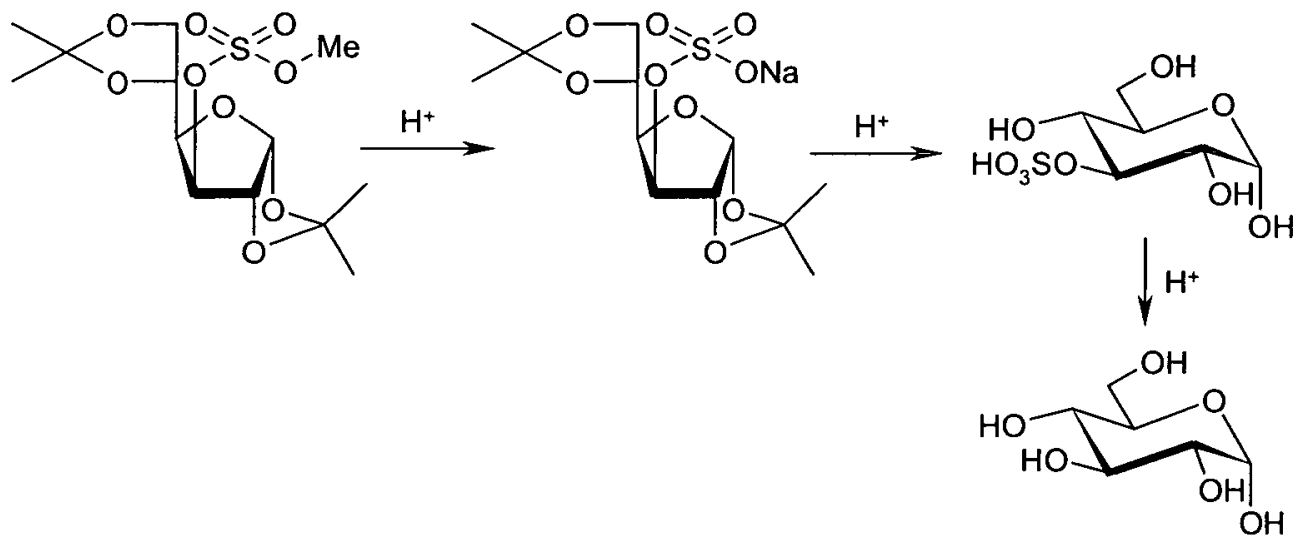

Figure 5:8:Acid hydrolysis.

It was obvious to the authors that the methyl group was far too unstable to simple reaction conditions to be of any real use and so they did not continue with the work. However they had demonstrated that for carbohydrate sulfate diesters the non carbohydrate side of the ester is the more susceptible to hydrolysis as predicted. 
The second example was published by Perlin and co-workers in the early 1980's. The authors had noted the instability of the methyl ester in the earlier work and examined two possible solutions to the problem, the protecting group was chosen so that the carbon centre attached to the sulfate was either highly non-electrophilic or highly stericly crowded. The authors also chose a different synthesis strategy. The sulfate diester was made in a single step (see Figure 5:3) by reacting the alkoxide of a carbohydrate with a chlorosulfate.

The initial attempt was to react the alkoxide of 1,2:5,6-di- $O$-isopropylidene- $\alpha-\mathrm{D}$ glucofuranose with isobutyl chlorosulfate ${ }^{103}$. It was considered that the isobutyl group would provide enough steric hindrance to nucleophilic substitution to be stable. However no product was observed when the isobutyl chlorosulfate was mixed with 1,2:5,6-di- $O$-isopropylidene- $\alpha$-D-glucofuranose in ether in the presence of pyridine (see Figure 5:9). Even had this approach been successful it is not made clear how the authors intended to remove the alkyl group from the product.

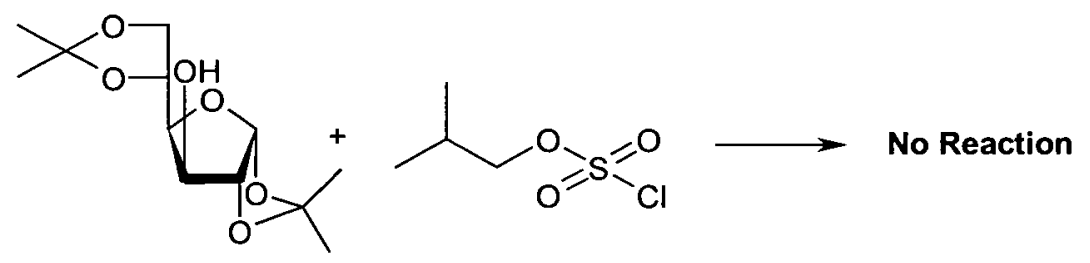

Figure 5:9: Failed diester formation.

While being unable to form a sulfate diester with the isobutyl chlorosulfate the reaction of phenyl chlorosulfate with 1,2:5,6-di- $O$-isopropylidene- $\alpha$-D-glucofuranose in THF in the presence of sodium hydride did give a good yield (75\%) of the desired product (see Figure 5:10).
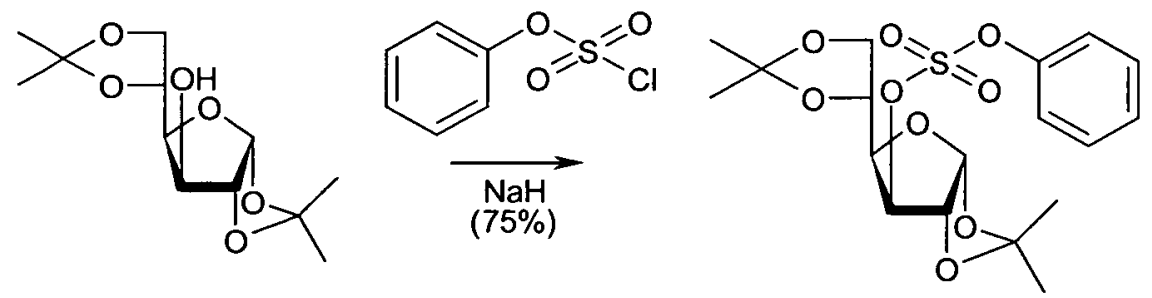

Figure 5:10: Phenyl protection. 
The intention was that due to the high electron density of the phenyl ring the sulfate diester would be resistant to nucleophilic attack. Such $S_{N} A r$ reactions are notoriously difficult to perform. This was indeed found to be the case. The compound did not decompose in solutions of sodium methoxide and was stable to various sources of fluoride ions. The phenyl group was also found to be stable to mild acidic conditions, treatment with a mixture of acetic acid and acetic anhydride led to the formation of 1,2,5,6-tetra- $O$-acetyl- $\beta$-D-glucofuranose 3-(phenyl sulfate) (see Figure 5:11).

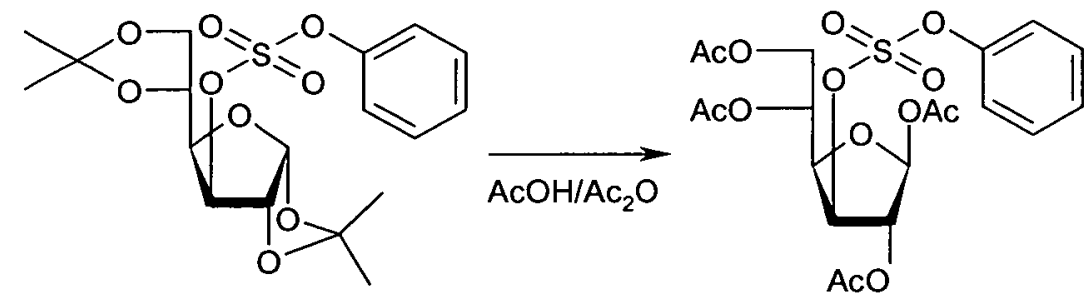

Figure 5:11: Acid stability.

The protected sulfate ester could be removed by hydrogenolysis. The phenyl group could be reduced to a cyclohexyl by hydrogenation using platinum oxide as the catalyst. The reaction was performed in the presence of potassium carbonate to act as a buffer, when the reaction is quenched with water the resulting basic solution hydrolyses the alkyl sulfate with alkyl fission giving the desired carbohydrate sulfate salt and cyclohexanol (see Figure 5:12).

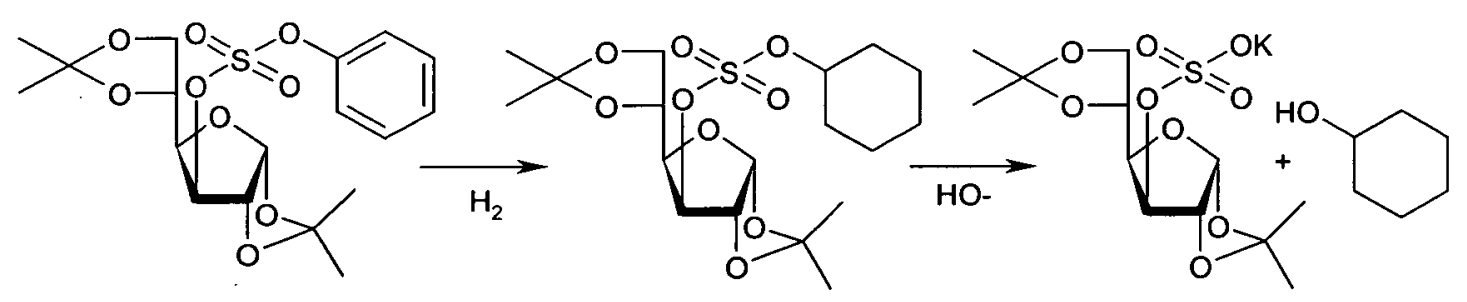

Figure 5:12: Phenyl deprotection.

While the phenyl group appears to be a suitable protecting group for sulfate esters this approach suffers a number of drawbacks. The phenyl chlorosulfate is not freely available and has to be synthesised from phenol and sulfuryl chloride which is a poor yielding reaction. Although the phenyl group is stable to common reaction conditions the deprotection step is incompatible with protecting groups that are sensitive to base and to hydrogenation. However the main problem with the use of a phenyl group as a 
protecting group is that the method of introduction is not reliable. The reaction only truly works with the two examples demonstrated in the paper. When phenyl chlorosulfate is reacted with other sugars the main product appears to be the sulfate ${ }^{92}$ (see Figure 5:13).
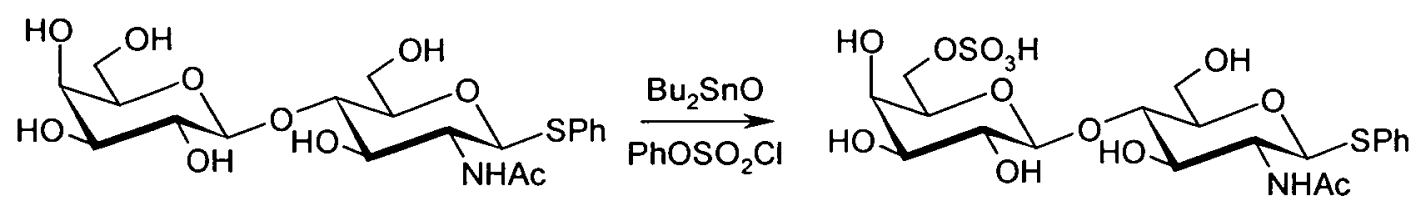

Figure 5:13: Failed introduction of phenyl sulfate.

The phenyl protecting group is not suitable for use in synthesis and its use has never been reported. Indeed Perlin has published a number of other papers where he has used the phenyl chlorosulfate reagent and has not obtained the phenyl sulfate diester as the product ${ }^{104}$.

Neither published example satisfies the criteria for a suitable sulfate protecting group. They do however establish a basis for improvement. A protecting group will be stable if it can inhibit nucleophilic displacement but the question of how to remove such a grouping still remains.

\subsubsection{Design of a protecting group.}

The reasoning behind the design of a sulfate protecting group is similar to that associated with phosphate protection in oligonucleotide chemistry. Indeed phenyl phosphoric triesters are commonly used to avoid the problems of handling anionic phosphate diesters. This therefore was a rich source of known chemistry to search for potential sulfate protecting groups.

One group that is often used in oligonucleotide chemistry that had promise was the 2,2,2-trichloroethyl group. The 2,2,2-trichloroethyl protecting group is also commonly used to protect carboxylic acids (a role first utilised by Woodward in his Cephalosporin C synthesis ${ }^{105}$ ). Trichloroethyl esters (of either carboxylic acids or 
phosphates) are inert to nucleophiles, and can easily be removed by treatment with zinc metal (see Figure 5:14).

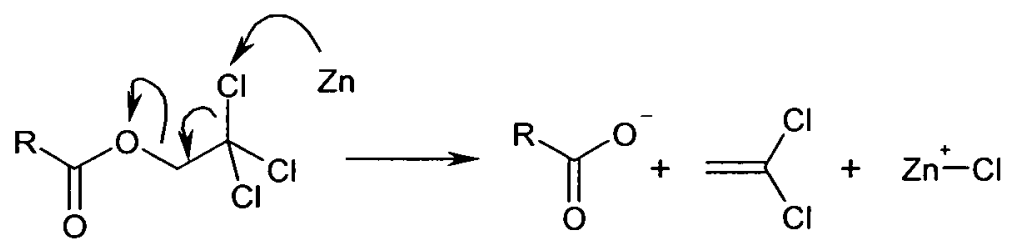

Figure 5:14: Removal of trichloroethyl group.

The stability and the ease of deprotection made the trichloroethyl group a suitable starting point from which to develop a sulfate protecting group.

A trichloroethyl sulfate should be stable to nucleophiles for two main reasons. The chlorine atoms are large and offer a significant steric obstacle to approaching nucleophiles (see Figure 5:15).
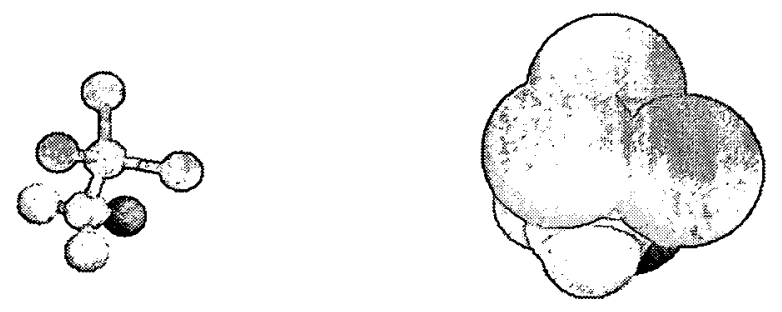

Figure 5:15: Steric bulk of trichloroethyl group.

However more important is the effect the three chlorine atoms have on the transition state of the reaction. The substitution of sulfate diesters by nucleophiles is proposed to occur by an $\mathrm{S}_{\mathrm{N}} 2$ mechanism ${ }^{99}$ and to understand the stability of these compounds it is necessary to examine the progress of this mechanism. The sulfate is an excellent leaving group due to delocalisation of the negative charge created (see Figure 5:16).

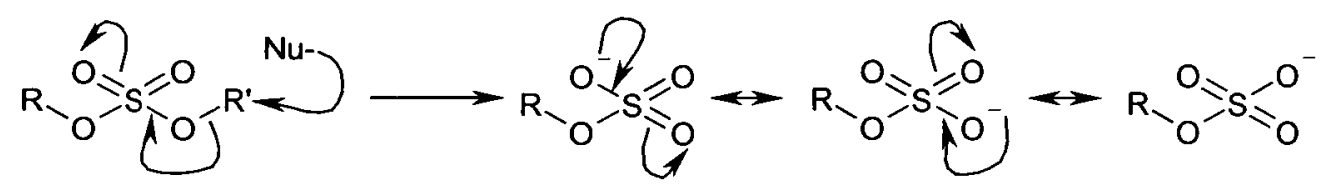

Figure 5:16: Dispersion of charge. 
This ability to stabilise a negative charge causes a distortion in the $\mathrm{S}_{\mathrm{N}} 2$ transition state. The sulfate leaves more readily than the nucleophile approaches. This the lack of symmetry leads to a partial build up of positive charge on the $\alpha$ carbon atom (see Figure 5:17).

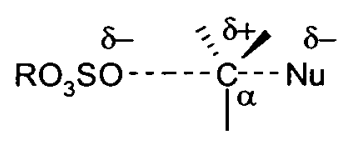

Figure 5:17: Distortion of transition state.

A comparison of the transition state of a ethyl sulfate ester with that of 2,2,2trichloroethyl sulfate ester explains the greater stability of the halogenated compound (disregarding steric/solvation complications). The methyl group of the ethyl ester is slightly electron donating and serves to stabilise the partial carbonium ion formed in the transition state however the trichloromethyl group of the trichloroethyl ester is highly electron withdrawing substantially destabilising the transition state of the displacement (see Figure 5:18). This destabilisation leads to a higher energy barrier for the displacement of the trichloroethyl group and consequentially a greatly decreased rate of reaction compared to that of the ethyl ester.
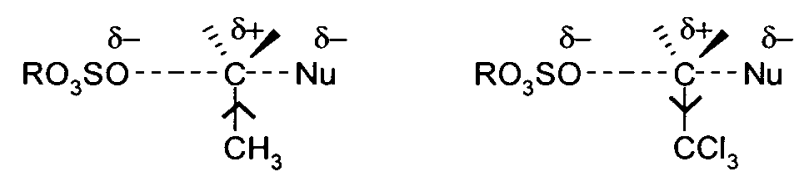

\section{Figure 5:18: Ethyl verses Trichloroethyl.}

This effect is not restricted to chlorine atoms, other halogen atoms will operate in the same fashion to a greater or lesser extent depending on their electronegativity. Fluorine atoms having the highest electronegativity should have the greatest effect on the stability of the sulfate ester. Initially this work focused on the development of the trichloroethyl group as a protecting group for sulfate esters because of it ease of removal. However it was realised from the outset that a trifluoroethyl group or an ethyl group with a mixture of chlorine and fluorine substituents would have an even greater stability than the trichloro form. 
The 2,2,2-trichloroethyl group was chosen as the initial target for this project. However a method for the synthesis of 2,2,2-trihaloethyl esters of carbohydrate sulfates had to be invented before the stability of such compounds could be examined.

\subsection{Conclusion.}

This chapter has outlined the importance of sulfated carbohydrates in biology and has made some justification for the interest in such structures. It has discussed the difficulties inherent in current synthetic approaches to these compounds and how an appropriate protecting group for the sulfate ester would circumvent these problems. The desired properties of such a sulfate protecting group have been debated and the strategy adopted for identifying a potential target group has been laid out. Various methods that could be used for the synthesis of sulfate diesters have been mentioned and their advantages and disadvantages have been discussed.

What remains is to report the synthetic work done in this project. A number of routes to the synthesis of trihalo ethyl sulfate esters have been examined with eventual success. The properties of the protected sulfated carbohydrates have been thoroughly examined and some conclusions about their utility have been drawn. 
Section Two: Results and Discussion. 


\section{Preliminary Work.}

There were a number of challenges in the development of a trihalo protected sulfate diester not least of which was to invent a method for the synthesis of such compounds that were hitherto unknown. Before devoting a great deal of time and effort into this task a trial system was devised to validate the stability and ease of deprotection of the target compounds.

\subsection{Trihaloethyl Sulfonates.}

To test out the theory that trihaloalkyl groups were stable protecting groups for sulfate esters a series of trihalo ethyl esters of para-toluenesulfonic acid were synthesised. While it was understood that sulfonates were not going to have identical properties to the corresponding sulfates, they were easy to synthesise and it was felt that their chemistry should be similar enough to test out the theory and determine which combinations of halogen atoms would be stable.

Three trihaloethyl esters of para-toluene sulfonic acid were made. Toluene-4sulfonic acid 2,2,2-trichloroethyl ester (1) and toluene-4-sulfonic acid 2,2,2trifluoroethyl ester (2) were easily prepared from the commercially available alcohols and toluene-4-sulfonyl chloride in the presence of pyridine (see Figure 6:1). Toluene4-sulfonic acid 2-chloro-2,2-difluoroethyl ester (3) was made in a similar fashion; however 2-chloro-2,2-difluoroethanol (4) was not commercially available and had to be prepared by the reduction of methyl 2-chloro-2,2-difluoroacetate ${ }^{106}$ (5). This reduction was best performed using sodium borohydride as lithium aluminium hydride, the reagent commonly used to reduce esters to alcohols, also reduces the carbon-chlorine bonds. Sodium borohydride is not normally considered a powerful enough reducing agent to reduce esters; however in this case the ester is activated due to the powerful electron withdrawing effect of the halogens. The reducing agent is also activated by pre-forming a reactive complex by refluxing sodium borohydride 
and methanol together. It was found that the chloro-difluoro compounds were less stable to column chromatography and heat than the other two tri-halo compounds.<smiles>[X]C([X])([X])CO</smiles>

$$
(54 \%) \mid \begin{aligned}
& \mathrm{NaBH}_{4} \\
& \mathrm{MeOH}
\end{aligned}
$$<smiles>COC(=O)C(F)(Cl)Cl</smiles>

(5)<smiles>Cc1ccc(S(=O)(=O)O)cc1</smiles>

$(30-60 \%)$<smiles>[X]C([X])([X])COS(=O)(=O)c1ccc(C)cc1</smiles>

(1) $\mathrm{X}=\mathrm{Cl}, \mathrm{Cl}, \mathrm{Cl}$

(2) $X=F, F, F$

(3) $X=C l, F, F$

\section{Figure 6:1: Synthesis of trihaloethyl sulfonate esters}

As discussed previously, an important criteria for any potential sulfate protecting group is that it should be inert to a variety of reaction conditions. The initial concern was that the trihalo-ethyl esters would not be stable to alkaline conditions. The stability of the three tosyl esters to a solution of sodium methoxide was compared.

Equal quantities of the three sulfonate esters were dissolved in three portions of deuterated methanol to which five equivalents of sodium had been added. The solutions were then monitored by $200 \mathrm{MHz} \mathrm{nmr}$ at regular intervals. The results are shown in Figure 6:2.

The results clearly show that (1) which contains the trichloroethyl group is the most easily hydrolysed and that (3) and (2) which contain fluorines are of similar stability and are less readily hydrolysed than (1). The results do show, that all three trihaloethyl esters are reasonably stable to sodium methoxide over the time period of $10-15$ mins that is typically required for the removal of acetate protection of hydroxyl groups in carbohydrate chemistry. The results also suggest that, although stable to hydrolysis over short periods of time, all three of the alkyl groups are sensitive enough to sodium methoxide that this is a potential method for removal at the deprotection stage.

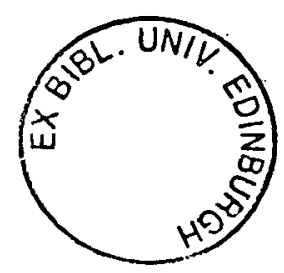




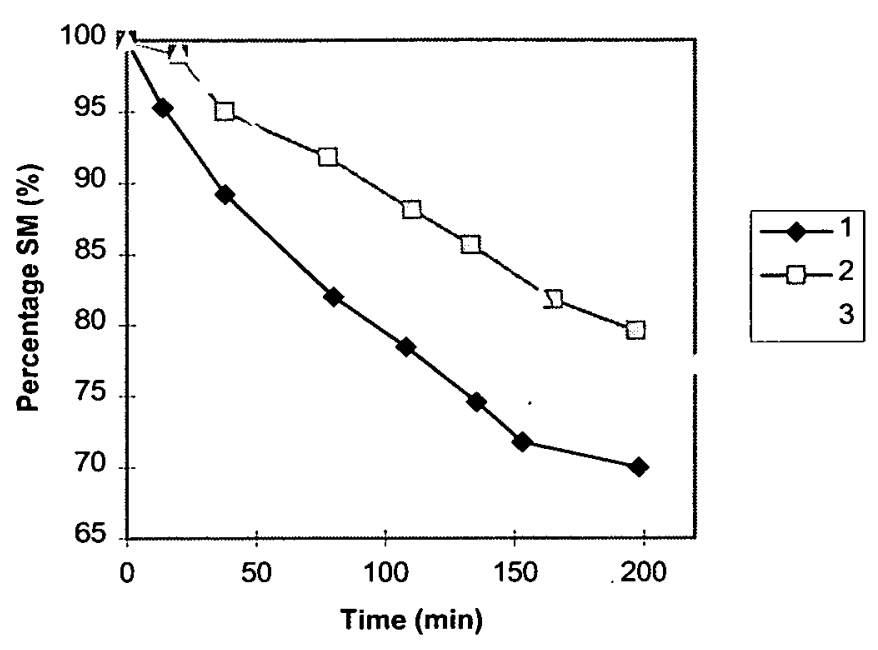

Figure 6:2: Base susceptiblity of tosyl esters.

The compounds all exhibited the required stability so it was important to explore potential methods of removing each protecting group. Since the philosophy of using a trihaloethyl protecting group stems originally from Woodward's paper on Cephlasporin $\mathrm{C}^{105}$ it was of interest to see if these groups could be cleaved with zinc metal. Each of the three tosyl esters (3), (1) and (2) were dissolved separately in acetic acid buffered with and equal mass of sodium acetate, zinc metal powder was suspended in each mixture and the reactions monitored by TLC for the disappearance of the esters and the appearance of para-toluenesulfonic acid. Within 30mins no trichloro ester (1) could be detected however neither the chlorodifluoro (3) nor trifluoro (2) esters were observed to react, even after prolonged reaction times and heating to reflux temperatures. The decomposition of the trichloro ester (1) was the expected result but it was slightly surprising that the chlorodifluoro ester (3) could not be made to react.

These preliminary results indicate that all three trihaloethyl groups could be used as potential protecting groups however the trichloro group is the easiest to remove. The chlorodifluoro group appears to be the most difficult to handle and is therefore the 
least desirable of the three. The trifluoro group has the desired stability and is simple to handle but lacks an orthogonal removal method.

This work established the order of desirability as a protecting group to be trichloro $>$ trifluoro $>$ chloro-difluoro and this influenced further work.

\section{Development of a Synthesis of Sulfate Diesters.}

Having shown that the trihaloethyl esters of sulfates were likely to be stable, a method was required for their synthesis. The literature contains very few examples of preparative methods for synthesising sulfate diesters and these mainly cover the synthesis of symmetrical diesters or cyclic sulfates 99 . However there are three obvious strategies to make an unsymmetrical diester containing a protecting group.

- Introduce the sulfate in an already protected form.

- Introduce the sulfate in an unprotected form, then alkylate to protect.

- The sulfur core is introduced at some lower oxidation state (e.g. the sulfite) in either its protected or unprotected form and oxidised to the sulfate.

The third method has the disadvantage that it involves multiple steps. Oxidation of sulfite to sulfate has been examined as a method of sulfate synthesis as was discussed in the introductory section and the original work by Foster ${ }^{72}$ on protected sulfates used this route. In that work calcium permanganate was used as the oxidising agent and reportedly was not very efficient. Modern oxidising systems have been developed and are far more effective ${ }^{71}$ however this strategy would still involve a number of steps.

The advantage of the second strategy is that, as described previously, there are already a number of published methods for the synthesis of sulfated carbohydrates, some of which are regioselective. However this involves at least two synthetic steps 
and so is less preferable than the first strategy which allows incorporation of both the sulfate core and the protecting group in one step.

\subsection{Chlorosulfates.}

Initial work in this area was directed towards a one step route using a similar approach to the earlier work of Perlin ${ }^{107}$. The strategy was to synthesise a sulfating reagent which had the sulfur core at the same oxidation level as sulfate, that contained the halogenated ethyl group and a leaving group connected to the sulfur atom. 2,2,2-trichloroethyl chlorosulfate (6) was a known compound 108 that fitted the above description. (6) was made in good yield by reacting sulfuryl chloride with the commercially available trichloroethyl alcohol (10) in the presence of pyridine at low temperature (see Figure 7:1).<smiles>O=S(=O)(Cl)[Cl+][Cl+]</smiles>

(10)<smiles>O=S(=O)(Cl)OCC(Cl)(Cl)Cl</smiles>

(6)

Figure 7:1: Synthesis of 2,2,2-trichloroethyl chlorosulfate.

Attempts to couple this compound to a trial monosaccharide substrate met with partial success. The reaction of (6) with commercially available di-acetone glucose (7) in the presence of sodium hydride gave a low yield of desired protected sulfate (8) in an equimolar mixture with the dichloro alkene (9) (see Figure 7:2).

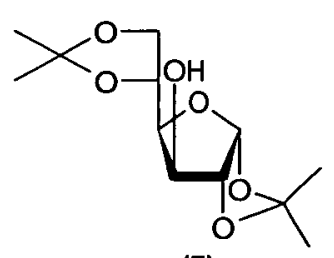

(7)

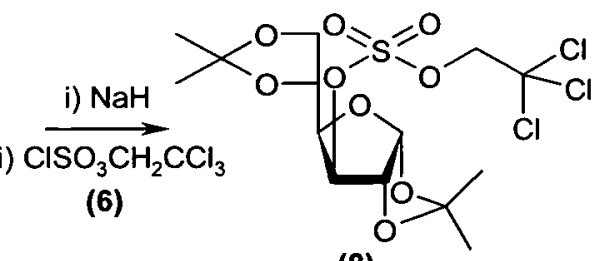

(8)

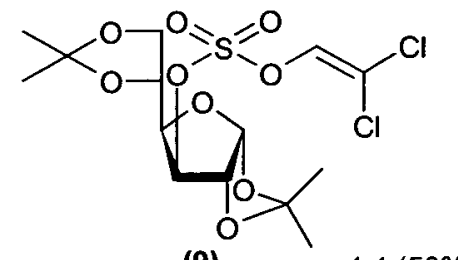

(9) $1: 1(50 \%)$

Figure 7:2: Introduction of protected sulfate.

It was not possible to separate these two compounds by chromatography and they decomposed rapidly upon heating. Treatment of the mixture with the standard zinc powder/ acetic acid mixture led to the removal of the trichloroethyl group from (8) as 
expected. However (9) was found to be stable to these conditions and did not react to give the sulfate. The conditions for the introduction of the protected sulfate were varied extensively but no improvement upon the original result could be found (see Table 7:1).

Table 7:1: Reaction of (6) with (7).

\begin{tabular}{|c|c|c|}
\hline Entry & Conditions & Result. \\
\hline 1 & Pyridine, diethyl ether & No Reaction. \\
\hline 2 & Sodium hydride, $\mathrm{THF}, 0^{\circ} \mathrm{C}$ & $23 \%$ Yield \\
\hline 3 & Potassium carbonate, acetonitrile & No Reaction. \\
\hline
\end{tabular}

When another monosaccharide (12) unit was reacted with (6) under the conditions described in Figure 7:2 further problems were observed. The product of the reaction no longer contained the trichloroethyl group and was identified as the chlorosulfate of the monosaccharide (11) (see Figure 7:3). 1;2,3;4-Di-O-isopropylidene-6chlorosulfate- $\alpha-D$-galactopyranose was a known compound 109 and the analytical data for (11) were identical.

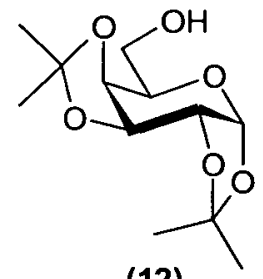

(12)

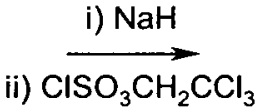

(6)

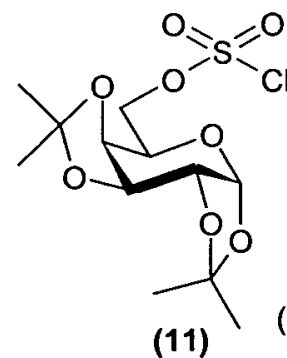

$(32 \%)$

Figure 7:3: Formation of chlorosulfate

Altering the reaction conditions had little effect upon the outcome of the experiment. Changing the base from sodium hydride to potassium carbonate, sodium carbonate, silver carbonate or silver oxide led either to no observable reaction or extensive decomposition of the monosaccharide substrate Table 7:2. 
Table 7:2: Reaction of (12) with chlorosulfate (6).

\begin{tabular}{|c|c|c|}
\hline Entry & Reaction Conditions & Result \\
\hline 1 & Sodium Hydride, THF & $\mathbf{1 1}(32 \%)$ \\
\hline 2 & Sodium hydrogen carbonate, DMF & No Reaction \\
\hline 3 & Silver carbonate, toluene & Decomposition \\
\hline 4 & Silver oxide, DMF & Decomposition \\
\hline
\end{tabular}

When the sulfation reaction was performed on 1,2,3,4-tetra- $O$-acetyl- $\beta$-Dglucopyranose (13) using pyridine as the base and solvent with a catalytic amount of dimethyl-amino pyridine (DMAP) the isolated product was identified as $1,2,3,4-$ tetra- $O$-acetyl-6-chloro-6-deoxy- $\beta$-D-glucopyranose ${ }^{110}$ (14) (see Figure 7:4). This obviously resulted from the known ability of chlorosulfates to undergo $\mathrm{S}_{\mathrm{N}} 2$ displacement with chloride nucleophiles ${ }^{111}$.

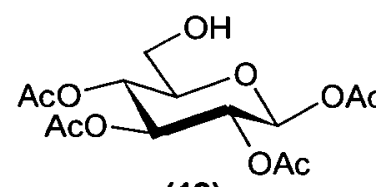

(13)

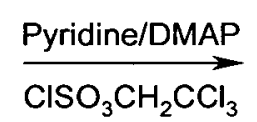

$(20 \%)$

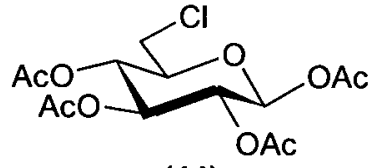

(14)

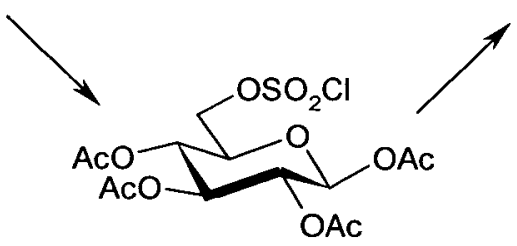

Figure 7:4: Nucleophilic displacement of chlorosulfate.

1,1'-sulfuryl diimidazole (15) has been used by Hanessian ${ }^{112}$ to form imidazylates (alkyl imidazole sulfates) and by Tewson ${ }^{113}$ to form cyclic sulfates. This reagent behaves similarly to sulfuryl chloride but is far milder. 2,2,2-trichloroethyl-1sulfonyl-1'-imidazole (16) (see Figure 7:5) would be an alternative sulfating agent to (6). It contains the trichloroethyl group, has the sulfur at an appropriate oxidation level and the imidazole is known to act as a good leaving group. (16) can be made by adding 2,2,2-trichloroethanol (10) to 1,1'-sulfuryl diimidazole (15). 
<smiles>O=S(=O)(n1ccnc1)n1ccnc1</smiles>

(15)<smiles>O=S(=O)(OCC(Cl)(Cl)Cl)n1ccnc1</smiles>

(16)

Figure 7:5: Sulfuryl imidazoles.

However (16) was a poor sulfating agent, using a mild base such as pyridine led to no reaction with the sugar substrates. Stronger bases such a sodium hydride immediately decomposed the sulfating reagent (see Table 7:3).

Table 7:3: Reaction of (13) with (16).

\begin{tabular}{|c|c|c|}
\hline Entry & Conditions & Result. \\
\hline 1 & Pyridine/DMAP, DCM & No Reaction. \\
\hline 2 & Sodium Hydride, THF, $0^{\circ} \mathrm{C}$ & Decomposition \\
\hline
\end{tabular}

Having witnessed the potential for the trichloroethyl group to act as a leaving group led to the synthesis of another reagent in this class. It was hoped that one of the alkyl groups from (17) (see Figure 7:6) would be displaced by the alkoxide of the monosaccharide to give the desired product. Compound (17) was made by heating the trichloroethyl chlorosulfate (6) with trichloroethanol in pyridine solution. However the product of the reaction of (17) with diacetone glucose (7) and sodium hydride was found to be exclusively compound (9) (see Figure 7:6).

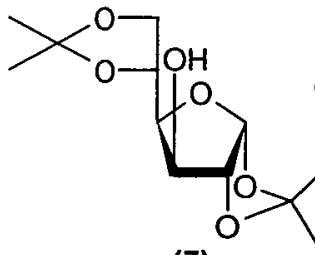

(7)

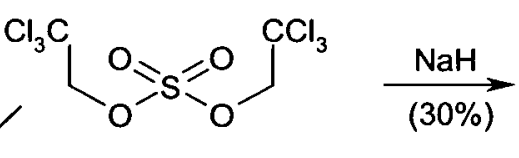

(17)

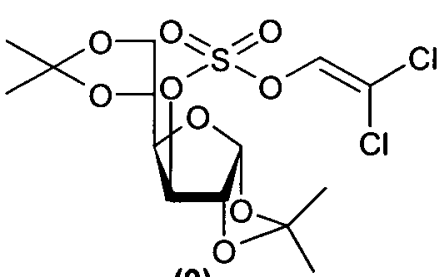

(9)

Figure 7:6:Reaction of (7) with bis-trichloroethyl sulfate (17).

An explanation for this result and for the reaction in Figure 7:2 might be as follows. The two protons on the ethyl group of (6) are quite acidic due to the electron withdrawing effect of the halogens and the sulfate. When exposed to base one of these protons is removed giving the carbanion, elimination of a chloride ion leads to 
the dichloro alkene (18). This process is rapid in strong base and seems to be as fast as nucleophilic attack of the sugar alkoxide on sulfur. Once the dehydrochlorination has taken place there is a potential enolate leaving group (see Figure 7:7). This would explain the otherwise curious result shown in Figure 7:3 where it appears that trichloroethoxide is a better leaving group than chloride.<smiles>O=S(=O)(Cl)OCC(Cl)(Cl)Cl</smiles>

(6)

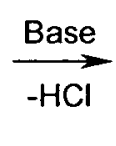

$\mathrm{HCl}$

(18)<smiles>O=S(=O)(Cl)OC=C(Cl)Cl</smiles>

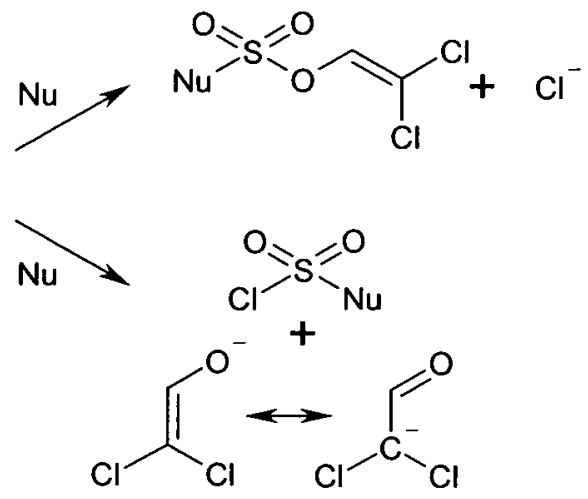

Figure 7:7: Dehydrochlorination.

There is little that can be done to avoid this problem, as the ethyl group cannot be altered to decrease the electron withdrawing effect of the halogens as this effect is the source of the stability of the sulfate esters. With the apparently insurmountable problems experienced with this approach other strategies were sought.

\subsection{Trifluoroethyl Chlorosulfate.}

Having explored all of the obvious routes to the synthesis of a trichloroethyl sulfate ester via its chlorosulfate, the possibility of making the trifluoroethyl compound via the same strategy was examined. As mentioned in the introductory section the trifluoroethyl sulfate ester should be more stable than the trichloro compound, a theory born out by the preliminary work done on the sulfonates.

Trifluoroethyl chlorosulfate (19) was made in a similar fashion to compound (6) by reacting 2,2,2-trifluoroethanol and sulfuryl chloride in the presence of pyridine at low temperature (see Figure 7:8). 


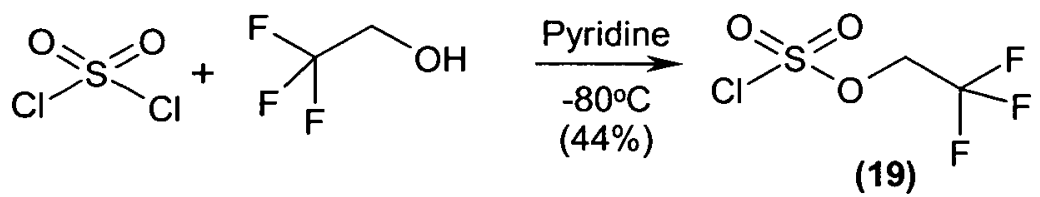

Figure 7:8: Synthesis of (19).

Using the trifluoroethyl chlorosulfate (19) to introduce a protected sulfate into compound (7) instead of the trichloro derivative resulted in a far cleaner reaction yielding (20) in a relatively good yield and without any trace of the eliminated product (see Figure 7:9).

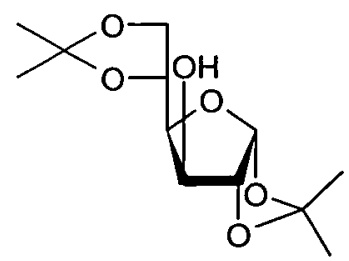

(7)

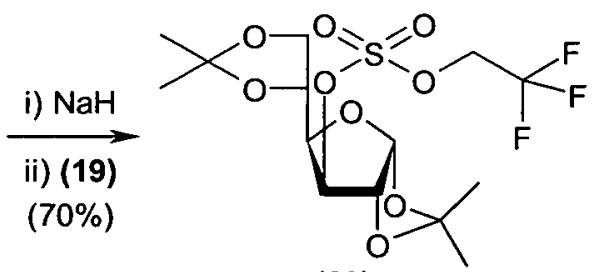

(20)

Figure 7:9: Trifluoroethyl chlorosulfate.

However once again this protocol could not be extended to other sugars. Similar results to those obtained with the use of trichloroethyl chlorosulfate were obtained when the trifluoroethyl chlorosulfate was used to sulfate other sugars.

Other than the single result shown above use of the trifluoroethyl chlorosulfate had no advantage over the trichloroethyl derivative as a method for introducing a protected sulfate group.

\subsection{Alkylation of Sulfates.}

In this section two step approaches to sulfate diesters will be described where the sulfate is first formed at the desired position in the molecule and subsequently protected.

There exists very little data on the nucleophilicity of sulfate monoesters towards carbon electrophiles nor indeed any electrophile. Given the high acidity of the sulfate 
group it is obvious that the sulfate is highly thermodynamically stable and presumably not greatly nucleophilic. It was therefore important to select powerfully electrophilic reagents to act as the alkylating component.

\subsubsection{Of sulfites and sulfates.}

Dialkyl esters of both sulfate and sulfite are known as powerful alkylating agents, (e.g. dimethyl sulfate). The bis-trichloroethyl esters of both sulfite ${ }^{114}$ (21) and sulfate $^{115}(17)$ are known and were prepared by heating trichloroethanol with either thionyl chloride or sulfuryl chloride.<smiles>O=S(OCC(Cl)(Cl)Cl)OCC(Cl)(Cl)Cl</smiles>

(21)<smiles>O=S(=O)(OCC(Cl)(Cl)Cl)OCC(Cl)(Cl)Cl</smiles>

(17)

Figure 7:10: Alkylating reagents.

Neither compound (21) nor (17) demonstrated any ability to alkylate the sulfate of diacetone glucose (22) when added to it under neutral conditions (see Figure 7:11).

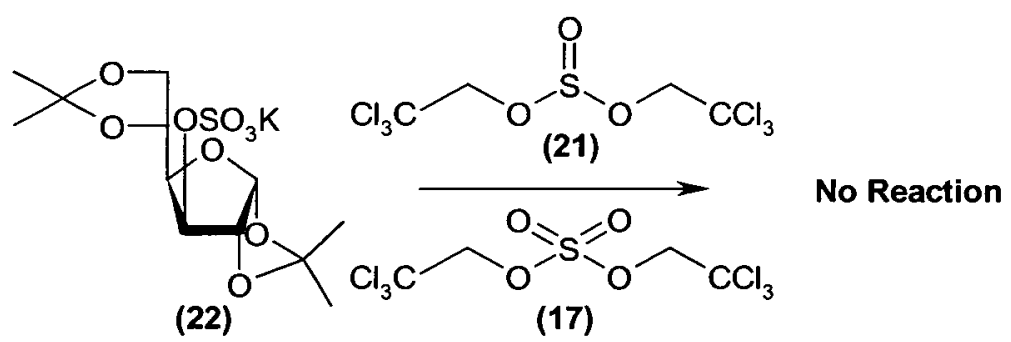

Figure 7:11: Sulfates and sulfites as alkylating agents.

With the failure of sulfate and sulfite diesters as alkylating agents other good alkylating agents were sought.

\subsubsection{Diazo compounds.}

Diazo compounds have a broad spectrum of synthetic uses and can be converted to the diazonium salts which are well known to be powerful alkylating agents. It was 
however with some reluctance that the possibility of alkylating a sulfate with a trihaloethyl diazo compound was explored.

Small molecule diazo compounds are relatively uncommon, due presumably to their propensity to decompose explosively. The compounds that are known are of restricted structural diversity and are of limited synthetic use in this project. However it was noted from an examination of the literature, that compounds with an electron withdrawing group $\alpha$ to the diazo functionality and an $\alpha$ hydrogen tend to be more stable than non electronically stabilised alkyl compounds and readily form the diazo form of the compound. This suggests that a trihalo diazoethane (from trihaloethane diazonium salt) would be more stable than diazoethane, which is highly explosive. However ALL diazo compounds have to be handled with the greatest of care and with the appropriate precautions, this will be discussed more fully later.

A thorough examination of the literature, found no references to the preparation or use of 2,2,2-trichlorodiazoethane (23). The trifluoro derivative (24) is known (see Figure 7:12) however and has been put to a number of synthetic purposes.

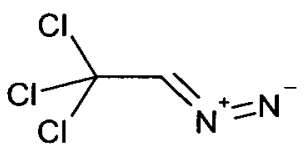

(23)

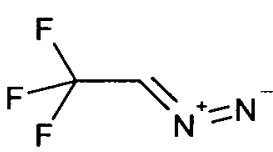

(24)

Figure 7:12: Diazo compounds.

Most importantly it has been reported that 2,2,2-trifluorodiazoethane (24) can selectively alkylate sulfonic acids in the presence of carboxylic acids. Meese ${ }^{116}$ has suggested this as a selective method for the protection of sulfonates and has shown that the trifluoroethyl group can be removed with sodium methoxide. We have already found that sulfates and sulfonates, although close cousins, do not have the same reactivity, so it was by no means certain that our work would carry on from Meese's. 
The preparation of 2,2,2-trifluorodiazoethane (24) has been reported on many occasions however most methods are variations of the original method published by Gilman and Jones ${ }^{117}$ in 1943 . However one group of workers form China ${ }^{118}$ have published an alternative route that they claim "provides an efficient and practical synthesis of 2,2,2-trifluorodiazoethane" that is "safe and convenient". This was an attractive approach and was followed up.

\subsubsection{In situ preparation of trifluorodiazoethane.}

The method for the in situ formation of trifluorodiazoethane involves the treatment of trifluoroacetyltriphenylsilane 2,4,6-triisopropylbenzenesulfonylhydrazone (25) with mild base, this precursor then undergoes extensive rearrangement to give 2,2,2trifluorodiazoethane (24). The authors suggest, the mechanism may involve a variation of the Bamford-Stevens ${ }^{119}$ reaction where the normal fragmentation of the tosyl hydrazone is preceded by an unusual base catalysed 1,3-silicon migration from carbon to nitrogen (see Figure 7:13).

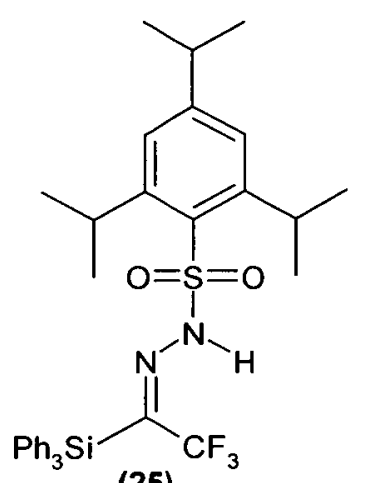

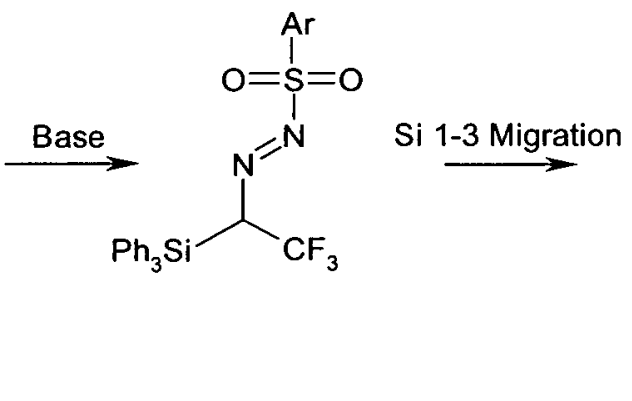

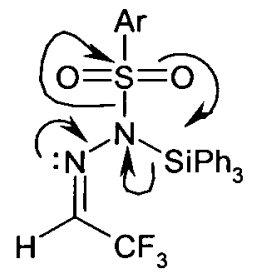

(25)<smiles>[Y8]S(=O)O</smiles><smiles>C[C@@H]1[CH]OC1</smiles>

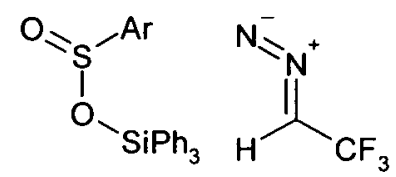

(24)

Figure 7:13: Precursor rearrangement.

Trifluoroacetyltriphenylsilane 2,4,6-triisopropylbenzenesulfonylhydrazone (25) was eventually synthesised in reasonable yield. This work was published as a short 
communication in which very little experimental detail is given. The authors state that the hydrazone (25) was prepared "from trifluoroacetyltriphenylsilane (26) and 2,4,6-triisopropylbenzenesulfonylhydrazine (27) in acetonitrile in the presence of a catalytic amount of conc. hydrochloric acid" however it has been found that (25) is rather sensitive and decomposes on exposure to acid. The reaction will only proceed if the acid is omitted.

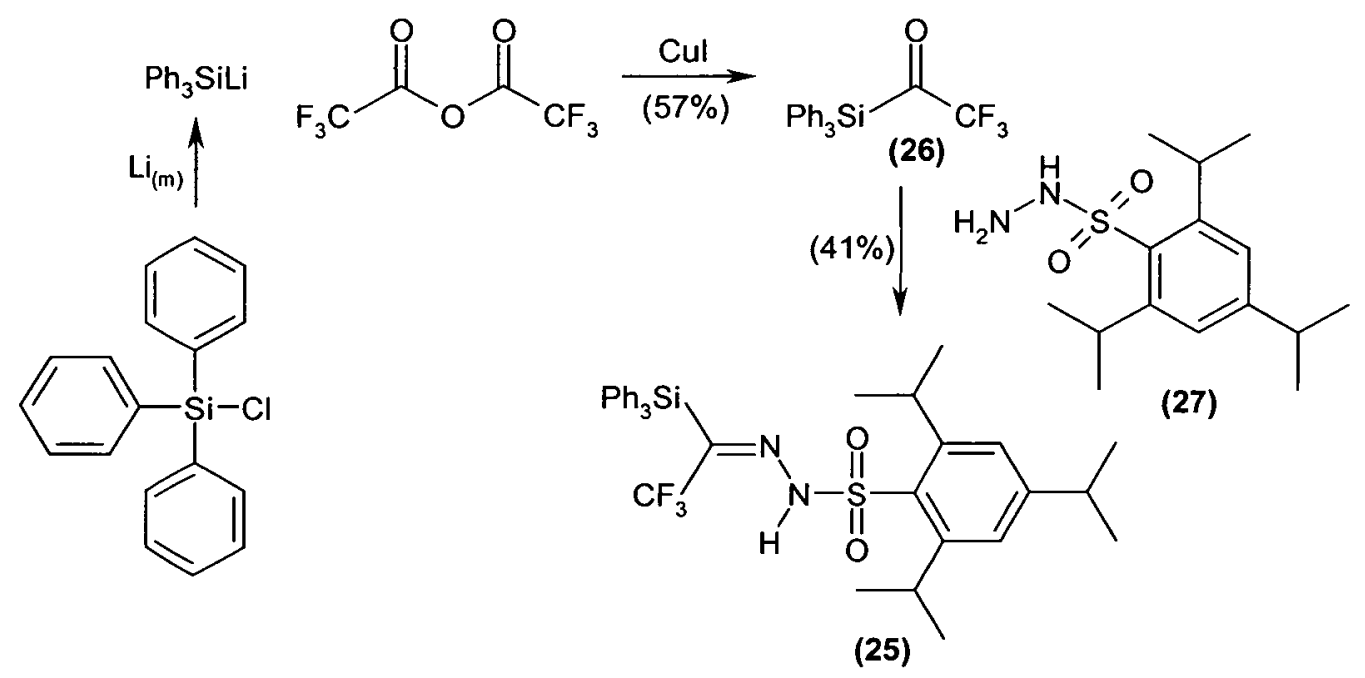

Figure 7:14: Preparation of precursor (25).

Trifluoroacetyltriphenylsilane (26) was formed by treating the silyllithium reagent formed from the addition of lithium metal to the triphenylsilyl chloride ${ }^{120}$ with trifluoroacetic anhydride in the presence of a stoichiometric amount of cuprous iodide $^{121}$. The resulting silyl ketone (26) was reacted with the sulfonyl hydrazine (27) at room temperature to give (25).

When a mild base such as trimethylamine was added to a solution of (25) in THF, a yellow coloration of the solution was observed, indicating the formation of a diazo compound. In the work of Meese, 116 discussed earlier, it had been shown that addition of a solution of 2,2,2-trifluorodiazoethane (24) to para-toluenesulfonic acid led to the formation of the 2,2,2-trifluoroethyl ester. When para-toluenesulfonic acid was added to a solution of (25) and trimethylamine in THF no reaction was observed. 
Direct addition of sulfonic acid to the solution of (25) and base is unsuccessful because the sulfonic acid readily forms an unreactive complex with the organic base.

Attempts were made to isolate the 2,2,2-trifluorodiazoethane however it would seem that the rearrangement of the silane yields only low concentrations of the diazo compound. Certainly the paper by Jin and co-workers 118 contains accounts of cycloaddition reactions between 2,2,2-trifluorodiazoethane and dipolarophiles, the reaction times of which were many times slower than when 2,2,2trifluorodiazoethane (24) is formed by standard routes ${ }^{122}$. This is borne out by the work of other groups that state that although 1-3 (carbon-nitrogen) silicon migration does occur it is slow process 123 .

\subsubsection{Nitrosation of trifluoroethylamine.}

The method most commonly used to prepare 2,2,2-trifluorodiazoethane (24) is that first published by Gilman and Jones ${ }^{117}$. This preparation involves the addition of an aqueous solution of sodium nitrite to a cooled aqueous solution of 2,2,2trifluoroethylamine hydrochloride (28) (see Figure 7:15). The diazo compound is subsequently isolated by an ethereal extraction.

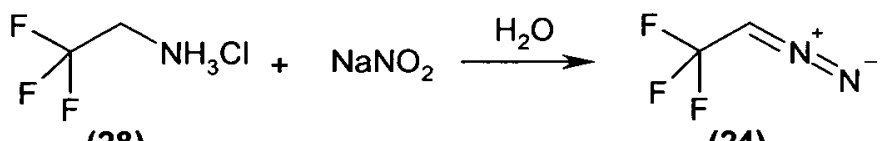

(28)

Figure 7:15: Nitrosation of 2,2,2-trifluoroethylamine.

This method of preparation is no longer satisfactory as it involves extensive manual handling of potentially lethal intermediates. In this project a further adaptation of the modified procedure published by Ho and Fishbein ${ }^{124}$ was used. The diazo compound was collected by passing a stream of inert gas through the aqueous solution of sodium nitrite and amine. The gas stream was subsequently passed across potassium hydroxide pellets (to remove moisture) into a flask of cold acetonitrile. This modification allows the diazo compound to be collected safely with minimal 
handling and free from amine and water. An illustration of the apparatus used is shown in Figure 7:16. All glass joints were of the Clearfit ${ }^{\circledR}$ type, all tubing in contact with the reaction contents was made of glass with annealed ends. Tubing was inserted into reaction flasks via "screw fit" connectors each with Teflon ${ }^{\circledast}$ seals fitted.

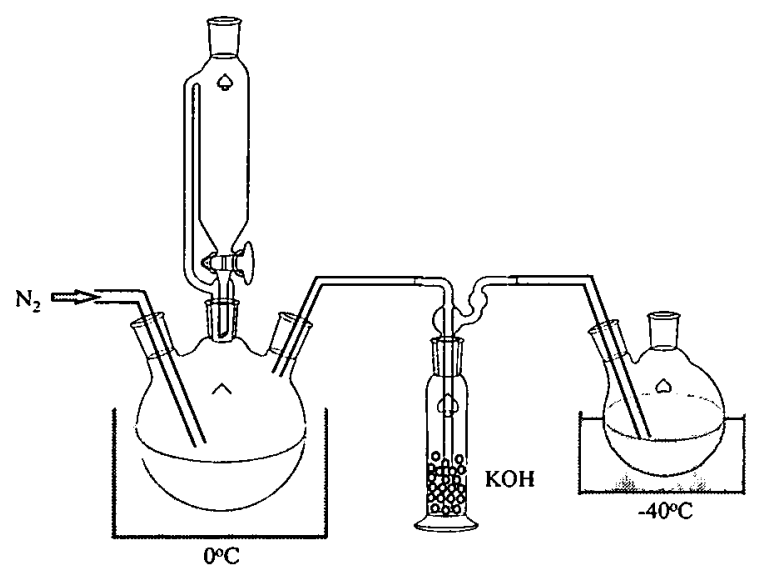

Figure 7:16: Diazo glassware.

Any cautious scientist would be concerned about the repeated synthesis and handling of a toxic and potentially explosive reagent. As previously explained 2,2,2trifluorodiazoethane should be more stable than other diazo compounds however it must still be handled with extreme care as it may be made to decompose explosively. As anecdotal evidence for this a quantity of diazo compound, too small to be seen by the naked eye, was heated with a hot air gun. The material detonated with a loud bang. The compound may be used safely if certain precautions are followed. All reaction vessels should be free of rough surfaces such as ground glass joints, cracks or chips. All work must be conducted behind blast screens and appropriate personal protective clothing must be worn. Under no circumstances should the diazo compound be isolated neat and precautions should be taken to prevent this from happening accidentally, neither should solutions of the compound be stored for any length of time. In general it is best to follow all practices suggested for the handling of diazomethane. If these sensible precautions are followed there is no reason why this reagent cannot successfully and safely be used for laboratory scale reactions. 


\subsection{Trifluoroethylation of Sulfates.}

As discussed in the introduction, there are a host of methods published for the synthesis of sulfated carbohydrates. A sample of the potassium salt of $1 ; 2,5 ; 6-\mathrm{di}-\mathrm{O}$ isopropylidene-6-sulfo- $\alpha$-D-glucofuranose (22) was prepared using the procedure published by Percival ${ }^{63}$ and added to a solution of 2,2,2-trifluorodiazoethane (24) in acetonitrile. After the first hour no reaction had been observed by TLC. An excess of citric acid was then added to the reaction flask. An immediate reaction was obvious, a large quantity of colourless gas (assumed to be nitrogen) came out of solution and the yellow coloration of the solution faded visibly. After a further hour the reaction was almost complete (by TLC) with a single non polar product having been formed. The reaction was stirred at room temperature for a further $16 \mathrm{~h}$ (overnight) to allow the reaction to reach completion and to allow the remaining trifluorodiazoethane to evaporate harmlessly (it hydrolyses rapidly on contact with moisture ${ }^{124}$ ). The product was isolated and identified as (20) and identical to the previously prepared material (see Figure 7:9).

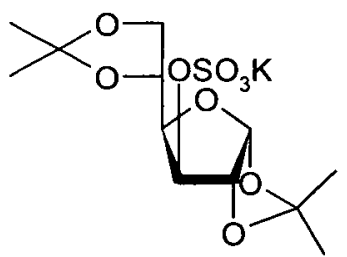

(22)

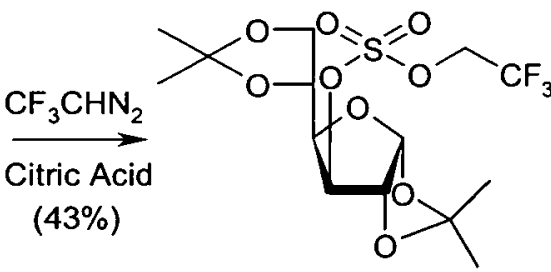

(20)

Figure 7:17: Alkylation of sulfate by 2,2,2-trifluorodiazoethane.

The necessity for the addition of citric acid becomes apparent when the stoichiometry of the reaction is examined. As shown in Figure 7:15 the nitrosation of the amine forms the diazo compound however as shown in Figure 7:18 the actual alkylating reagent is the diazonium ion which requires the addition of a proton to the diazo compound. Normally when diazo compounds such as diazomethane are used as alkylating agents the compounds they are reacted with are acids or alcohols that 
contain an acidic proton. The sulfate, being isolated in its salt form lacks this labile proton and the reaction does not proceed until it is added in the form of an acid.

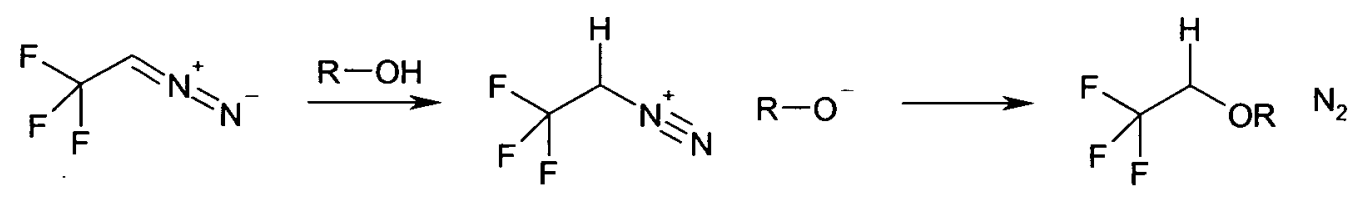

Figure 7:18: Diazo to Diazonium ion

Amazingly the 2,2,2-trifluorodiazoethane is highly selective for the sulfate monoester in preference to the citric acid which should be a better nucleophile. The reason for this might be as follows. It is believed that these alkylation reactions can occur by an $\mathrm{S}_{\mathrm{N}} 1$ type process ${ }^{125}$. The rate of reaction is independent of the concentration of the nucleophile. The rate limiting step is the formation of the carbocation which then reacts rapidly with a nucleophile (see Figure 7:19). Now while the overall rate of reaction is independent of the concentration of the nucleophile, when there are two competing nucleophiles $\left(\mathrm{X}^{-}\right.$and $\left.\mathrm{Y}^{-}\right)$the actual outcome of the reaction is determined by their relative concentrations.

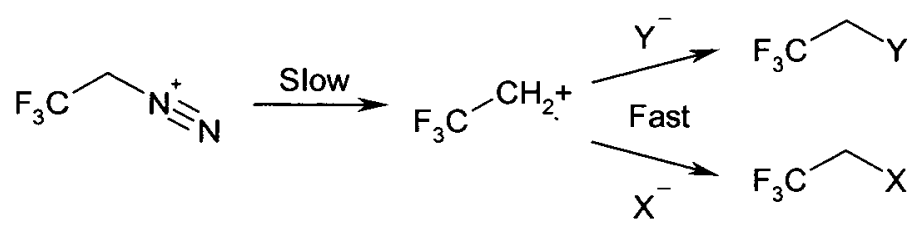

Figure 7:19: $S_{N} 1$ Displacement.

In this particular case, the sulfate monoester predominates because it has a far lower $\mathrm{pK}_{\mathrm{a}}$ than citric acid and is almost totally ionised in solution. The result of the reaction is that the sulfate diester is formed in preference to an ester of citric acid. Some small amount of citric acid esters are produced (observed as polar spots on TLC) but as citric acid is trivalent these are easily removed by aqueous extraction.

The conditions of the sulfate alkylation step were varied to identify possible improvements to the yield. 
The reaction solvent was altered by adding quantities of other solvents to the reaction mixture. Solvents tested were methanol, water, DMF and diethyl ether. The results were quite clear, the presence of any other solvent other than acetonitrile drastically reduces the yield. The reason for this is not obvious, acetonitrile is polar enough to encourage the dissociation of the diazonium salt (unlike diethyl ether) but is not highly solvating (like DMF, methanol and water). This may be an important balance.

The proton source was changed from citric acid to acetic acid with the result that the yield was reduced.

\section{Synthesis of Protected Sugar Sulfates.}

A reliable method for alkylating sulfate monoesters with the trifluoroethyl group has been devised. This section illustrates that this method is general and is tolerant of functional group diversity.

\subsection{Protection of Sulfated Monosaccharides.}

A number of sulfated monosaccharides were synthesised and reacted under the standard alkylation conditions described in section 7.4. A selection of sulfates were chosen to test the methods tolerance to other functionality and to the position of sulfation.

As discussed in the introductory chapter there are many published sulfation strategies. However as detailed in the previous section the alkylation reaction is quite sensitive to traces of solvents other than acetonitrile. To avoid the problematic step of purifying the sulfated monosaccharide before protecting the sulfate a two step one pot method was developed. The carbohydrate was sulfated in acetonitrile and the alkylation conducted without any intermediate purification. The monosaccharides were sulfated with sulfur trioxide available as a complex either with pyridine or trimethyl amine. Both these complexes are commercially available as colourless solids which are simple and safe to handle. 
All monosaccharides chosen were reacted under identical conditions the only variations being in the conditions of the sulfation step. No attempt was made to "fine tune" the reaction conditions for each substrate as a general and robust reaction was being sought.

\subsubsection{Protected monosaccharides.}

By this route $1 ; 2,5 ; 6-\mathrm{di}-O$-isopropylidene- $\alpha$-D-glucofuranose $6-\left(2^{\prime}, 2^{\prime}, 2^{\prime}-\right.$ trifluoroethyl sulfate) (20), as previously synthesised, was produced with a yield of $51 \%$ over the two steps (see Figure 8:1). Although acceptable this yield was not as high as obtained by the chlorosulfate route (see Figure 7:9) but higher than the previous two step synthesis where the sulfate was isolated (see Figure 7:17).

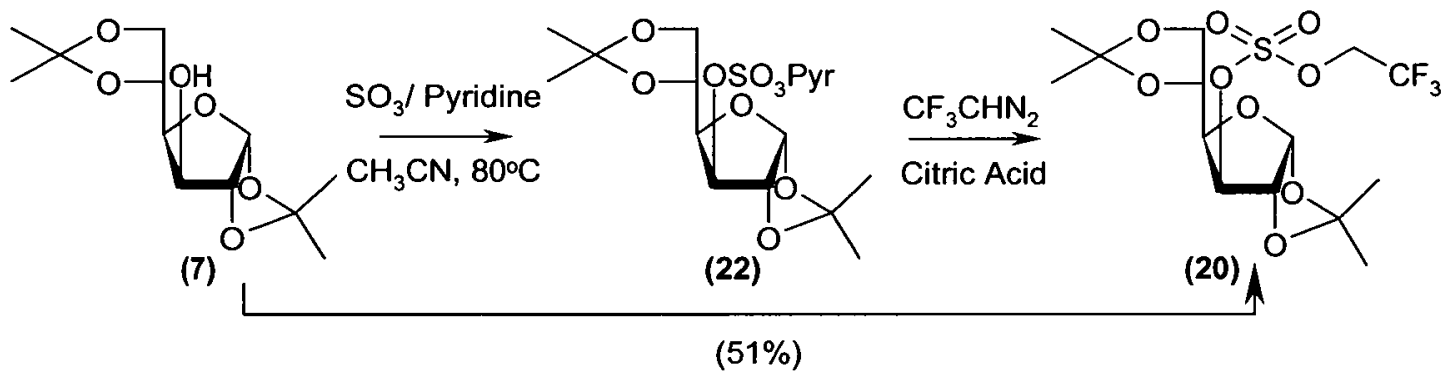

Figure 8:1: Two step synthesis of (20).

In previous attempts it had not been possible to form protected sulfates at the 6position of either 1,2,3,4-tetra- $O$-acetyl- $\beta$-D-glucopyranose (13) or $1 ; 2,3 ; 4$-di- $O$ isopropylidene- $\alpha$-D-galactopyranose (12). Both compounds were sulfated and alkylated in good yield using this procedure (see Figure 8:2 and Figure 8:3).

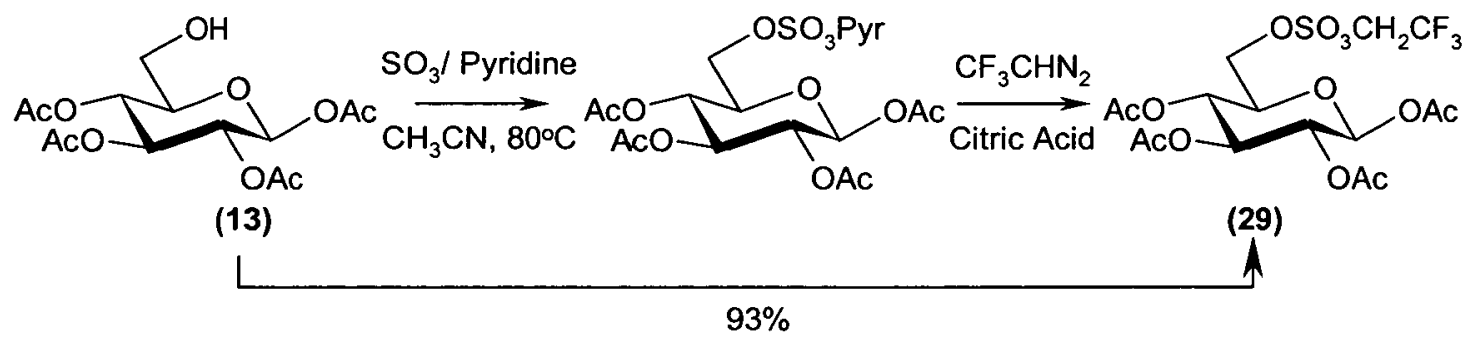

Figure 8:2: Synthesis of (29) 


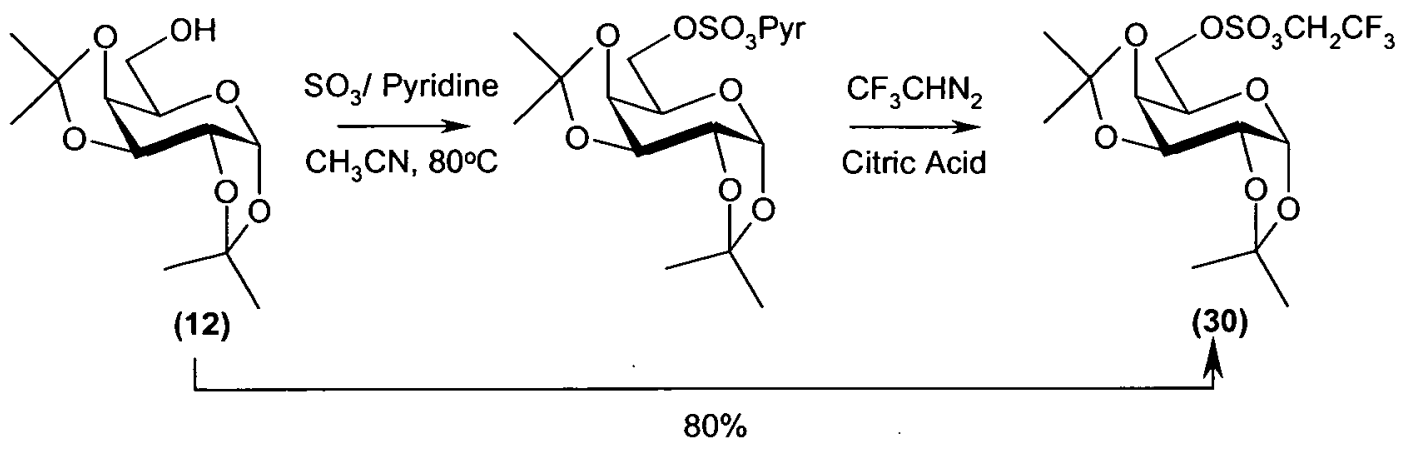

Figure 8:3: Synthesis of (30)

Other secondary alcohols could be sulfated and alkylated successfully using this procedure. Protected sulfates at the 2 and 4 positions were prepared to complete this demonstration of the procedures potential. Neither of the substrates for these examples were available commercially however they were easily prepared.

1,3,4,6-tetra- $O$-acetyl- $\alpha$-D-glucopyranose (31) can be prepared from inexpensive Dglucose monohydrate ${ }^{126}$ (see Figure 8:4).

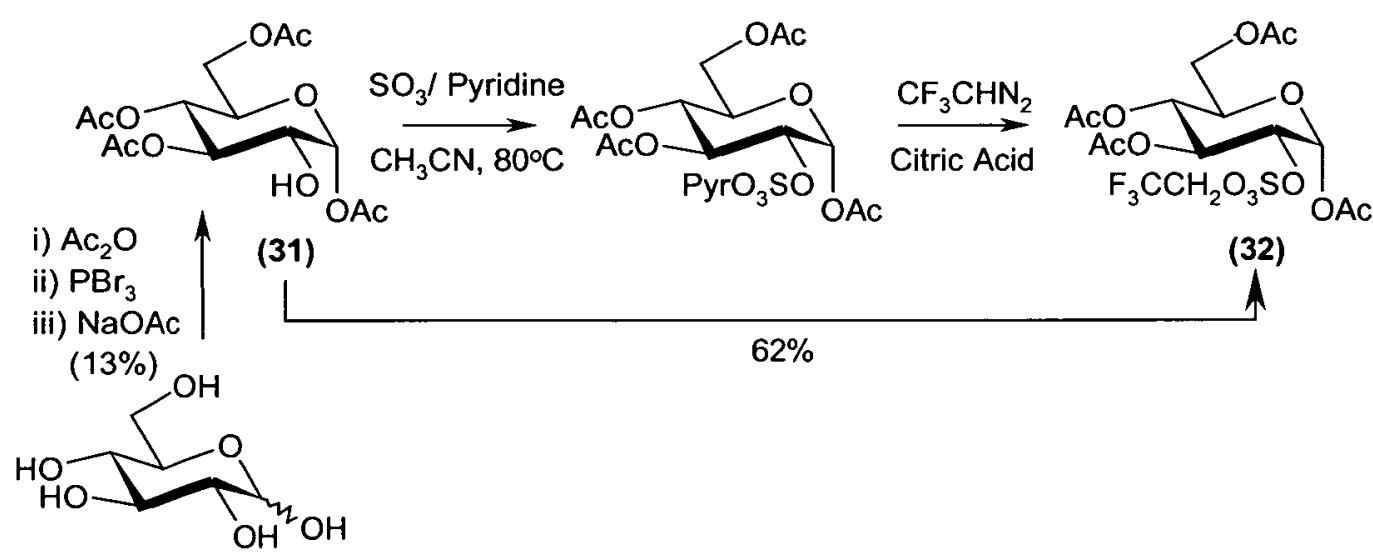

Figure 8:4: Synthesis of (32)

The sugar is per-acetylated, then the anomeric acetate is converted to a glycosyl bromide by treatment with phosphorus tribromide. Addition of an aqueous solution of sodium acetate promotes displacement of the bromide and neighbouring group participation by the acetate at the 2-position. The resulting acetoxonium ion is trapped by water to form an ortho ester ${ }^{127}$, rearrangement of which returns the acetate to the anomeric position (see Figure 8:5). 


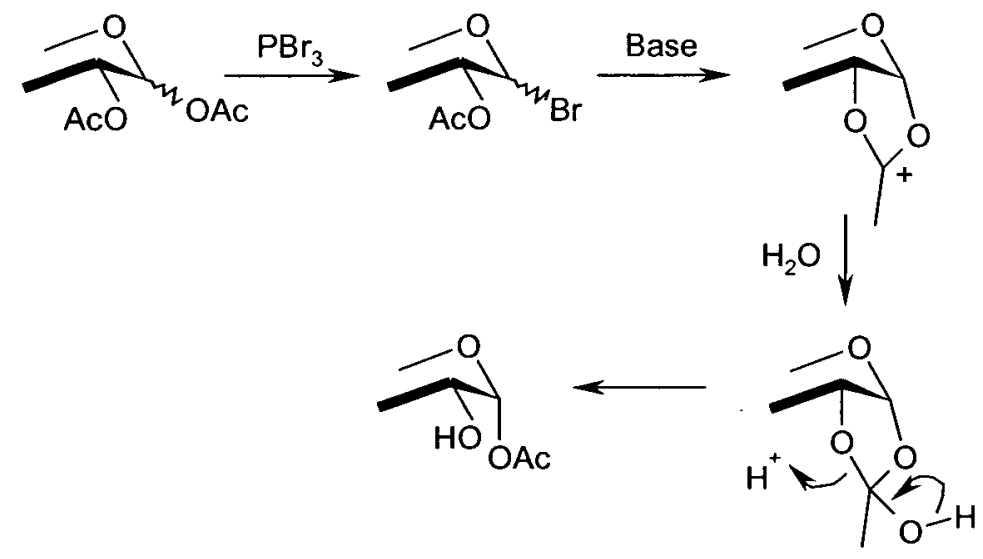

Figure 8:5: Acetate migration.

The selectively protected compound (31) was sulfated and protected under the standard conditions giving a reasonable yield (62\%) of 1,3,4,6-tetra-O-acetyl- $\alpha-\mathrm{D}-$ glucopyranose 2-(2',2',2'-trifluoroethyl sulfate) (32) (see Figure 8:4).

Subtle differences in the reactivity of the hydroxyl groups of a sugar can often be exploited. These differences allow the 2, 3 and 6 positions of methyl $\alpha$-Dglucopyranoside (33) to be selectively protected leaving the 4-position untouched (see Figure 8:6).
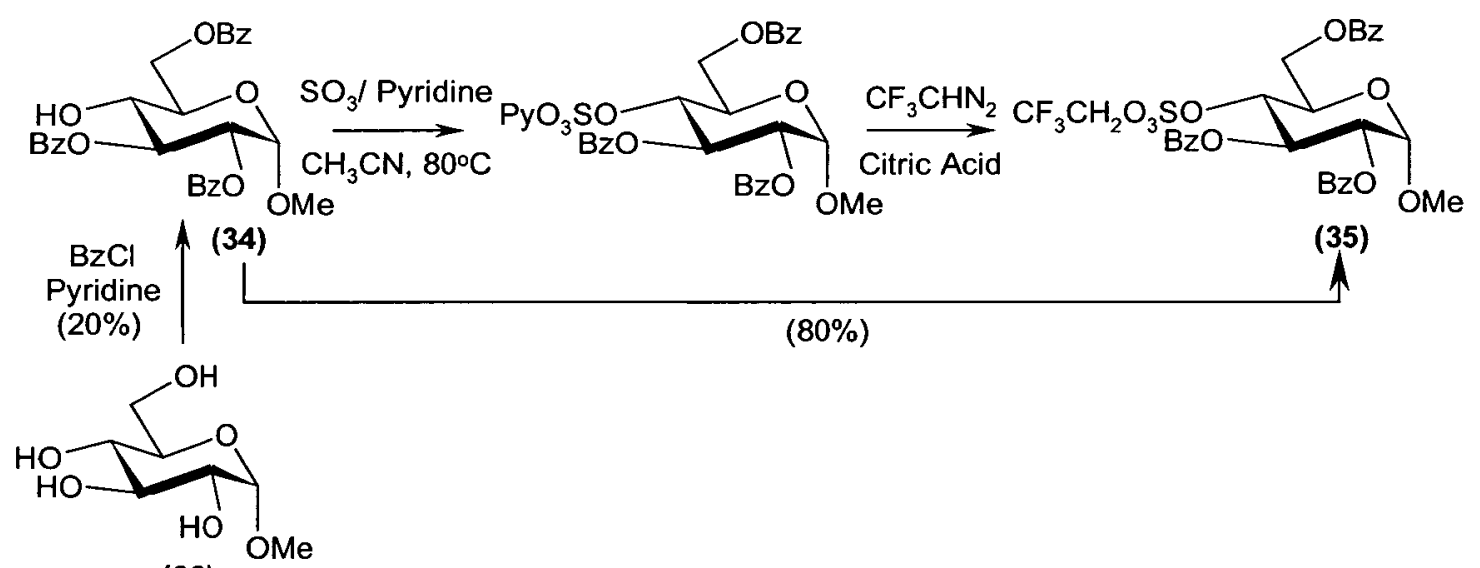

Figure 8:6: Synthesis of (35).

Methyl 2,3,6-tri- $O$-benzoyl- $\alpha$-D-glucopyranoside (34) is one of the main products of the careful benzoylation of methyl $\alpha$-D-glucopyranoside with 3 equivalents of benzoyl chloride ${ }^{128}$. The product could then be sulfated and protected in the usual 
manner to give methyl 2,3,6-tri-O-benzoyl- $\alpha$-D-glucopyranoside 4-(2',2',2'trifluoroethyl sulfate) (35) (see Figure 8:6) in excellent yield (80\%).

The only ring position that could not be successfully sulfated and alkylated was the anomeric position. Although 2,3,4,6-tetra- $O$-benzyl-D-glucopyranose (36) could be sulfated 81 (to give (37)) when it was treated with the trifluorodiazoethane solution the isolated product was found to be a mixture of the original starting material and the acetimidate (38) (see Figure 8:7).

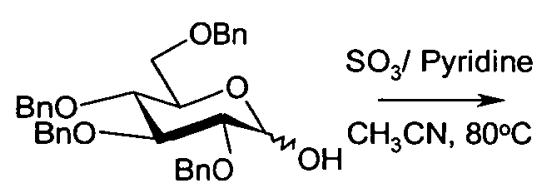

(36)

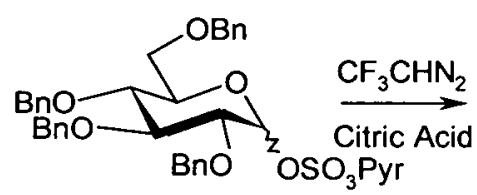

(37)

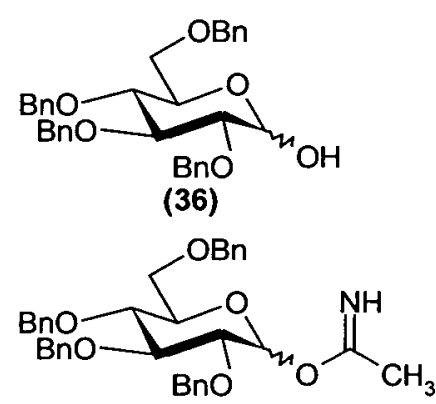

(38)

Figure 8:7: Failed protection of anomeric sulfate.

Obviously the protected anomeric sulfate is too easily hydrolysed by acid to be isolated explaining the recovery of alcohol (36). The formation of the acetimidate (38) is explained by the Pinner reaction ${ }^{129}$ (see Figure 8:8).

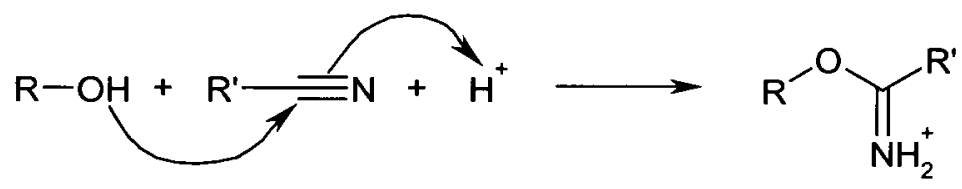

\section{Figure 8:8: The Pinner reaction.}

Hydrolysis of the protected sulfate leads to the formation of 2,2,2-trifluoroethyl sulfuric acid monoester a strong acid which initiates alcoholysis of acetonitrile.

\subsubsection{Selective sulfation.}

As discussed in the introduction sulfates may be introduced into unprotected sugar selectively using stannylene acetal chemistry. Two examples were chosen to 
illustrate that selective sulfation can be used in conjunction with the protection procedure. Methyl 3-sulfo- $\beta$-D-galactopyranoside 92 (39) and methyl 4;6-Obenzylidene-2-sulfo- $\alpha$-D-glucopyranoside 93 (40) were made via their dibutyltin acetals using published procedures. Careful, complete removal of the solvent was the only purification performed on the intermediate sulfate. The protection step went in reasonable yields to give the desired products (41) and (43) see Figure 8:9 and Figure $8: 10$.

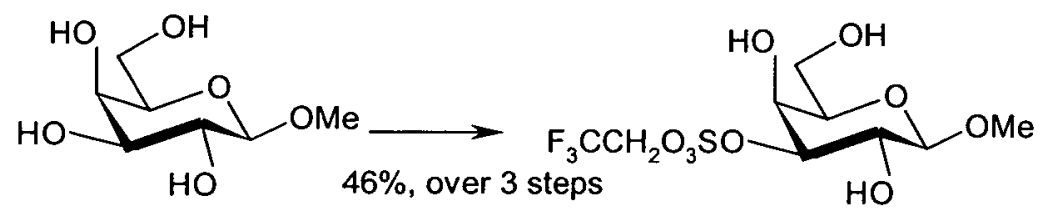

(42)

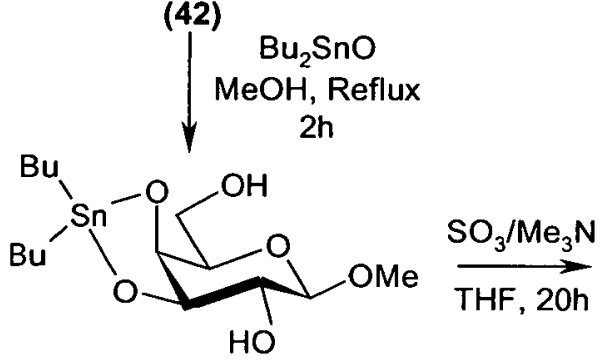

(41)

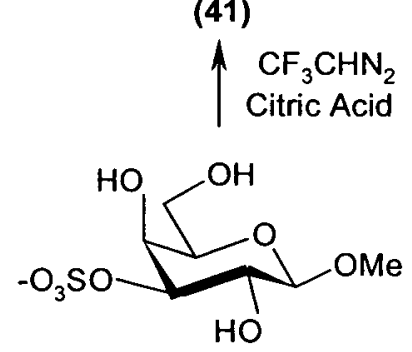

(39)

Figure 8:9: Synthesis of (41).

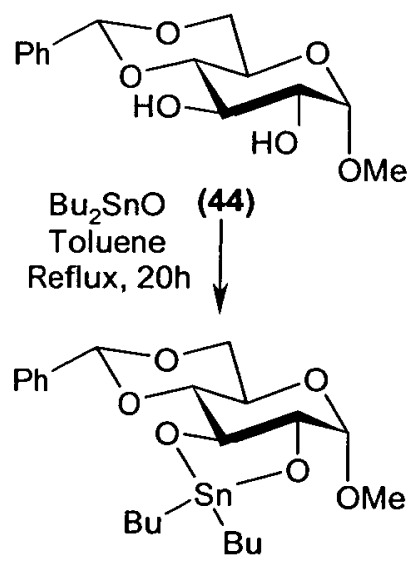

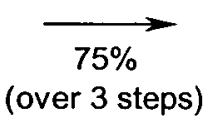

(over 3 steps)

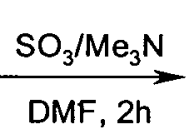

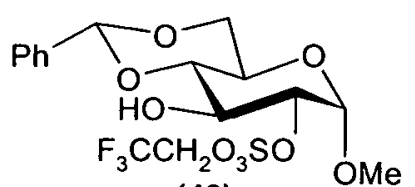

(43)
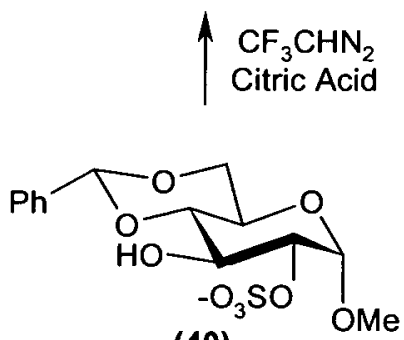

(40)

Figure 8:10: Synthesis of (43).

It would appear that the presence of other reagents does not interfere with the action of the trifluorodiazoethane. 


\subsection{Disaccharides.}

To further test the robustness of the protection procedure, a sulfated disaccharide was protected to illustrate that the reaction conditions would not affect the glycosidic linkage. Phenyl 1-deoxy-4-O-(3'-O-sulfo- $\beta$-D-galactopyranosyl)-1-thio- $\beta$ glucopyranoside, sodium salt (45) was available from previous work done by the Flitsch group ${ }^{91}$ and was selectively sulfate protected to give (46) in reasonable yield (see Figure 8:11).
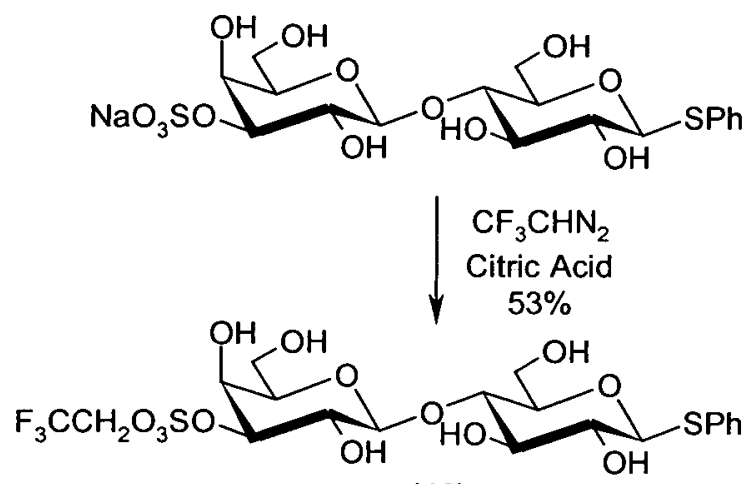

(46)

Figure 8:11: Disaccharide protection.

This example also demonstrates that the protection methodology is compatible with thio glycosides. This compatibility is important if protected sulfates are to be incorporated into synthesise of larger sugar structures.

\subsection{Conclusions.}

The sulfate protection method has been found to be extremely robust. It works well for sulfates at almost any position on the sugar ring and it is tolerant of a diversity of functionality. It does not affect glycosidic linkages and can be applied to thio glycosides which are important carbohydrate building blocks. One point that should be noted is that yields tend to be highest when the sulfated compound has no free hydroxyl groups and contains no acid sensitive functionality. Although the trifluoroethyl protection step is performed under mildly acidic conditions the sulfur trioxide sulfation step is highly acidic and if extremely acid sensitive molecules are to be used it would be best to consider other sulfation methods. 


\section{Stability of Sulfate Protecting Group.}

As discussed in the introduction the sulfate protecting group is only of use if it is stable to common reaction conditions. This section demonstrates that the trifluoroethyl group is stable to reaction conditions used to remove some common protecting groups. A number of example monosaccharides were chosen, their protecting groups removed and the product examined for the presence of the sulfate and the trifluoroethyl group.

\subsection{Acid Stability.}

Acetals of one type or another are commonly used in carbohydrate chemistry. There are a number of different reaction conditions known for their removal, most require an acidic reagent. Compounds 1;2,5;6-di- $O$-isopropylidene- $\alpha$-D-glucofuranose 3(2',2',2'-trifluoroethyl sulfate) (20) and 1;2,3;4-di- $O$-isopropylidene- $\alpha-D$ galactopyranose 6-(2',2',2'-trifluoroethyl sulfate) (30) were screened for stability against a cross section of conditions known to remove isopropylidene acetals. Both compounds were inert to the following conditions; catalytic iodine in methanol solution at room temperature ${ }^{130}$; bromine in diethyl ether ${ }^{131}$; suspension of ferric chloride adsorbed onto silica in acetone ${ }^{132}$. When treated with mineral acids such as sulfuric acid in methanol or $2 \mathrm{~N} \mathrm{HCl}$ in $\mathrm{THF} 133$ the compounds completely decomposed (sulfates are known to be hydrolysed by mineral acids). The isopropylidene groups could be selectively removed using trifluoroacetic acid in dichloromethane however better yields were obtained when ethanol was used as the solvent (see Figure 9:1).

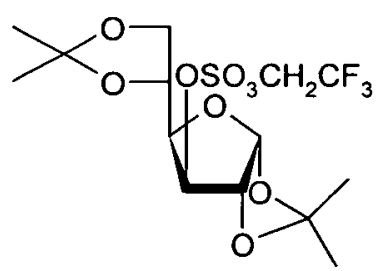

(20)

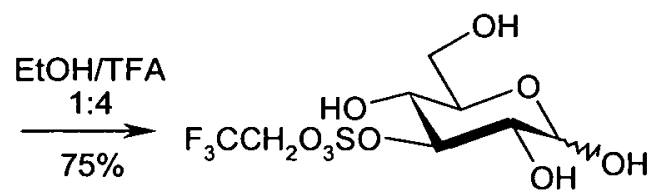

(47)

Figure 9:1: Acetal deprotection. 
In conclusion, the trifluoroethyl group is stable to strong organic acids and other mildly acidic conditions but not to mineral acids.

\subsection{Stability to Bases.}

The initial work in this project, on the sulfonates, determined that the trifluoroethyl ester of a sulfate should be stable to alkaline conditions. This was found to be true. The acetate protecting groups could be removed from 1,2,3,4-tetra- $O$-acetyl- $\beta$-Dglucopyranose 6-(2',2',2'-trifluoroethyl sulfate) (29) by methanolic sodium methoxide (see Figure 9:2). The resulting deprotected monosaccharide still contained the trifluoroethyl group (easily identified by ${ }^{19} \mathrm{~F} \mathrm{nmr}$ ).

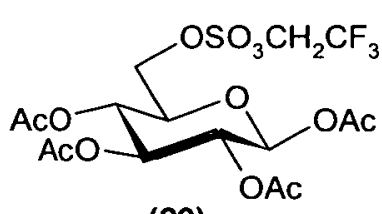

(29)

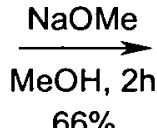

$66 \%$

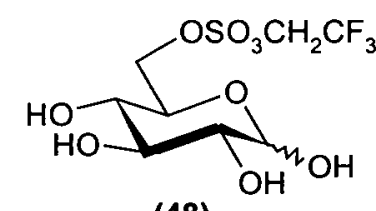

(48)

Figure 9:2: Acetate deprotection.

The trifluoroethyl protecting group is obviously stable to alkaline conditions. The low yield for this transformation may be due to loss of the trifluoroethyl group, however no sulfate monoester was recovered from the reaction.

\subsection{Stability to Hydrogenation.}

A number of commonly used protecting groups are cleaved under hydrogenation conditions, it was therefore important to show that the sulfate protecting group would not be affected by such reactions. The 4;6-benzylidene acetal of methyl 4;6-Obenzylidene- $\alpha$-D-glucoside 2-(2',2',2'-trifluoroethyl sulfate) (43) can be cleaved by standard hydrogenation conditions (Palladium on charcoal/ $\mathrm{H}_{2}$ gas/ $\mathrm{AcOH}$ ). The resulting product (49) contained the protected sulfate (see Figure 9:3). 


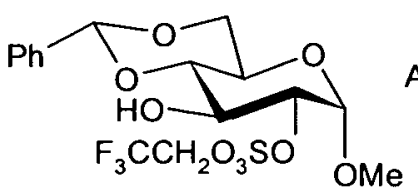

(43)

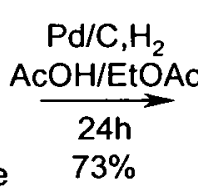

$3 \%$

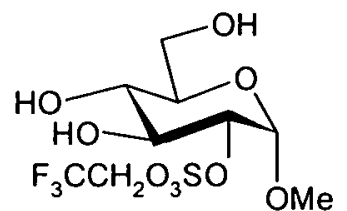

(49)

\section{Figure 9:3: Benzylidene deprotection.}

\subsection{Fluoride Stability.}

Silyl protecting groups are occasionally used in carbohydrate chemistry and are primarily removed by exposure to fluoride ions. $1 ; 2,3 ; 4$-di- $O$-isopropylidene- $\alpha$-Dgalactopyranose 6-(2',2',2'-trifluoroethyl sulfate) (30) was totally stable to tetrabutyl ammonium fluoride in acetone and to tetra-butyl ammonium iodide. There was no trace of any deprotected products and no sign of any products from the displacement of the protected sulfate by halide ions (see Figure 9:4).

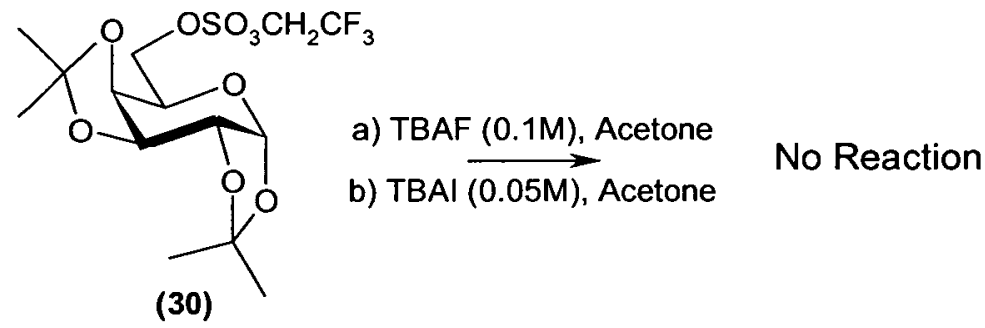

Figure 9:4: Halide ion stability.

\subsection{Conclusions.}

This section has shown that the trifluoroethyl group is a highly stable protecting group for sulfate monoesters and that it is not affected by common reaction conditions. It is stable to strong organic acids and hydrogenation conditions. It is unaffected by alkalis or other nucleophiles. Obviously it is not possible to test the groups stability against all reaction conditions but this section details a representative group of examples. The following sections include a larger number of further examples where the protected sulfate is carried through a number of reaction steps and is exposed to a variety of reagents and conditions. 
A short communication describing the synthesis and stability of these protected monosaccharide sulfates has been published ${ }^{134}$ (See Appendix 1).

\section{Utility: Use in synthesis.}

This section explores further the stability of protected sulfates. If the sulfate ester protecting group is to be of practical use in synthesis it must be stable to conditions used to assemble oligosaccharides from their component monosaccharides. These glycosidation reactions can be performed under an almost infinite variety of conditions and it is beyond the scope of this work to examine the stability of the trifluoroethyl sulfate ester to them all. However some of the more widely accepted and used procedures have been examined.

This section describes a number of attempts to form a disaccharide containing a protected sulfate. It also establishes a few basic rules and strategies for the use of protected sulfates in the synthesis of larger oligosaccharides.

Although their have been a large number of glycosidation reactions published most are based on the principle of a glycosyl donor reacting with a glycosyl acceptor in the presence of a glycosyl promoter (see Figure 10:1). The diversity arises from the various glycosyl donor leaving groups $(\mathrm{X})$, the different glycosyl promoters and the general reaction conditions 135 .
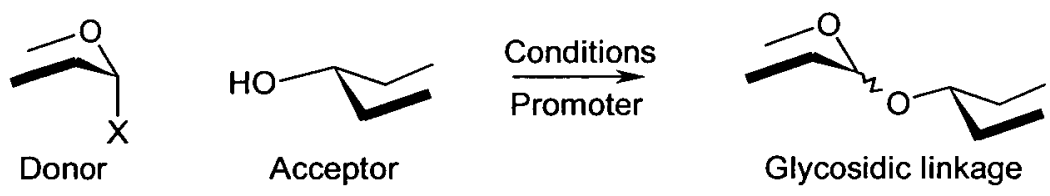

Figure 10:1: Glycosidation reaction.

This section has been divided in to two parts each discusses the preparation and use of either glycosyl donors or glycosyl acceptors. 


\subsection{Glycosyl Donors.}

Any attempt to prepare and examine all of the published types of glycosyl donors would be totally unfeasible, only a small number of representative examples have been examined.

Thioglycosides are some of the most widely used glycosyl donors ${ }^{136}$, they can be activated by a number of promoters and have the added advantage of being easily oxidised to the glycosyl sulfoxide which have even greater reactivity ${ }^{137}$.

\subsubsection{Activation of glycoside donor.}

Thioglycosides are often conveniently prepared by conversion of the corresponding anomeric acetate. The glycosyl acetate is mixed with a thiol (usually ethanethiol or thiophenol) in the presence of a Lewis acid. Compounds (29) and (32) had been prepared previously, both have anomeric acetates and were good substrates on which to test this approach to thio glycosides.

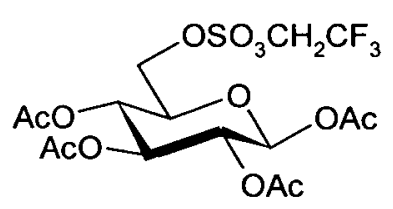

(29)

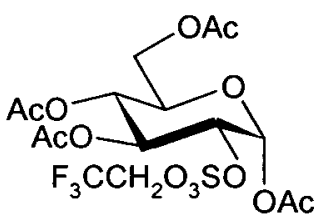

(32)

Figure 10:2: Glycosyl Acetates.

Compound (32) was reacted under a variety of conditions known to convert anomeric acetates to thioglycosides. Results are shown in the table below:-

Table 10:4: Conversion of acetate to thioethyl glycoside.

\begin{tabular}{|c|c|c|c|}
\hline Lewis Acid & Conditions & Time & Result \\
\hline $\mathrm{ZrCl}_{4}{ }^{138}$ & $0^{\circ} \mathrm{C}, \mathrm{DCM}, \mathrm{EtSH}$ & $6 \mathrm{~h}$ & No reaction \\
\hline $\mathrm{ZnCl}_{2}{ }^{139}$ & $30^{\circ} \mathrm{C}, \mathrm{DCM}, \mathrm{EtSH}$ & $6 \mathrm{~h}$ & No reaction \\
\hline TMS Triflate 140 & $0^{\circ} \mathrm{C}, \mathrm{DCM}, \mathrm{EtSH}$ & $6 \mathrm{~h}$ & $\begin{array}{c}\text { Anomerised starting } \\
\text { material }\end{array}$ \\
\hline $\mathrm{ZrCl}_{4}{ }^{138}$ & $25^{\circ} \mathrm{C}, \mathrm{MeOH}, \mathrm{EtSH}$ & $2 \mathrm{~h}$ & Decomposition \\
\hline
\end{tabular}


A trial reaction was set up where $1,2,3,4,6$-penta- $O$-acetyl- $\alpha$-D-glucopyranose (50) was treated with ethanethiol in the presence of zirconium tetrachloride. The thioglycoside was formed very rapidly. Obviously the presence of the bulky and electron withdrawing sulfate at the 2-position hinders the reaction.

Compound (29) was treated with thiophenol in the presence of some different Lewis acids and the results are shown in the table below.

Table 10:5: Conversion of acetate to thiophenyl glycoside.

\begin{tabular}{|c|c|c|c|}
\hline Lewis Acid & Conditions & Time & Result \\
\hline $\mathrm{SnCl}_{4}^{138}$ & $25^{\circ} \mathrm{C}, \mathrm{DCM}, \mathrm{PhSH}$ & $24 \mathrm{~h}$ & Decomposition \\
\hline $\mathrm{BF}_{3}^{141}$ & $25^{\circ} \mathrm{C}, \mathrm{DCM}, \mathrm{PhSH}$ & $24 \mathrm{~h}$ & Decomposition \\
\hline TMS Triflate ${ }^{140}$ & $25^{\circ} \mathrm{C}, \mathrm{DCM}, \mathrm{PhSH}$ & $24 \mathrm{~h}$ & Decomposition \\
\hline $\mathrm{ZnCl}_{2}{ }^{139}$ & $25^{\circ} \mathrm{C}, \mathrm{DCM}, \mathrm{PhSH}$ & $24 \mathrm{~h}$ & No reaction \\
\hline $\mathrm{HgCl}_{2}$ & $25^{\circ} \mathrm{C}, \mathrm{DCM}, \mathrm{PhSH}$ & $24 h$ & No reaction \\
\hline $\mathrm{FeCl}_{3}{ }^{142}$ & $25^{\circ} \mathrm{C}, \mathrm{DCM}, \mathrm{PhSH}$ & $24 \mathrm{~h}$ & Decomposition \\
\hline $\mathrm{ZrCl}_{4}{ }^{138}$ & $0^{\circ} \mathrm{C}, \mathrm{DCM}, \mathrm{PhSH}$ & $1 \mathrm{~h}$ & No reaction \\
\hline $\mathrm{ZnCl}_{2}{ }^{139}$ & $0^{\circ} \mathrm{C}, \mathrm{DCM}, \mathrm{PhSH}$ & $1 \mathrm{~h}$ & No reaction \\
\hline $\mathrm{ZrCl}_{4}{ }^{138}$ & Reflux, $\mathrm{CH}_{3} \mathrm{CN}, \mathrm{PhSH}$ & $6 \mathrm{~h}$ & No reaction \\
\hline $\mathrm{ZnCl}_{2} 139$ & Reflux, $\mathrm{CH}_{3} \mathrm{CN}, \mathrm{PhSH}$ & $6 \mathrm{~h}$ & No reaction \\
\hline $\mathrm{SnCl}_{4}^{138}$ & $0^{\circ} \mathrm{C}, \mathrm{DCM}, \mathrm{PhSH}$ & $15 \mathrm{~min}$ & Some reaction \\
\hline $\mathrm{ZnCl}_{2}^{139}$ & Reflux, DCM, PhSH & $96 \mathrm{~h}$ & $\begin{array}{c}\text { Some reaction } \\
\text { (See Figure 10:3) }\end{array}$ \\
\hline
\end{tabular}

When (29) was refluxed with thiophenol and zinc chloride in dichloromethane for a prolonged period of time a reaction was observed however the product was found not to be the desired compound but the di-thiophenol substituted compound (51) (see Figure 10:3). 


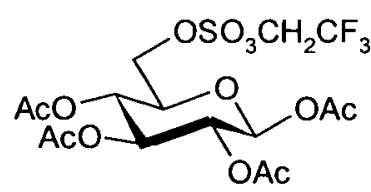

(29)

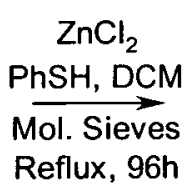

Reflux, 96h

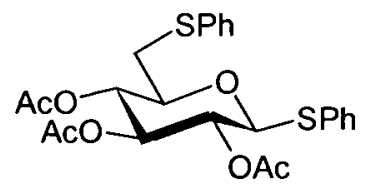

(51)

Figure 10:3: Nucleophilic displacement by thiophenol.

The trifluoroethyl protected sulfates must be able to act as a leaving group. A property that would be enhanced by the presence of the Lewis acid. Given the similarity between the trifluoroethyl protected sulfates and trifluoromethyl sulfonates which are known to be good leaving groups this result was to be expected.

An observation that was made was that addition of the Lewis acid to the sulfated sugar in solution often led to formation of a precipitate. It is possible that the Lewis acid binds to the sulfated sugar forming an insoluble or otherwise unreactive complex. It is possible to draw another canonical form of the sulfate diester (see Figure 10:4) which demonstrates that the protected sulfate could preferentially bind the Lewis acid making it unavailable for activating the anomeric acetate.

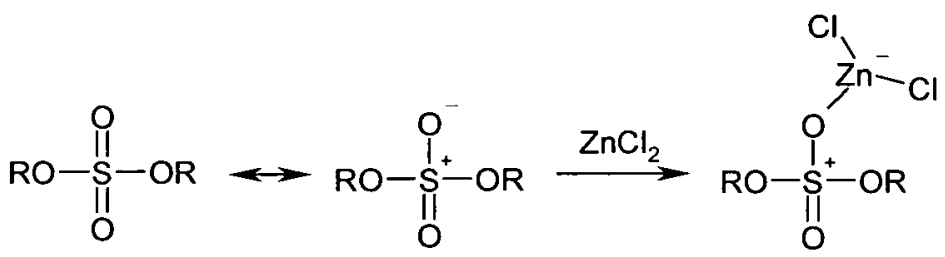

Figure 10:4: Canonical form of sulfate.

Anomeric acetates are versatile intermediates in carbohydrate chemistry and can be converted to other activated donors other than thio glycosides. Glycosyl bromides are easily prepared from the acetate. Although not very stable they have been used extensively in carbohydrate synthesis in the well known Koenigs-Knorr glycosidation reaction ${ }^{143}$. Compound (32) was treated with phosphorous tribromide under conditions known to convert the acetate to the anomeric bromide ${ }^{144}$ which may then be used in glycosidation reactions (see Figure 10:5). However no reaction was observed even after a prolonged period of time. The conventional method of converting anomeric acetates to bromides using hydrogen bromide in acetic acid or 
acetic anhydride was not attempted due to the intolerance of protected sulfates to strong mineral acids.

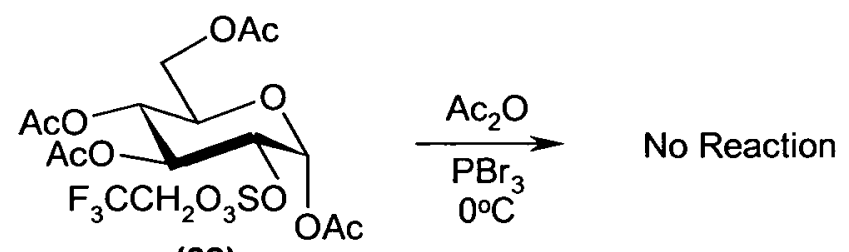

(32)

Figure 10:5: Conversion to Glycosyl Bromide.

This lack of reactivity could again be attributed to the steric bulk or electron withdrawing ability of the 2-sulfate di ester (32).

Another stable activated glycosyl donor accessible from the anomeric acetate is the trichloroacetimidate, the use of which has been pioneered by Schmidt ${ }^{145}$. To obtain the trichloroacetimidate the anomeric position must be deprotected selectively and the resulting alcohol reacted with trichloroacetonitrile in the presence of base (see Figure 10:6).

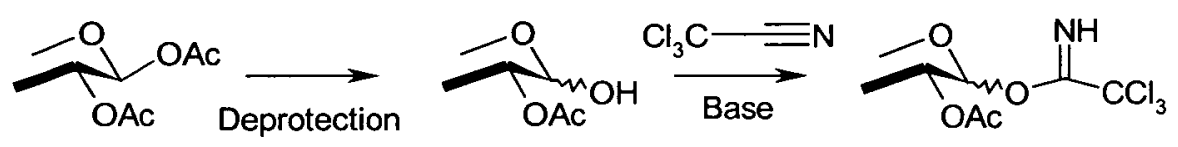

Figure 10:6: Trichloroacetimidate.

The anomeric acetate is more reactive than the other acetates on a peracetylated sugar ring. This means that it maybe removed selectively without difficulty. Although the anomeric acetate of (32) could be removed in good yield to give the alcohol ${ }^{146}$ (52) it was not observed to react with trichloroacetonitrile (see Figure 10:7) upon addition of potassium bicarbonate ${ }^{147}$.

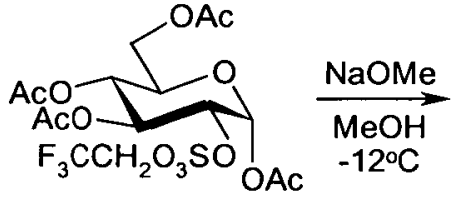

(32)

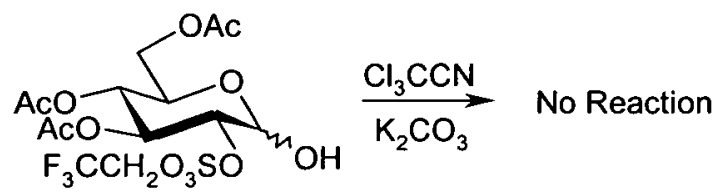

(52)

Figure 10:7: Selective deprotection. 
Once again the 2-sulfate's electron-withdrawing effect will inhibit the reaction by decreasing the nucleophilicity of (52). Also the presence of the bulky trifluoroethyl sulfate at the 2-position will interfere with the approach of (52) to trichloroacetonitrile.

When the anomeric acetate of (29) was removed using mildly basic conditions the isolated product was not the expected alcohol but the 1,6-anhydro compound (53) the data for which were identical to that published for the known compound ${ }^{148}$ (see Figure 10:8). This another example of the ability of the trifluoroethyl sulfate ester to act as a good leaving group.

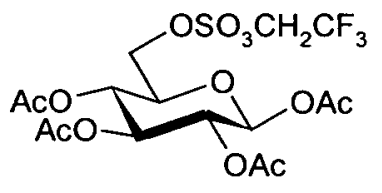

(29)

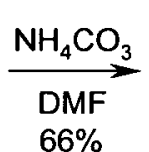

$66 \%$

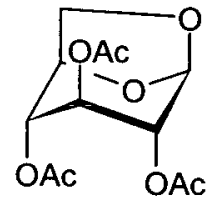

(53)

Figure 10:8: 1,6-Anhydro compound.

The published synthesis of this compound involves treating 1,2,3,4-tetra-O-acetyl-6(para-toluene sulfonyl)- $\beta$-D-glucopyranose with base. It is interesting to note that the yield for the conversion of (29) to (53) $(66 \%)$ was greater than the conversion of the 6- para-toluene sulfonyl to the anhydro compound 148 which gave a yield of $(58 \%)$, it could therefore be argued that the protected sulfates are as good if not better leaving groups than para-toluene sulfonyl groups. This is a point that probably deserves further examination.

\subsubsection{Pre-activation of protected sulfates.}

The problems encountered during this preliminary work establish one simple ground rule for use of protected sulfates in synthesis which is that the monosaccharide unit should be activated before the protected sulfate is introduced.

With this in mind the synthesis of two alternative donors (54) and (55) were designed. The synthesis of both compounds again makes extensive use of the 
differences in reactivity of the various ring positions and the special relationship between the anomeric centre and the protecting group at the 2-position.

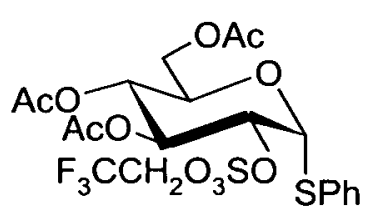

(54)

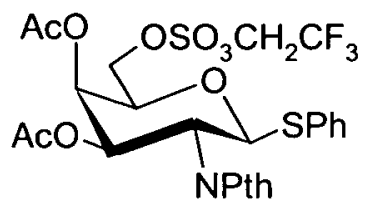

(55)

Figure 10:9: Glycosyl donors.

Treating 1,2,3,4,6-penta- $O$-acetyl- $\beta$-D-glucopyranose (50) with phosphorous penta chloride gives the 2-trichloroacetyl glycosyl chloride ${ }^{149}$ (56) probably via an electrophilic mechanism ${ }^{150}$ (see Figure 10:10).

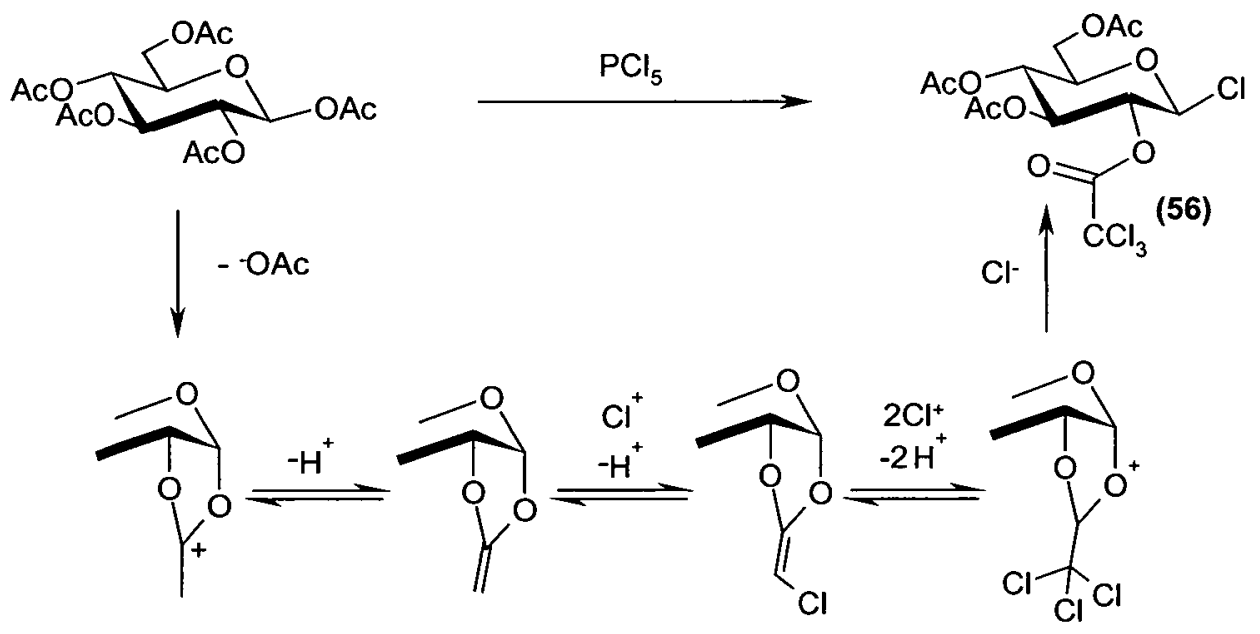

Figure 10:10: Electrophilic chlorination.

The 2-trichloroacetyl group of (56) can be removed selectively with ammonia in ether at low temperature to give (57) ${ }^{151}$ the chloride can then be converted to the thio ether ${ }^{152}$ (58) and the free 2-position sulfated and protected in good yield to give (54) (see Figure 10:11). 


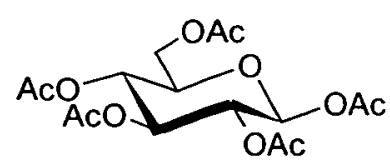

(50)

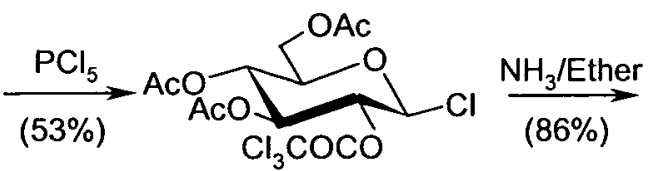

(56)

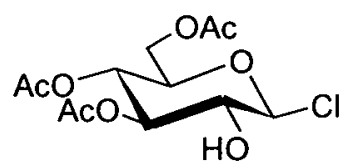

(57)

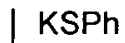

$(40 \%)$

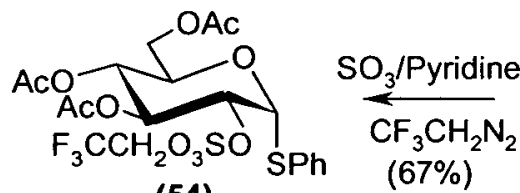

(54)
$(67 \%)$

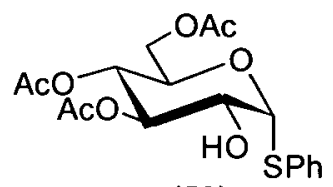

(58)

Figure 10:11: Synthesis of (54).

The structure of (54) was further confirmed by X-ray crystallography see Figure 10:12 and Appendix 2 for structure and data. Notably this is only the third report of an X-ray crystal structure of a di-alkyl sulfate diester ${ }^{153-155}$.

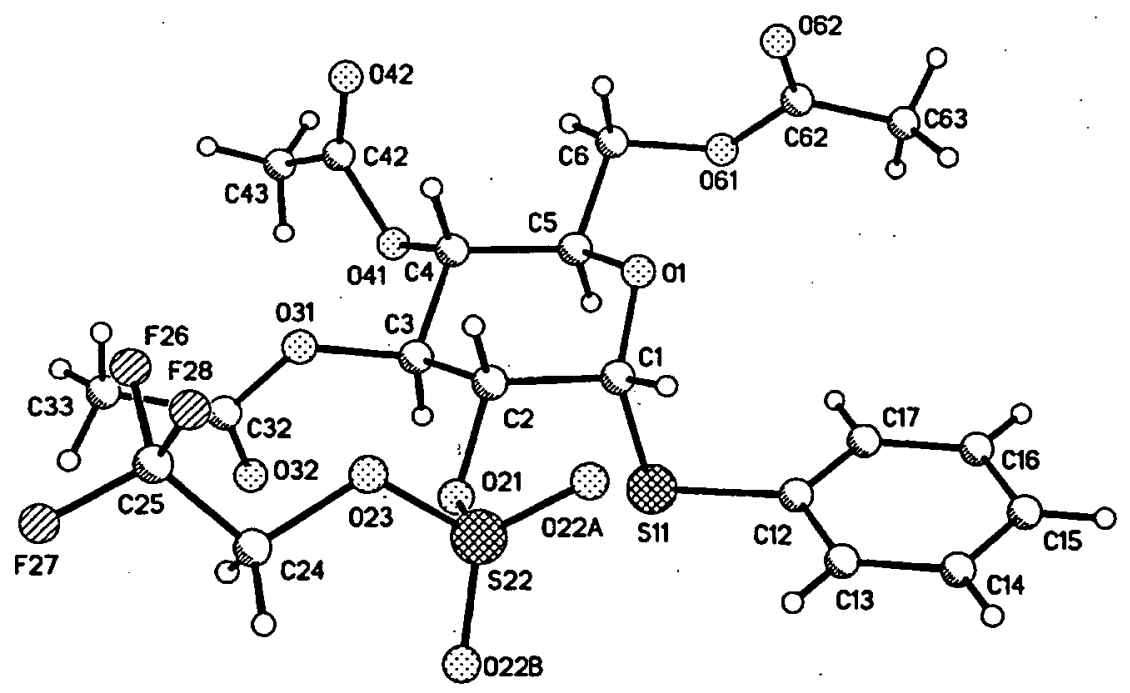

Figure 10:12: X-ray structure of (54).

Attempts were made to react (54) with ethanol under a number of conditions commonly used to promote glycosidation reactions ${ }^{156}$; the results are shown in Table 10:6:- 
Table 10:6

\begin{tabular}{|c|c|c|c|}
\hline Donor & Acceptor & Conditions & Result \\
\hline $\mathbf{( 5 4 )}$ & EtOH & NBS, $\mathrm{DCM}^{156}$, reflux & No reaction \\
\hline $\mathbf{( 5 4 )}$ & $\mathrm{EtOH}$ & $\mathrm{NBS}, \mathrm{TfOH}^{157}, \mathrm{DCM}, 25^{\circ} \mathrm{C}$ & No reaction \\
\hline $\mathbf{( 5 4 )}$ & $\mathrm{EtOH}$ & $\mathrm{NIS}, \mathrm{TfOH}^{158}, \mathrm{DCM}, 25^{\circ} \mathrm{C}$ & No reaction \\
\hline
\end{tabular}

These reactions would seem to illustrate that the protected sulfate acts as a powerful "disarming" group. However the lack of reactivity could also be explained by the sulfate interfering with the $\mathrm{Br}^{+} / \mathrm{I}^{+}$ions formed thus preventing the reaction.

Given (54)'s lack of reactivity the thio ether was oxidised with meta-chloro perbenzoic acid to the glycosyl sulfoxide (59) (see Figure 10:13). The sulfoxides have been reported by Kahne ${ }^{159}$ to be significantly more reactive than their parent thio glycosides.

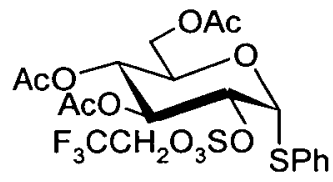

(54)

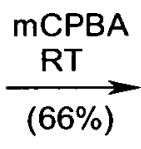

$66 \%)$

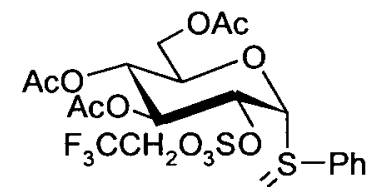

(59)

\section{Figure 10:13: Oxidation of thioglycoside.}

When (59) was reacted with 1,2,3,4-tetra- $O$-acetyl- $\beta$-D-glucopyranose (13) under conditions used to perform glycosidations on sulfoxides ${ }^{160}$ one new product was visible by TLC, direct injection of the crude reaction mixture into an mass spectrometer using electrospray ionisation indicated peaks at the mass expected for the disaccharide (60) (see Figure 10:14). 

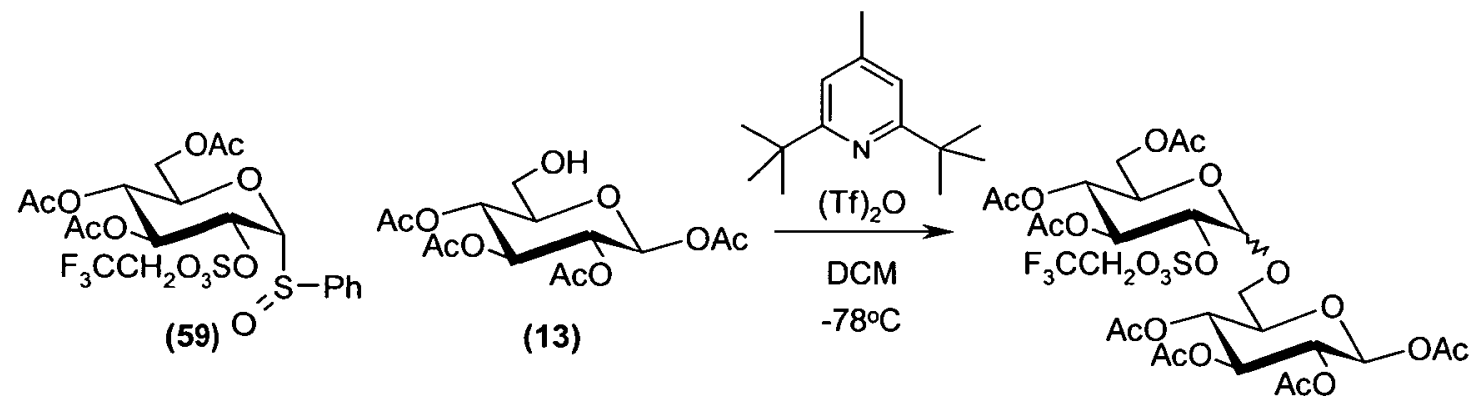

(60)

Figure 10:14: Attempted synthesis of disaccharide.

The reaction was worked up in the usual manner and the product was isolated by column chromatography; however it was identified not as the desired disaccharide but was found to be 1,2 anhydro compound (61) which is also known as Birgl's anhydride ${ }^{161}$ (see Figure 10:15).

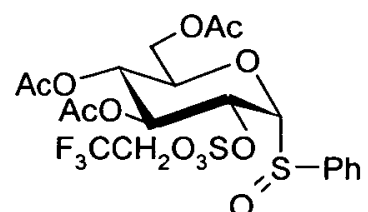

(59)

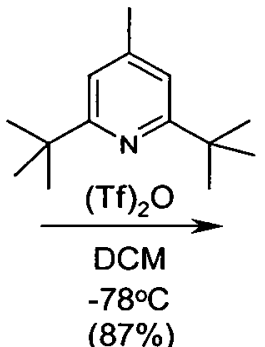

$(87 \%)$

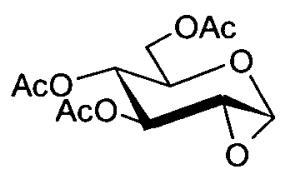

(61)

Figure 10:15: Birgl's anhydride.

The intended glycosidation reaction the triflic anhydride activates the sulfoxide and a sulfinate ester is expelled (see Figure 10:16) the resulting oxycarbenium cation is then captured by the triflic acid produced to form a triflate which is subsequently displaced by the nucleophile ${ }^{160}$. However in this case the triflic acid formed might be a sufficiently powerful nucleophile to attack the protected sulfate. The resulting alkoxide closes on the carbenium cation to form the anhydride (see Figure 10:16). 


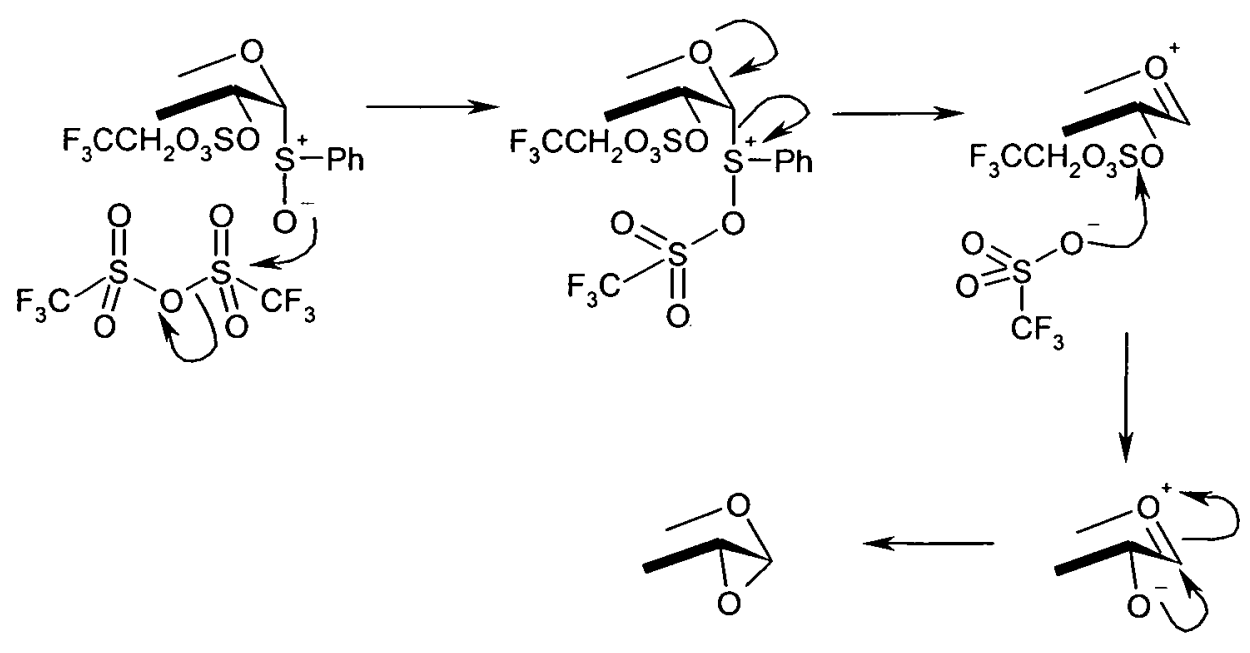

Figure 10:16: Mechanism for anhydride formation.

It would seem that even with the enhanced reactivity of the sulfoxide only trace amounts of the desired products are formed.

To avoid any difficulties due to the influence of the protected sulfate being at the 2position the 6-sulfate (55) was examined. The $N$-phthalimido thio glycoside (62) was available from previous work done in our research group in which it had been used successfully in glycosyl coupling reactions. It is possible to exploit the higher reactivity of the primary alcohol in the 6-position to selectively alkylate the 6hydroxyl of (62) with a trityl (triphenylmethyl) group ${ }^{162}$ to give (63).

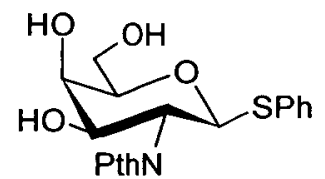

(62)

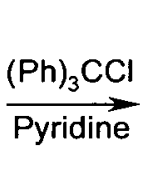

ne

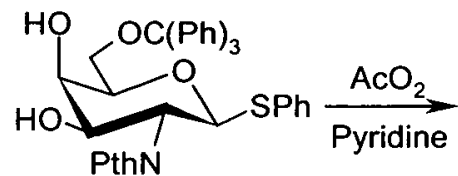

(63)

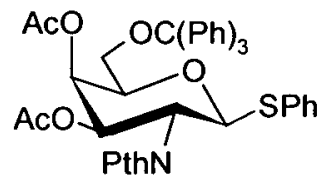

(64)

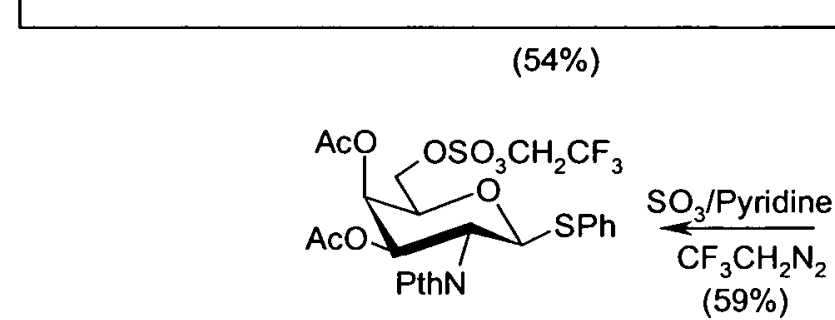

(55)
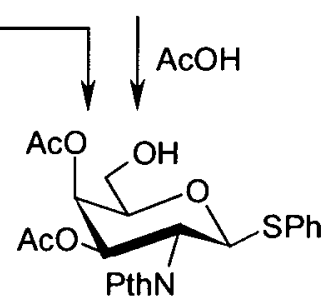

(65)

Figure 10:17: Synthesis of (55). 
The 3 and 4-positions of (63) could then be acetylated in the usual manner to give (64), hydrolysis of the trityl group with acetic acid gave (65) which was subsequently sulfated and protected to give (55) (see Figure 10:17) in good overall yield.

Two glycosidic coupling reactions have been attempted with (55) as the glycosyl donor. With 1,2,3,4-tetra- $O$-acetyl glucopyranose as the acceptor the isolated product was not the desired disaccharide but the previously identified compound (53) (see Figure 10:18). The iodonium ions generated must activate the anomeric centre to displacement by the 6-hydroxyl group.

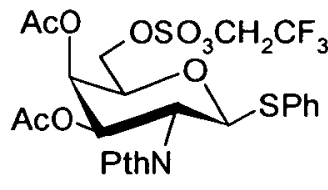

(55)

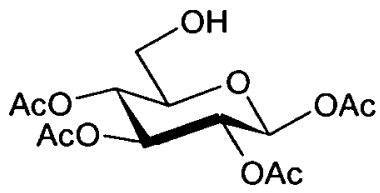

(13)
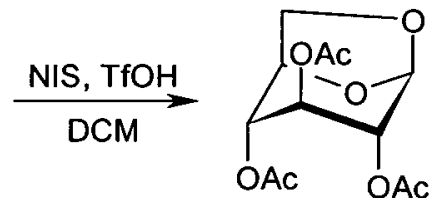

(53)

Figure 10:18: Failed glycosidic coupling.

When 1;2,3;4-di-O-isopropylidene galactopyranose (12) was used as the acceptor no reaction was observed by TLC; however a quantity of the product was observed by electrospray MS (see Figure 10:19). Fractions containing the disaccharide were isolated by column chromatography but were found to contain at least one other unidentified compound and the disaccharide could not be properly characterised.

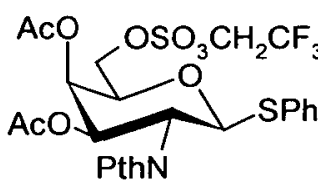

(55)

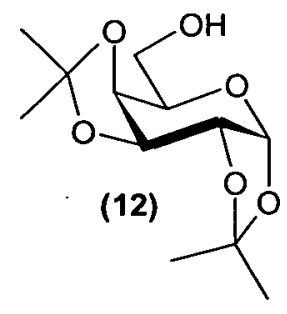

$\underset{\mathrm{NCM}}{\stackrel{\mathrm{NIS}, \mathrm{TfOH}}{\longrightarrow}}$

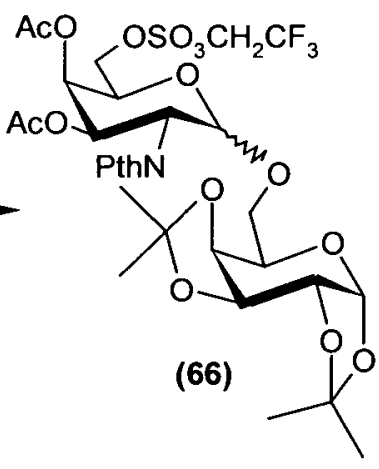

Figure 10:19: Synthesis of (66).

Given that traces of product can be identified in the above reaction it maybe that the more reactive sulfoxide would generate an isolatable quantity of the disaccharide, however this work has not been attempted due to a lack of material and time. 
This section of the project has failed to produce a protected sulfated donor that can be used in oligosaccharide synthesis but it has produced evidence that suggests that such targets are not impossible. As is often the case in carbohydrate chemistry a particular linkage must be examined as an individual case and the appropriate conditions developed for that precise situation. Further work is required to further develop this area.

\subsection{Glycosyl Acceptors.}

As with the glycosyl donors there are an almost infinite number of potential sulfated glycosyl acceptors. This work describes the synthesis and attempted synthesis of some protected sulfated monosaccharide acceptors and their use in coupling reactions. Where possible the acceptors have been chosen to be similar to published glycosidations so that there can be a direct comparison between the sulfated and non sulfated cases.

As described in the introductory chapter there has been a great deal of recent interest in Alfalfa Nod factors and related molecules. As mentioned one possible strategy to their synthesis would be to start with a sulfated acceptor at the reducing end of the molecule and extend the molecule in the other direction. To do this a compound like (67) would be required (see Figure 10:20). Where $\mathrm{P}$ and $\mathrm{P}$ ' are orthogonal protecting groups.

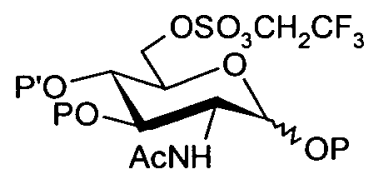

(67)

\section{Figure 10:20: Nod Factor acceptor.}

A strategy to a compound of this type was developed, based on the work of Johansson and Samuelsson. They have described the synthesis of (68) via the 
reductive ring opening of the para-methoxy benzylidene ring of (69) ${ }^{163}$ (see Figure $10: 21)$

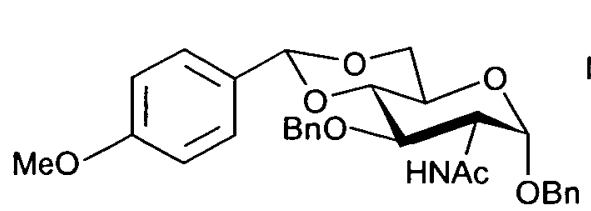

(69)

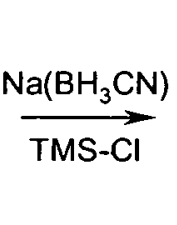

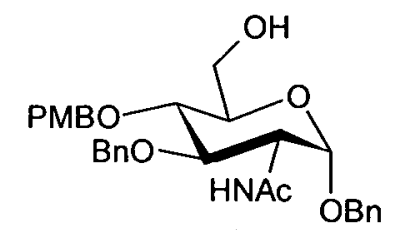

(68)

Figure 10:21: Reductive ring opening.

The 6-position is then open to sulfation and the para-methoxybenzyl group can be selectively cleaved with cerium (IV) ammonium nitrate (CAN) allowing extension of the sugar chain on the 4-position.

Compound (69) is made starting from $N$-acetyl glucosamine (73). The free glucosamine was converted to the benzyl glycoside (70) by a standard Fisher glycosidation reaction ${ }^{164}$. The para-methoxybenzylidene (71) ring was then created by stirring a mixture of (70) and 4-methoxybenzaldehyde dimethyl acetal under a slight vacuum such that the methanol formed was evaporated ${ }^{163}$. The resulting benzylidene acetal (72) was obtained in reasonable purity without the need for column chromatography. After some initial difficulties it was possible to benzylate the 3-position of (72) to give (69) (see Figure 10:22).

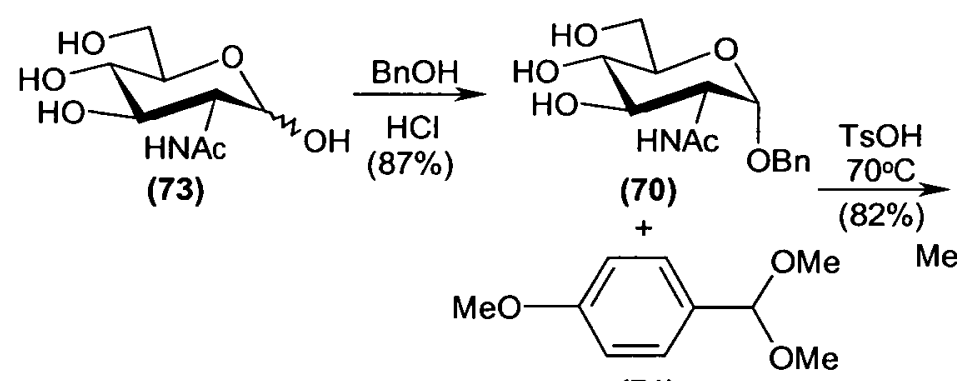

(71)
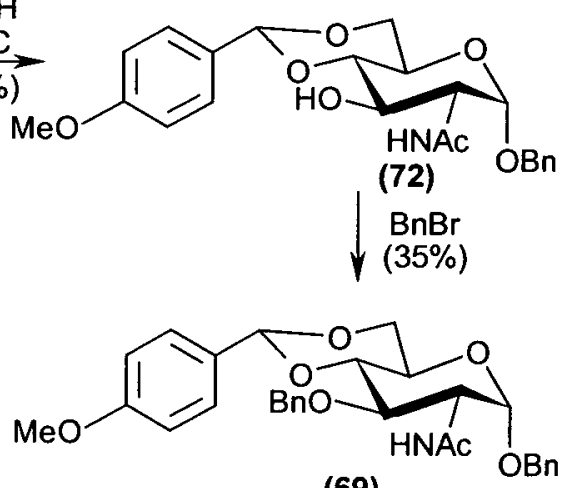

(69)

Figure 10:22: Synthesis of (69). 
The published conditions ${ }^{163}$ for the benzylation of (72) had to be modified to prevent the para-methoxy benzylidene ring from being hydrolysed by the hydrogen bromide liberated in the reaction.

The para-methoxybenzylidene ring can be opened in two ways, such that the 6position is left free or such that the 4-position is left free. The regioselectivity of the reaction is determined by allowing the reaction to proceed under either thermodynamic control ${ }^{165}$ (gives O-6 benzyl derivative) or kinetic control ${ }^{166}$ (gives O-4 benzyl derivative) and this is largely determined by the size of the electrophile used in the reaction. Johansson and Samuelsson reductively open the methoxybenzylidene acetal with sodium cyanoborohydride in the presence of trimethylsilyl chloride (which acts as the electrophile in the reaction.) As trimethylsilyl is a bulky group it cannot access the 4-position due to the steric hindrance of the benzyl group at the 3-position it therefore binds to the 6-position so directing the outcome of the reductive cleavage (see Figure 10:23) i.e. the reaction proceeds under kinetic control.

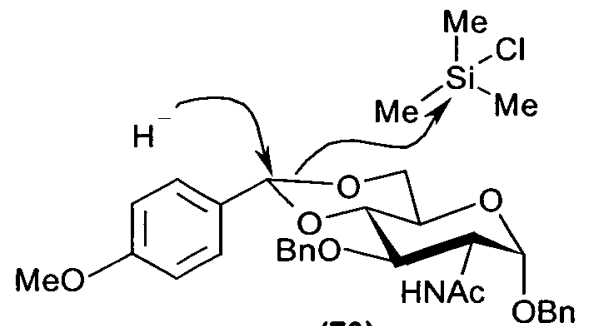

(70)

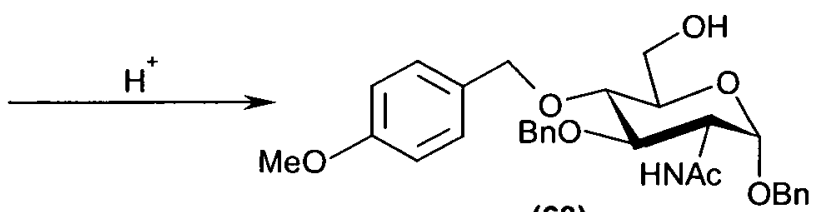

(68)

Figure 10:23: Kinetic control.

The reaction did not proceed as describe in the literature, the methoxybenzylidene acetal (69) was not soluble in the stated solvent (acetonitrile) so the reaction was performed in dichloromethane. The result was a 3:2 mixture of the 6-paramethoxybenzyl (74) over the 4- para-methoxybenzyl (68) (i.e. the wrong isomer was in abundance) and an extremely low yield ( $8 \%)$ see Figure 10:24. 


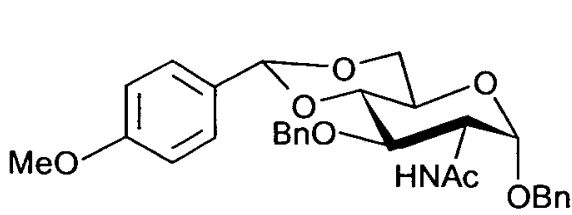

(69)

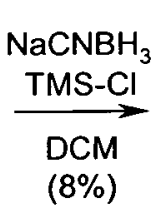

$(8 \%)$

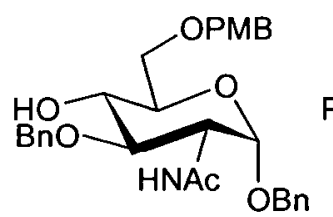

(74)

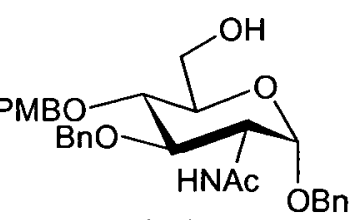

(68)

$3: 2$

Figure 10:24: Reductive ring cleavage.

Although the result of this reaction was wholly unsatisfactory, it was believed that the problems with this step could be overcome. (68) was sulfated under the standard conditions with sulfur trioxide pyridine complex. The reaction was found to give a mixture of products even when reacted at room temperature. Apparently the paramethoxybenzyl group is too acid sensitive to be stable to the sulfation conditions employed. This is a major flaw in the strategy for the synthesis of these compounds. The problem maybe overcome in one of two ways.

The para-methoxybenzylidene acetal could be replaced by a benzylidene acetal that can be opened selectively in a similar fashion but the resulting benzyl group ${ }^{165}$ is more stable to acid. However this would necessitate a change in the protection of the other ring positions, if the 4-position is still to be accessible.

The other possibility is to open the acetal thermodynamically onto the 6-position, protect the 4-position with another orthogonal, acid stable protecting group (such as acetate) then removal of the para-methoxybenzyl group from the 6-position allowing sulfation. Although likely to work this last approach is rather long winded and has not been attempted.

The purpose of this part of the project is not to synthesis Alfalfa Nod factor but to produce a sulfated glycosyl acceptor for examination under glycosidation conditions. Therefore this part of the project has not been continued.

Allyl $N$-acetyl-2-deoxy-2,3-di- $O$-acetyl-glucosamine (75) can be easily made from $\mathrm{N}$-acetyl glucosamine using a similar approach to that of (65) and was available from 
previous work done within the research group ${ }^{167}$. Sulfation and protection gave (76) in reasonable yield. The $O$-acetyl groups can be removed giving simple access to a potential glycosyl acceptor (77) (see Figure 10:25).

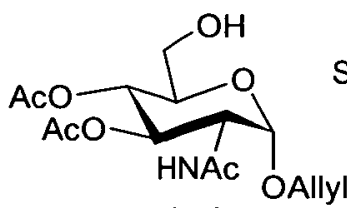

(75)

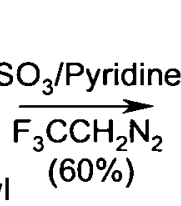

$0 \%$ )

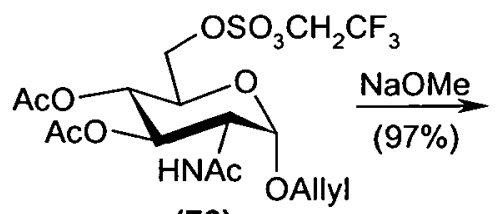

(76)

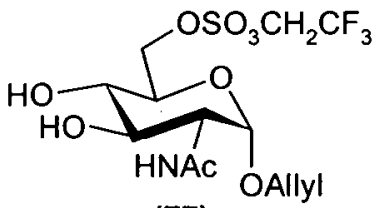

(77)

Figure 10:25: Synthesis of (77).

An attempt was made to glycosidate compound (77) with a thiophenyl donor (78) (available from the acetylation of (62)) under commonly used conditions. However no reaction was observed (see Figure 10:26).

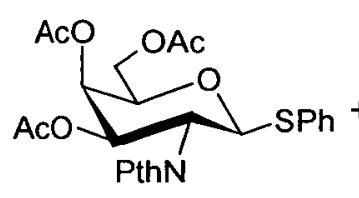

(78)

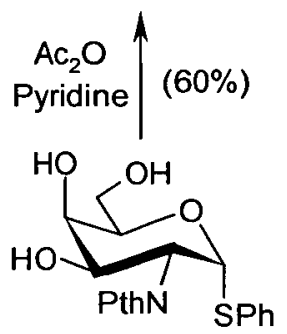

(62)

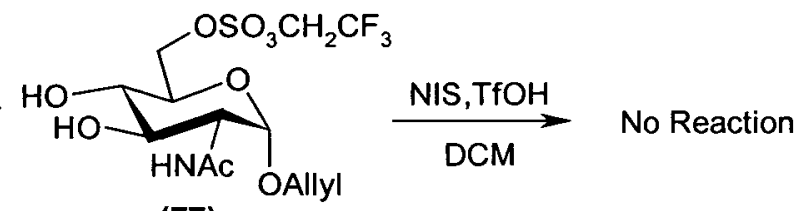

(77)

Figure 10:26: Failed glycosidation.

Matta et al. ${ }^{168}$ have reported the regioselective glycosidation of a related compound (79) by a galactosyl bromide (80) under the Friedlich conditions of a refluxing mixture of benzene and nitromethane in the presence of mercuric cyanide (see Figure 10:27). 


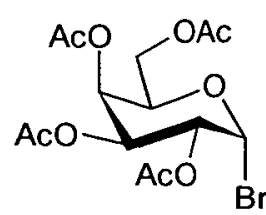

(80)

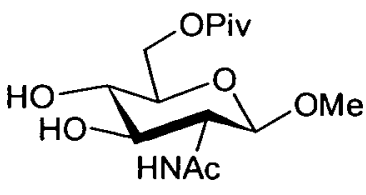

(79)<smiles>CO[N+](=O)[O-]</smiles>

Ac

$\mathrm{AcO}$

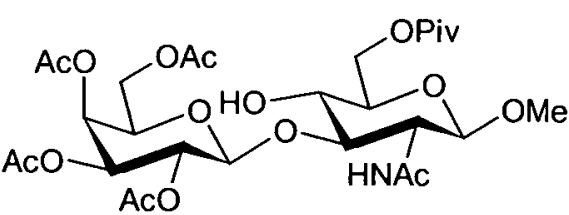

(81)

Figure 10:27: Friedlich conditions.

When compound (77) was reacted with 2,3,4,6-tetra- $O$-acetyl- $\alpha$-D-glucopyranosyl bromide (82) in the presence of mercuric cyanide under similar conditions to above but over a prolonged period of time a poor yield (21\%) of the disaccharide (83) was obtained (see Figure 10:28).

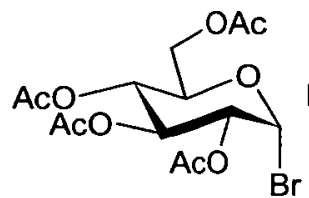

(82)

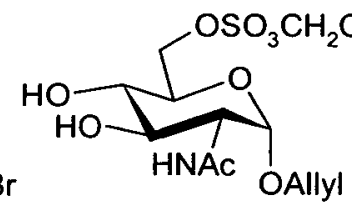

(77)

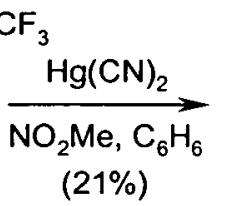

(21\%)

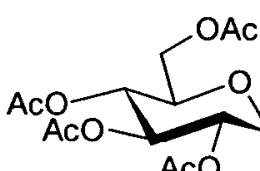

(83)

Figure 10:28: Synthesis of (83).

The reaction conditions remove the allyl protecting group from the reducing end of the disaccharide. The anomeric configuration was assigned as $\beta$ by comparison to literature examples and by examination of the ${ }^{13} \mathrm{C}-\mathrm{NMR}$ compound.

\subsection{Conclusions.}

A number of preliminary experiments have shown that protected sulfates may be incorporated into glycosyl donors or acceptors that can be used in synthesis. A number of "ground rules" for the use of a protected sulfate in a molecule have been established.

\section{Glycosyl Donors:}

The protected sulfate should be introduced into the monosaccharide unit after the anomeric centre has been converted to its donor functionality. 
The presence of the protected sulfate at the 2-position of a glycosyl donor interferes with glycosidation reactions. Whether this is due to its deactivating nature or simply because it complexes to the activating agent has not been determined.

\section{Glycosyl Acceptors:}

The protected sulfate can be incorporated into glycosyl acceptors that can successfully be used in glycosidation reactions.

The work done highlights that the inclusion of a protected sulfate is not straight forward and that problems may arise when Lewis acids are used in the synthesis. This however is not a significant problem. Despite significant improvements in methods for the synthesis of a glycosidic linkage no single all encompassing method has yet emerged. A particular connection between two monosaccharide units remains an independent challenge requiring experimental validation in which the restrictions imposed by the sulfate protecting group can be considered.

\section{Deprotection of Protected Sulfate Diesters.}

The previous sections have comprehensively demonstrated that the trifluoroethyl sulfates are simple to introduce and are highly stable. This section now examines the deprotection of the sulfates and explores some methods for the removal of the trifluoroethyl group.

There are three main considerations regarding the deprotection step that will be discussed in this section; reliability; the "harshness" of the conditions required and the compatibility of other functionality and protecting groups.

Obviously both the synthesis and stability of a protecting group are essential areas to explore however a more important issue is the removal of the protecting group. Once a synthesis has reached the deprotection stage it has involved a substantial 
commitment of time and resources therefore the deprotection step must be totally dependable if failure is to be avoided. Consequently the way in which the deprotection step is performed is less important than its reliability. A synthesis can be designed to take into account a harsh set of deprotection conditions if those conditions are fully trustworthy.

The third consideration is "orthogonality" or compatibility of the removal method with other protecting groups and the functionality of the parent molecule. The previous sections have shown that the trifluoroethyl group is orthogonal to the manipulations of many common protecting groups this section will discuss briefly how orthogonal other protecting groups are to the deprotection protocol of the trifluoroethyl group. As the deprotection of the sulfate esters would ideally be performed as the last step in a synthesis due to the cumbersome physical properties of the sulfate ester the question of its orthogonality is relatively unimportant. The trifluoroethyl deprotection method does not need to be compatible with any other protecting group only the backbone of the parent sugar.

\subsection{Deprotection using Sodium Methoxide.}

The initial work done on this project suggested that the trifluoroethyl group could be removed from para-toluenesulfonyl esters with sodium methoxide. It has already been shown that the trifluoroethyl group is stable to methoxide for the short periods of time required to remove acetates.

Compound (20) was stirred at room temperature in a solution of sodium methoxide in methanol for $16 \mathrm{~h}$. No product sulfate could be observed. The mixture was then heated to reflux, the reaction was then observed to proceed slowly and was complete after $48 \mathrm{~h}$ (see Figure 11:1) the sodium salt of the desired sulfate (84) was obtained in almost quantitative yield (99\%). 

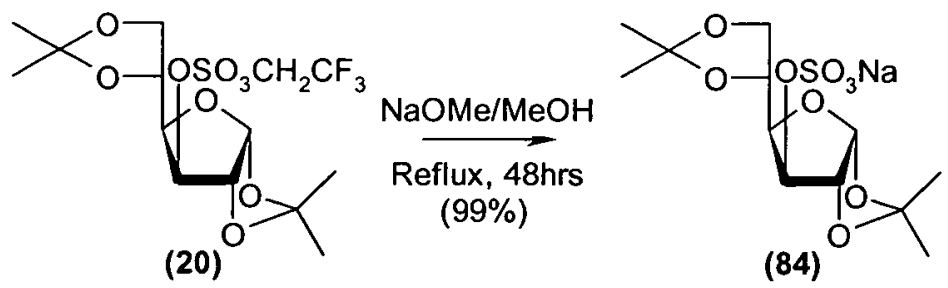

Figure 11:1: Sodium methoxide removal.

The trifluoroethyl sulfate ester was far more stable to methoxide than had been originally anticipated. From the outset it had been understood that there would be differences in reactivity between the sulfonyl esters and the sulfate diesters but this experiment highlights the variances in their reactivities.

When a related structure (30) was refluxed in a methanolic solution of sodium methoxide the outcome of the reaction was not as simple as above. A mixture of two products were observed. The desired sulfate (85) was isolated in only a $49 \%$ yield the other half of the starting material had reacted to form the 6-methyl ether (86) (see Figure 11:2).
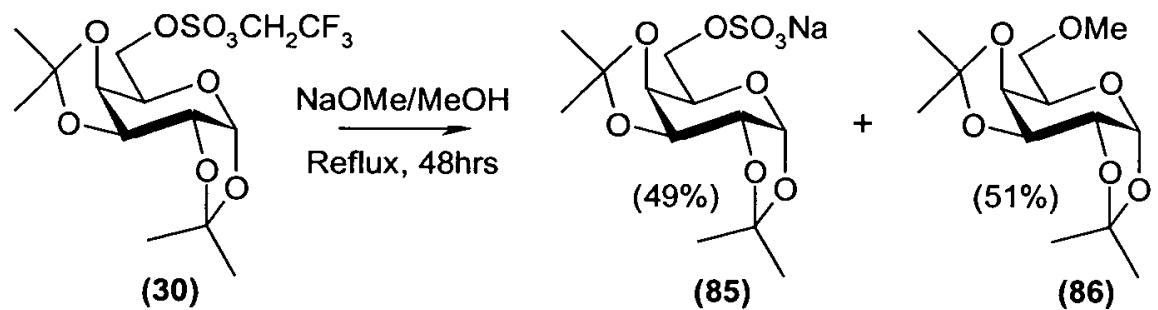

(86)

Figure 11:2: Deprotection of (30).

This is another example of the potential for the trifluoroethyl group to act as a leaving group. From this reaction it maybe possible to derive some conclusions about the mechanism for the deprotection with methoxide. It would appear that the reaction proceeds by an $\mathrm{S}_{\mathrm{N}} 2$ displacement mechanism at the carbon $\alpha$ to the sulfate. In the case of the sulfonyl esters there is only one site for such a displacement to take place however with the sulfate diesters there are two potential site of attack and the potential for the wrong carbon to be attacked and the incorrect product to be formed. When (20) was deprotected the sulfate is in a secondary position and consequently far less likely to undergo $S_{N} 2$ displacement than the primary sulfate of (30) (also the 
Section Two: Results and Discussion.

furanose from of (20) further reduces the conformational flexibility of the sugar ring permitting a very narrow angle of attack which inhibits the displacement further).

It would seem that removal of the sulfate protecting group by sodium methoxide is not suitably reliable.

\subsection{Other Bases for the Deprotection of Trifluoroethyl Esters.}

In the search for a reliable removal method a variety of other bases were considered. The results are shown in Table 11:7 below.

Table 11:7

\begin{tabular}{|c|c|c|c|c|}
\hline $\begin{array}{c}\text { Entry } \\
\text { No. }\end{array}$ & $\begin{array}{c}\text { Protected } \\
\text { Sulfate }\end{array}$ & Base & Conditions & Result \\
\hline 1 & $\mathbf{( 3 0 )}$ & tert $-\mathrm{BuOK}$ & tert $-\mathrm{BuOH}$, reflux $1 \mathrm{~h}$. & $\begin{array}{c}\text { Quantitative } \\
\text { yield }\end{array}$ \\
\hline 2 & $\mathbf{( 2 0 )}$ & $\mathrm{KHCO}_{3}$ & $\mathrm{MeCN}, 50^{\circ} \mathrm{C}, 16 \mathrm{~h}$ & No reaction \\
\hline 3 & $\mathbf{( 2 0 )}$ & $\mathrm{K}_{2} \mathrm{CO}_{3}$ & $\mathrm{MeCN}, \mathrm{TsOH}, 2 \mathrm{~h}$. & $\begin{array}{c}\text { Removes } \\
\text { isopropylidene } \\
\text { ring. }\end{array}$ \\
\hline 4 & $\mathbf{( 3 0 )}$ & $\mathrm{NaH}$ & $\mathrm{THF}, 16 \mathrm{~h}$. & No reaction \\
\hline 5 & $\mathbf{( 3 0 )}$ & $\mathrm{KOH}$ & $\mathrm{Bu}_{4} \mathrm{NBr}, \mathrm{DCM}, \mathrm{H}_{2} \mathrm{O}$, & No reaction \\
& & & $\mathrm{reflux}, 24 \mathrm{~h}$. & No reaction \\
\hline 6 & $\mathbf{( 3 0 )}$ & $\mathrm{NEt}_{3}$ & $\mathrm{BF}, 48 \mathrm{~h}$ & No reaction \\
\hline 7 & $\mathbf{( 2 0 )}$ & $\mathrm{Bu}_{3} \mathrm{SnOEt}_{3}$ & $\mathrm{THF}$, reflux, 48h & No reaction \\
\hline 8 & $\mathbf{( 3 0 )}$ & $\begin{array}{c}\text { Guanidine } \\
\mathrm{HONCHArNO}\end{array}$ & $\mathrm{THF}$, reflux, 1h & \\
\hline
\end{tabular}

One of the first reactions performed was to heat (30) with potassium tert-butoxide in tert-butanol (entry 1) this led to a quantitative yield of the sulfate in a surprisingly short period of time. This reaction will be more fully explored in the next section. 
There is no obvious reason for why potassium tert-butoxide should be so successful in this reaction. However the lack of reaction in the other experiments listed in Table 11:7 above can be used to draw some conclusions. Entries 2, 3 and 4 show that the reaction is not simply dependant on a strong non-nucleophilic base. Entry 5 also show that the success in entry 1 is due to potassium tert-butoxide and not to small amounts of potassium hydroxide formed from small amounts of water being present in the tert-butanol. Tributyltin alkoxides are often used when a highly nucleophilic base is required however entry 7 shows that increasing the nucleophilicity of the base has no effect on the protected sulfate.

As will be discussed in the next section the success of potassium butoxide maybe due in part to its potential for eliminating of a $\beta$-hydrogen. To test this theory the anion of 4-nitro benzaldoxime was used in an attempt to remove the trifluoroethyl group (entry 8 ). The guanidine salt of 4-nitro benzaldoxime is a highly nucleophilic base which will easily lose a $\beta$-hydrogen ${ }^{169}$ (see Figure 11:3) yielding a oxygen anion and 4-nitro benzonitrile. However as entry 8 shows the propensity to $\beta$-eliminate cannot be the key to the success of tert-butoxide as the 4-nitro benzaldoximate salt was unable to remove the trifluoroethyl protecting group.

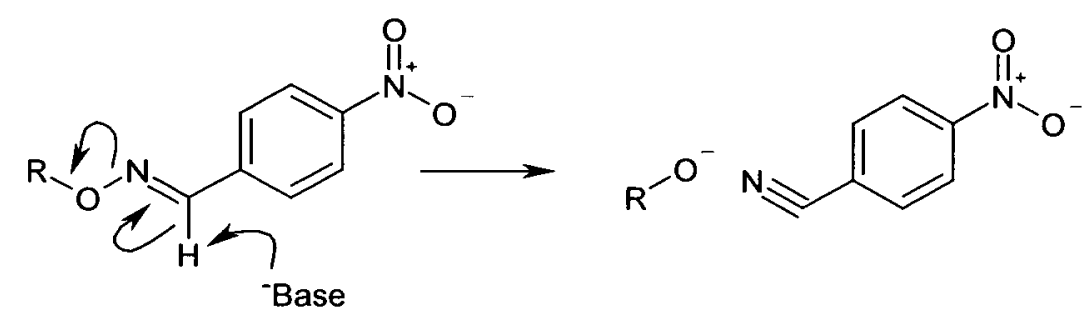

Figure 11:3: 4-Nitro benzaldoximate.

The only conclusion that can be made is that their is no simple reason why potassium tert-butoxide is so successful at removing the trifluoroethyl group. The mechanism for the deprotection reaction is not obvious and it is not influenced by the host of factors that have been varied. Potential mechanisms for this reaction have been examined and a fuller discussion of the them as well as a description of the experiments carried is included in the next section. 


\subsection{Potassium tert-Butoxide for the Deprotection of Trifluoroethyl Esters.}

A number of protected sulfates were treated with potassium tert-butoxide to determine how general the procedure was.

Both (20) and (30) were deprotected in excellent yield and without affecting the protecting groups (see Figure 11:4 and Figure 11:5).

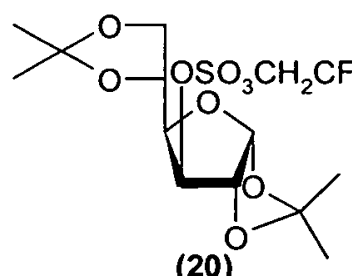

(20)

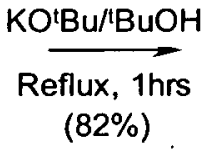

$(82 \%)$

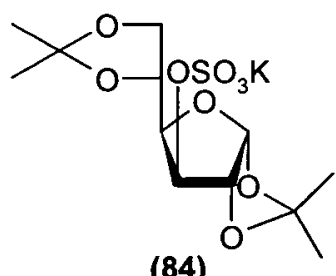

(84)

Figure 11:4: Sulfate deprotection of (20).
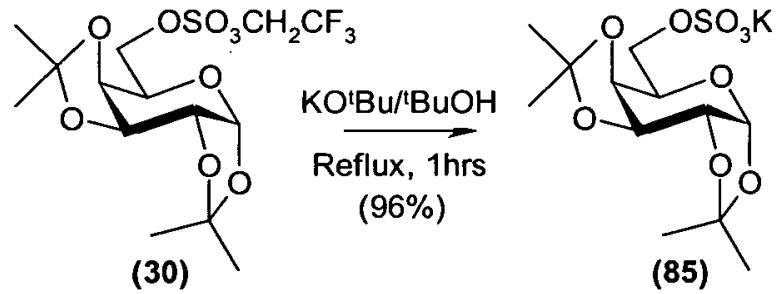

(85)

Figure 11:5: Sulfate deprotection of (30).

As expected the acetates of (29) were not stable to the deprotection conditions but a good yield $(88 \%)$ of the fully deprotected D-glucose 6-sulfate (87) was obtained (see Figure 11:6).

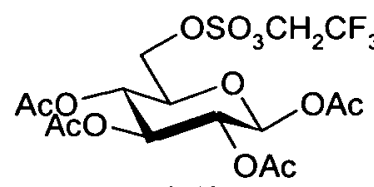

(29)

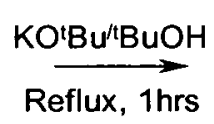

$(88 \%)$

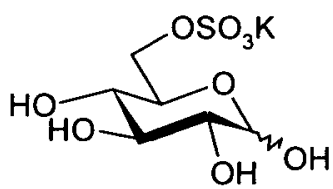

(87)

Figure 11:6: Sulfate deprotection of (29).

These three reactions have been published ${ }^{134}$ see Appendix 1. 
When (43) was treated with potassium tert-butoxide a new product (88) that was less polar than the starting material was observed as well as the expected sulfate salt (89). After aqueous work up of the reaction mixture and column chromatography only the sulfate was isolated. It is possible that the initially product formed is in fact the cyclic sulfate $^{70}$ (see Figure 11:7).

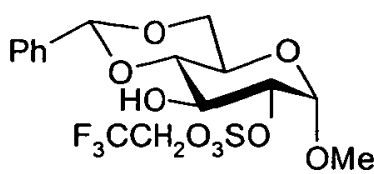

(43)

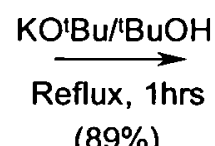

$(89 \%)$

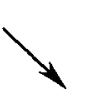

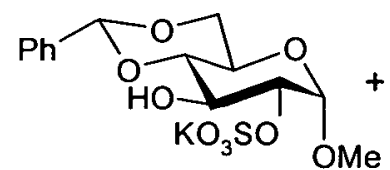

(89)

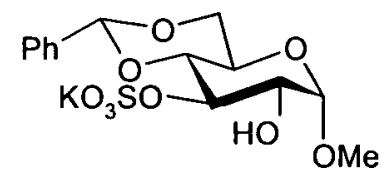

(90)

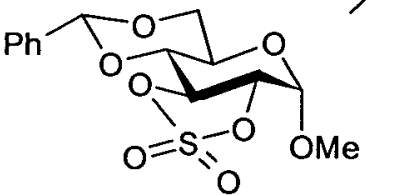

(88)

Figure 11:7: Sulfate deprotection of (43).

If the deprotection reaction precedes via the cyclic sulfate there must be a doubt over the final position of the sulfate. Migrations of this type are well known to occur with other esters such as acetates under basic conditions.

To identify if the sulfate did migrate, a simple experiment was carried out. (43) was treated with potassium tert-butoxide and worked up in the normal way to give the sulfate. The resulting sample was then treated once again with trifluorodiazoethane. The mixture was examined by $\mathrm{nmr}$ for the presence of different protected sulfate diesters. Only the 2-trifluoroethyl sulfate (43) could be identified and there was no trace of the 3-sulfated compound. It would appear that the cyclic sulfate is a minor side product of the reaction and has no real effect on the outcome of the reaction. However the reaction may also be explained by the cyclic sulfate being hydrolysed preferentially to the thermodynamically more stable 2-sulfate. The experiment described above cannot differentiate between these two explanations but it seems unlikely that the hydrolysis of the cyclic sulfate would be entirely dominated by one product without a small amount of the other isomer being formed. 
Based on these results, heating with potassium tert-butoxide in butanol would be the method of choice for the removal of the trifluoroethyl group. The reaction is highly successful although the potential for sulfate migration must considered.

\subsection{Other Attempts at Deprotection Conditions.}

This section details a number of failed attempts at alternative deprotection strategies. Further examples that led to unexpected results can be found in a latter section.

As was described in the preliminary work, the ideal method to remove the trifluoroethyl group would be to employ the condition used by Woodward ${ }^{105}$ to remove the trichloroethyl group. As described previously the trifluoroethyl sulfates are stable to these conditions (see Figure 11:8).

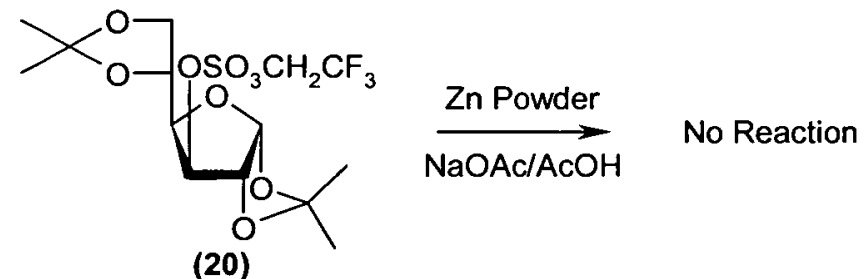

Figure 11:8: Woodward's conditions.

The exact mechanism for this reaction is not clear but it has been speculated that the reaction proceeds via a single electron transfer process resulting in radical intermediates. With this in mind compound (29) was refluxed with tributyltin hydride and azobisisobutyrylnitrile (AIBN) a mixture known to be a reductive source of free radicals. No reaction was observed even after the reaction had been continued for a prolonged period of time (see Figure 11:9).

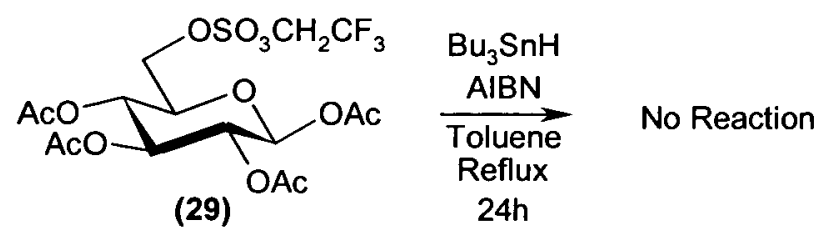

Figure 11:9: Tributyl tin reaction. 
When the trifluoroethyl group is removed from a sulfate using sodium methoxide as the base the mechanism is believed to be a $S_{N} 2$ displacement. In an attempt to exploit this mechanism other nucleophiles were tested. As detailed earlier the trifluoroethyl protected sulfates were not affected by exposure to tertbutylammonium fluoride (TBAF), bromide (TBAB) or iodide (TBAI) which are all known sources of halogen nucleophiles. Another powerful method for generating nucleophilic iodide ions is to mix a solution of sodium iodide and 18-Crown-6, the crown ether complexes to the sodium ions and effectively removes them from the solution so freeing the iodide ion and enhancing its nucleophilicity. Compound (29) was added to a solution of sodium iodide and 18-crown-6 in acetone however no reaction was observed (see Figure 11:10).

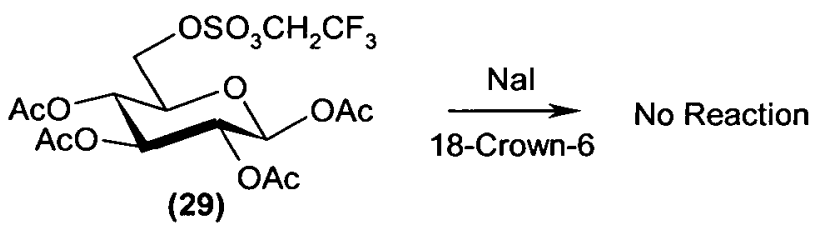

Figure 11:10: Sodium iodide and crown ether.

None of these reactions gave any indication of the desired product being formed.

\subsection{Oxidative Cleavage.}

The published chemistry of the trifluoroethyl groups is very limited indeed. The only feature of their chemistry is their ability to undergo a dehydrofluorination reaction under strongly basic conditions ${ }^{170}$. The overall effect is the loss of hydrogen fluoride to yield a difluoro alkene (see Figure 11:11).

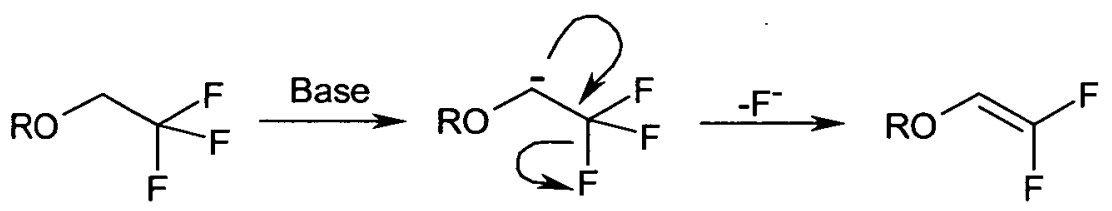

Figure 11:11: Dehydrofluorination.

When the dehydrofluorination reaction was attempted on (30) using a single equivalent of butyllithium the desired product (91) was isolated as a 1:1 mixture with 
the starting material which had an identical chromatographic properties. When the reaction was rerun using 2 equivalents of butyllithium, an (80\%) yield of pure (91) was obtained (see Figure 11:12).

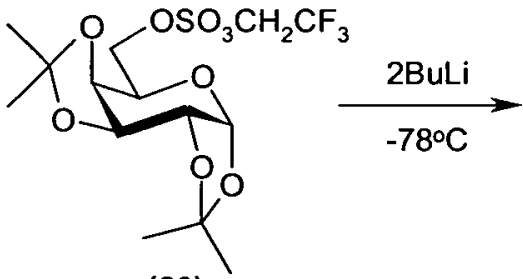

(30)

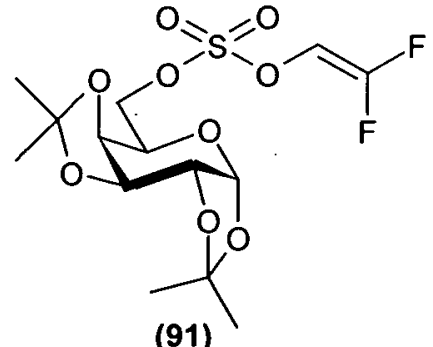

(91)

Figure 11:12: Dehydrofluorination of (30).

Initially the necessity for two equivalents of base suggested a more complex mechanism than that outlined in Figure 11:11 however the reason a second equivalent is required is actually quite trivial. The proton on the difluoroalkene formed in the reaction is more acidic than the protons in the trifluoroethyl group so in basic solution the difluoroalkene exists as a carbanion. The formation of this difluoroalkene carbanion consumes a mole of base, consequently 2 equivalents of the base are required to drive the reaction to completion. Allcock, Suszko and Evans ${ }^{171}$ have published an elegant demonstration of this. The paper contains a series of ${ }^{19} \mathrm{~F}$ nmr spectra of a trifluoroethyl containing compound. The series follows the addition of butyllithium (up to 2 equivalents) and then the partial quenching of the reaction. This beautifully shows the change from the singlet associated with the trifluoroethyl group to a pair of doublets corresponding to the difluoro alkene carbanion as base is added. As the reaction is quenched the pair of doublets from the difluoroalkene carbanion are replaced by a pair of doublets that are associated with the final difluoroalkene.

In an attempt to discover an alternative deprotection route (91) was added to a solution of potassium permanganate in acetone. The difluoroalkene was successfully cleaved to give the sulfate (see Figure 11:13) as observed by TLC (single spot to single spot). 


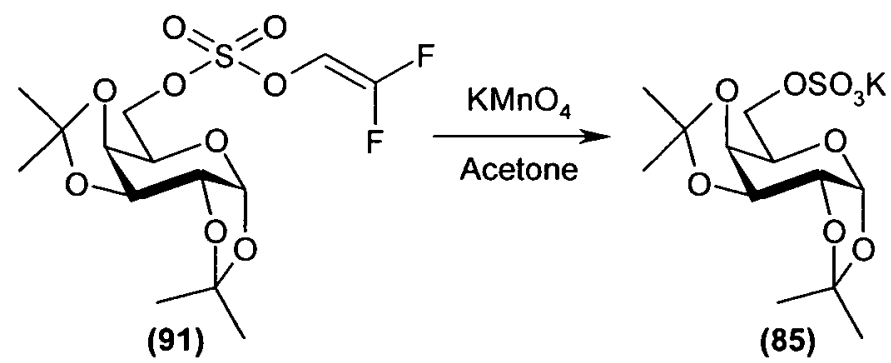

Figure 11:13: Permanganate cleavage.

This reaction is awkward from a practical point of view as the sulfates formed require extensive purification to remove the excess potassium permanganate and sludge like manganese dioxide.

As an alternative method for the oxidative cleavage (91) was treated with ozone at low temperature. After the reaction with ozone thiourea was added to destroy the ozonide and hypoperoxididic products formed. Thiourea was chosen because the resulting insoluble thiourea S-dioxide could be filtered from the reaction mixture ${ }^{172}$. Subsequent addition of potassium carbonate to the reaction solution permitted the sulfate to be isolated as its potassium salt in good yield (77\%).

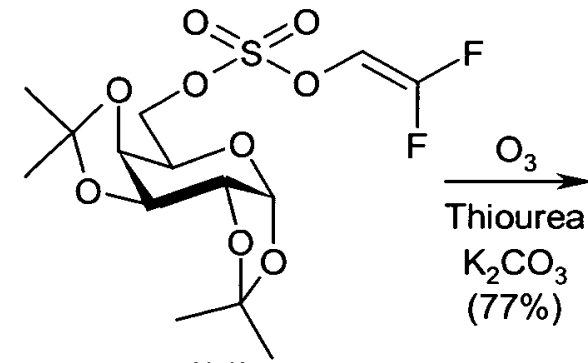

(91)

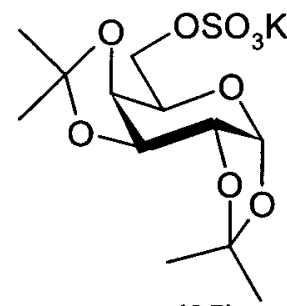

(85)

Figure 11:14: Ozone cleavage.

It was possible to combine the dehydrofluorination step and the oxidative cleavage into a single procedure. Firstly (30) in THF was treated butyllithium at $-78^{\circ} \mathrm{C}$. After $15 \mathrm{~min}$ the reaction was quenched with methanol. Lithium salts were precipitated by the addition of diethyl ether. The solvent was then removed and replaced with methanol. The mixture was treated with ozone at $-12^{\circ} \mathrm{C}$ for $1 \mathrm{~h}$. The mixture was flushed with oxygen and then warmed to $0^{\circ} \mathrm{C}$ thiourea and potassium bicarbonate 
were then added. The solvent was then removed and the product purified by column chromatography (see Figure 11:15).

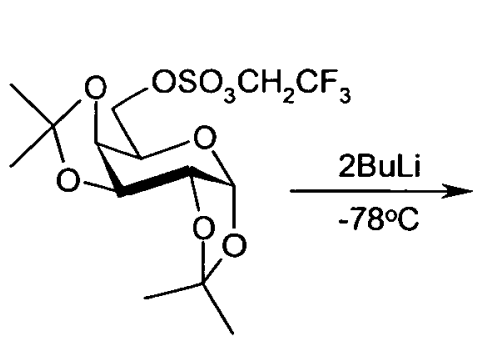

(30)

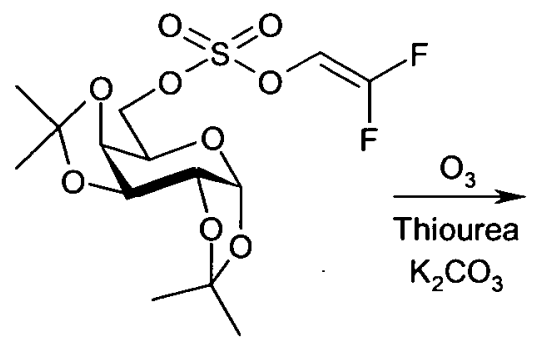

(91)

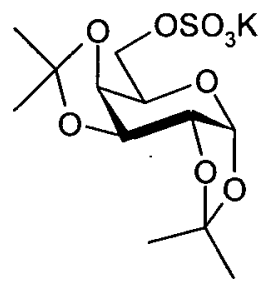

(85)

$(89 \%)$

Figure 11:15: Oxidative deprotection strategy.

His reaction sequence led to the desired sulfate in good (89\%) yield over the two steps. This is a highly successful strategy for deprotecting the protected sulfates. It is more complex than the potassium tert-butoxide protocol but it offers a viable alternative.

\subsection{Conclusions.}

This section has demonstrated that the trifluoroethyl group can be reliably removed from a protected sulfate in excellent yields. Both procedures developed produce the sulfate salts in a from that simplifies purification and does not require ion exchange chromatography.

Although treating the protected sulfates with potassium tert-butoxide in hot tertbutanol could not be described as a mild procedure the conditions are not overly harsh. Most of the monosaccharides examined survived unchanged and the conditions should not affect glycosidic linkages. Indeed potassium tert-butoxide in DMSO is commonly used in carbohydrate chemistry to isomerise allyl groups to enol ethers prior to their removal ${ }^{173}$. 
The potassium tert-butoxide route is the protocol of choice. It is highly reliable and high yielding although the possibility of sulfate migration maybe a potential problem that should be considered. The oxidative cleavage protocol is more involved but still provides a viable alternative to the primary route. As the dehydrofluorination step is conducted at low temperature there is a reduced chance of sulfate migration.

\section{Mechanistic Studies.}

The previous section demonstrated that heating a trifluoroethyl sulfate diester in a solution of potassium tert-butoxide would remove the trifluoroethyl group. The mechanism for this reaction is not obvious. In the preceding section an attempt was made to identify which properties of potassium tert-butoxide, such as strength and nucleophilicity influenced the reaction as such information could help identify possible mechanisms. No single property was found that would influence the outcome of the reaction. The reaction mechanism must therefore be complex and potentially involves a combination of the base's properties.

It should be noted that the choice of potassium tert-butoxide as a base was highly fortuitous. If the mechanism for the butoxide initiated deprotection can be elucidated then the knowledge gained could be used to discover milder reaction conditions.

This section describes the experiments carried out to identify the mechanism of the deprotection step. It will begin by discussing some simple potential mechanisms involving direct nucleophilic displacement and then continue into more complex mechanisms involving multiple steps.

\subsection{Simple Nucleophilic Displacements.}

These two sections consider possible mechanisms involving direct displacement of the trifluoroethyl group. There are two possible sites of attack that would lead to the sulfate being displaced. Both attack at carbon $\alpha$ to the sulfate [route (b)] in Figure 
12:1 and at the sulfur of the sulfate [route (a)] in Figure 12:1 would lead eventually to the formation of a sulfate monoester.

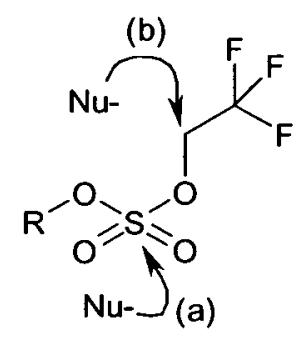

\section{Figure 12:1: Alternative $S_{\mathrm{N}} 2$ displacements.}

However the two mechanisms would lead to the formation of different by-products. These compounds should be identifiable. The presence of particular by-products would be strong evidence for a particular mechanism.

\subsubsection{Nucleophilic attack upon carbon.}

The most obvious mechanism for the removal of the trifluoroethyl group by an $\mathrm{S}_{\mathrm{N}} 2$ displacement of the sulfate by the butoxide nucleophile (see Figure 12:2). If such a mechanism is taking place then it should be possible to detect quantities of $2^{\prime}, 2^{\prime}, 2^{\prime}-$ trifluoroethyl tert-butyl ether (92) in the crude reaction mixture.

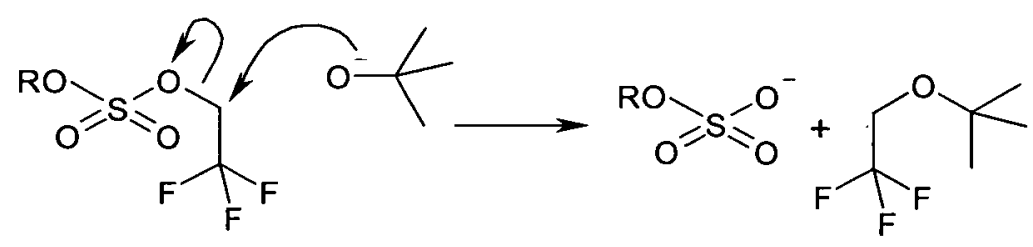

(92)

Figure 12:2: $\mathrm{S}_{\mathrm{N}} 2$ displacement on carbon.

The reaction of (30) with potassium tert-butoxide was monitored for the formation of 2',2',2'-trifluoroethyl tert-butyl ether (92). No trace of the ether was observed by TLC or MS (electrospray) of the crude reaction mixture.

As the butyl ether (92) may be volatile and could evaporate from the reaction mixture, the volatile components of the reaction were also collected. A stream of nitrogen gas was passed through the condenser of the reaction and the effluent gas was passed through a vessel cooled to $-78^{\circ} \mathrm{C}$ by a $\mathrm{CO}_{2}$ (solid) $/$ acetone bath. Some 
condensate was collected and was identified as iso-butene (94) by addition to a bromine solution in dichloromethane. The dibromo-derivative (93) formed was characterised by ${ }^{1} \mathrm{H} \mathrm{nmr}$. The iso-butene is assumed to have arisen from tert-butanol by elimination of a $\beta$-hydrogen and an oxygen leaving group (see Figure 12:3).

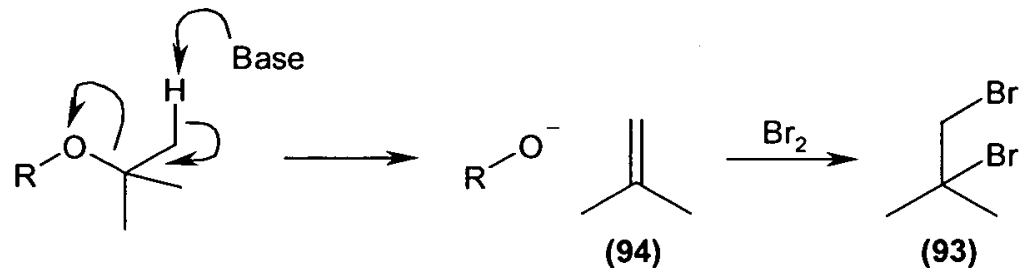

Figure 12:3: $\beta$-Hydrogen elimination.

The deprotection reaction of (30) was then reinvestigated for the presence of 2,2,2trifluoroethanol (10) but none was found.

No evidence has been found that would support a mechanism involving nucleophilic displacement of a sulfate by a butoxide nucleophile attacking at carbon. This mechanism was doubtful from the outset as, as was shown in the previous section, increasing the nucleophilicity of the base has no effect on the outcome of the reaction.

The nucleophilicity of the base would be unimportant if the mechanism involved an $\mathrm{S}_{\mathrm{N}} 1$ process however the lack of appropriate by-products also rules this mechanism out.

Tert-butoxide is a poor nucleophile for electrophilic carbon centres and would not normally be expected to behave in a nucleophilic fashion. In addition to this the carbon $\alpha$ to the sulfate is not highly electrophilic due to the electronic effects of the neighbouring fluorine atoms. After all this stability to nucleophiles is the basis on which the sulfate diester protecting group was designed. 


\subsubsection{Nucleophilic attack upon sulfur.}

Another obvious potential mechanism would be for the nucleophile to attack the sulfur atom of the protected sulfate, displacing trifluoroethanol. The resulting tertbutyl sulfate diester could be expected to collapse as shown in Figure 12:3. This would account for the formation of the iso-butene during the reaction. However the evidence for this mechanism is compromised by the lack of the expected trifluoroethanol by-product in the reaction mixture.

There is some literature precedence for butoxide ions attacking high oxidation state sulfur centres. Chang 174 noted the retention of stereochemistry during an alkaline hydrolysis of $3 \beta$-cholestanol mesylate (94) and attributed this unexpected result to the attack of butoxide ions on the sulfur of the mesylate group (see Figure 12:4).<smiles>CC(C)CCCC(C)C1CCC2C3CCC4C[C@@H](O)CCC4(C)C3CCC12CC(C)(O[Na])OC(C)(C)C</smiles>

Figure 12:4: Hydrolysis of $3 \beta$-cholestanol mesylate.

If the butoxide ion is attacking the sulfur atom as shown in Figure 12:5 then it is obvious that one of the oxygen atoms in the sulfate product originated in the nucleophile. This mechanism could by validated by introducing an uncommon isotope of oxygen into the butoxide base. Mass spectrometry could then be used to detect incorporation of the isotope into the product sulfate.

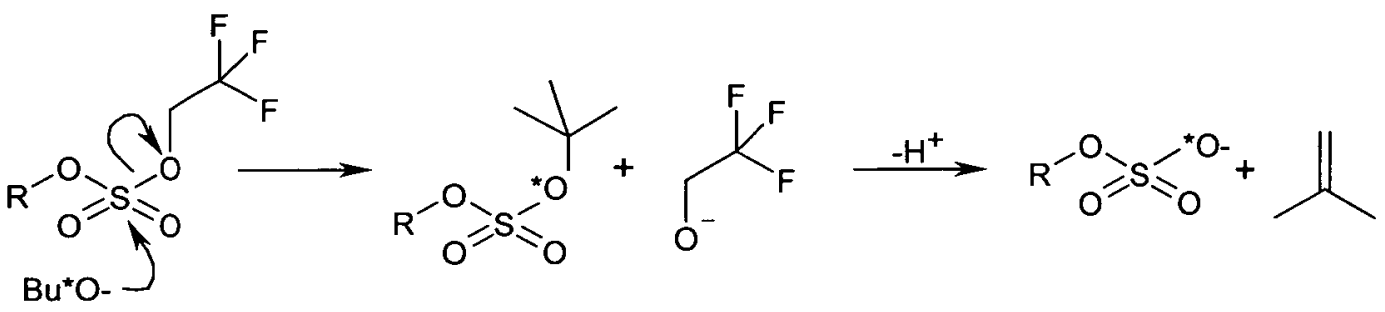

Figure 12:5: Nucleophilic attack on sulfur. 
In an attempt to corroborate this theory the deprotection of (30) was performed using $10 \%{ }^{18} \mathrm{O}$ labelled tert-butanol as both the source of the butoxide ions and the solvent. The labelled solvent was produced by hydrolysis of tert-butyl chloride in $10 \% \mathrm{H}_{2}{ }^{18} \mathrm{O}$. The deprotection of (30) was examined by electrospray MS and the ratio of the isotope peaks for the labelled and non labelled experiments were compared. No incorporation of ${ }^{18} \mathrm{O}$ into the sulfate (85) was observed.

This result effectively rules out a mechanism where the sulfur of the sulfate is attacked by butoxide ions or indeed any solvent derived oxygen atom.

\subsection{Mechanisms Involving Multiple Steps.}

It has been commented previously that the only chemistry of note concerning trifluoroethyl groups is their ability to undergo dehydrofluorination reactions. As shown this reaction usually requires a strong lithium derived base however potassium butoxide should be strong enough to perform the same function (see Figure 12:6). If so then it is possible that the deprotection reaction involves a multiple step process in which the loss of hydrogen fluoride is the initial step.

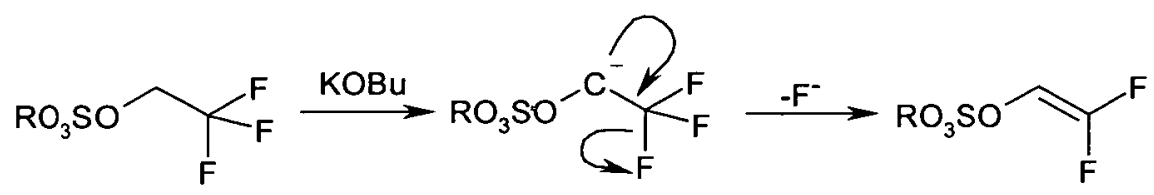

Figure 12:6: Dehydrofluorination of sulfates.

An experiment was designed to identify the difluoro alkene as an intermediate in the reaction. (30) was deprotected with potassium $\mathrm{d}^{10}$ tert-butoxide in deuterated tertbutanol and the reaction was monitored by nmr.

The reaction was performed in a sealed $\mathrm{nmr}$ tube. The mixture of protected sulfate and base was examined by ${ }^{1} \mathrm{H} \mathrm{nmr}$ before the vessel had been heated. This showed that the two protons on the methylene of the trifluoro ethyl group had been 
exchanged with the solvent and could not be followed through the reaction. However the experiment could be monitored by ${ }^{13} \mathrm{C} \mathrm{nmr}$.

The sealed tube was heated at $80^{\circ} \mathrm{C}$ for $30 \mathrm{~min}$. The ${ }^{13} \mathrm{C} \mathrm{nmr}$ was then re-examined, a new peak was visible at $160 \mathrm{ppm}$ but was a singlet not the doublet of doublet expected for the difluoroalkene. The ${ }^{19} \mathrm{~F} \mathrm{nmr}$ of the reacted mixture showed no ${ }^{19} \mathrm{~F}$ peaks at all! When the tube was opened the contents were found to contain one new product that was not the sulfate sodium salt nor the difluoroalkene. The compound was identified by $\mathrm{nmr}$ and from its $\mathrm{X}$-ray crystal to be the 5,6-dehydrated derivative of the parent sugar (96) ${ }^{175}$ (see Figure 12:7). See Figure 12:8 and Appendix 2 for crystal structure and data.
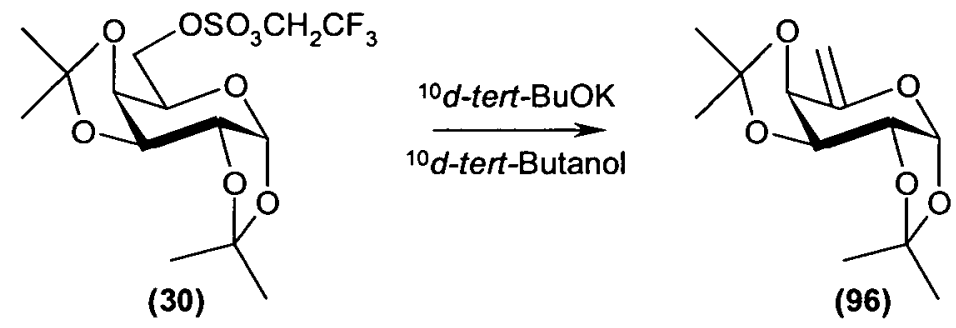

Figure 12:7: Dehydration of (30).

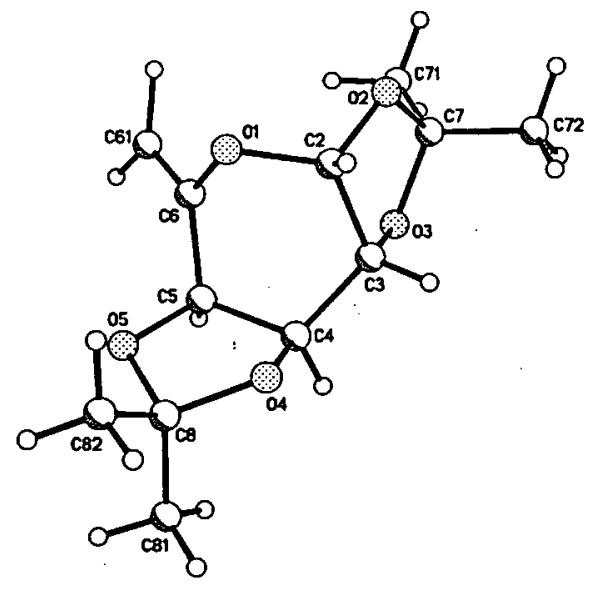

Figure 12:8: X-ray crystal structure of (96).

This product is obviously derived from an alternative elimination reaction (see Figure 12:9) where the hydrogen at the 5-position of the sugar is abstracted by the 
base and a sulfate monoester is eliminated to form a double bond. Why the reaction should proceed in this direction when deuterated potassium tert-butoxide is used and not to the desired sulfate is curious. The diversion of the mechanisms must be due either, to a kinetic isotope effect in the rate limiting step or the fact the deuterated tert-butoxide is probably slightly more basic than the undeuterated alkoxide.
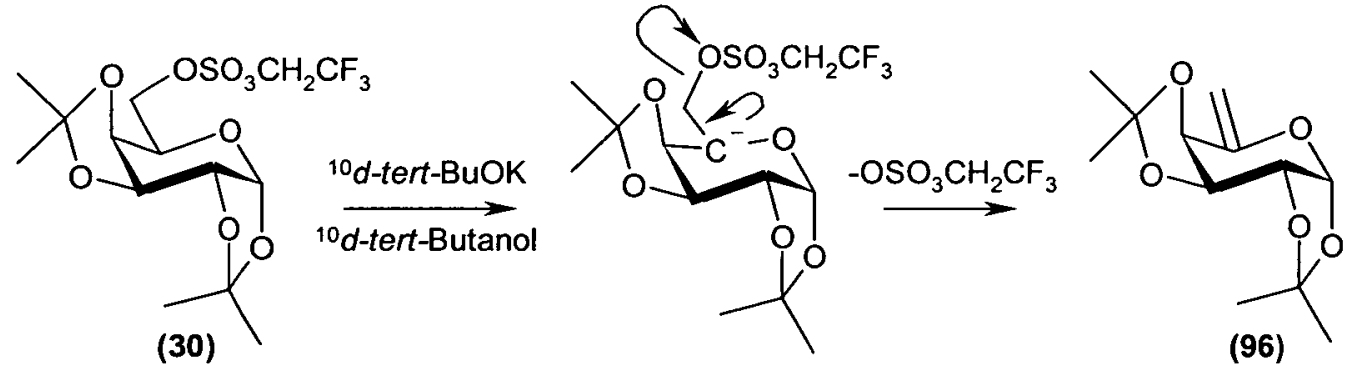

Figure 12:9: Alternative elimination reaction.

This experiment obtained no information that could be used in determining whether or not the difluoroalkene was an intermediate in the reaction. It is highly interesting that no fluorinated organic compounds could be detected in the reaction mixture and this point will be explored later in the section.

Although there is no evidence to corroborate the intermediary of a difluoroalkene in the deprotection reaction there is none to refute it either. The difluoroalkene (91) has be found to give the sulfate when reacted with tert-butoxide, even at room temperature (see Figure 12:10).

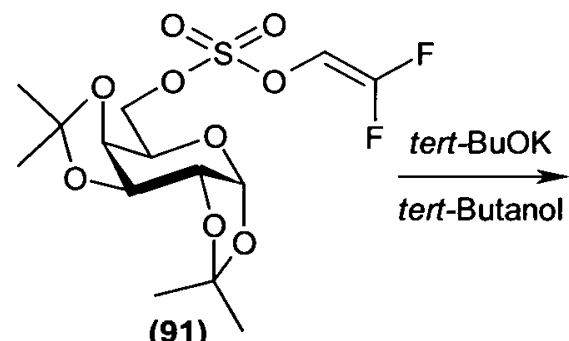

(91)

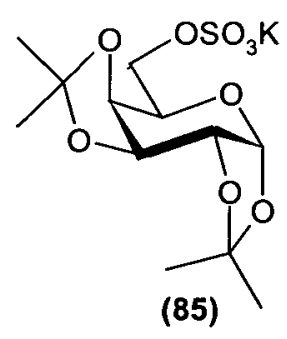

(85)

Figure 12:10: Difluoroalkene removal.

Given the literature precedence for the difluoroalkene being formed and the reactivity of the compound it would seem likely that the initial stage of the deprotection reaction is the dehydrofluorination. There are a number of possible ways by which 
the difluoroalkene formed could collapse to the sulfate some of which are examined in the next sections.

\subsubsection{Carbenes.}

As was discussed in the previous section, the difluoroalkene intermediate will exist as a carbanion in basic solution. If this carbanion was to spontaneously fragment then the overall effect is that of $\alpha$-elimination and the by-product formed is a highly reactive carbene (see Figure 12:11).
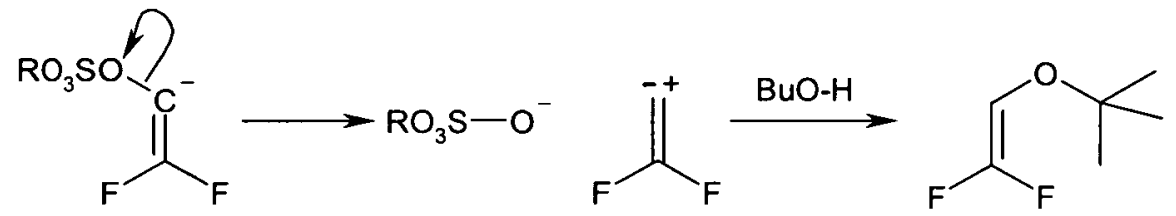

(97)

Figure 12:11: $\alpha$-Elimination to give carbene.

The carbene formed would then react with the solvent, inserting into the oxygen hydrogen bond to give the enol ether (97) (see Figure 12:11). It should be noted that the rearrangement of the difluorovinylidene where a fluorine migrates to form the difluoroacetylene has been ruled out by theoretical calculations 176 .

Although no trace of the enol ether could be detected in the deprotection reaction a number of experiments were carried out to trap any carbene formed in the reaction. The hypothesised carbene is known as difluorovinylidene. This compound has been extensively examined, its theoretical existence was postulated in the early 1970 's 177 and it had been isolated in inert low temperature matrices by the mid 1970's 178 . The species' behaviour is in keeping with other similar compounds ${ }^{179}$ and a recent article has reviewed its syntheses and its spectroscopic properties. However no one has yet published a practical reaction that involves difluorovinylidene inserting into an organic compound. With no comparisons in the literature three common carbene traps were chosen and were incorporated into the trifluoroethyl deprotection reaction. The results are shown in Table 12:8. 
Table 12:8: Carbene traps.

\begin{tabular}{|c|c|c|c|}
\hline Protected Sulfate & Carbene Trap & Conditions & Result \\
\hline $\mathbf{( 3 0 )}$ & Cyclohexene & tert-BuOK, Reflux & No insertion \\
\hline $\mathbf{( 3 0 )}$ & Styrene & tert-BuOK, Reflux & No insertion \\
\hline $\mathbf{( 3 0 )}$ & Triethyl-silane & tert-BuOK, Reflux & No insertion \\
\hline
\end{tabular}

No insertion products or rearranged insertion products could be identified from the reactions in Table 12:8.

Although the difluorovinylidene compound may be too electron deficient to insert into these traps the evidence suggests that no carbene or carbenoid intermediates are formed during the deprotection reaction.

12.2.2. Nucleophilic displacement.

An obvious mechanism that must be considered is nucleophilic attack of the butoxide ion onto the difluoroalkene. The regioselectivity of such an attack is determined by the relative stability's of the two carbanion formed ${ }^{180}$ see Figure 12:12.

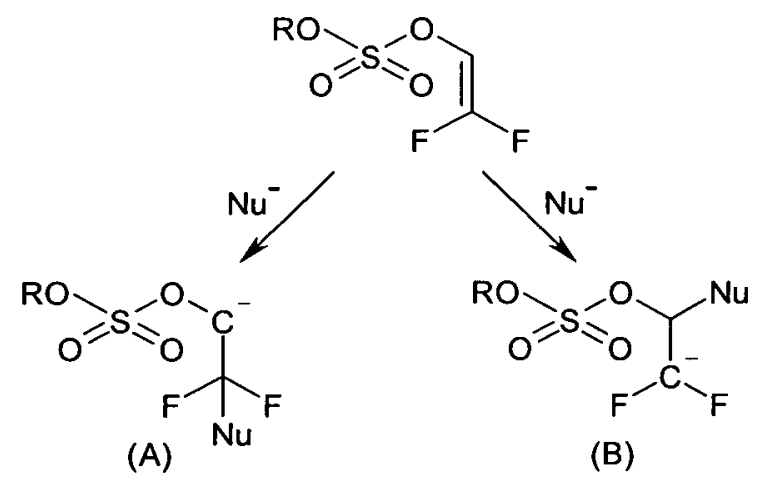

Figure 12:12: Regioselectivity.

In this case it is difficult to predict in which position the negative charge is going to receive the most stabilisation. There appears to be little difference in the energies of the two forms. Which ever of the two carbanions is formed has two possible fates. It could (a) abstract a proton from the solvent to give a saturated ether or it could (b) 
eliminate one of its groups (in this case fluorine or sulfate monoester) to give an enol ether. Overall, process (a) is an addition reaction and process (b) is an additionelimination reaction. Although there appears to be little difference between the two carbanions (A) and (B) the only obvious way for the deprotection reaction to occur is for the nucleophile to attack at the carbon $\alpha$ to the sulfate giving carbanion (B) and for the carbanion to under go an elimination to give the sulfate monoester and the 2,2-difluoroethyl tert-butyl enol ether (97).

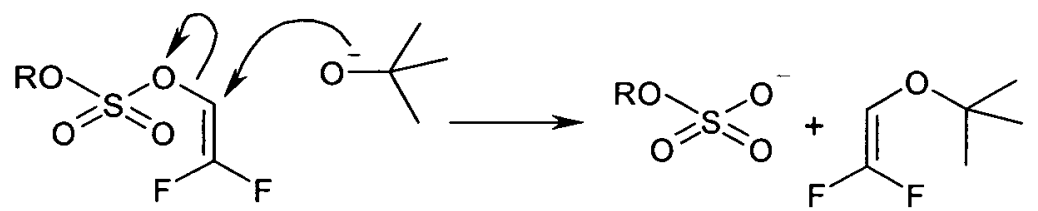

(97)

Figure 12:13: $S_{\mathrm{N}} 2$ displacement on difluoroalkene.

No trace of the tert-butyl ether (97) was found in the reaction mixture nor any difluoroacetaldehyde which is the expected product of the base catalysed fragmentation of the ether (97) (see Figure 12:14).

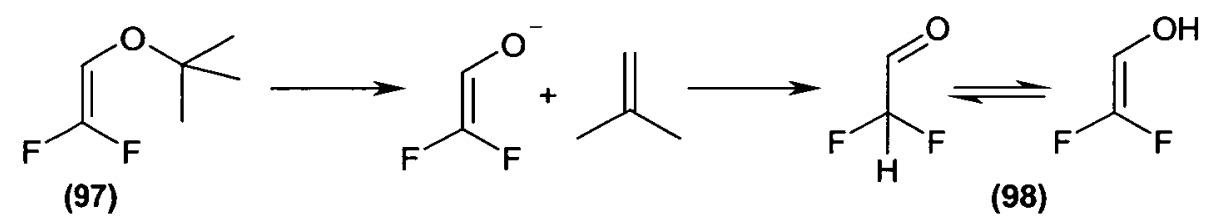

Figure 12:14: Difluoroacetaldehyde.

Difluoroacetaldehyde (98) is an extremely volatile compound ${ }^{181}$ (bp $27-28^{\circ} \mathrm{C}$ ) and consequently would not be expected to have been observed by mass spectroscopy. However the compound can be derivatised to form its 2,4-dinitro-phenylhydrazone by adding it to Brady's reagent solution (2,4-dinitro-phenyl hydrazine hydrochloride solution) ${ }^{182}$. When the volatile components of the deprotection reaction of (30) were passed through a chilled solution of 2,4-dinitrophenyl hydrazine hydrochloride no precipitate was obtained.

There is no experimental evidence in support of this mechanism. Although there appears to be little between the energy levels of the two carbanion there is 
considerable literature precedence against the nucleophile attacking the carbon $\alpha$ to the sulfate. There are numerous examples in the literature of nucleophiles attacking at the difluoromethylene group implying that carbanion (A) is the more stable of the two forms ${ }^{183}$.

In order to differentiate which of the two carbanions is the more stable an experiment was performed. The difluoro alkene (91) was reacted with a variety of nucleophiles at room temperature. The results of the reaction were observed by mass spectrometry by injecting crude samples of the reaction mixture directly in to an electrospray mass spectrometer. The results are shown in Table 12:9.

Table 12:9: Reaction of (91) and bases.

\begin{tabular}{|c|c|c|c|}
\hline Difluoroalkene & Base & Conditions & Result \\
\hline $\mathbf{( 9 1 )}$ & $\mathrm{NaOMe}$ & $\mathrm{MeOH}, \mathrm{RT}$ & Addition. \\
\hline $\mathbf{( 9 1 )}$ & $\mathrm{NaOEt}$ & EtOH, RT & $\begin{array}{c}\text { Mixture of addition } \\
\text { and elimination. }\end{array}$ \\
\hline $\mathbf{( 9 1 )}$ & tert-BuOK & tert-BuOH, RT & Elimination. \\
\hline
\end{tabular}

The mass spectrometer could easily identify the addition and elimination products of the parent difluoroalkene associated with each base. By rerunning the samples with the mass spectrometer in -ve ion mode it was possible to test for the presence of the sugar sulfate (i.e. (85)). The sulfate was detected in the butoxide and ethoxide samples but not in the methoxide it could therefore be inferred that the formation of an addition-elimination product is an important stage in the deprotection mechanism. The fact that addition-elimination products of the parent difluoroalkene are found but not the difluoroethene tert-butyl enol ether (97) must mean that the nucleophile attacks the difluoromethylene group (see Figure 12:15). 

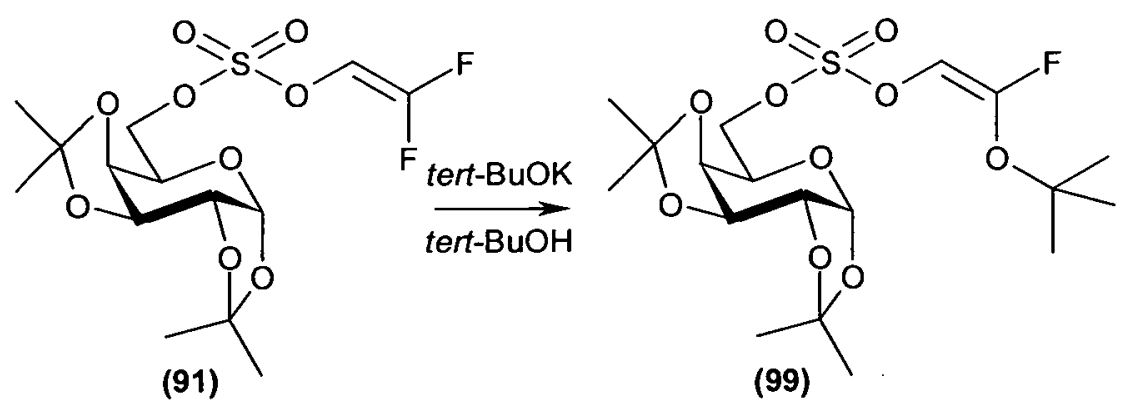

Figure 12:15: Butoxide addition/elimination product.

To obtain further proof for the structure of these compounds the reaction of potassium butoxide with (91) was quenched at a stage when both the addition (100) and the addition/elimination (99) products were present (see Figure 12:16). $\mathrm{A}^{19} \mathrm{~F} \mathrm{nmr}$ spectra of the crude products was obtained.
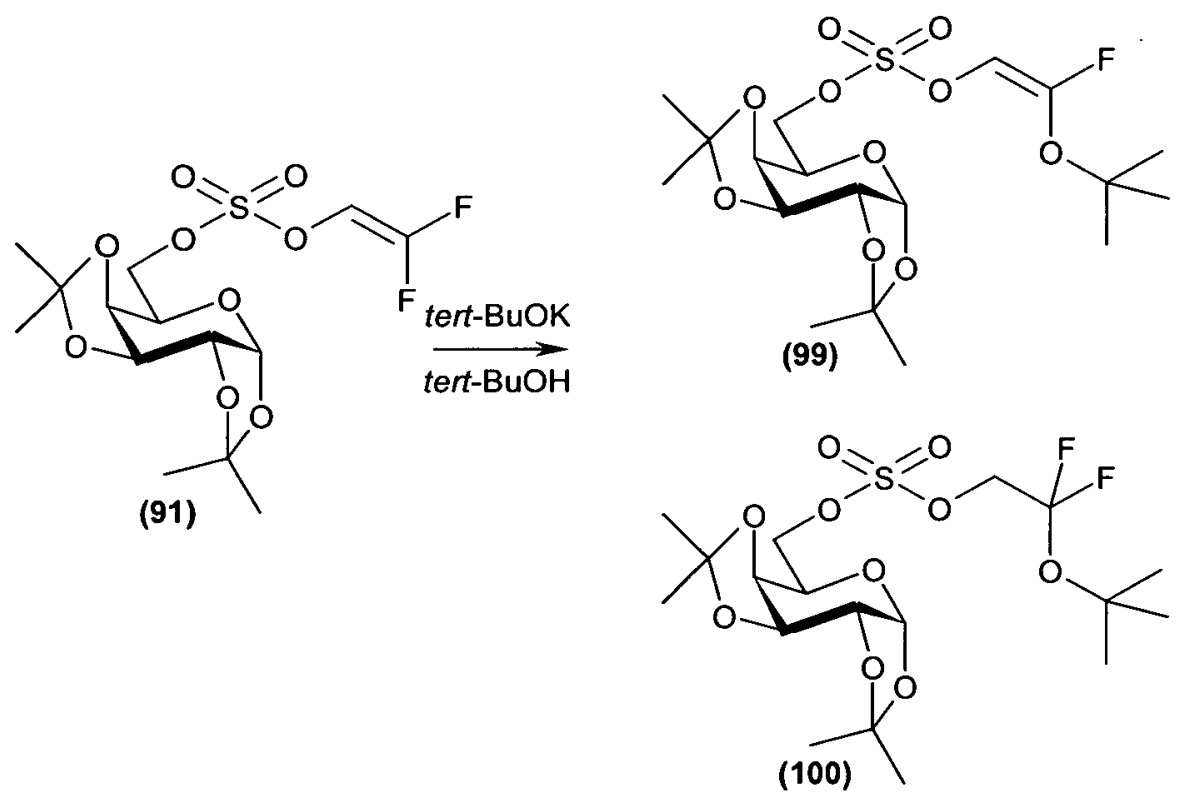

Figure 12:16: Addition and addition/elimination products.

The spectra clearly shows an "ab" system (centred at $-73.18 \mathrm{ppm}$ ) associated with the addition product (100) and a singlet $(-99.68 \mathrm{ppm})$ from the addition-elimination product (99). When the spectra was rerun without the proton decoupling each peak of the ab system was further split into a triplet and the singlet was split into a doublet. This is consistent with the proposed structures of (99) and (100). 
Furthermore when the singlet derived from the single fluorine atom in (99) was allowed to couple to the proton a coupling constant of $3.5 \mathrm{~Hz}$ was observed this strongly suggests that the fluorine and the hydrogen are in a cis-relationship to one another (if there was a trans-relationship the coupling constant should be around 20Hz). This indicates that (99) has the structure shown in Figure 12:16. Depuy and Schultz found very similar results when they reacted 1,1,1-trifluoro-2-phenyl ethane with potassium tert-butoxide (see Figure 12:17).<smiles>FC(F)(F)Cc1ccccc1</smiles><smiles>FC(F)=Cc1ccccc1</smiles><smiles>COc1ccccc1/C=C(/F)c1ccccc1</smiles>

Figure 12:17: Reaction of 1,1,1-trifluoro-2-phenyl ethane.

There is good evidence that (98) is an intermediary structure in the deprotection reaction of (30) with potassium tert-butoxide but there is no obvious way for (98) to collapse to give the sulfate monoester.

\subsubsection{Further dehydrofluorination.}

If the monofluoro-enol ether (98) was to undergo a further dehydrofluorination reaction the product would be an unsymmetrical acetylene diether. Such acetylene ethers are known to undergo nucleophilic displacements even with very poor nucleophiles ${ }^{184}$ such as the phenyl and butyl anions (from the appropriate lithium base). Ishikawa and co-workers have reported the synthesis of symmetrical and unsymmetrical acetylenes from trifluoroethyl ethers ${ }^{185}$ see Figure 12:18. 


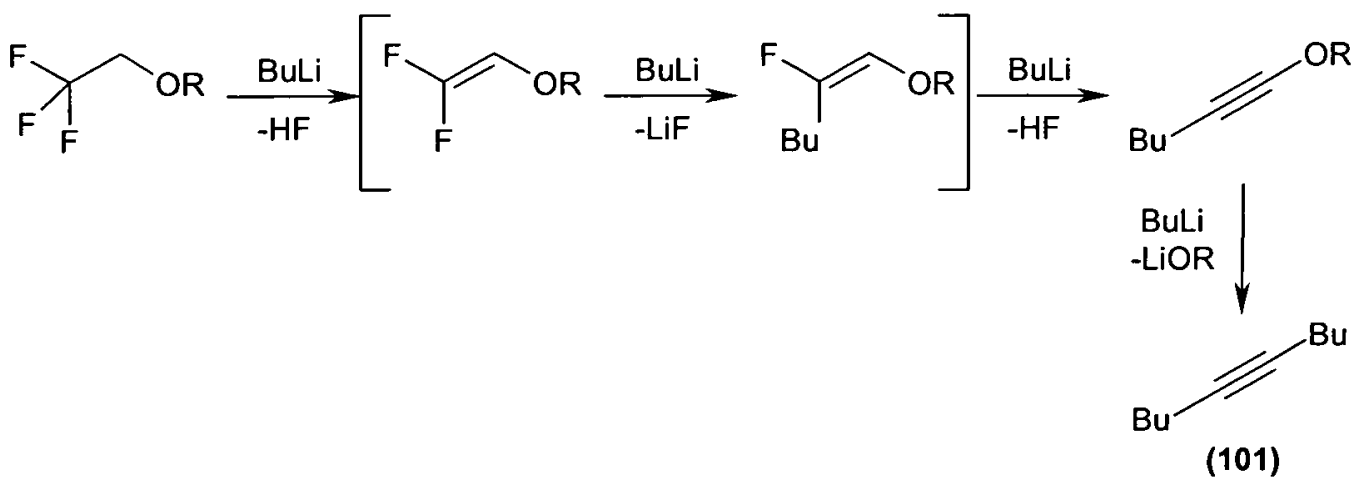

Figure 12:18: Formation of symmetrical acetylenes trifluoroethers.

Ishikawa notes some differences in reactivity between trifluoroethyl ethers and thio ethers. The ethers frequently reacted to give the symmetrical acetylenes (i.e. structure (101) above) but the thio ethers rarely did. This observation he attributed to the polarisability of the molecule. The thio ethers can spread the electron density through the molecule better than the phenyl ether which in turn is better able to stabilise the spread of charge than the alkyl ether (see Figure 12:19). This mirrors the order of reactivity of the acetylenes with the alkyl ethers being the most reactive.

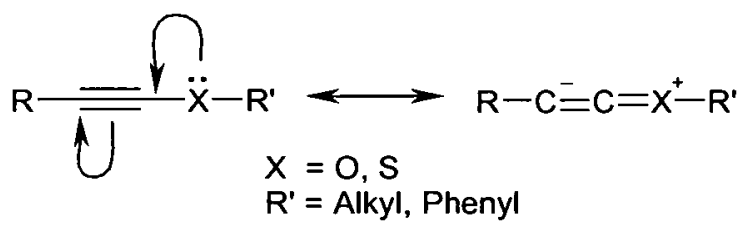

Figure 12:19: Polarisability of acetylene ethers.

If the trifluoroethyl sulfate esters undergo a similar reaction then the intermediate acetylinic sulfate would be less able to disperse the electron density of the carboncarbon triple bond than an alkyl ether and would consequently be expected to be even more reactive.

If this reaction mechanism is taking place then di-tert-butoxyethyne (102) would be the expected by-product. However such compounds are known to undergo elimination reactions in basic solution to give iso-butene and a ketene ${ }^{186}$ which would undergo an insertion reaction with the solvent to give a tert-butyl ester (see Figure 12:20). 

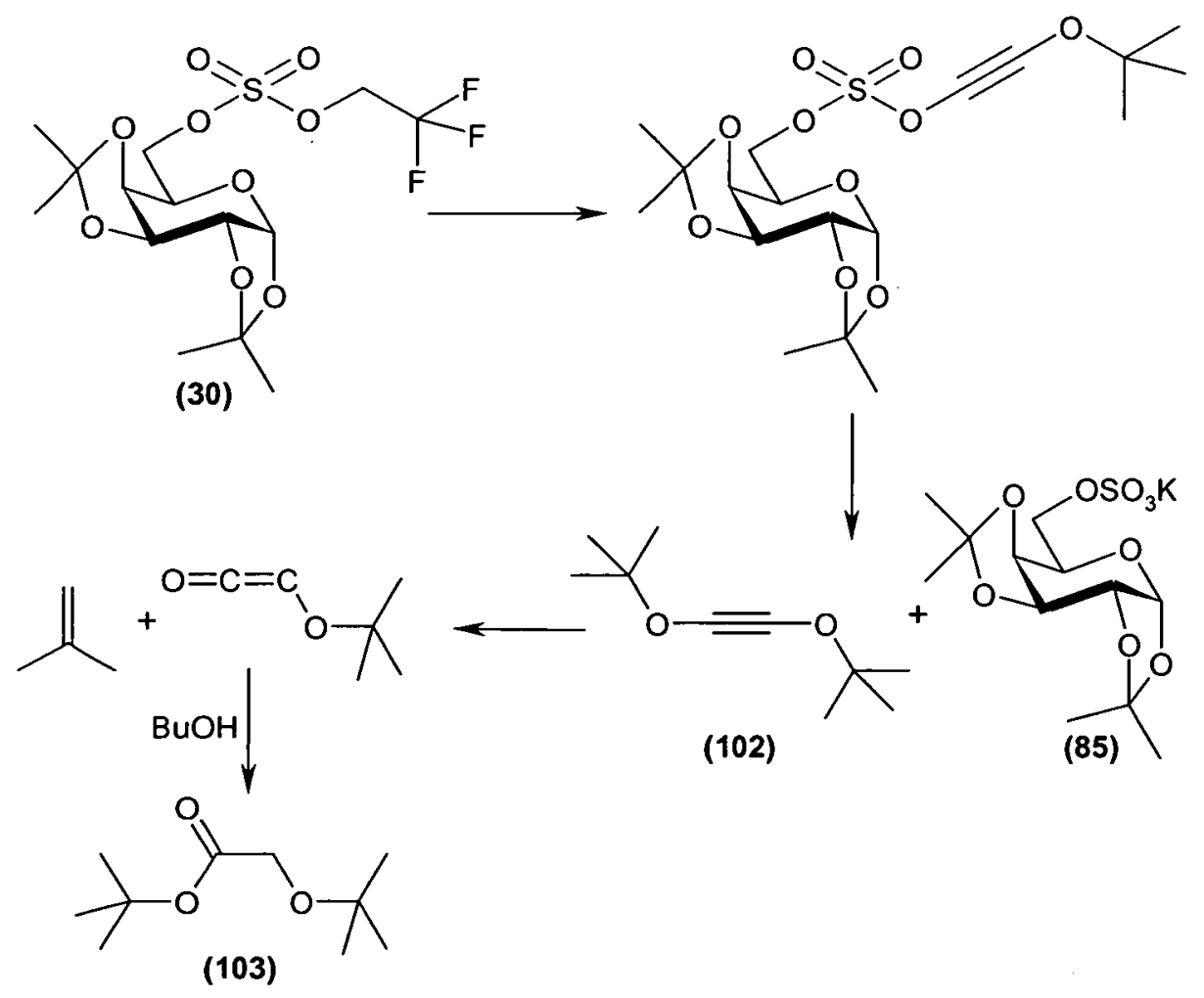

Figure 12:20: Potential mechanism.

A sample was taken from the deprotection of (30) in butanol and was examined by infra red spectrometry. No acetylene stretches could be observed. However when a solution of (30) in a 1:1 mixture of diethyl ether and hexane was treated with phenyllithium at $-78^{\circ} \mathrm{C}$ and allowed to warm to room temperature (see Figure 12:21) carbon/ carbon triple bond stretches $\left(2274 \mathrm{~cm}^{-1}\right)$ could be observed in the infra red spectra of the reaction mixture. These stretches are not associated with diphenyl ethylene (105) as symmetry inactivates this stretch in the infra red so must be due to the intermediate sugar complex (104). 


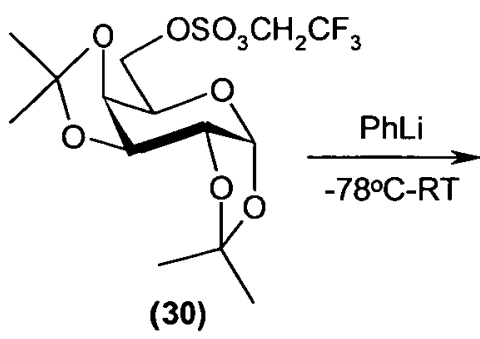

(30)
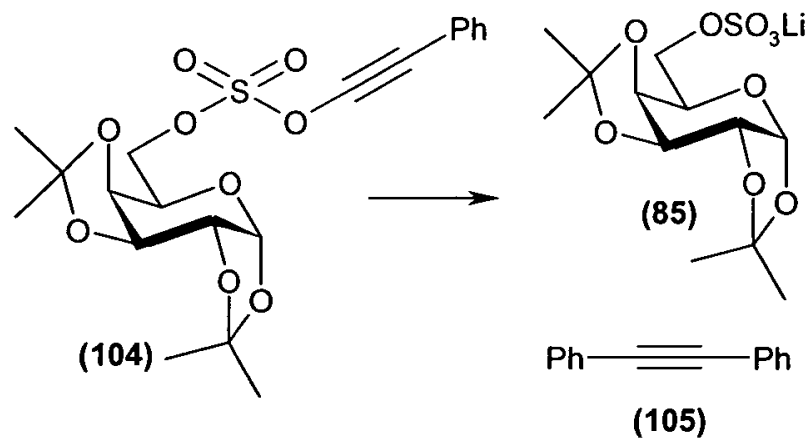

Figure 12:21: Reaction with phenyllithium.

Peaks associated with diphenyl ethyne (105) could be observed by mass spectroscopy of the crude reaction mixture $\left(\mathrm{MH}^{+} 179, \mathrm{MLi}^{+} 185\right)$ see Figure 12:22.

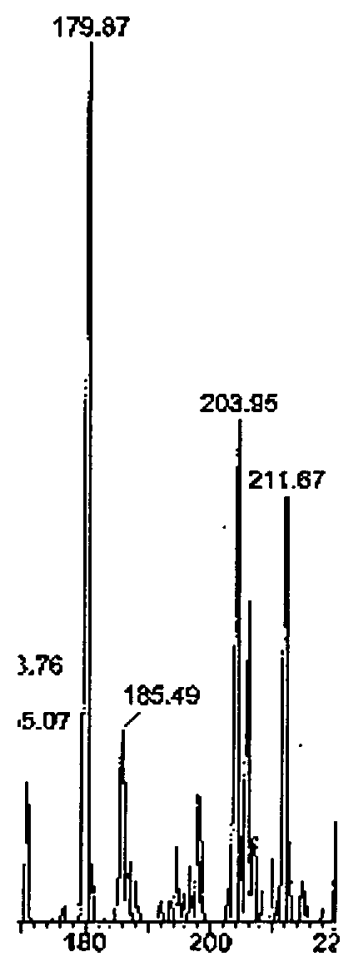

Figure 12:22: Mass Spectrum of (105)

A reaction was designed to trap any ketene formed in during the reaction. Ketenes are known to react with di-iso-propyl-carbodiimide (DIC) to form a $\beta$-lactam ${ }^{187}$ see Figure 12:23. 
<smiles>[R]OC=C=O</smiles>

Figure 12:23: $\beta$-Lactam formation.

However when (9i) was heated with di-iso-propyl-carbodiimide in the presence of potassium tert-butoxide the only isolatable product was the previously identified elimination product (96) see Figure 12:24.
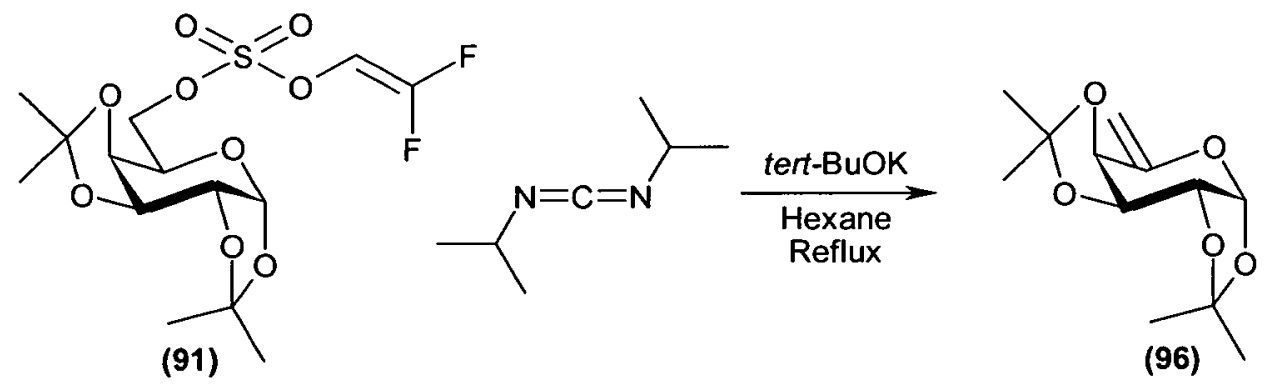

Figure 12:24: DIC elimination.

Presumably this reaction is due to the increased basisity of potassium tert-butoxide in hexane compared to tert-butanol.

Although it has not been possible to trap the postulated ketene when a sample of the deprotection reaction of (30) was analysed by a mass spectrometer it was possible to find peaks associated with the tert-butyl ester (103) (M-188) see Figure 12:25. 


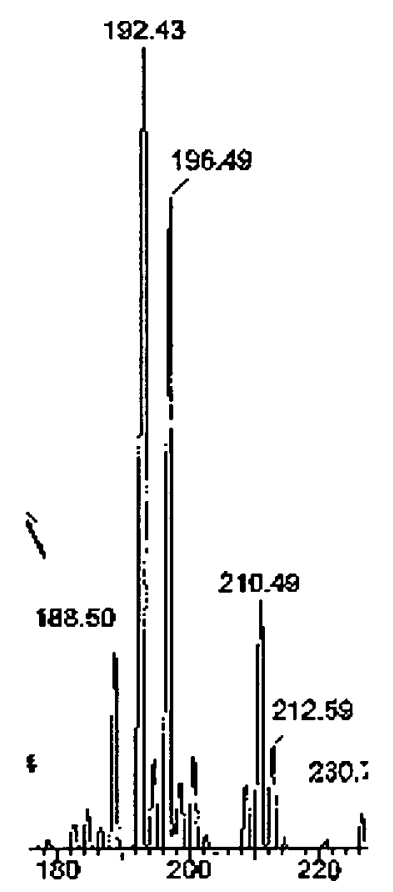

Figure 12:25: Mass Spectrum of (103).

\subsection{Conclusions.}

There is excellent evidence that the deprotection mechanism is as shown in Figure 12:20 and this also explains why it has not been possible to isolate or identify any fluorinated by-products from the reaction mixture. There are none! All the fluorine atoms in the trifluoroethyl group end up as fluoride ions (as potassium fluoride). The collapse of the dibutyl ethyne also explains the formation of iso-butene during the reaction. The mechanism also explains why altering the properties of the base has little effect on the outcome of the reaction. As the reaction depends on a combination of factors. The base must be strong enough to perform the dehydrofluorination reaction (which is probably the rate limiting step) but still have a nucleophilic property to be involved in the addition-elimination reaction and the final nucleophilic displacement.

It could be argued that using 5 equivalents of phenyllithium from $-78^{\circ} \mathrm{C}$ to room temperature is a milder procedure than heating with potassium butoxide and this 
reaction could represent a third deprotection protocol however it has not been fully explored.

\section{Conclusions.}

This research project has established, the trifluoroethyl group as a stable and versatile protecting group for sulfate monoesters of carbohydrates.

An acceptable method for the synthesis of trifluoroethyl protected sulfate monoesters has been devised and optimised. Trifluorodiazoethane has been established as highly reliable alkylating agent for sulfate monoesters across a range of challenging examples.

The trifluoroethyl group has been shown to be a stable protecting group and is resistant to a broad spectrum of chemical conditions often used to manipulate other protecting groups.

Trifluoroethyl protected sulfates have been incorporated into synthetic sequences aimed at more complex structures. While the protected sulfates generate some difficulties in such syntheses there are no problems that cannot be circumvented by adoption of an appropriate synthetic strategy.

A number of methods have been developed for the removal of the trifluoroethyl group from the sulfate monoester. The trifluoroethyl group may be removed by potassium tert-butoxide at elevated temperature, with phenyllithium by raising the temperature from $-78^{\circ} \mathrm{C}$ to room temperature, or by treatment with butyllithium at low temperature coupled with an oxidative cleavage. Unfortunately these methods all require strong bases. This is a significant disadvantage to the use of the trifluoroethyl group as a protecting group for sulfate esters. While these deprotection conditions are not ideal they do not exclude the use of the trifluoroethyl group as a protecting group 
as has been proven with a number of examples. The mechanism for the deprotection reaction with potassium tert-butoxide has been established and this knowledge may aid in the evolution of other milder removal methods.

In short this project has been successful in developing a protecting group for sulfate esters of carbohydrate. The trifluoroethyl group is introduced more easily and is superior in stability to any other sulfate protecting group published to date. While the 2,2,2-trifluoroethyl group may not be the perfect protecting group, this project has verified the original theory that sulfate diesters of highly electron withdrawing alkyl groups will be stable to nucleophilic cleavage and has established a precedent for the synthesis of such compounds. 


\section{Section Three:}

\section{Experimental.}




\section{General Experimental.}

This section contains detailed experimental information on all compounds synthesised during this project. Unless otherwise stated all reagents were available from commercial sources. All solvents used were of commercially available anhydrous grade except diethyl ether and tetrahydrofuran which were distilled from sodium benzophenone ketyl and also dichloromethane which was distilled from calcium hydride. Compounds lacking chromophores were visualised on Merck silica gel $60 \mathrm{~F}_{254}$ TLC plates using either ammonium molybdate dip (50g of ammonium molybdate tetrahydrate was dissolved in $500 \mathrm{~cm}^{3}$ of water and $27 \mathrm{~cm}^{3}$ of sulfuric acid was added. The solution was then diluted with water to $1000 \mathrm{~cm}^{3}$ ) or alkaline potassium permanganate solution (potassium permanganate $(10 \mathrm{~g})$, potassium carbonate $(50 \mathrm{~g})$ and sodium hydroxide (40 pellets) were dissolved in $1000 \mathrm{~cm}^{3}$ of water) with subsequent heating. Column chromatography was performed on BDH Silica gel for flash chromatography $40-63 \mu \mathrm{m}$. Melting points were obtained on a Gallenkamp Melting point apparatus and are uncorrected. Elemental analysis was carried out by the University of Edinburgh Microanalytical Service on a Perkin Elmer $2400 \mathrm{CHN}$ Elemental Analyser. Optical Rotations were measured using a $1 \mathrm{dm}$ path length (c given as $\mathrm{g} / 100 \mathrm{~cm}^{3}$ ) on a Optical Activity Ltd polarimeter at $16^{\circ} \mathrm{C}$. Infra red spectra were obtained from thin films of compound suspended on $3 \mathrm{M}$ Type 61 (Polyethylene) Disposable IR Cards and were recorded on a Bio Rad SPC3200 FTIR spectrometer. NMR spectra were performed in the stated solvent at room temperature and were recorded on Varian Gemini $2000\left(200 \mathrm{MHz}{ }^{1} \mathrm{H}\right)$ and Brucker AC250 $\left(250 \mathrm{MHz}{ }^{1} \mathrm{H}, 62 \mathrm{MHz}{ }^{13} \mathrm{C}\right)$ instruments. Mass spectra were obtained from a Micromass Platform LCMS (sample strength $1 \mathrm{mgml}^{-1}$, loop injection of $20 \mu 1$, solvent $50 / 50$ acetonitrile/water at $0.2 \mathrm{~cm}^{3} \mathrm{~min}^{-1}$ using the stated ionisation mode (+ve or -ve) using the stated ionisation probe (electrospray or APCI (Atmospheric Pressure Chemical Ionisation) spectrometer. Accurate mass spectra were obtained from a Kratos MS50TC instrument using FAB ionisation technique. $\mathrm{X}$ ray crystal structures 
were obtained from a Stoe Stadi-H diffractometer operating with $\mathrm{Cu}-\mathrm{K} \alpha$ radiation and equipped with an Oxford Cyrosystems 1.t. device.

\section{Experimental.}

Toluene-4-sulfonic acid 2,2,2-trichloroethyl ester (1).<smiles>Cc1ccc(S(=O)(=O)OCC(Cl)(Cl)Cl)cc1</smiles>

(1)

To a solution of para-toluenesulfonyl chloride $(5.00 \mathrm{~g}, 28 \mathrm{mmol})$ in pyridine $\left(8 \mathrm{~cm}^{3}\right)$ was added 2,2,2-trichloroethanol $(3.90 \mathrm{~g}, 26 \mathrm{mmol})$. The mixture was stirred at room temperature for $20 \mathrm{hr}$. Water $\left(15 \mathrm{~cm}^{3}\right)$ was added and the aqueous layer extracted with dichloromethane $\left(3 \times 50 \mathrm{~cm}^{3}\right)$ the combined organic layers were washed with $2 \mathrm{~N} \mathrm{HCl}$ soln. $\left(15 \mathrm{~cm}^{3}\right)$, sat. potassium bicarbonate soln. $\left(15 \mathrm{~cm}^{3}\right)$ dried $\left(\mathrm{MgSO}_{4}\right)$ and evaporated under reduced pressure. The resulting colourless solid was recrystallised in hexane to give (1) $(6.18 \mathrm{~g}, 77 \%) . \mathrm{mp} 74-77^{\circ} \mathrm{C}$ (from hexane); $v_{\max } / \mathrm{cm}^{-1} 755,861$, $1015,1193,1371,1463,2293,2847,2916 ; \delta_{\mathrm{H}}\left(200 \mathrm{MHz} ; \mathrm{CDCl}_{3}\right) 2.46\left(3 \mathrm{H}, \mathrm{s}, \mathrm{CH}_{3}\right)$, $4.53\left(2 \mathrm{H}, \mathrm{s}, \mathrm{CH}_{2} \mathrm{CCl}_{3}\right), 7.37\left(2 \mathrm{H}, \mathrm{d}, \mathrm{J}_{\text {ortho }} 8.5\right), 7.84\left(2 \mathrm{H}, \mathrm{d}, \mathrm{J}_{\text {ortho }} 8.5\right) ; \delta_{\mathrm{C}}(62 \mathrm{MHz}$; $\left.\mathrm{CDCl}_{3}\right) 21.5\left(\mathrm{CH}_{3}\right), 77.6\left(\mathrm{CH}_{2}\right), 93.0\left(\mathrm{CCl}_{3}\right), 127.9(2 \mathrm{xCH}), 129.9(2 \mathrm{xCH}), 131.9$ (Ar), 145.7 (Ar).

Toluene-4-sulfonic acid 2,2,2-trifluoroethyl ester ${ }^{188}$ (2).<smiles>Cc1ccc(S(=O)(=O)OCC(F)(F)F)cc1</smiles>

(2)

2,2,2-Trifluoroethanol $(1.46 \mathrm{~g}, 14.6 \mathrm{mmol})$ was added to a solution of paratoluenesulfonyl chloride $(2.86 \mathrm{~g}, 14.9 \mathrm{mmol})$ in pyridine $\left(5 \mathrm{~cm}^{3}\right)$. The mixture was stirred at room temperature for $20 \mathrm{hr}$. Water $\left(30 \mathrm{~cm}^{3}\right)$ was added and the aqueous layer extracted with dichloromethane $\left(3 \times 50 \mathrm{~cm}^{3}\right)$ the combined organic layers were washed 
with $2 \mathrm{~N} \mathrm{HCl}$ soln. $\left(15 \mathrm{~cm}^{3}\right)$, sat. potassium bicarbonate soln. $\left(15 \mathrm{~cm}^{3}\right)$ dried $\left(\mathrm{MgSO}_{4}\right)$ and evaporated under reduced pressure. The resulting yellow solid was recrystallised in hexane to give (2) $(2.30 \mathrm{~g}, 62 \%) . \mathrm{mp} 39-41^{\circ} \mathrm{C}$ (lit. $18941^{\circ} \mathrm{C}$.) $v_{\max } / \mathrm{cm}^{-1} 814,959$, $1038,1174,1286,1374,2847,2916 . \delta_{\mathrm{H}}\left(200 \mathrm{MHz} ; \mathrm{CDCl}_{3}\right) 2.43\left(3 \mathrm{H}, \mathrm{s}, \mathrm{CH}_{3}\right), 4.32$ $\left(2 \mathrm{H}, \mathrm{q}, \mathrm{J}_{\mathrm{H} \cdot \mathrm{F}}^{3} 8.0\right), 7.35\left(2 \mathrm{H}, \mathrm{d}, \mathrm{J}_{\text {ortho }} 8.0\right), 7.78\left(2 \mathrm{H}, \mathrm{d}, \mathrm{J}_{\text {ortho }} 8.5, \mathrm{~J}_{\text {meta }} 2.0\right) . \delta_{\mathrm{C}}(62 \mathrm{MHz}$; $\mathrm{CDCl}_{3}$ ) $21.4(\mathrm{C} 7), 64.3$ (q, Cl', $\left.\mathrm{J}_{\mathrm{C}-\mathrm{F}} 37.9\right), 121.7$ (q, C2', $\left.\mathrm{J}_{\mathrm{C}-\mathrm{F}} 277.6\right), 127.8(\mathrm{C} 2, \mathrm{C} 6)$, 130.0 (C3, C5), 131.5 (C1), 145.9 (C4). $\delta^{19} \mathrm{~F}\left(250 \mathrm{MHz} ; \mathrm{CDCl}_{3}\right)-74.23 . \mathrm{m} / \mathrm{z}$ (Electrospray) $\mathrm{MH}^{+} 255, \mathrm{C}_{9} \mathrm{H}_{9} \mathrm{~F}_{3} \mathrm{O}_{3} \mathrm{SH}^{+}$requires 255 .

Toluene-4-sulfonic acid 2-chloro-2,2-difluoroethyl ester (3).<smiles>Cc1ccc(S(=O)(=O)OCC(F)(F)Cl)cc1</smiles>

(3)

2-Chloro-2,2-difluoroethanol $(1.51 \mathrm{~g}, 12.9 \mathrm{mmol})$ was added to a solution of paratoluenesulfonyl chloride $(2.56 \mathrm{~g}, 13 \mathrm{mmol})$ in pyridine $\left(5 \mathrm{~cm}^{3}\right)$. The mixture was stirred at room temperature for $20 \mathrm{hr}$. Water $\left(15 \mathrm{~cm}^{3}\right)$ was added and the aqueous layer extracted with dichloromethane $\left(3 \times 50 \mathrm{~cm}^{3}\right)$ the combined organic layers were washed with $2 \mathrm{~N} \mathrm{HCl}$ soln. $\left(15 \mathrm{~cm}^{3}\right)$, sat. potassium bicarbonate soln. $\left(15 \mathrm{~cm}^{3}\right)$ dried $\left(\mathrm{MgSO}_{4}\right)$ and evaporated under reduced pressure. The resulting yellow solid was recrystallised in hexane to give (3) $(1.12 \mathrm{~g}, 32 \%) . \mathrm{mp} 53-54^{\circ} \mathrm{C}$ (from hexane); $v_{\max } / \mathrm{cm}^{-1} 816,1374$, $1464,2847,2916 ; \delta_{\mathrm{H}}\left(250 \mathrm{MHz} \mathrm{CDCl}_{3}\right) 2.43\left(3 \mathrm{H}, \mathrm{s}, \mathrm{CH}_{3}\right), 4.40\left(2 \mathrm{H}, \mathrm{t},{ }^{3} \mathrm{~J}_{\mathrm{H}-\mathrm{F}} 10.5\right)$, $7.35\left(2 \mathrm{H}, \mathrm{d}, \mathrm{J}_{\text {ortho }} 8.0\right), 7.78\left(2 \mathrm{H}, \mathrm{d}, \mathrm{J}_{\text {ortho }} 8.5\right) ; \delta_{\mathrm{C}}\left(62 \mathrm{MHz} ; \mathrm{CDCl}_{3}\right) 21.5(\mathrm{C} 7), 69.2(\mathrm{t}$, $\left.\mathrm{C} 1{ }^{\prime},{ }^{2} \mathrm{~J}_{\mathrm{C}-\mathrm{F}} 31.1\right), 123.9\left(\mathrm{t}, \mathrm{C} 2\right.$ ', $\left.{ }^{1} \mathrm{~J}_{\mathrm{C}-\mathrm{F}} 293.3\right), 127.8$ (C2, C6), 129.9 (C3, C5), 131.6 (C1), $145.8(\mathrm{C} 4) ; \delta_{\mathrm{F}}\left(250 \mathrm{MHz} ; \mathrm{CDCl}_{3}\right)-62.51 ; \mathrm{m} / \mathrm{z}$ (Electrospray) $288\left(\mathrm{MNH}_{4}^{+}\right)$, $\mathrm{C}_{9} \mathrm{H}_{9} \mathrm{ClF}_{2} \mathrm{O}_{3} \mathrm{SNH}_{4}^{+}$requires 288 . 


\section{2-Chloro-2,2-difluoroethanol 106 (4).}<smiles>OCC(F)(F)Cl</smiles>

(4)

Sodium borohydride $(2.64 \mathrm{~g}, 70 \mathrm{mmol})$ and methanol $\left(0.5 \mathrm{~cm}^{3}\right)$ were refluxed in diethyl ether for $1.5 \mathrm{hr}$. A solution of methyl 2-chloro-2,2-difluoroacetate in diethyl ether was added and the mixture refluxed for $24 \mathrm{hr} .2 \mathrm{~N} \mathrm{HCl}$ soln. $\left(25 \mathrm{~cm}^{3}\right)$ was added and aqueous layer was extracted with diethyl ether $\left(2 \times 50 \mathrm{~cm}^{3}\right)$. The combined organic layers were dried $\left(\mathrm{MgSO}_{4}\right)$ and the solvent evaporated under reduced pressure. The product was distilled to give (4) $(4.37 \mathrm{~g}, 54 \%)$, as a colourless liquid; bp $97^{\circ} \mathrm{C}$, (lit., $\left.19097^{\circ} \mathrm{C}\right) ; v_{\max } / \mathrm{cm}^{-1} 967,1126,1214,1471,2916,3224$ (b); $\delta_{\mathrm{H}}\left(200 \mathrm{MHz} ; \mathrm{CDCl}_{3}\right)$ $3.90\left(2 \mathrm{H}, \mathrm{t},{ }^{3} \mathrm{~J}_{\mathrm{H}-\mathrm{F}} 11.0, \mathrm{CH}_{2}\right), 4.2(1 \mathrm{H}, \mathrm{s}, \mathrm{OH}) ; \delta_{\mathrm{C}}\left(62 \mathrm{MHz}, \mathrm{CDCl}_{3}\right) 66.4\left(\mathrm{t},{ }^{2} \mathrm{~J}_{\mathrm{C}-\mathrm{F}} 29.3\right.$, $\left.\mathrm{CH}_{2} \mathrm{CF}_{2} \mathrm{Cl}\right), 127.6\left(\mathrm{t}, \mathrm{J}_{\mathrm{C}-\mathrm{F}} 293.9, \mathrm{CF}_{2} \mathrm{Cl}\right) ; \delta_{\mathrm{F}}\left(250 \mathrm{MHz} ; \mathrm{CDCl}_{3}\right)-64.66$.

Comparative study of methoxide stability, Base hydrolysis of (3), (1) and (2) ${ }^{1}$.

Sodium metal $(35 \mathrm{mg}, 1.5 \mathrm{mmol})$ was added to ${ }^{4} d$-methanol. The solution was divided into three portions and to each was added $(0.11 \mathrm{mmol})$ of (3), (1) and (2). ${ }^{1} \mathrm{H} \mathrm{nmr}$ spectra were recorded at regular intervals. Peak integrals were compared to the residual methanol signals which acted as a suitable internal standard.

Table 15:1: Hydrolysis of (3).

\begin{tabular}{|c|c|c|c|c|c|c|c|c|}
\hline Time (min) & 0 & 25 & 30 & 90 & 160 & 220 & & \\
\hline $\begin{array}{c}\text { Percentage } \\
\text { of (3) }\end{array}$ & 100 & 99.2 & 97.1 & 88.0 & 82.0 & 77.2 .0 & & \\
\hline
\end{tabular}

Table 15:2 Hydrolysis of (1).

\begin{tabular}{|c|c|c|c|c|c|c|c|c|}
\hline Time (min) & 0 & 14 & 38 & 80 & 108 & 135 & 153 & 198 \\
\hline $\begin{array}{c}\text { Percentage } \\
\text { of (1) }\end{array}$ & 100 & 95.3 & 89.2 & 82.0 & 78.5 & 74.6 & 71.8 & 70.0 \\
\hline
\end{tabular}

\footnotetext{
1 This experiment was done by a final year student, Jennifer Corning, who was working under my supervision during a short research project in a related area.
} 
Table 15:3: Hydrolysis of (2).

\begin{tabular}{|c|c|c|c|c|c|c|c|c|}
\hline Time (min) & 0 & 20 & 38 & 78 & 110 & 133 & 165 & 197 \\
\hline $\begin{array}{c}\text { Percentage } \\
\text { of (2) }\end{array}$ & 100 & 99.0 & 95.0 & 91.8 & 88.1 & 85.6 & 81.8 & 79.6 \\
\hline
\end{tabular}

\section{Comparison of stability to zinc metal/acetic acid.}

To three suspensions of zinc metal $(0.20 \mathrm{~g}, 3.0 \mathrm{mmol})$ and sodium acetate $(0.50 \mathrm{~g})$ in glacial acetic acid $\left(5 \mathrm{~cm}^{3}\right)$ were added $(0.37 \mathrm{mmol})$ portions of (3), (1) and (2). Each reaction was stirred at room temperature for $48 \mathrm{hrs}$ and monitored by TLC (eluent 1 : 4 , ether : hexane).

\section{2,2,2-Trichloroethyl 1-chlorosulfate (6).}<smiles>O=S(=O)(Cl)OCC(Cl)(Cl)Cl</smiles>

(6)

Sulfuryl chloride ( $\left.4.45 \mathrm{~g}, 2.70 \mathrm{~cm}^{3}, 33 \mathrm{mmol}\right)$, was added to diethyl ether $\left(50 \mathrm{~cm}^{3}\right)$ at $-80^{\circ} \mathrm{C}$. A solution of 2,2,2-trichoroethanol $(5.0 \mathrm{~g}, 33 \mathrm{mmol})$ and pyridine $(2.60 \mathrm{~g}, 33$ mmol) in diethyl ether $\left(50 \mathrm{~cm}^{3}\right)$ was added dropwise and the mixture was stirred at $80^{\circ} \mathrm{C}$ for $15 \mathrm{~min}$, then allowed to warm to room temperature over $1 \mathrm{~h}$. The mixture was filtered through a Celite pad to remove pyridinium hydrochloride. The solvent was evaporated under reduced pressure to yield a yellow oil. The oil was purified by column chromatography (hexane) to yield (6) $(6.09 \mathrm{~g}, 75 \%)$ as a colourless oil; $v_{\max } / \mathrm{cm}^{-1} 2900,1400,12001000 ; \delta_{\mathrm{H}}\left(250 \mathrm{MHz} ; \mathrm{CDCl}_{3}\right) 4.90(2 \mathrm{H}, \mathrm{s}) ; \delta_{\mathrm{C}}(62 \mathrm{MHz}$; $\left.\mathrm{CDCl}_{3}\right) 81.1(\mathrm{C} 1), 91.2(\mathrm{C} 2) ; \mathrm{m} / \mathrm{z}(\mathrm{FAB}) 211(\mathrm{M}-\mathrm{Cl}), 129\left(\mathrm{M}^{-\mathrm{CCl}_{3}}\right)$. 
sulfate) (8) and $1 ; 2,5 ; 6$-Di- $O$-isopropylidene- $\alpha$-D-glucofuranose 3 -(2',2'dichloroethenyl sulfate) (9).

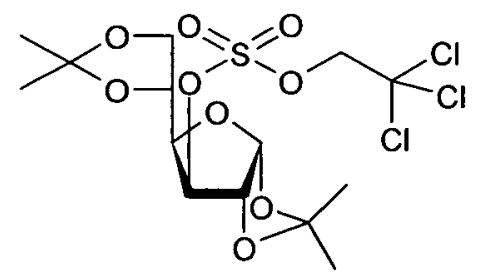

(8)

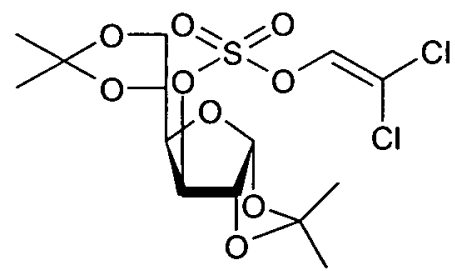

(9)

To a suspension of sodium hydride ( $60 \%$ suspension in mineral oil) $(23.0 \mathrm{mg}, 0.6$ mmol) in THF $\left(2 \mathrm{~cm}^{3}\right)$ was added a solution of 1;2,5;6-diisopropylidene- $\alpha-D-$ glucofuranose $(100 \mathrm{mg}, 0.4 \mathrm{mmol})$ in THF $\left(2 \mathrm{~cm}^{3}\right)$. After $1 \mathrm{~h}$, a solution of (6) $(248.0 \mathrm{mg}, 1.0 \mathrm{mmol})$ in THF $\left(2 \mathrm{~cm}^{3}\right)$ was added. After a further $20 \mathrm{~h}$ the reaction was filtered the solvent was evaporated under reduced pressure and the resulting colourless gel was purified by column chromatography (1:1 hexane : dichloromethane) to give $(42.0 \mathrm{mg}, 41 \%)$ of a mixture of (8) and (9). (8); $\delta_{\mathrm{H}}$ $\left(250 \mathrm{MHz} ; \mathrm{CDCl}_{3}\right) 1.32\left(3 \mathrm{H}, \mathrm{s}, \mathrm{CCH}_{3}\right), 1,34\left(3 \mathrm{H}, \mathrm{s}, \mathrm{CCH}_{3}\right), 1.43\left(3 \mathrm{H}, \mathrm{s}, \mathrm{CCH}_{3}\right), 1.50$ $\left(3 \mathrm{H}, \mathrm{s}, \mathrm{CCH}_{3}\right), 4.02-4.30(4 \mathrm{H}, \mathrm{m}), 4.77\left(1 \mathrm{H}, \mathrm{d}, \mathrm{J}_{\mathrm{a}, \mathrm{b}} 10.4, \mathrm{CH}_{2} \mathrm{CCl}_{3}\right), 4.96\left(1 \mathrm{H}, \mathrm{d}, \mathrm{J}_{2,1}\right.$ 3.5, 2-H), $5.02\left(1 \mathrm{H}, \mathrm{d}, \mathrm{J}_{3,4} 3.0,3-\mathrm{H}\right), 5.05\left(1 \mathrm{H}, \mathrm{d}, \mathrm{J}_{\mathrm{a}, \mathrm{b}} 10.5, \mathrm{CH}_{2} \mathrm{CCl}_{3}\right), 5.90(1 \mathrm{H}, \mathrm{d}$, $\left.\mathrm{J}_{1,2} 4.0,1-\mathrm{H}\right) . \mathrm{m} / \mathrm{z}$ (Electrospray) $493\left(\mathrm{M}+\mathrm{Na}^{+}\right) .(9) ; \delta_{\mathrm{H}}\left(250 \mathrm{MHz} ; \mathrm{CDCl}_{3}\right) 1.32(3 \mathrm{H}, \mathrm{s}$, $\left.\mathrm{CCH}_{3}\right), 1.34\left(3 \mathrm{H}, \mathrm{s}, \mathrm{CCH}_{3}\right), 1.43\left(3 \mathrm{H}, \mathrm{s}, \mathrm{CCH}_{3}\right), 1.50\left(3 \mathrm{H}, \mathrm{s}, \mathrm{CCH}_{3}\right), 4.02-4.30(4 \mathrm{H}$, m), $4.91\left(1 \mathrm{H}, \mathrm{d}, \mathrm{J}_{2,1}\right.$ 4.0, 2-H), $5.02\left(1 \mathrm{H}, \mathrm{d}, \mathrm{J}_{3,4}\right.$ 3.0, 3-H), $5.90\left(1 \mathrm{H}, \mathrm{d}, \mathrm{J}_{1,2} 4.0,1-\mathrm{H}\right)$, $7.21\left(1 \mathrm{H}, \mathrm{s}, \mathrm{CH}=\mathrm{CCl}_{2}\right) \cdot \mathrm{m} / \mathrm{z}$ (Electrospray) $457\left(\mathrm{M}+\mathrm{Na}^{+}\right) 459,\left(\mathrm{M}\left({ }^{37} \mathrm{Cl}\right)+\mathrm{Na}^{+}\right)$.

\section{1;2,3;4-Di- $O$-isopropylidene- $\alpha$-D-galactopyranose 6-chlorosulfate ${ }^{109}(11)$.}

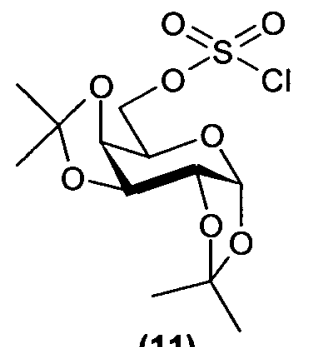

(11)

To a suspension of sodium hydride (60\% suspension in mineral oil) $(23.0 \mathrm{mg}, 0.6$ mmol) in THF $\left(2 \mathrm{~cm}^{3}\right)$ was added a solution of 1;2,3;4-diisopropylidene- $\alpha-D$ - 
galactopyranose $(100.0 \mathrm{mg}, 0.4 \mathrm{mmol})$ in THF $\left(2 \mathrm{~cm}^{3}\right)$. After $1 \mathrm{~h}$, a solution of (6) $(248.0 \mathrm{mg}, 1.0 \mathrm{mmol})$ in THF $\left(2 \mathrm{~cm}^{3}\right)$ was added. After a further $20 \mathrm{~h}$ the reaction was filtered. The solvent was evaporated under reduced pressure and the resulting colourless gel was purified by column chromatography (10:1 hexane : diethyl ether) to give (11) $(58.0 \mathrm{mg}, 32 \%)$ as a colourless solid; $\delta_{\mathrm{H}}\left(250 \mathrm{MHz} ; \mathrm{CDCl}_{3}\right) 1.19(6 \mathrm{H}, \mathrm{s}$, $\left.2 \mathrm{xCH}_{3}\right), 1.22\left(3 \mathrm{H}, \mathrm{s}, \mathrm{CH}_{3}\right), 1.28\left(3 \mathrm{H}, \mathrm{s}, \mathrm{CH}_{3}\right), 4.15(1 \mathrm{H}, \mathrm{m}, 5-\mathrm{H}), 4.25\left(1 \mathrm{H}, \mathrm{q}, \mathrm{J}_{4,5} 2.0\right.$, $\left.\mathrm{J}_{4,3} 7.5,4-\mathrm{H}\right), 4.35\left(1 \mathrm{H}, \mathrm{dd}, \mathrm{J}_{2,1} 4.5, \mathrm{~J}_{2,3} 2.5,2-\mathrm{H}\right), 4.52-4.67(3 \mathrm{H}, \mathrm{m}, 3-\mathrm{H}, 6 \mathrm{a}-\mathrm{H}, 6 \mathrm{~b}-\mathrm{H})$ $5.53\left(1 \mathrm{H}, \mathrm{d}, \mathrm{J}_{1,2} 4.5,1-\mathrm{H}\right) ; \delta_{\mathrm{C}}\left(62 \mathrm{MHz} ; \mathrm{CDCl}_{3}\right) 108.7$ and 107.5 (acetal), $95.3(\mathrm{C}-1)$, 73.0 (C-4), 69.6, 69.5 (C-2, C-3), 69.3 (C-6), 67.1 (C-5), 24.6, 23.4, 22.9, 23.0 $\left(\mathrm{CCH}_{3}\right)$.

\section{1,2,3,4-Tetra- $O$-acetyl-6-chloro-6-deoxy- $\beta$-D-glucopyranose 110 (14).}

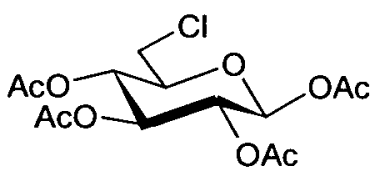

(14)

To a solution of 1,2,3,4-tetra- $O$-acetyl- $\beta$-D-glucopyranose $(110.0 \mathrm{mg}, 0.3 \mathrm{mmol}), 2,6$ dimethylaminopyridine $(50.0 \mathrm{mg}, 0.4 \mathrm{mmol})$ and pyridine $\left(1 \mathrm{~cm}^{3}\right)$ in dichloromethane $\left(2 \mathrm{~cm}^{3}\right)$ was added (6) $(785.0 \mathrm{mg}, 3.0 \mathrm{mmole})$. After $24 \mathrm{~h}$ at room temperature, $2 \mathrm{~N} \mathrm{HCl}$ soln. $\left(5 \mathrm{~cm}^{3}\right)$ was added. The mixture was extracted with dichloromethane $(3 \mathrm{x}$ $10 \mathrm{~cm}^{3}$ ) the combined organic layers were dried with $\mathrm{MgSO}_{4}$ and the solvent evaporated under reduced pressure. The resulting yellow oil was purified by column chromatography (1:1 diethyl ether : hexane) to give (14) $(34.0 \mathrm{mg}, 20 \%)$ as a colourless solid $\mathrm{mp} 113-114^{\circ} \mathrm{C}$ (from hexane); $v_{\max } / \mathrm{cm}^{-1} 2900,1780,1480,1400$, 1220 and $1100 ; \delta_{\mathrm{H}}\left(250 \mathrm{MHz} ; \mathrm{CDCl}_{3}\right) 2.00\left(3 \mathrm{H}, \mathrm{s}, \mathrm{CCH}_{3}\right), 2.02\left(3 \mathrm{H}, \mathrm{s}, \mathrm{CCH}_{3}\right), 2.04$ $\left(3 \mathrm{H}, \mathrm{s}, \mathrm{CCH}_{3}\right), 2.11\left(3 \mathrm{H}, \mathrm{s}, \mathrm{CCH}_{3}\right), 3.54\left(1 \mathrm{H}, \mathrm{q}, \mathrm{J}_{6 \mathrm{a}, 6 \mathrm{~b}} 12.0, \mathrm{~J}_{6 \mathrm{a}, \mathrm{S}} 5.0,6 \mathrm{a}-\mathrm{H}\right), 3.67(1 \mathrm{H}, \mathrm{q}$, $\left.\mathrm{J}_{6 \mathrm{~b}, 6 \mathrm{a}} 12.0, \mathrm{~J}_{6 \mathrm{~b}, 5} 3.0,6 \mathrm{~b}-\mathrm{H}\right), 3.87\left(1 \mathrm{H}\right.$, octet, $\left.\mathrm{J}_{5,6 \mathrm{a}} 5.0, \mathrm{~J}_{5,6 \mathrm{~b}} 4.0, \mathrm{~J}_{5,4} 10.0,5-\mathrm{H}\right), 5.08-5.28$ $(3 \mathrm{H}, \mathrm{m}, 4-\mathrm{H}, 3-\mathrm{H}, 2-\mathrm{H}), 5.72\left(1 \mathrm{H}, \mathrm{d}, \mathrm{J}_{1,2} 8.0,1-\mathrm{H}\right)$. 


\section{2,2,2-Trichloroethyl-sulfonyl-1'-imidazole (16).}<smiles>O=S(=O)(OCC(Cl)(Cl)Cl)n1ccnc1</smiles>

(16)

To a solution of imidazole $(274.0 \mathrm{mg}, 4 \mathrm{mmol})$ in DMF $\left(2 \mathrm{~cm}^{3}\right)$ was added (6) (100.0mg, $0.4 \mathrm{mmol})$. After $20 \mathrm{~h}$ dil. $\mathrm{HCl}$ soln. $\left(5 \mathrm{~cm}^{3}\right)$ was added and the mixture extracted with ethyl acetate $\left(3 \times 10 \mathrm{~cm}^{3}\right)$. The combined organic layers were washed with brine $\left(10 \mathrm{~cm}^{3}\right)$ and dried with $\mathrm{MgSO}_{4}$. The solvent was evaporated under reduced pressure to give a yellow oil. Column chromatography (dichloromethane) gave (16) $(72.0 \mathrm{mg}, 63 \%)$ as a colourless solid; $\mathrm{mp} 54-57^{\circ} \mathrm{C}$ (from dichloromethane); $v_{\max } / \mathrm{cm}^{-1} 3100,1410,1200,1160,1100,1050$ and $1000 ; \delta_{\mathrm{H}}\left(250 \mathrm{MHz} ; \mathrm{CDCl}_{3}\right) 4.65$ $\left(2 \mathrm{H}, \mathrm{s}, \mathrm{CH}_{2} \mathrm{CCl}_{3}\right), 7.20\left(1 \mathrm{H}, \mathrm{dd}, \mathrm{J}_{5^{\prime}, 4^{\prime}}, 1.5, \mathrm{~J}_{5^{\prime}, 2^{\prime}} 1.0,5^{\prime}-\mathrm{H}\right), 7.39\left(1 \mathrm{H}, \mathrm{dd}, \mathrm{J}_{4^{\prime}, 5^{\prime}} 1.5, \mathrm{~J}_{4^{\prime}, 2^{\prime}}\right.$ $\left.1.0,4^{\prime}-\mathrm{H}\right), 8.02\left(1 \mathrm{H}, \mathrm{dd}, \mathrm{J}_{2}, 5^{\prime}, 1.0, \mathrm{~J}_{2}, 4^{\prime}, 1.0,2^{\prime}-\mathrm{H}\right) ; \delta_{\mathrm{c}}\left(62 \mathrm{MHz} ; \mathrm{CDCl}_{3}\right) 79.9$ $\left(\mathrm{OCH}_{2} \mathrm{CCl}_{3}\right), 91.4\left(\mathrm{CCl}_{3}\right), 117.8$ (C4'), 131.5 (C3'), 136.8 (C2'); m/z (Electrospray) $279\left(\mathrm{MH}^{+}\right)$.

\section{Bis-(2,2,2-trichloroethyl) sulfate (17).}<smiles>O=S(=O)(OCC(Cl)(Cl)Cl)OCC(Cl)(Cl)Cl</smiles>

(17)

A mixture of (6) $(1.0 \mathrm{~g}, 4.0 \mathrm{mmol})$ and 2,2,2-trichloroethanol $(1.0 \mathrm{~g}, 6.7 \mathrm{mmol})$ in pyridine $\left(10 \mathrm{~cm}^{3}\right)$ was heated at $60^{\circ} \mathrm{C}$ for $2 \mathrm{~h}$. Ethyl acetate $\left(50 \mathrm{~cm}^{3}\right)$ was added and the mixture was extracted with dil. $\mathrm{HCl}$ soln. $\left(20 \mathrm{~cm}^{3}\right)$, water $\left(20 \mathrm{~cm}^{3}\right)$ and brine $\left(20 \mathrm{~cm}^{3}\right)$. The organic layer was dried with $\mathrm{MgSO}_{4}$ and the solvent evaporated under reduced pressure to give a dark orange coloured oil. Column chromatography (1:1 dichloromethane hexane) gave (17) $(0.16 \mathrm{~g}, 10 \%)$ as a colourless solid $v_{\max } / \mathrm{cm}^{-1} 3100$, $1410,1200,1160,1100,1050$ and $1000 ; \delta_{\mathrm{H}}\left(250 \mathrm{MHz} ; \mathrm{CDCl}_{3}\right) 5.05(4 \mathrm{H}, \mathrm{s}) ; \mathrm{m} / \mathrm{z}$ (electrospray) $381\left(\mathrm{MNa}^{+}\right)$. 


\section{2,2,2-Trifluoroethyl chlorosulfate (19).}

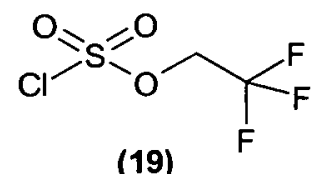

(19)

To a solution of sulfuryl chloride $(2.97 \mathrm{~g}, 22.0 \mathrm{mmol})$ in diethyl ether $\left(50 \mathrm{~cm}^{3}\right)$ at $-80^{\circ} \mathrm{C}$ was added a solution of 2,2,2-trifluoroethanol $(3.30 \mathrm{~g}, 33.0 \mathrm{mmol})$ and pyridine $(2.60 \mathrm{~g}, 33 \mathrm{mmol})$ in diethyl ether $\left(50 \mathrm{~cm}^{3}\right)$. The mixture was stirred at $-80^{\circ} \mathrm{C}$ for $1 \mathrm{~h}$ then warmed to room temperature. The reaction was filtered through Celite and the product purified by distillation to give (19) $(2.95 \mathrm{~g}, 44 \%)$ as a colourless oil bp 115 $118^{\circ} \mathrm{C}$ at $720 \mathrm{mmHg}$ (lit., $191116-118^{\circ} \mathrm{C}$ ); $v_{\max } / \mathrm{cm}^{-1} 1440,1410,1285,1180,1042 ; \delta_{\mathrm{H}}$ $\left(250 \mathrm{MHz} ; \mathrm{CDCl}_{3}\right) 4.47\left(2 \mathrm{H}, \mathrm{q}, \mathrm{J}_{\mathrm{H}, \mathrm{F}} 8.0, \mathrm{CH}_{2} \mathrm{CF}_{3}\right) ; \delta_{\mathrm{C}}\left(62 \mathrm{MHz} ; \mathrm{CDCl}_{3}\right) 68.4(\mathrm{q}, \mathrm{J} 39.4$, $\left.\mathrm{CH}_{2} \mathrm{CF}_{3}\right), 120.8\left(\mathrm{q}, \mathrm{J} 277.6, \mathrm{CF}_{3}\right) ; \delta_{\mathrm{F}}\left(250 \mathrm{MHz} ; \mathrm{CDCl}_{3}\right)-73.77 ; \mathrm{m} / \mathrm{z}$ (Electrospray) $199\left(\mathrm{MH}^{+}\right)$.

\section{1;2,5;6-Di-O-isopropylidene- $\alpha$-D-glucofuranose 3-(2',2',2'-trifluoroethyl sulfate)} (20).

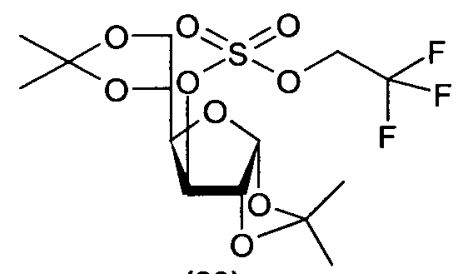

(20)

\section{Preparation of (20); Single step synthesis.}

To a suspension of sodium hydride $(23 \mathrm{mg}$ of a $60 \%$ suspension in mineral oil, $0.60 \mathrm{mmol})$ in THF $\left(2 \mathrm{~cm}^{3}\right)$ was added a solution of $1 ; 2,5 ; 6-\mathrm{di}-O$-isopropylidene- $\alpha$-Dglucofuranose in THF $\left(1 \mathrm{~cm}^{3}\right)$. The mixture was cooled to $0^{\circ} \mathrm{C}$ before the addition of (19) $(153 \mathrm{mg}, 1.20 \mathrm{mmol})$. The mixture was warmed slowly to room temperature. Water $\left(5 \mathrm{~cm}^{3}\right)$ was then added to the reaction and the product extracted with diethyl ether $\left(3 \times 5 \mathrm{~cm}^{3}\right)$. The organic layers were dried with $\mathrm{MgSO}_{4}$ and the solvent evaporated under reduced pressure. The product was purified by column chromatography $(1: 1$, dichloromethane: hexane) to give (20) $(114 \mathrm{mg}, 70 \%)$ as a colourless solid. 


\section{Preparation of (20); Two step synthesis.}

Sulfur trioxide/ pyridine complex $(473.0 \mathrm{mg}, 3.0 \mathrm{mmol})$ was added to a solution of $1 ; 2,5 ; 6$-di-O-isopropylidene- $\alpha$-D-glucofuranose $(156.0 \mathrm{mg}, 0.60 \mathrm{mmol})$ in acetonitrile $\left(10 \mathrm{~cm}^{3}\right)$. The mixture was heated at $80^{\circ} \mathrm{C}$ for $1 \mathrm{~h}$, then cooled to room temperature. Diazo solution (24) $\left(20 \mathrm{~cm}^{3}\right)$ and citric acid monohydrate $(1 \mathrm{~g})$ were added and the reaction stirred for a further $16 \mathrm{~h}$. The solvent was evaporated under reduced pressure and the residue dissolved in water $\left(20 \mathrm{~cm}^{3}\right)$. The solution was extracted with diethyl ether $\left(3 \times 50 \mathrm{~cm}^{3}\right)$, the organic layers were combined, dried with $\mathrm{MgSO}_{4}$ and the solvent evaporated under reduced pressure to give a yellow oil. The product was purified by column chromatography (dichloromethane) to give (20) $(134 \mathrm{mg}, 51 \%)$ as a colourless solid; $\mathrm{mp} 66-68^{\circ} \mathrm{C}$ (from dichloromethane); Found: C, 39.35; H, 5.0. $\mathrm{C}_{14} \mathrm{H}_{21} \mathrm{~F}_{3} \mathrm{O}_{9} \mathrm{~S}$ requires $\mathrm{C}, 39.7 ; \mathrm{H}, 5.2 \% ; v_{\max } / \mathrm{cm}^{-1} 3008,1493,1240,840 ; \delta_{\mathrm{H}}$ $\left(250 \mathrm{MHz} ; \mathrm{CDCl}_{3}\right) 1.31\left(\mathrm{CH}_{3}\right), 1.32\left(\mathrm{CH}_{3}\right), 1.41\left(\mathrm{CH}_{3}\right), 1.50\left(\mathrm{CH}_{3}\right), 4.05\left(1 \mathrm{H}, \mathrm{dd}, \mathrm{J}_{6 \mathrm{a}-6 \mathrm{~b}}\right.$ $\left.9.0 \mathrm{~J}_{6 \mathrm{a}-5} 4.0,6 \mathrm{a}-\mathrm{H}\right), 4.11\left(1 \mathrm{H}, \mathrm{dd}, \mathrm{J}_{4-3} 3.0 \mathrm{~J}_{4-5} 1.5,4-\mathrm{H}\right), 4.16\left(1 \mathrm{H}, \mathrm{dd}, \mathrm{J}_{6 \mathrm{~b}-6 \mathrm{a}} 9.0 \mathrm{~J}_{6 \mathrm{~b}-5} 5.0\right.$, $6 \mathrm{~b}-\mathrm{H}), 4.22\left(1 \mathrm{H}, \mathrm{ddd}, \mathrm{J}_{\mathrm{5}-6 \mathrm{~b}} 5.0 \mathrm{~J}_{5-6 \mathrm{a}} 4.0 \mathrm{~J}_{5-4} 1.5,5-\mathrm{H}\right), 4.55\left(1 \mathrm{H}, \mathrm{dq}, \mathrm{J}_{\mathrm{Ha}-\mathrm{Hb}} 11.0, \mathrm{~J}_{\mathrm{H}-\mathrm{F}} 8.0\right.$, $\left.\mathrm{CH}_{2} \mathrm{CF}_{3}\right), 4.82\left(1 \mathrm{H}, \mathrm{dq}, \mathrm{J}_{\mathrm{Hb}-\mathrm{Ha}} 11.0, \mathrm{~J}_{\mathrm{H}-\mathrm{F}} 8.0, \mathrm{CH}_{2} \mathrm{CF}_{3}\right), 4.91\left(1 \mathrm{H}, \mathrm{d}, \mathrm{J}_{2-1} 4.0,2-\mathrm{H}\right), 4.96$ $\left(1 \mathrm{H}, \mathrm{d}, \mathrm{J}_{3-4} 3.0,3-\mathrm{H}\right), 5.93\left(1 \mathrm{H}, \mathrm{d}, \mathrm{J}_{1-2} 4.0,1-\mathrm{H}\right) ; \delta_{\mathrm{C}}\left(62 \mathrm{MHz} ; \mathrm{CDCl}_{3}\right) 24.8\left(\mathrm{CH}_{3}\right), 26.0$ $\left(\mathrm{CH}_{3}\right), 6.4\left(\mathrm{CH}_{3}\right), 26.7\left(\mathrm{CH}_{3}\right), 66.8\left(\mathrm{q},{ }^{2} \mathrm{~J}_{\mathrm{C}-\mathrm{F}} 38.6, \mathrm{CH}_{2} \mathrm{CF}_{3}\right), 37.3(6-\mathrm{C}), 71.5(\mathrm{CH})$, $79.3(\mathrm{CH}), 82.4(\mathrm{CH}), 85.5(\mathrm{CH}), 104.8(1-\mathrm{C}), 109.9\left(\mathrm{CH}_{3}-\mathrm{C}-\mathrm{CH}_{3}\right), 112.7\left(\mathrm{CH}_{3}-\mathrm{C}-\right.$ $\left.\mathrm{CH}_{3}\right), 121.4\left(\mathrm{q}, \mathrm{J}_{\mathrm{C}-\mathrm{F}} 277.2, \mathrm{CF}_{3}\right) ; \delta_{\mathrm{F}}\left(250 \mathrm{MHz} ; \mathrm{CDCl}_{3}\right)-73.9 ; \mathrm{m} / \mathrm{z}(\mathrm{FAB}) 423\left(\mathrm{MH}^{+}\right)$, $\mathrm{C}_{12} \mathrm{H}_{22} \mathrm{~F}_{3} \mathrm{O}_{9} \mathrm{~S}$ requires 423.09367 found 423.09308 (dev. -1.38).

\section{Bis-(2,2,2-trichloroethyl) sulfite ${ }^{114}$ (21).}<smiles>O=S(OCC(Cl)(Cl)Cl)OCC(Cl)(Cl)Cl</smiles>

(21)

Thionyl chloride $(3.95 \mathrm{~g}, 33.0 \mathrm{mmol})$ was added to 2,2,2-trichloroethanol $(10.0 \mathrm{~g}$, $67.0 \mathrm{mmol}$ ). The mixture was heated at $50^{\circ} \mathrm{C}$ for $1 \mathrm{~h}$ (until no more $\mathrm{HCl}$ is evolved). Excess reagents were removed by vacuum distillation $\left(60^{\circ} \mathrm{C}\right.$ at $\left.25 \mathrm{mmHg}\right)$ leaving (21) $(7.39 \mathrm{~g}, 65 \%)$ as a yellow oil; $v_{\max } / \mathrm{cm}^{-1} 825,982,1228,1472,2916 ; \delta_{\mathrm{H}}$ 
$\left(250 \mathrm{MHz} ; \mathrm{CDCl}_{3}\right) 4.49\left(2 \mathrm{H}, \mathrm{d}, \mathrm{J}_{\mathrm{H} \cdot \mathrm{H}} 12.0, \mathrm{CH}_{2} \mathrm{CCl}_{3}\right), 4.65\left(2 \mathrm{H}, \mathrm{d}, \mathrm{J}_{\mathrm{H}-\mathrm{H}} 12.0, \mathrm{CH}_{2} \mathrm{CCl}_{3}\right)$; $\delta_{\mathrm{C}}\left(62 \mathrm{MHz} ; \mathrm{CDCl}_{3}\right) 72.1\left(\mathrm{CH}_{2}\right), 94.2\left(\mathrm{CCl}_{3}\right)$.

\section{Standard solution of 2,2,2-trifluorodiazoethane $\mathrm{e}^{117,124}$ (24).}

To a solution of 2,2,2-trifluoroethylamine hydrochloride $(5.40 \mathrm{~g}, 40 \mathrm{mmol})$ in water $\left(20 \mathrm{~cm}^{3}\right)$ at $0^{\circ} \mathrm{C}$ was added a solution of sodium nitrite $(3.00 \mathrm{~g}, 44 \mathrm{mmol})$ in water $\left(10 \mathrm{~cm}^{3}\right)$. A stream of nitrogen gas is bubbled through the solution. The glass was passed through a vessel containing potassium hydroxide (2g approx.) pellets into a flask of acetonitrile $\left(40 \mathrm{~cm}^{3}\right)$ which has been cooled to $-40^{\circ} \mathrm{C}$. The aqueous solution is kept at $0^{\circ} \mathrm{C}$ for $1 \mathrm{~h}$ then was warmed to room temperature for a further $1 \mathrm{~h}$. The product (24) was collected as a solution in acetonitrile and was used without further purification.

Trifluoroacetyltriphenylsilane 2,4,6-triisopropylbenzenesulfonylhydrazone (25).

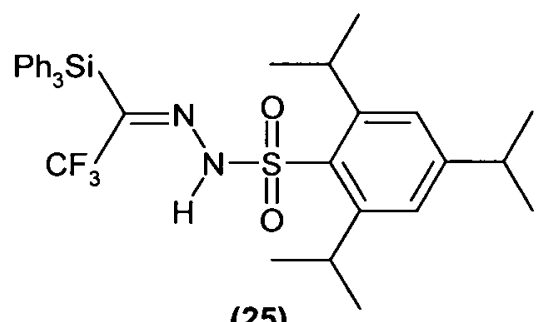

(25)

A mixture of (26) $(0.89 \mathrm{~g}, 2.5 \mathrm{mmol})$ and 2,4,6-triisopropylbenzenesulfonylhydrazine $(0.75 \mathrm{~g}, 2.5 \mathrm{mmol})$ in acetonitrile $\left(30 \mathrm{~cm}^{3}\right)$ was stirred at room temperature for $3 \mathrm{~h}$. The mixture was then extracted with hexane $\left(4 \times 100 \mathrm{~cm}^{3}\right)$, the hexane extracts were evaporated under reduced pressure to give a yellow oil. The oil was purified by column chromatography (1:1 dichloromethane : hexane) to give (25) $(0.69 \mathrm{~g}, 41 \%)$ as a yellow solid; mp 154-156 ${ }^{\circ} \mathrm{C}$ (from hexane) (lit., 118 158-160); 3529, 2916, 1464, $1426,1267,1139,1111,925,716,697 ; \delta_{\mathrm{H}}\left(250 \mathrm{MHz} ; \mathrm{CDCl}_{3}\right) 1.05(12 \mathrm{H}, \mathrm{d}, \mathrm{J} 7.0)$, 1.28 (6H, d, J 7.0), 2.92 (1H, hept., J 7.0), 3.93 (2H, hept., 7.0), 7.14-7.64 (17H, m), $9.33(1 \mathrm{H}, \mathrm{s}, \mathrm{NH}) ; \delta_{\mathrm{C}}\left(62 \mathrm{MHz} ; \mathrm{CDCl}_{3}\right) 23.3\left(2 \times \mathrm{CH}_{3}\right), 24.4\left(4 \times \mathrm{CH}_{3}\right), 29.9(2 \times \mathrm{CH})$, $34.0(\mathrm{CH}), 119.6$ (q, $\left.\mathrm{J}_{\mathrm{C}-\mathrm{F}} 286.7, \mathrm{CF}_{3}\right), 123.7$ (3 x CH arom.), 127.7 (6 $\mathrm{x} \mathrm{CH}$ Arom.), 128.1 ( $2 \times \mathrm{CH}$ Arom.), 21.9 (3 x CH Arom.), $135.7\left(\mathrm{SO}_{2}-\mathrm{C}\right.$ Arom.), 135.9 (6 x $\mathrm{CH}$ 
Arom.), $142.0\left(\mathrm{q},{ }^{2} \mathrm{~J}_{\mathrm{C}-\mathrm{F}} 28.8, C(=\mathrm{N}) \mathrm{CF}_{3}\right), 151.4$ (2 x C Arom.), 153.7 (1 x C Arom.); $\delta_{\mathrm{F}}\left(250 \mathrm{MHz} ; \mathrm{CDCl}_{3}\right)-60.83 ; \mathrm{m} / \mathrm{z}$ (Electrospray) $659\left(\mathrm{MNa}^{+}\right)$.

\section{Trifluoroacetyl triphenylsilane $\mathrm{121}^{126)}$.}<smiles>O=C([Hg]c1ccccc1)C(F)(F)F</smiles>

(26)

To a suspension of finely cut pieces of lithium metal $(0.60 \mathrm{~g}, 20 \mathrm{mmol})$ in THF $\left(20 \mathrm{~cm}^{3}\right)$ was added a solution of triphenyl chlorosilane $(5.90 \mathrm{~g}, 20 \mathrm{mmol})$. The resulting grey suspension was stirred for $3 \mathrm{~h}$ over which time the suspension turned black. The suspension was then added to a suspension of anhydrous copper iodide $(3.80 \mathrm{~g}, 20 \mathrm{mmol})$ in THF $\left(10 \mathrm{~cm}^{3}\right)$ at $-50^{\circ} \mathrm{C}$. The mixture was stirred at $-50^{\circ} \mathrm{C}$ for $1 \mathrm{~h}$ before the addition of trifluoroacetic anhydride. The mixture was stirred at $-50^{\circ} \mathrm{C}$ for a further $1 \mathrm{~h}$ and then at room temperature for $16 \mathrm{~h}$. A solution of $(10 \%)$ sulfuric acid was then added $\left(75 \mathrm{~cm}^{3}\right)$ and the mixture filtered. The filtrate was extracted with dichloromethane $\left(3 \times 100 \mathrm{~cm}^{3}\right)$ the combined organic layers were dried with $\mathrm{MgSO}_{4}$ and the solvent evaporated under reduced pressure. The resulting solid was recrystallised from hexane to give (26) $(4.03 \mathrm{~g}, 57 \%)$ as a yellow solid; $\mathrm{mp} 119$ $122^{\circ} \mathrm{C}$ (from hexane), (lit., 121 122); $v_{\max } / \mathrm{cm}^{-1} 2916,1558,1464,1170,738 ; \delta_{\mathrm{H}}$ $\left(250 \mathrm{MHz} ; \mathrm{CDCl}_{3}\right)$ 7.40-7.63 (m, Arom.); $\delta_{\mathrm{C}}\left(62 \mathrm{MHz} ; \mathrm{CDCl}_{3}\right) 115.5$ (q, $\mathrm{J}_{\mathrm{C}-\mathrm{F}} 296.5$, $\mathrm{CF}_{3}$ ), 128.3 (9 x C Arom.), 130.9 (3 x Arom.), 136.1 (6 x C Arom.), 220.1 (q, ${ }^{2} \mathrm{~J}_{\mathrm{C}-\mathrm{F}}$ 35.4, $\left.C=\mathrm{OCF}_{3}\right) ; \delta_{\mathrm{F}}\left(250 \mathrm{MHz} ; \mathrm{CDCl}_{3}\right)-78.55 ; \mathrm{m} / \mathrm{z}$ (Electrospray) $357\left(\mathrm{MH}^{+}\right)$.

1;2,5;6-Di- $O$-isopropylidene-6-sulfo- $\alpha$-D-glucofuranose, potassium salt ${ }^{63}$ (22).

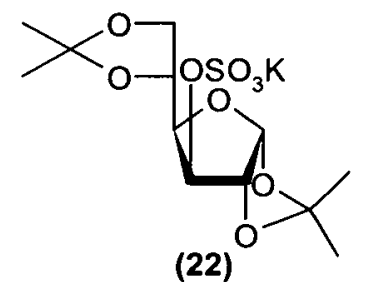

To a solution of $1 ; 2,5 ; 6$-di- $O$-isopropylidene- $\alpha$-D-glucofuranose $(1.0 \mathrm{~g}, 3.85 \mathrm{mmol})$ in pyridine $\left(10 \mathrm{~cm}^{3}\right)$ at $-15^{\circ} \mathrm{C}$ was added a solution of chlorosulfonic acid $(0.45 \mathrm{~g}$, 
$3.85 \mathrm{mmol})$ in dichloromethane $\left(5 \mathrm{~cm}^{3}\right)$ such that a temperature of $-10^{\circ} \mathrm{C}$ was maintained. The mixture was stirred at room temperature for $16 \mathrm{~h}$ before the addition of water $\left(50 \mathrm{~cm}^{3}\right)$ The mixture was then evaporated under reduced pressure and the resulting oil dissolved in water $\left(20 \mathrm{~cm}^{3}\right)$. The aqueous mixture was extracted with diethyl ether $\left(3 \times 20 \mathrm{~cm}^{3}\right)$ and the aqueous layer was then passed down a column of Dowex 50 ion-exchange resin $\left(\mathrm{K}^{+}\right.$form) evaporation under reduced pressure gave a white solid which was purified by column chromatography (4:1 dichloromethane : methanol) to give (22) $(0.57 \mathrm{~g}, 40 \%)$ as a white solid; mp $157-159^{\circ} \mathrm{C}$ (from water) (lit., $\left.{ }^{65} 159-163^{\circ} \mathrm{C}\right) ; v_{\max } / \mathrm{cm}^{-1} 3030,1240,840 ; \delta_{\mathrm{H}}\left(250 \mathrm{MHz} ; \mathrm{D}_{2} \mathrm{O}\right) 1.22\left(3 \mathrm{H}, \mathrm{s}, \mathrm{CH}_{3}\right)$, $1.23\left(3 \mathrm{H}, \mathrm{s}, \mathrm{CH}_{3}\right), 1.31\left(3 \mathrm{H}, \mathrm{s}, \mathrm{CH}_{3}\right), 1.38\left(3 \mathrm{H}, \mathrm{s}, \mathrm{CH}_{3}\right), 3.89-3.99(2 \mathrm{H}, \mathrm{m}, 4-\mathrm{H}, 5-\mathrm{H})$, 4.35-4.40 (2H, m, 6a-H, 6b-H), $4.68(1 \mathrm{H}, \mathrm{d}, \mathrm{J} 2.5,3-\mathrm{H}), 4.83\left(1 \mathrm{H}, \mathrm{d}, \mathrm{J}_{2,1} 3.5,2-\mathrm{H}\right)$, $5.94\left(1 \mathrm{H}, \mathrm{d}, \mathrm{J}_{1,2} 3.5,1-\mathrm{H}\right) ; \mathrm{m} / \mathrm{z}$ (-ve Electrospray) $339\left(\mathrm{M}^{-}\right)$.

\section{1,2,3,4-Tetra- $O$-acetyl- $\beta$-D-glucopyranose 6-(2',2',2'-trifluoroethyl sulfate) (29).}

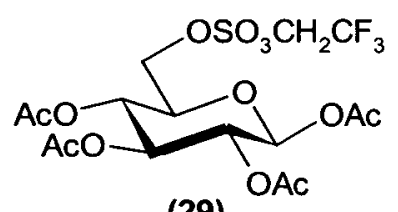

(29)

Sulfur trioxide/ pyridine complex $(473.0 \mathrm{mg}, 3.0 \mathrm{mmol})$ was added to a solution of $1,2,3,4$-tetra- $O$-acetyl- $\beta$-D-glucopyranose $(209.0 \mathrm{mg}, 0.60 \mathrm{mmol})$ in acetonitrile $\left(10 \mathrm{~cm}^{3}\right)$. The mixture was heated at $80^{\circ} \mathrm{C}$ for $1 \mathrm{~h}$, then cooled to room temperature. Diazo solution (24) $\left(20 \mathrm{~cm}^{3}\right)$ and citric acid monohydrate $(1 \mathrm{~g})$ were added and the reaction stirred for a further $16 \mathrm{~h}$. The solvent was evaporated under reduced pressure and the residue dissolved in water $\left(20 \mathrm{~cm}^{3}\right)$. The solution was extracted with diethyl ether $\left(3 \times 50 \mathrm{~cm}^{3}\right)$, the organic layers were combined, dried with $\mathrm{MgSO}_{4}$ and the solvent evaporated under reduced pressure to give a yellow oil. The product was purified by column chromatography (dichloromethane) to give (29) $(270.0 \mathrm{mg}, 93 \%)$ as a colourless solid; mp $125-129^{\circ} \mathrm{C}$ (from dichloromethane); (Found: C, 37.6; $\mathrm{H}$, 4.2. $\mathrm{C}_{16} \mathrm{H}_{21} \mathrm{~F}_{3} \mathrm{O}_{13} \mathrm{~S}$ requires $\left.\mathrm{C}, 37.65 ; \mathrm{H}, 4.1 \%\right) ; v_{\max } / \mathrm{cm}^{-1} 1240,1750,2930 ; \delta_{\mathrm{H}}$ $\left(250 \mathrm{MHz} ; \mathrm{CDCl}_{3}\right) 1.99(3 \mathrm{H}, \mathrm{s}, \mathrm{OAc}), 2.01$ (3H, s, OAc), $2.04(3 \mathrm{H}, \mathrm{s}, \mathrm{OAc}), 2.07$ $(3 \mathrm{H}, \mathrm{s}, \mathrm{OAc}), 3.93\left(1 \mathrm{H}, \mathrm{ddd}, \mathrm{J}_{5-4} 10.0, \mathrm{~J}_{5-6 \mathrm{a}} 5.0, \mathrm{~J}_{5-6 \mathrm{~b}} 2.5,5-\mathrm{H}\right), 4.28\left(1 \mathrm{H}, \mathrm{dd}, \mathrm{J}_{6 \mathrm{a}-6 \mathrm{~b}} 11.0\right.$, 
$\left.\mathrm{J}_{6 \mathrm{a}-5} 5.0,6 \mathrm{a}-\mathrm{H}\right), 4.38\left(1 \mathrm{H}, \mathrm{dd}, \mathrm{J}_{6 \mathrm{~b}-6 \mathrm{a}} 11.0, \mathrm{~J}_{6 \mathrm{~b}-5} 2.5,6 \mathrm{~b}-\mathrm{H}\right), 4.50\left(2 \mathrm{H}, \mathrm{qd}, \mathrm{J}_{\mathrm{H}-\mathrm{F}} 8.0, \mathrm{~J}_{\mathrm{H}-\mathrm{H}}\right.$ $\left.1.0, \mathrm{CH}_{2} \mathrm{CF}_{3}\right), 5.05\left(1 \mathrm{H}, \mathrm{dd}, \mathrm{J}_{4-5} 10.0, \mathrm{~J}_{4-3} 9.0,4-\mathrm{H}\right), 5.10\left(1 \mathrm{H}, \mathrm{dd}, \mathrm{J}_{2-3} 9.0, \mathrm{~J}_{2-1} 8.0,2-\right.$ $\mathrm{H}), 5.25\left(1 \mathrm{H}, \mathrm{dd}, \mathrm{J}_{3-2} 9.0, \mathrm{~J}_{3-4} 9.0,3-\mathrm{H}\right), 5.67\left(1 \mathrm{H}, \mathrm{d}, \mathrm{J}_{1-2} 8.0,1-\mathrm{H}\right) ; \delta_{\mathrm{C}}(62 \mathrm{MHz}$; $\left.\mathrm{CDCl}_{3}\right) 20.3\left(3 \mathrm{xCH}_{3}\right), 20.4\left(\mathrm{CH}_{3}\right), 66.7\left(\mathrm{q},{ }^{2} \mathrm{~J}_{\mathrm{C}-\mathrm{F}} 38.4, \mathrm{CH}_{2} \mathrm{CF}_{3}\right), 67.3(\mathrm{CH}), 69.6(\mathrm{CH})$, $70.4(6-\mathrm{C}), 71.5(\mathrm{CH}), 91.3(1-\mathrm{C}), 121.4\left(\mathrm{q}, \mathrm{J}_{\mathrm{C}-\mathrm{F}} 277.4, \mathrm{CF}_{3}\right), 168.8(\mathrm{C}=\mathrm{O}), 168.9$ $(\mathrm{C}=\mathrm{O}), 169.3(\mathrm{C}=\mathrm{O}), 169.8(\mathrm{C}=\mathrm{O}) ; \delta_{\mathrm{F}}\left(250 \mathrm{MHz}^{\circ} \mathrm{CDCl}_{3}\right)-74.13 ; \mathrm{m} / \mathrm{z}(\mathrm{FAB}) 451(\mathrm{M}-$ $\mathrm{OAc})$.

$1 ; 2,3 ; 4-D i-O$-isopropylidene- $\alpha$-D-galactopyranose

6-(2',2',2'-trifluoroethyl sulfate) (30).

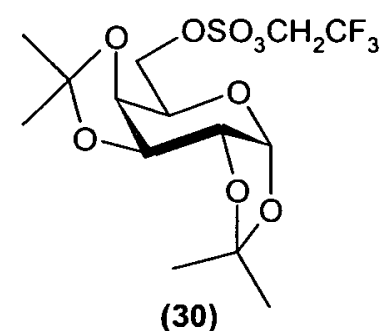

Sulfur trioxide/ pyridine complex $(473.0 \mathrm{mg}, 3.0 \mathrm{mmol})$ was added to a solution of $1 ; 2,3 ; 4$-di- $O$-isopropylidene- $\alpha$-D-galactopyranose $\quad(156.0 \mathrm{mg}, \quad 0.60 \mathrm{mmol})$ in acetonitrile $\left(10 \mathrm{~cm}^{3}\right)$. The mixture was heated at $80^{\circ} \mathrm{C}$ for $1 \mathrm{~h}$, then cooled to room temperature. Diazo solution (24) $\left(20 \mathrm{~cm}^{3}\right)$ and citric acid monohydrate $(1 \mathrm{~g})$ were added and the reaction stirred for a further $16 \mathrm{~h}$. The solvent was evaporated under reduced pressure and the residue dissolved in water $\left(20 \mathrm{~cm}^{3}\right)$. The solution was extracted with diethyl ether $\left(3 \times 50 \mathrm{~cm}^{3}\right)$, the organic layers were combined, dried with $\mathrm{MgSO}_{4}$ and the solvent evaporated under reduced pressure to give a yellow oil. The product was purified by column chromatography (dichloromethane) to give (30) $(201.0 \mathrm{mg}, 80 \%)$ as a colourless oil; $v_{\max } / \mathrm{cm}^{-1} 2943,1457,1283,1009,840 ; \delta_{\mathrm{H}}$ $\left(250 \mathrm{MHz} ; \mathrm{CDCl}_{3}\right) 1.31\left(3 \mathrm{H}, \mathrm{s}, \mathrm{CH}_{3}\right), 1.32\left(3 \mathrm{H}, \mathrm{s}, \mathrm{CH}_{3}\right), 1.42\left(3 \mathrm{H}, \mathrm{s}, \mathrm{CH}_{3}\right), 1.50(3 \mathrm{H}$, $\left.\mathrm{s}, \mathrm{CH}_{3}\right), 4.12\left(1 \mathrm{H}, \mathrm{td}, \mathrm{J}_{5-6 \mathrm{a}} 6.0, \mathrm{~J}_{5-6 \mathrm{~b}} 6.0, \mathrm{~J}_{5-4} 2.0,5-\mathrm{H}\right), 4.22\left(1 \mathrm{H}, \mathrm{dd}, \mathrm{J}_{4-3} 7.5, \mathrm{~J}_{4-5} 2.0,4-\right.$ $\mathrm{H}), 4.36\left(1 \mathrm{H}, \mathrm{dd}, \mathrm{J}_{2-1} 5.0, \mathrm{~J}_{2-3} 2.5,2-\mathrm{H}\right), 4.44\left(2 \mathrm{H}, \mathrm{d}, \mathrm{J}_{6 \mathrm{a}, \mathrm{b}-5} 6.0,6 \mathrm{a}, \mathrm{b}-\mathrm{H}\right), 4.59(2 \mathrm{H}, \mathrm{qd}$, $\left.\mathrm{J}_{\mathrm{H}-\mathrm{F}} 7.0, \mathrm{~J}_{\mathrm{H}-\mathrm{H}} 1.0, \mathrm{CH}_{2} \mathrm{CF}_{3}\right), 4.65\left(1 \mathrm{H}, \mathrm{dd}, \mathrm{J}_{3-4} 7.5, \mathrm{~J}_{3-2} 2.5,3-\mathrm{H}\right), 5.53\left(1 \mathrm{H}, \mathrm{d}, \mathrm{J}_{1-2} 5.0,1-\right.$ $\mathrm{H}) ; \delta_{\mathrm{C}}\left(62 \mathrm{MHz} ; \mathrm{CDCl}_{3}\right) 24.2\left(\mathrm{CH}_{3}\right), 24.6\left(\mathrm{CH}_{3}\right), 25.6\left(\mathrm{CH}_{3}\right), 25.7\left(\mathrm{CH}_{3}\right), 66.6\left(\mathrm{q},{ }^{2} \mathrm{~J}_{\mathrm{C}-\mathrm{F}}\right.$ 39.4, $\left.\mathrm{CH}_{2} \mathrm{CF}_{3}\right), 70.0(\mathrm{CH}), 70.3(\mathrm{CH}), 70.4(\mathrm{CH}), 72.4(6-\mathrm{C}), 95.9(1-\mathrm{C}), 109.0\left(\mathrm{CH}_{3}-\right.$ 
$\left.C-\mathrm{CH}_{3}\right), 109.9\left(\mathrm{CH}_{3}-C-\mathrm{CH}_{3}\right), 121.5\left(\mathrm{q}, \mathrm{J}_{\mathrm{C}-\mathrm{F}} 277.5, \mathrm{CF}_{3}\right) ; \delta_{\mathrm{F}}\left(250 \mathrm{MHz} ; \mathrm{CDCl}_{3}\right)-74.09$; $\mathrm{m} / \mathrm{z}(\mathrm{FAB}) 423\left(\mathrm{MH}^{+}\right), \mathrm{C}_{14} \mathrm{H}_{22} \mathrm{~F}_{3} \mathrm{O}_{9} \mathrm{~S}$ requires 423.09367, Found 423.09351 (dev. $0.35)$.

1,3,4,6-Tetra-O-acetyl- $\alpha-D$-glucopyranose ${ }^{126}(31)$.

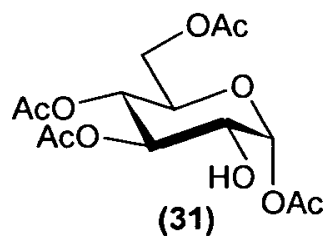

A portion of glucose monohydrate $(0.20 \mathrm{~g}, 1 \mathrm{mmol})$ followed by perchloric acid $(70 \%$ aqueous soln.) (4 drops) were added to acetic anhydride $\left(50 \mathrm{~cm}^{3}\right)$. The remaining glucose monohydrate $(13.00 \mathrm{~g}, 66 \mathrm{mmol})$ was added with cooling such that the temperature of $40-45^{\circ} \mathrm{C}$ was maintained. The solution was cooled to room temperature. Phosphorous tribromide $(24.77 \mathrm{~g}, 92 \mathrm{mmol})$ was added such that the temperature did not rise above $30^{\circ} \mathrm{C}$. After $45 \mathrm{~min}$ the reaction was cooled to $10^{\circ} \mathrm{C}$, sodium acetate soln. ( $40 \mathrm{~g}$ in $50 \mathrm{~cm}^{3}$ of water) was added such that a temperature of $40^{\circ} \mathrm{C}$ was maintained. After cooling to room temperature dichloromethane $\left(20 \mathrm{~cm}^{3}\right)$ was added and the mixture poured into a separating funnel $2 / 3$ full of ice. The product was extracted with dichloromethane $\left(3 \times 10 \mathrm{~cm}^{3}\right)$ the combined organic layers were washed with ice water $\left(50 \mathrm{~cm}^{3}\right)$, ice cold potassium hydrogen carbonate soln. $\left(50 \mathrm{~cm}^{3}\right)$ and ice water $\left(20 \mathrm{~cm}^{3}\right)$. The organic layer was then dried $\mathrm{MgSO}_{4}$ and the solvent evaporated under reduced pressure to give a yellow oil. Trituration with hot diethyl ether gave (31) $(3.37 \mathrm{~g}, 13 \%)$ as a colourless solid; $\mathrm{mp} 97-98^{\circ} \mathrm{C}$ (from ether) (lit., 126 98-100); $v_{\text {max }} / \mathrm{cm}^{-1} 3479,2916,1747,1971,1224,1015 ; \delta_{\mathrm{H}}\left(250 \mathrm{MHz} ; \mathrm{CDCl}_{3}\right.$ ) $1.96(3 \mathrm{H}, \mathrm{s}, \mathrm{OAc}), 1.99$ (6H, s, 2x OAc), $2.10(3 \mathrm{H}, \mathrm{s}, \mathrm{OAc}), 3.30(1 \mathrm{H}, \mathrm{bs}, \mathrm{OH}) 3.82$ $\left(1 \mathrm{H}, \mathrm{dd}, \mathrm{J}_{2-3} 9.5, \mathrm{~J}_{2-1} 3.5,2-\mathrm{H}\right), 3.93-3.99(2 \mathrm{H}, \mathrm{m}, 5,6 \mathrm{~b}-\mathrm{H}), 4.19(1 \mathrm{H}, \mathrm{dd}, \mathrm{J} 13.0$, J 4.5, $6 \mathrm{a}-\mathrm{H}), 4.99\left(1 \mathrm{H}, \mathrm{dd}, \mathrm{J}_{4-3} 9.5, \mathrm{~J} 9.5,4-\mathrm{H}\right), 5.19\left(1 \mathrm{H}, \mathrm{dd}, \mathrm{J}_{3-2} 9.5, \mathrm{~J}_{3-4} 9.5,3-\mathrm{H}\right), 6.14$ $\left(1 \mathrm{H}, \mathrm{d}, \mathrm{J}_{1-2} 3.5,1-\mathrm{H}\right) ; \delta_{\mathrm{C}}\left(62 \mathrm{MHz} ; \mathrm{CDCl}_{3}\right) 20.2\left(\mathrm{CH}_{3}\right), 20.3\left(\mathrm{CH}_{3}\right), 20.4\left(\mathrm{CH}_{3}\right), 20.5$ $\left(\mathrm{CH}_{3}\right), 61.3(6-\mathrm{C}), 67.3(\mathrm{CH}), 69.1(\mathrm{CH}), 69.2(\mathrm{CH}), 72.6(\mathrm{CH}), 91.0(1-\mathrm{C}), 169.1$ $(\mathrm{C}=\mathrm{O}), 169.3(\mathrm{C}=\mathrm{O}), 170.5(\mathrm{C}=\mathrm{O}), 170.9(\mathrm{C}=\mathrm{O}) ; \mathrm{m} / \mathrm{z}$ (Electrospray) $366\left(\mathrm{MNH}_{4}{ }^{+}\right)$. 
1,3,4,6-Tetra- $O$-acetyl- $\alpha$-D-glucopyranose 2-(2',2',2'-trifluoroethyl sulfate) (32).

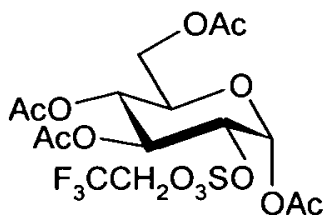

(32)

Sulfur trioxide/ pyridine complex $(473.0 \mathrm{mg}, 3.0 \mathrm{mmol})$ was added to a solution of (31) $(209.0 \mathrm{mg}, 0.60 \mathrm{mmol})$ in acetonitrile $\left(10 \mathrm{~cm}^{3}\right)$. The mixture was heated at $80^{\circ} \mathrm{C}$ for $1 \mathrm{~h}$, then cooled to room temperature. Diazo solution (24) $\left(20 \mathrm{~cm}^{3}\right)$ and citric acid monohydrate $(1 \mathrm{~g})$ were added and the reaction stirred for a further $16 \mathrm{~h}$. The solvent was evaporated under reduced pressure and the residue dissolved in water $\left(20 \mathrm{~cm}^{3}\right)$. The solution was extracted with diethyl ether $\left(3 \times 50 \mathrm{~cm}^{3}\right)$, the organic layers were combined, dried with $\mathrm{MgSO}_{4}$ and the solvent evaporated under reduced pressure to give a yellow oil. The product was purified by column chromatography (diethyl ether) to give (32) $(175.0 \mathrm{mg}, 60 \%)$ as a colourless gum; $[\alpha]_{\mathrm{D}}{ }^{16}+4.4^{\circ}(c 0.084$, $\mathrm{CHCl}_{3}$ ); $v_{\text {max }} / \mathrm{cm}^{-1} 1240,1750,2930 ; \delta_{\mathrm{H}}\left(250 \mathrm{MHz} ; \mathrm{CDCl}_{3}\right) 2.03$ (OAc), 2.06 (OAc), 2.07 (OAc), 2.20 (OAc), 4.02-4.14 (2H, m, 5, 6a-H), 4.27 (1H, dd, J 12.8, J 4.5, 6b$\mathrm{H}), 4.47\left(1 \mathrm{H}, \mathrm{dq}, \mathrm{J}_{\mathrm{H}-\mathrm{H}} 11.0, \mathrm{H}_{\mathrm{H}-\mathrm{F}} 7.5, \mathrm{CH}_{2} \mathrm{CF}_{3}\right), 4.57\left(1 \mathrm{H}, \mathrm{dq}, \mathrm{J}_{\mathrm{H}-\mathrm{H}} 11.0, \mathrm{H}_{\mathrm{H}-\mathrm{F}} 7.5\right.$, $\left.\mathrm{CH}_{2} \mathrm{CF}_{3}\right), 4.82\left(1 \mathrm{H}, \mathrm{dd}, \mathrm{J}_{2-3} 10.0, \mathrm{~J}_{2-1} 3.5,2-\mathrm{H}\right), 5.14\left(1 \mathrm{H}, \mathrm{dd}, \mathrm{J}_{3-2} 10.0, \mathrm{~J}_{3-4} 10.0,3-\mathrm{H}\right)$, $5.51\left(1 \mathrm{H}, \mathrm{dd}, \mathrm{J}_{4-3} 10.0, \mathrm{~J} 10.0,4-\mathrm{H}\right), 6.46\left(1 \mathrm{H}, \mathrm{d}, \mathrm{J}_{1-2} 3.5,1-\mathrm{H}\right) ; \delta_{\mathrm{C}}\left(62 \mathrm{MHz} ; \mathrm{CDCl}_{3}\right)$ 20.2 (2 x OAc), 20.3 (OAc), 10.4 (OAc), 60.8, 67.1 (q, ${ }^{2} \mathrm{~J}_{\mathrm{C}-\mathrm{F}} 38.7, \mathrm{CH}_{2} \mathrm{CF}_{3}$ ), 67.2, 69.0, 69.4, 78.1, 87.8, $121.1\left(\mathrm{q}, \mathrm{J}_{\mathrm{C}-\mathrm{F}} 277.4, C F_{3}\right), 168.2(\mathrm{C}=\mathrm{O}), 168.9(\mathrm{C}=\mathrm{O}), 169.7$ $(\mathrm{C}=\mathrm{O}), 170.2(\mathrm{C}=\mathrm{O}) ; \delta_{\mathrm{F}}\left(250 \mathrm{MHz} ; \mathrm{CDCl}_{3}\right)-74.23 ; \mathrm{m} / \mathrm{z}$ (Electrospray) $528\left(\mathrm{MNH}_{4}^{+}\right)$.

Methyl 2,3,6-tri- $O$-benzoyl- $\alpha$-D-glucopyranoside (34) and Methyl 2,3,4,6-tetraO-benzoyl- $\alpha$-D-glucopyranoside 128 (106).

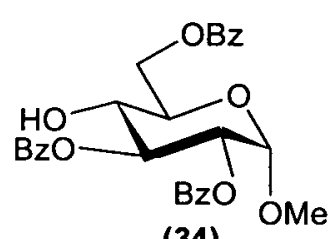

(34)

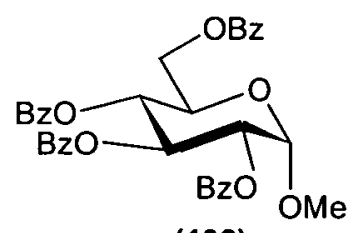

(106)

To a solution of methyl $\alpha$-D-glucopyranoside $(3.88 \mathrm{~g}, 20 \mathrm{mmol})$ in pyridine $\left(160 \mathrm{~cm}^{3}\right)$ at $-40^{\circ} \mathrm{C}$ was added benzoyl chloride $(8.71 \mathrm{~g}, 62 \mathrm{mmol})$. The reaction was stirred at 
room temperature for $16 \mathrm{hr}$. The pyridine was removed under reduced pressure and the resulting oil dissolved in dichloromethane $\left(75 \mathrm{~cm}^{3}\right)$, extracted with sat. potassium bicarbonate soln. $\left(50 \mathrm{~cm}^{3}\right)$ and water $\left(50 \mathrm{~cm}^{3}\right)$. The organic layer was then dried with $\mathrm{MgSO}_{4}$ and evaporated under reduced pressure to give a yellow oil. Column chromatography (10: 1 toluene : ethyl acetate) gave (106) $(2.13 \mathrm{~g}, 21 \%)\left(\mathrm{R}_{\mathrm{F}} 0.45\right)$; mp 105-107 (lit., 128 106-108); $v_{\max } / \mathrm{cm}^{-1} 2916,1725,1269,1098,1028,711 ; \delta_{\mathrm{H}}$ $\left(250 \mathrm{MHz} ; \mathrm{CDCl}_{3}\right) 3.48\left(3 \mathrm{H}, \mathrm{s}, \mathrm{OCH}_{3}\right), 4.38-4.56(2 \mathrm{H}, \mathrm{m}, 6 \mathrm{~b}-\mathrm{H}, 5-\mathrm{H}), 4.61(1 \mathrm{H}, \mathrm{dd}, \mathrm{J}$ 12.0, J 2.5, 6a-H), $5.25\left(1 \mathrm{H}, \mathrm{d}_{, \mathrm{J}_{1-2}} 3.5,1-\mathrm{H}\right), 5.30\left(1 \mathrm{H}, \mathrm{dd}, \mathrm{J}_{2-3} 10.0, \mathrm{~J}_{2-1} 3.5,2-\mathrm{H}\right)$, $5.69\left(1 \mathrm{H}, \mathrm{dd}, \mathrm{J}_{4-3} 10.0, \mathrm{~J}_{4-5} 10.0,4-\mathrm{H}\right), 6.19\left(1 \mathrm{H}, \mathrm{dd}, \mathrm{J}_{3-4} 10.0, \mathrm{~J}_{3-2} 10.0,2-\mathrm{H}\right), 7.27-$ $7.58(12 \mathrm{H}, \mathrm{m}, \mathrm{Ar}), 7.85-8.07(8 \mathrm{H}, \mathrm{m}, \mathrm{Ar}) ; \delta_{\mathrm{C}}\left(62 \mathrm{MHz} ; \mathrm{CDCl}_{3}\right) 55.4\left(\mathrm{OCH}_{3}\right), 62.8(6-$ C), $67.5(\mathrm{CH}), 69.4(\mathrm{CH}), 70.2(\mathrm{CH}), 71.8(\mathrm{CH}), 96.8(1-\mathrm{C}), 128.1$ (2 x C-Arom.), 128.2 (6 x C-Arom.), 128.6 ( 1 x C-Arom), 128.8 (1 x C-Arom), 128.9 ( 1 x C-Arom), 129.3 (1 x C-Arom), 129.4 (2 x C-Arom.), 129.5 (2 x C-Arom.), 129.6 ( 2 x C-Arom), 129.7 ( $2 \times$ C-Arom), 132.9 ( $2 \times$ C-Arom), 133.2 ( $2 \times$ C-Arom), 165.1 (C=O), 165.6 $(\mathrm{C}=\mathrm{O}), 165.6(\mathrm{C}=\mathrm{O}), 165.9(\mathrm{C}=\mathrm{O}) ; \mathrm{m} / \mathrm{z}$ (Electrospray) $633\left(\mathrm{MNa}^{+}\right)$. and (34) $(2.01 \mathrm{~g}$, 20\%) ( $\mathrm{R}_{\mathrm{F}}$ 0.2); mp 124-127 (lit., 128 125-126); $v_{\max } / \mathrm{cm}^{-1} 3483,2916,1721,1463$, $1273,1107,712 ; \delta_{\mathrm{H}}\left(250 \mathrm{MHz} ; \mathrm{CDCl}_{3}\right) 3.36\left(1 \mathrm{H}, \mathrm{d}, \mathrm{J}_{\mathrm{OH}-3} 4.5, \mathrm{OH}\right), 3.45(3 \mathrm{H}, \mathrm{s}$, OMe), $3.87\left(1 \mathrm{H}\right.$, ddd, $\left.\mathrm{J}_{3-2} 10.0, \mathrm{~J}_{3-4} 10.0, \mathrm{~J}_{3-\mathrm{OH}} 4.5,3-\mathrm{H}\right), 4.09(1 \mathrm{H}, \mathrm{m} ., 5-\mathrm{H}), 4.61(1 \mathrm{H}$, $\left.\mathrm{dd}, \mathrm{J}_{6 \mathrm{a}-6 \mathrm{~b}} 12.0, \mathrm{~J} 2.0,6 \mathrm{a}-\mathrm{H}\right), 4.80\left(1 \mathrm{H}, \mathrm{dd}, \mathrm{J}_{6 \mathrm{~b}-6 \mathrm{a}} 12.0, \mathrm{~J} 4.5,6 \mathrm{~b}-\mathrm{H}\right), 5.12\left(1 \mathrm{H}, \mathrm{d}, \mathrm{J}_{1-2} 3.5\right.$, 1-H), $5.26\left(1 \mathrm{H}, \mathrm{dd}, \mathrm{J}_{2-3} 10.0, \mathrm{~J}_{2-1} 3.5,2-\mathrm{H}\right), 5.78\left(1 \mathrm{H}, \mathrm{dd}, \mathrm{J}_{4-3} 10.0, \mathrm{~J} 10.0,4-\mathrm{H}\right), 7.26-$ $7.63\left(9 \mathrm{H}, \mathrm{m}\right.$, Arom.), 7.96-8.14 (6H, m, Arom.); $\delta_{\mathrm{C}}\left(62 \mathrm{MHz} ; \mathrm{CDCl}_{3}\right) 55.3\left(\mathrm{OCH}_{3}\right)$, $63.3(6-\mathrm{C}), 69.5(\mathrm{CH}), 69.9(\mathrm{CH}), 71.2(\mathrm{CH}), 73.7(\mathrm{CH}), 96.9(1-\mathrm{C}), 128.2(3 \times \mathrm{C}-$ Arom.), 128.3 (3 x C-Arom.), 128.9 (1 x C-Arom), 129.0 (1 x C-Arom), 129.5 (1 x C-Arom), 129.6 (3 x C-Arom.), 129.7 (3 x C-Arom.), 133.1 (1 x C-Arom), 133.2 (2 x C-Arom), $165.8(\mathrm{C}=\mathrm{O}), 166.8(\mathrm{C}=\mathrm{O}), 167.1(\mathrm{C}=\mathrm{O}) ; \mathrm{m} / \mathrm{z}$ (Electrospray) $529\left(\mathrm{MNa}^{+}\right)$. 

sulfate) (35).

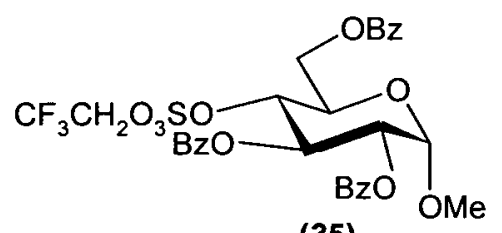

(35)

Sulfur trioxide/ pyridine complex (954mg, 6.0mmol) was added to a solution of (34) $(304 \mathrm{mg}, 0.60 \mathrm{mmol})$ in acetonitrile $\left(10 \mathrm{~cm}^{3}\right)$. The mixture was heated at $80^{\circ} \mathrm{C}$ for $0.5 \mathrm{~h}$, then cooled to room temperature. Diazo solution (24) $\left(20 \mathrm{~cm}^{3}\right)$ and citric acid monohydrate $(1 \mathrm{~g})$ were added and the reaction stirred for a further $16 \mathrm{~h}$. The solvent was evaporated under reduced pressure and the residue dissolved in water $\left(20 \mathrm{~cm}^{3}\right)$. The solution was extracted with diethyl ether $\left(3 \times 50 \mathrm{~cm}^{3}\right)$, the organic layers were combined, dried with $\mathrm{MgSO}_{4}$ and the solvent evaporated under reduced pressure to give a yellow oil. The product was purified by column chromatography $(1: 1$ dichloromethane : hexane) to give (35) $(320 \mathrm{mg}, 80 \%)$ as a colourless solid; $\mathrm{mp} 56-$ $58^{\circ} \mathrm{C} ;[\alpha]_{\mathrm{D}}^{16}+117.8\left(\mathrm{c} 0.0225, \mathrm{CHCl}_{3}\right.$ ); (Found: C, 54.05; H, 4.2. $\mathrm{C}_{30} \mathrm{H}_{27} \mathrm{~F}_{3} \mathrm{O}_{12} \mathrm{~S}$ requires $\mathrm{C}, 53.9 ; \mathrm{H}, 4.1 \%) ; v_{\max } / \mathrm{cm}^{-1} 1693,1240,670 ; \delta_{\mathrm{H}}\left(250 \mathrm{MHz} ; \mathrm{CDCl}_{3}\right) 3.44$ $\left(3 \mathrm{H}, \mathrm{s}, \mathrm{OCH}_{3}\right), 4.26-4.49\left(3 \mathrm{H}, \mathrm{m}, 5-\mathrm{H}, \mathrm{CH}_{3} \mathrm{CF}_{3}\right), 4.53\left(1 \mathrm{H}, \mathrm{dd}, \mathrm{J}_{6 \mathrm{a}-6 \mathrm{~b}} 12.5, \mathrm{~J} 4.5,6 \mathrm{a}-\right.$ $\mathrm{H}), 4.83\left(1 \mathrm{H}, \mathrm{dd}, \mathrm{J}_{6 \mathrm{~b}-6 \mathrm{a}} 12.5, \mathrm{~J} 4.5,6 \mathrm{~b}-\mathrm{H}\right), 5.16\left(1 \mathrm{H}, \mathrm{dd}, \mathrm{J}_{4-3} 9.5, \mathrm{~J} 9.5,4-\mathrm{H}\right), 5.17(1 \mathrm{H}$, $\left.\mathrm{d}, \mathrm{J}_{1-2} 3.5,1-\mathrm{H}\right), 5.19\left(1 \mathrm{H}, \mathrm{dd}, \mathrm{J}_{2-3} 9.5, \mathrm{~J}_{2-1} 3.5,2-\mathrm{H}\right), 6.13\left(1 \mathrm{H}, \mathrm{dd}, \mathrm{J}_{3-2} 9.5, \mathrm{~J}_{3-4} 9.5,3-\right.$ $\mathrm{H})$, 7.34-7.64 (9H, m, Ar), 7.93-8.14 (6H, m, Ar); $\delta_{\mathrm{C}}\left(62 \mathrm{MHz} ; \mathrm{CDCl}_{3}\right) 55.7\left(\mathrm{OCH}_{3}\right)$, $61.7(6-\mathrm{C}), 66.8(\mathrm{CH}), 67.2\left(\mathrm{q},{ }^{2} \mathrm{~J}_{\mathrm{C}-\mathrm{F}} 38.9, \mathrm{CH}_{2} \mathrm{CF}_{3}\right), 69.4(\mathrm{CH}), 71.5(\mathrm{CH}), 79.6(\mathrm{CH})$, $96.6(\mathrm{CH}), 121.4\left(\mathrm{q},{ }^{1} \mathrm{~J}_{\mathrm{C}-\mathrm{F}} 277.4, \mathrm{CF}_{3}\right), 128.3$ (4x Ar), 128.4 (3x Ar), 128.4 (2x Ar), 129.6 (2x Ar), 129.7 (2x Ar), 129.8 (2x Ar), 133.2 (1x Ar), $133.4(2 \mathrm{x} \mathrm{Ar}) ; \delta_{\mathrm{F}}$ (250MHz; $\left.\mathrm{CDCl}_{3}\right)-74.45 ; \mathrm{m} / \mathrm{z}(\mathrm{FAB}) 669\left(\mathrm{MH}^{+}\right), \mathrm{C}_{30} \mathrm{H}_{28} \mathrm{~F}_{3} \mathrm{O}_{12} \mathrm{~S}$ requires 669.12536, Found 669.12482 (dev. -0.81). 


\section{2,3,4,6-Tetra- $O$-benzyl-1-sulfo-D-glucopyranose, pyridinium salt (37).}

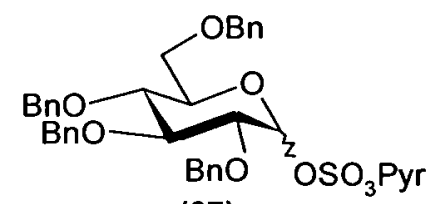

(37)

Sulfur trioxide/ pyridine complex $(34 \mathrm{mg}, 0.22 \mathrm{mmol})$ was added to a solution of 2,3,4,6-tetra- $O$-benzyl- $\alpha$-D-glucopyranose $(100.0 \mathrm{mg}, 0.18 \mathrm{mmol})$ in acetonitrile $\left(2 \mathrm{~cm}^{3}\right)$. The mixture was heated at $80^{\circ} \mathrm{C}$ for $2 \mathrm{~h}$, then cooled to room temperature, the solution was used without further purification; m/z (-ve Electrospray) $619\left(\mathrm{M}^{-}\right)$.

\section{2,3,4,6-Tetra- $O$-benzyl-D-glucopyranosyl acetimidate (38).}

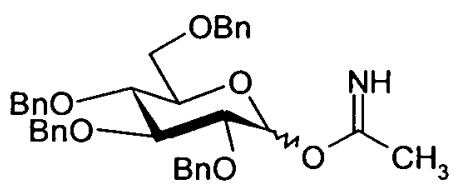

(38)

To a solution of (37) $\left(2 \mathrm{~cm}^{3}\right)$ was added a solution of (24) $\left(10 \mathrm{~cm}^{3}\right)$ and citric acid (1g). After $16 \mathrm{~h}$ two products were observed by TLC (9: 1; dichloromethane : diethyl ether). The solvent was evaporated under reduced pressure and the products purified by column chromatography (9: 1 ; dichloromethane : diethyl ether) to give $2,3,4,6-$ tetra- $O$-benzyl-D-glucopyranose $(20 \mathrm{mg}, 20 \%$ of original starting material); $\mathrm{m} / \mathrm{z}$ (Electrospray) $558\left(\mathrm{MNH}_{4}^{+}\right)$and (38) (30mg); m/z (Electrospray) $582\left(\mathrm{MH}^{+}\right)$.

Methyl 3-sulfo- $\beta$-D-galactopyranoside, trimethylamine salt ${ }^{92}$ (39).

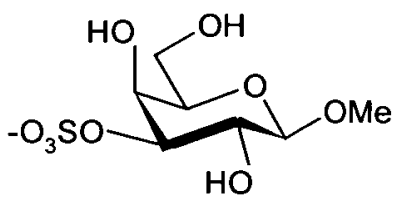

(39)

A solution of methyl $\beta$-D-galactopyranoside $(200 \mathrm{mg}, 1.0 \mathrm{mmol})$ and dibutyltin oxide $(256 \mathrm{mg}, 1.5 \mathrm{mmol})$ in methanol $\left(10 \mathrm{~cm}^{3}\right)$ was heated at reflux for $1.5 \mathrm{~h}$ under nitrogen. The solvent was removed and replaced with THF $\left(10 \mathrm{~cm}^{3}\right)$, sulfur trioxide trimethylamine complex $(286 \mathrm{mg}, 2.0 \mathrm{mmol})$ was added and the reaction stirred for a further $24 \mathrm{~h}$. The solvent was evaporated under reduced pressure to give (39) as a 
colourless solid. The compound was used in subsequent reactions without further purification.

Methyl $\beta$-D-galactopyranoside 3-(2',2',2'-trifluoroethyl sulfate) (41).

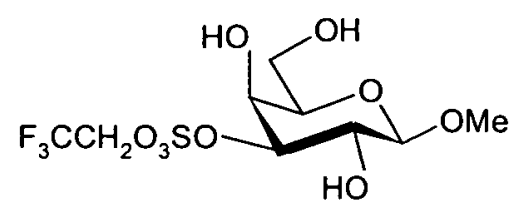

(41)

The crude sulfate (39) was suspended in acetonitrile. 2,2,2-trifluorodiazoethane soln $\left(20 \mathrm{~cm}^{3}\right)$ and citric acid monohydrate $(1.5 \mathrm{~g})$ were added and the reaction stirred for 16h. The solvent was evaporated under reduced pressure and the resulting solid purified by column chromatography (1:1 diethyl ether : ethyl acetate) to give (41) $(163 \mathrm{mg}, 46 \%)$ as a colourless oil; $[\alpha]_{\mathrm{D}}{ }^{16}-20.9$ (c 0.033 in $\left.\mathrm{MeOH}\right) ; v_{\max } / \mathrm{cm}^{-1} 3297$, 2929,$1420 ; \delta_{\mathrm{H}}\left(250 \mathrm{MHz} ; \mathrm{CD}_{3} \mathrm{OD}\right) 3.62-3.69(3 \mathrm{H}, \mathrm{m}, 6 \mathrm{a}, 6 \mathrm{~b}, 5-\mathrm{H}) 3.81(\mathrm{OMe}), 3.82$ $\left(1 \mathrm{H}, \mathrm{dd}, \mathrm{J}_{2-3} 10.0, \mathrm{~J}_{2-1} 7.5,2-\mathrm{H}\right), 4.31\left(1 \mathrm{H}, \mathrm{dd}, \mathrm{J}_{4-3} 3.5, \mathrm{~J} 1.0,4-\mathrm{H}\right), 4.32\left(1 \mathrm{H}, \mathrm{d}, \mathrm{J}_{1-2}\right.$ $10.0,1-\mathrm{H}), 4.64\left(1 \mathrm{H}, \mathrm{dd}, \mathrm{J}_{3-2} 10.0, \mathrm{~J}_{3-4} 3.5,3-\mathrm{H}\right), 4.87\left(1 \mathrm{H}, \mathrm{dq}, \mathrm{J}_{\mathrm{Ha}-\mathrm{Hb}} 12.0, \mathrm{~J}_{\mathrm{H}-\mathrm{F}} 8.0\right.$, $\left.\mathrm{CH}_{2} \mathrm{CF}_{3}\right), 5.01\left(1 \mathrm{H}, \mathrm{dq}, \mathrm{J}_{\mathrm{Ha}-\mathrm{Hb}} 12.0, \mathrm{~J}_{\mathrm{H}-\mathrm{F}} 8.0, \mathrm{CH}_{2} \mathrm{CF}_{3}\right) ; \delta_{\mathrm{C}}\left(62 \mathrm{MHz} ; \mathrm{CD}_{3} \mathrm{OD}\right) 55.5$, $59.9,66.0\left(\mathrm{q},{ }^{2} \mathrm{~J}_{\mathrm{CF}} 37.9, \mathrm{CH}_{2} \mathrm{CF}_{3}\right), 66.3,67.8,73.7,86.6,103.7,121.5\left(\mathrm{q}, \mathrm{J}_{\mathrm{C}-\mathrm{F}} 276.5\right.$, $\left.\mathrm{CF}_{3}\right), \delta_{\mathrm{F}}\left(250 \mathrm{MHz} ; \mathrm{CD}_{3} \mathrm{OD}\right)-78.30$.

Methyl 4;6-O-benzylidene-2-sulfo- $\alpha$-D-glucopyranoside, pyridinium salt ${ }^{93}$ (40).

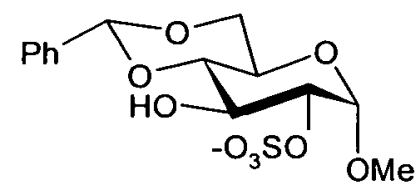

(40)

A solution of methyl 4;6-O-benzylidene- $\alpha$-D-glucopyranoside (400 $\mathrm{mg}, 1.42 \mathrm{mmol}$ ) and dibutyltin oxide $(352 \mathrm{mg}, 1.42 \mathrm{mmol})$ in toluene was refluxed under Dean-Stark conditions and nitrogen for $20 \mathrm{hrs}$. The toluene $\left(100 \mathrm{~cm}^{3}\right)$ was evaporated under reduced pressure and replaced with DMF $\left(5 \mathrm{~cm}^{3}\right)$. A solution of sulfur trioxide trimethylamine complex in DMF $\left(3 \mathrm{~cm}^{3}\right)$ was added and the reaction stirred for a further $2 \mathrm{~h}$. The solvent was evaporated under reduced pressure to give crude (40). 
Methyl 4;6-O-benzylidene- $\alpha$-D-glucopyranoside 2-(2',2',2'-trifluoroethyl sulfate) (43).

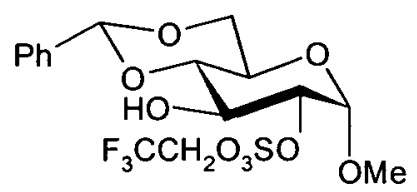

(43)

The crude sulfate (40) was suspended in acetonitrile $\left(20 \mathrm{~cm}^{3}\right) .(24)$ soln. $\left(20 \mathrm{~cm}^{3}\right)$ and citric acid (1g) were added and the mixture was stirred for $16 \mathrm{~h}$. The solvent was then evaporated under reduced pressure and the resulting solid purified by column chromatography (dichloromethane) to give (43) $(477 \mathrm{mg}, 75 \%)$ as a colourless solid; $[\alpha]_{\mathrm{D}}{ }^{16}+96.1$ (c 0.0165 in $\mathrm{CHCl}_{3}$ ); (Found: $\mathrm{C}, 43.8 ; \mathrm{H}, 4.5 . \mathrm{C}_{16} \mathrm{H}_{19} \mathrm{~F}_{3} \mathrm{O}_{9} \mathrm{~S}$ requires ,C, $43.3 ; \mathrm{H}, 4.3 \%) ; v_{\max } / \mathrm{cm}^{-1} 3495,2959,1243 ; \delta_{\mathrm{H}}\left(250 \mathrm{MHz} ; \mathrm{CDCl}_{3}\right) 2.75\left(1 \mathrm{H}, \mathrm{d}, \mathrm{J}_{\mathrm{OH}-3}\right.$ 2.5, OH-(C3)), $3.46\left(3 \mathrm{H}, \mathrm{s}, \mathrm{OCH}_{3}\right), 3.50\left(1 \mathrm{H}, \mathrm{dd}, \mathrm{J}_{6 \mathrm{~b}-6 \mathrm{a}} 10.0, \mathrm{~J}_{6 \mathrm{~b}-5} 10.0,6 \mathrm{~b}-\mathrm{H}\right), 3.73$ $\left(1 \mathrm{H}, \mathrm{dd}, \mathrm{J}_{6 \mathrm{a}-6 \mathrm{~b}} 10.0, \mathrm{~J}_{6 \mathrm{a}-5} 10.0,6 \mathrm{a}-\mathrm{H}\right), 3.84\left(1 \mathrm{H}, \mathrm{ddd}, \mathrm{J}_{5-6 \mathrm{a}} 10.0, \mathrm{~J}_{5-6 \mathrm{~b}} 10.0, \mathrm{~J}_{5-4} 4.5,5-\mathrm{H}\right)$, $4.20\left(1 \mathrm{H}, \mathrm{ddd}, \mathrm{J}_{3-4} 9.5, \mathrm{~J}_{3-2} 9.5, \mathrm{~J}_{3-\mathrm{OH}} 2.5,3-\mathrm{H}\right), 4.30\left(1 \mathrm{H}, \mathrm{dd}, \mathrm{J}_{4-3} 9.5, \mathrm{~J}_{4-5} 4.5,4-\mathrm{H}\right)$, $4.49\left(1 \mathrm{H}, \mathrm{dd}, \mathrm{J}_{2-3} 9.5, \mathrm{~J}_{2-1} 3.5,2-\mathrm{H}\right), 4.54\left(1 \mathrm{H}, \mathrm{dq}, \mathrm{J}_{\mathrm{Ha}-\mathrm{Hb}} 11.5, \mathrm{~J}_{\mathrm{H}-\mathrm{F}} 8.0, \mathrm{CH}_{2} \mathrm{CF}_{3}\right) 4.72$ $\left(1 \mathrm{H}, \mathrm{dq}, \mathrm{J}_{\mathrm{Ha}-\mathrm{Hb}} 11.5, \mathrm{~J}_{\mathrm{H}-\mathrm{F}} 8.0, \mathrm{CH}_{2} \mathrm{CF}_{3}\right), 5.05\left(1 \mathrm{H}, \mathrm{d}, \mathrm{J}_{1-2} 3.5,1-\mathrm{H}\right), 5.53(1 \mathrm{H}, \mathrm{s}, \mathrm{CH}-$ $\mathrm{Ph})$, 7.35-7.41 (3H, m, Arom.) 7.44-7.49 (2H, m, Arom.); $\delta_{\mathrm{C}}\left(62 \mathrm{MHz}, \mathrm{CDCl}_{3}\right) 55.6$ $\left(\mathrm{OCH}_{3}\right), 61.6,67.0\left(\mathrm{q},{ }^{2} \mathrm{~J}_{\mathrm{C}-\mathrm{F}} 38.5, \mathrm{CH}_{2} \mathrm{CF}_{3}\right), 68.1,68.5(6-\mathrm{C}), 81.0,82.0,121.5\left(\mathrm{q}, \mathrm{J}_{\mathrm{C}-\mathrm{F}}\right.$ 277.4, $\mathrm{CF}_{3}$ ), 126.1 (2x CH Arom) 128.3 (2 x CH Arom), 129.4 (CH Arom), 136.5 (Arom); $\delta_{\mathrm{F}}\left(250 \mathrm{MHz} ; \mathrm{CDCl}_{3}\right)-74.02 ; \mathrm{m} / \mathrm{z}(\mathrm{FAB}) 445\left(\mathrm{MH}^{+}\right), \mathrm{C}_{16} \mathrm{H}_{20} \mathrm{~F}_{3} \mathrm{O}_{9} \mathrm{~S}$ requires 445.07802 found 445.07860 (dev. 1.31).

\section{Phenyl 1-deoxy-4-O-( $\beta$-D-galactopyranosyl 3'-(2',,2',2''-trifluoroethyl sulfate))-} 1-thio- $\beta$-glucopyranoside (46).

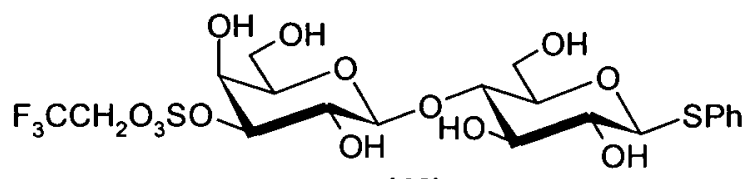

(46)

To a solution of phenyl 1-deoxy-4-O-(3'- $O$-sulfo- $\beta$-D-galactopyranosyl)-1-thio- $\beta$ glucopyranoside 92 (50mg, 93 $\mu \mathrm{mol})$ was added (24) soln. $\left(10 \mathrm{~cm}^{3}\right)$ and citric acid 
monohydrate. The reaction was stirred for $20 \mathrm{~h}$, the solvent was then evaporated under reduced pressure and the resulting oil was dissolved in ethyl acetate $\left(25 \mathrm{~cm}^{3}\right)$ and extracted with sat. potassium bicarbonate soln. $\left(20 \mathrm{~cm}^{3}\right)$ and brine $\left(20 \mathrm{~cm}^{3}\right)$. The organic layer was then dried with $\mathrm{MgSO}_{4}$ and the solvent evaporated under reduced pressure. The product was purified by column chromatography (ethyl acetate) to give (46) $(37 \mathrm{mg}, 53 \%)$ as a colourless solid; $\mathrm{mp} 64-68^{\circ} \mathrm{C}$ (from ethyl acetate); $[\alpha]_{\mathrm{D}}{ }^{16}-3.8$ (c 0.0045 in $\mathrm{MeOH}) ; v_{\max } / \mathrm{cm}^{-1} 3402,2920,2880,1584,1240 ; \delta_{\mathrm{H}}\left(250 \mathrm{MHz} ; \mathrm{CD}_{3} \mathrm{OD}\right)$ $3.36\left(1 \mathrm{H}, \mathrm{dd}, \mathrm{J}_{3-4} 9.8, \mathrm{~J}_{3-2} 8.5,3-\mathrm{H}\right), 3.38-3.49(1 \mathrm{H}, \mathrm{m}, 5-\mathrm{H}), 3.63\left(1 \mathrm{H}, \mathrm{dd}, \mathrm{J}_{2-1} 8.5, \mathrm{~J}_{2-3}\right.$ 8.5, 2-H), $3.68\left(1 \mathrm{H}, \mathrm{dd}, \mathrm{J}_{4-3} 10.0, \mathrm{~J} 9.5,4-\mathrm{H}\right), 3.72-3.78\left(1 \mathrm{H}, \mathrm{m}, 5^{\prime}-\mathrm{H}\right), 3.78-3.91(5 \mathrm{H}$, m, 2',6a, 6b, 6a', 6b'-H), 3.91-4.04 (1H, m, 4'-H), 4.28 (1H, d, J 3.0, 1'-H), 4.61 $\left(1 \mathrm{H}, \mathrm{d}, \mathrm{J}_{1-2} 8.0,1-\mathrm{H}\right), 4.60-4.73\left(2 \mathrm{H}, \mathrm{m}, 3^{\prime}-\mathrm{H}, \mathrm{CH}_{2} \mathrm{CF}_{3}\right), 4.81-4.95\left(1 \mathrm{H}, \mathrm{m}, \mathrm{CH}_{2} \mathrm{CF}_{3}\right)$, 7.10-7.45 (3H, m, Arom.), 7.63-7.68 (2H, m, Arom.); $\delta_{\mathrm{C}}\left(62 \mathrm{MHz} ; \mathrm{CD}_{3} \mathrm{OD}\right) 59.9$, $60.1\left(2 \times \mathrm{CH}_{2}\right), 66.0(\mathrm{CH}), 66.3\left(\mathrm{q}, \mathrm{J}_{\mathrm{C}-\mathrm{F}} 37.9, \mathrm{CH}_{2} \mathrm{CF}_{3}\right), 67.9(\mathrm{CH}), 71.5(\mathrm{CH}), 74.2$ $(\mathrm{CH}), 76.0(\mathrm{CH}), 77.9(\mathrm{CH}), 78.6(\mathrm{CH}), 86.3(\mathrm{CH}), 87.2(\mathrm{CH}), 102.4(\mathrm{CH}), 126.1(\mathrm{q}$, $\left.\mathrm{J}_{\mathrm{C}-\mathrm{F}} 276.3, \mathrm{CF}_{3}\right), 126.5$ (CH, Arom.), 127.9 (2 x CH, Arom), 131.1 (2 x CH, Arom), 132.9 (C, Arom.); $\delta_{\mathrm{F}}\left(250 \mathrm{MHz} ; \mathrm{CD}_{3} \mathrm{OD}\right)-78.34 ; \mathrm{m} / \mathrm{z}$ (Electrospray) $619\left(\mathrm{MNa}^{+}\right)$, (FAB) $597.08975\left(\mathrm{MH}^{+} \mathrm{C}_{20} \mathrm{H}_{28} \mathrm{~F}_{3} \mathrm{O}_{13} \mathrm{~S}_{2}\right.$ requires 597.09235 (dev. -4.35)).

\section{D-Glucose 3-(2',2',2'-trifluoroethyl sulfate) (47).}

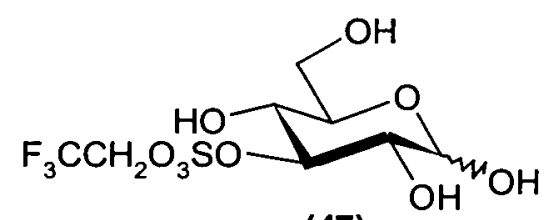

(47)

A mixture of (20) $(200 \mathrm{mg}, 0.5 \mathrm{mmol})$, trifluoroacetic acid $\left(8 \mathrm{~cm}^{3}\right)$ and ethanol $\left(2 \mathrm{~cm}^{3}\right)$ was stirred at room temperature for $1.5 \mathrm{~h}$. Potassium bicarbonate soln. (sat.) $\left(20 \mathrm{~cm}^{3}\right)$ was added and the mixture extracted with ethyl acetate $\left(3 \mathrm{x} 25 \mathrm{~cm}^{3}\right)$. The organic layers were dried with $\mathrm{MgSO}_{4}$ and the solvent evaporated under reduced pressure. The resulting oil was purified by column chromatography (ethyl acetate) to give (47) $\left(121 \mathrm{mg}, 75 \%\right.$ ) as a colourless gum (mixture of anomers $\alpha / \beta \sim 1: 3$ ); $v_{\max } / \mathrm{cm}^{-1} 3490$, 2930, 1240, 1080; $\delta_{\mathrm{H}}\left(250 \mathrm{MHz} ; \mathrm{CD}_{3} \mathrm{OD}\right) 3.69\left(1 \mathrm{H}, \mathrm{dd}, \mathrm{J}_{3-4} 9.5, \mathrm{~J}_{3-2} 9.5,3-\mathrm{H}\right), 3.70$ $\left(1 \mathrm{H}, \mathrm{dd}, \mathrm{J}_{2-3} 9.5, \mathrm{~J}_{2-1} 3.5,2-\mathrm{H}\right), 3.79\left(1 \mathrm{H}, \mathrm{dd}, \mathrm{J}_{4-3} 9.5, \mathrm{~J} 7.0,4-\mathrm{H}\right), 3.83-3.96(3 \mathrm{H}, \mathrm{m}, 5$, 
6a, 6b-H), $5.24\left(1 \mathrm{H}, \mathrm{d}, \mathrm{J}_{1-2} 3.5,1-\mathrm{H}\right) ; \delta_{\mathrm{C}}\left(62 \mathrm{MHz} ; \mathrm{CD}_{3} \mathrm{OD}\right)(\beta-$ form) $60.1(6-\mathrm{C}), 65.9$ $\left(\mathrm{q},{ }^{2} \mathrm{~J}_{\mathrm{C}-\mathrm{F}} 37.8, \mathrm{CH}_{2} \mathrm{CF}_{3}\right), 67.5,69.9,71.0,89.5,92.2,121.8\left(\mathrm{q}, \mathrm{J}_{\mathrm{C}-\mathrm{F}} 276.6, \mathrm{CF}_{3}\right) ;(\alpha-$ form) 60.3 (6-C), $65.9\left(\mathrm{q},{ }^{2} \mathrm{~J}_{\mathrm{C}-\mathrm{F}} 37.8, \mathrm{CH}_{2} \mathrm{CF}_{3}\right), 67.5,72.4,75.4,90.5,96.0,121.8(\mathrm{q}$, $\left.\mathrm{J}_{\mathrm{C}-\mathrm{F}} 276.6, \mathrm{CF}_{3}\right) ; \delta_{\mathrm{F}}\left(250 \mathrm{MHz} ; \mathrm{CD}_{3} \mathrm{OD}\right)(\beta$-form) -72.17; ( $\alpha$-form) -73.58; m/z (FAB) $381\left(\mathrm{MK}^{+}\right)$.

\section{D-Glucose 6-(2',2',2'-trifluoroethyl sulfate) (48).}

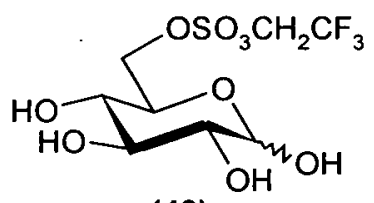

(48)

To a solution of (29) $(100 \mathrm{mg}, 0.2 \mathrm{mmol})$ in methanol $\left(20 \mathrm{~cm}^{3}\right)$ was added sodium methoxide $(50 \mathrm{mg}, 1.0 \mathrm{mmol})$. The mixture was stirred at room temperature for $2 \mathrm{~h}$. The solvent was then evaporated under reduced pressure and the product purified by column chromatography (7: 3; dichloromethane : methanol) to give (48) (44mg, $66 \%$ ) as a colourless solid; $[\alpha]_{\mathrm{D}}{ }^{16}-6.4(c 0.0025$ in $\mathrm{MeOH}$ ); (Found: $\mathrm{C}, 25.9 ; \mathrm{H}, 3.6$. $\mathrm{C}_{16} \mathrm{H}_{27} \mathrm{~F}_{6} \mathrm{KO}_{19} \mathrm{~S}_{2}$ requires $\left.\mathrm{C}, 25.95 ; \mathrm{H}, 3.7 \%\right) ; v_{\max } / \mathrm{cm}^{-1} 3490,2930,1240 ; \delta_{\mathrm{H}}$ (250MHz; $\left.\mathrm{CD}_{3} \mathrm{OD}\right)$ 3.36-3.69 (3H, m, 5, 6a, 6b-H), $3.77\left(1 \mathrm{H}, \mathrm{dd}, \mathrm{J}_{3-4} 7.0, \mathrm{~J}_{3-2} 5.0,3-\right.$ $\mathrm{H}), 4.24\left(1 \mathrm{H}, \mathrm{J}_{4-3}\right.$ 7.0, 4-H), $4.42\left(2 \mathrm{H}, \mathrm{q}, \mathrm{J}_{\mathrm{H}-\mathrm{F}} 8.5, \mathrm{CH}_{2} \mathrm{CF}_{3}\right), 4.60\left(1 \mathrm{H}, \mathrm{J}_{2-3} 5.5,2-\mathrm{H}\right)$, $5.42(1 \mathrm{H}, \mathrm{s}, 1-\mathrm{H}) ; \delta_{\mathrm{C}}\left(62 \mathrm{MHz} ; \mathrm{CD}_{3} \mathrm{OD}\right) 63.0\left(\mathrm{q},{ }^{2} \mathrm{~J}_{\mathrm{C}-\mathrm{F}} 36.6, \mathrm{CH}_{2} \mathrm{CF}_{3}\right), 64.0,69.7,70.4$, 72.4, 76.0, 101.5, $122.8\left(\mathrm{q}, \mathrm{J}_{\mathrm{C}-\mathrm{F}} 276.4, \mathrm{CF}_{3}\right) ; \delta_{\mathrm{F}}\left(\mathrm{CD}_{3} \mathrm{OD}\right)-78.54 ; \mathrm{m} / \mathrm{z}(\mathrm{FAB}) 381$ $\left(\mathrm{MK}^{+}\right)$.

Methyl $\alpha$-D-glucopyranoside 2-(2',2',2'-trifluoroethyl sulfate) (49).

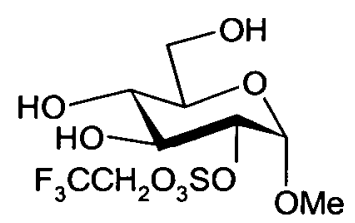

(49)

To a solution of (43) $(100 \mathrm{mg}, 0.23 \mathrm{mmol})$ and ethyl acetate $\left(2 \mathrm{~cm}^{3}\right)$ in acetic acid $\left(8 \mathrm{~cm}^{3}\right)$ was added $(5 \%)$ palladium on charcoal $(50 \mathrm{mg})$. The mixture was stirred under hydrogen for $24 \mathrm{hrs}$. The reaction was filtered and added to sat. potassium 
bicarbonate soln. $\left(15 \mathrm{~cm}^{3}\right)$, the product was then extracted into ethyl acetate (3x $10 \mathrm{~cm}^{3}$ ). The combined organic layers were dried with $\mathrm{MgSO}_{4}$ and the solvent evaporated under reduced pressure. Column chromatography (diethyl ether then ethyl acetate) gave starting material $(59 \mathrm{mg})$ and (49) $(24 \mathrm{mg}, 73 \%)$ as a colourless oil; $[\alpha]_{\mathrm{D}}^{16}+5.3$ (c 0.013 in $\left.\mathrm{MeOH}\right) ; v_{\max } / \mathrm{cm}^{-1} 3490,2930,1240 ; \delta_{\mathrm{H}}\left(250 \mathrm{MHz} ; \mathrm{CD}_{3} \mathrm{OD}\right)$ $3.46\left(1 \mathrm{H}, \mathrm{dd}, \mathrm{J}_{3-2} 10.0, \mathrm{~J}_{3-4} 8.5,3-\mathrm{H}\right), 3.63\left(1 \mathrm{H}, \mathrm{ddd}, \mathrm{J}_{5-4} 10.0, \mathrm{~J}_{5-6 \mathrm{a}} 5.5, \mathrm{~J}_{5-6 \mathrm{~b}} 2.0,5-\mathrm{H}\right)$, $3.77\left(1 \mathrm{H}, \mathrm{dd}, \mathrm{J}_{6 \mathrm{a}-6 \mathrm{~b}} 12.0, \mathrm{~J}_{6 \mathrm{a}-5} 5.5,6 \mathrm{a}-\mathrm{H}\right), 3.90\left(1 \mathrm{H}, \mathrm{dd}, \mathrm{J}_{4-5} 10.0, \mathrm{~J}_{4-3} 8.5,4-\mathrm{H}\right), 3.91$ $\left(1 \mathrm{H}, \mathrm{dd}, \mathrm{J}_{6 \mathrm{~b}-6 \mathrm{a}} 12.0, \mathrm{~J}_{6 \mathrm{~b}-5} 2.0,6 \mathrm{~b}-\mathrm{H}\right), 4.43\left(1 \mathrm{H}, \mathrm{dd}, \mathrm{J}_{2-3} 10.0, \mathrm{~J}_{2-1} 3.5,2-\mathrm{H}\right), 4.85(1 \mathrm{H}, \mathrm{dq}$, $\left.\mathrm{J}_{\mathrm{H}-\mathrm{H}} 12.0, \mathrm{~J}_{\mathrm{H}-\mathrm{F}} 8.0, \mathrm{CH}_{2} \mathrm{CF}_{3}\right), 4.98\left(1 \mathrm{H}, \mathrm{dq}, \mathrm{J}_{\mathrm{H}-\mathrm{H}} 12.0, \mathrm{~J}_{\mathrm{H}-\mathrm{F}} 8.0, \mathrm{CH}_{2} \mathrm{CF}_{3}\right), 5.08\left(1 \mathrm{H}, \mathrm{d}, \mathrm{J}_{1-}\right.$ $\left.{ }_{2} 3.5,1-\mathrm{H}\right) ; \delta_{\mathrm{C}}\left(62 \mathrm{MHz} ; \mathrm{CD}_{3} \mathrm{OD}\right) 53.7(\mathrm{CH}), 60.3\left(\mathrm{CH}_{2}\right), 66.2\left(\mathrm{q},{ }^{2} \mathrm{~J}_{\mathrm{C} \cdot \mathrm{F}} 37.9, \mathrm{CH}_{2} \mathrm{CF}_{3}\right)$, $70.0(\mathrm{CH}), 71.5(\mathrm{CH}), 82.7(\mathrm{CH}), 96.0(\mathrm{CH}), 121.6\left(\mathrm{q}, \mathrm{J}_{\mathrm{C}-\mathrm{F}} 276.4, \mathrm{CF}_{3}\right) ; \delta_{\mathrm{F}}(250 \mathrm{MHz}$; $\left.\mathrm{CD}_{3} \mathrm{OD}\right)-73.87 ; \mathrm{m} / \mathrm{z}$ (Electrospray) $379\left(\mathrm{MNa}^{+}\right),(\mathrm{FAB}) \mathrm{C}_{9} \mathrm{H}_{16} \mathrm{~F}_{3} \mathrm{O}_{9} \mathrm{~S}$ requires 357.04671, Found 357.04544 (dev. -3.57).

General procedure for the reaction of (32) with ethanethiol in the presence of various Lewis acids.

To a solution of (32) $(10 \mathrm{mg}, 19 \mu \mathrm{mol})$, ethanethiol $\left(0.2 \mathrm{~cm}^{3}\right)$ in dichloromethane $\left(1 \mathrm{~cm}^{3}\right)$ was added a Lewis acid $(20 \mu \mathrm{mol})$. The mixtures were then stirred at roon temperature for $6 \mathrm{~h}$. The reactions were monitored over this time period by TLC ( $4: 1$ diethyl ether : hexane).

General procedure for the reaction of (29) with thiophenol in the presence of Lewis acids.

To a solution of (29) $(255 \mathrm{mg}, 0.5 \mathrm{mmol})$, thiophenol $(66 \mathrm{mg}, 0.6 \mathrm{mmol})$ and $(4 \AA)$ molecular sieves in dichloromethane $\left(20 \mathrm{~cm}^{3}\right)$ was added the Lewis acid $(0.5 \mathrm{mmol})$. The reactions were monitored by TLC (3:2 diethyl ether :hexane). 
Phenyl 2,3,4-tri-O-acetyl-6-deoxy-6-thiophenyl- $\beta$-D-thioglucoside (51).

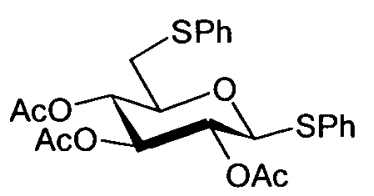

(51)

To a suspension of (29) $(25 \mathrm{mg}, 0.5 \mathrm{mml})$, thiophenol $(66 \mathrm{mg}, 0.6 \mathrm{mmol})$ and powdered molecular sieves $(4 \AA)(100 \mathrm{mg})$ in dichloromethane $\left(20 \mathrm{~cm}^{3}\right)$ was added zirconium tetrachloride $(116 \mathrm{mg}, 0.5 \mathrm{mmol})$ the mixture refluxed for $48 \mathrm{~h}$. Water $\left(5 \mathrm{~cm}^{3}\right)$ was added and the product extracted with diethyl ether $\left(3 \times 10 \mathrm{~cm}^{3}\right)$ the combined organic layers were dried with $\mathrm{MgSO}_{4}$ and the solvent evaporated under reduced pressure to give a colourless sold. The product was purified by column chromatography $(3: 2$ diethyl ether : hexane) to give (51) $(115 \mathrm{mg}, 47 \%)$ as a colourless oil; $[\alpha]_{\mathrm{D}}{ }^{16}+82.3(c$ 0.053 in $\left.\mathrm{CHCl}_{3}\right) ; \delta_{\mathrm{H}}\left(250 \mathrm{MHz} ; \mathrm{CDCl}_{3}\right) 1.97(3 \mathrm{H}, \mathrm{s}, \mathrm{OAc}), 2.01(3 \mathrm{H}, \mathrm{s}, \mathrm{OAc}), 2.07$ (3H, s, OAc), 2.96-3.17 (2H, m, 6a, 6b-H), 3.58-3.64 (1H, m, 5-H), $4.68(1 \mathrm{H}, \mathrm{d}, \mathrm{J}$ 10.0, 1-H), 4.92-5.22 (3H, m, 2,3,4-H), 7.15-7.42 (8H, m, Arom.) 7.45 (2H, m, Arom.); m/z (Electrospray) 381 (M-SPh) $491\left(\mathrm{MH}^{+}\right)$.

\section{Attempted conversion of the anomeric acetate of (32) to the bromide.}

Using the procedure published by van Boom and co-workers ${ }^{144}$. To a solution of (32) $(200 \mathrm{mg}, 0.4 \mathrm{mmol})$ and phosphorus tribromide $(65 \mathrm{mg}, 0.5 \mathrm{mmol})$ in acetic anhydride $\left(10 \mathrm{~cm}^{3}\right)$ at $0^{\circ} \mathrm{C}$ was added $(70 \%)$ perchloric acid $\left(0.2 \mathrm{~cm}^{3}\right)$. The mixture was allowed to warm to room temperature over $24 \mathrm{~h}$. However no reaction was observed by TLC (2:1 diethyl ether : hexane).

\section{3,4,6-Tri-O-acetyl-D-glucose 2 -(2',2',2'-trifluoroethyl sulfate) (52).}

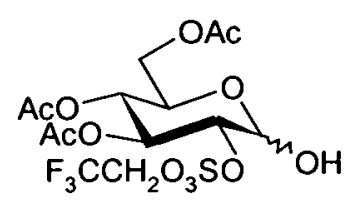

(52)

To a solution of (32) $(250 \mathrm{mg}, 0.5 \mathrm{mmol})$ in methanol $\left(15 \mathrm{~cm}^{3}\right)$ at $-12^{\circ} \mathrm{C}$ was added a solution of sodium methoxide $(26 \mathrm{mg}, 0.5 \mathrm{mmol})$. After $30 \mathrm{~min}$ dil. Citric acid soln. 
$(0.5 \mathrm{M})\left(10 \mathrm{~cm}^{3}\right)$ was added and the mixture allowed to warm to room temperature. The product was extracted with diethyl ether $\left(3 \times 20 \mathrm{~cm}^{3}\right)$, the combined organic layers were washed with water $\left(20 \mathrm{~cm}^{3}\right)$ and dried with $\mathrm{MgSO}_{4}$. The solvent was then evaporated under reduced pressure to give a colourless oil. The product was purified by column chromatography (3:2 diethyl ether: hexane) to give (52) $(130 \mathrm{mg}, 57 \%)$ as a colourless gum; $[\alpha]_{\mathrm{D}}^{16}+20.9\left(c 0.0165\right.$ in $\left.\mathrm{CHCl}_{3}\right) ; v_{\max } / \mathrm{cm}^{-1} 3479,2916,1747$, $1971,1224,1015 ; \delta_{\mathrm{H}}\left(250 \mathrm{MHz} ; \mathrm{CD}_{3} \mathrm{OD}\right) 2.06(3 \mathrm{H}, \mathrm{s}, \mathrm{OAc}), 2.08(3 \mathrm{H}, \mathrm{s}, \mathrm{OAc}), 2.13$ $(3 \mathrm{H}, \mathrm{s}, \mathrm{OAc}), 3.61(1 \mathrm{H}, \mathrm{bs}, \mathrm{OH}), 4.08-4.32(3 \mathrm{H}, \mathrm{m}, 5,6 \mathrm{a}, 6 \mathrm{~b}-\mathrm{H}), 4.51\left(1 \mathrm{H}, \mathrm{dq}, \mathrm{J}_{\mathrm{H}-\mathrm{H}}\right.$ $\left.11.0, \mathrm{H}_{\mathrm{H}-\mathrm{F}} 7.5, \mathrm{CH}_{2} \mathrm{CF}_{3}\right), 4.60\left(1 \mathrm{H}, \mathrm{dq}, \mathrm{J}_{\mathrm{H}-\mathrm{H}} 11.0, \mathrm{H}_{\mathrm{H}-\mathrm{F}} 7.5, \mathrm{CH}_{2} \mathrm{CF}_{3}\right), 4.69\left(1 \mathrm{H}, \mathrm{dd}, \mathrm{J}_{2-3}\right.$ 9.5, $\left.\mathrm{J}_{2-1} 3.5,2-\mathrm{H}\right), 5.10\left(1 \mathrm{H}, \mathrm{dd}, \mathrm{J}_{4-3} 10.0, \mathrm{~J}_{4-5} 10.0,4-\mathrm{H}\right), 5.59\left(1 \mathrm{H}, \mathrm{dd}, \mathrm{J}_{3-2} 10.0, \mathrm{~J}_{3-4}\right.$ $10.0,3-\mathrm{H}), 5.61\left(1 \mathrm{H}, \mathrm{d}, \mathrm{J}_{1-2} 3.5,1-\mathrm{H}\right) ; \delta_{\mathrm{F}}\left(250 \mathrm{MHz} ; \mathrm{CD}_{3} \mathrm{OD}\right)-71.97 ; \mathrm{m} / \mathrm{z}$ (Electrospray) $486\left(\mathrm{MNH}_{4}^{+}\right)$, (FAB) $469.06268\left(\mathrm{MH}^{+}\right) \mathrm{C}_{14} \mathrm{H}_{20} \mathrm{~F}_{3} \mathrm{O}_{12} \mathrm{~S}\left(\mathrm{MH}^{+}\right.$requires 469.06276 (dev. -0.17).

\section{Reaction of (52) with trichloroacetonitrile.}

A mixture of (52) $(200 \mathrm{mg}, 0.43 \mathrm{mmol})$, trichloroacetonitrile $(200 \mathrm{mg}, 1.38 \mathrm{mmol})$ potassium carbonate $(150 \mathrm{mg}, 1.5 \mathrm{mmol})$ in dichloromethane $\left(2 \mathrm{~cm}^{3}\right)$ was stirred at room temperature for $2 \mathrm{~h}$. TLC (3:2 diethyl ether : hexane) showed no reaction and MS (electrospray) of the crude mixture showed only peaks associated with the starting materials.

\section{2,3,4-Tri- $O$-acetyl-1,6-anhydro glucopyranose ${ }^{144}(53)$.}

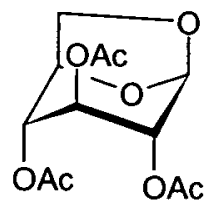

(53)

A suspension of (29) $(100 \mathrm{mg}, 0.2 \mathrm{mmol})$ and ammonium carbonate $(30 \mathrm{mg}, 0.4 \mathrm{mmol})$ in DMF $\left(1 \mathrm{~cm}^{3}\right)$ was stirred at room temperature for $16 \mathrm{~h}$. Water $\left(10 \mathrm{~cm}^{3}\right)$ was added and the product extracted into ethyl acetate $\left(3 \times 10 \mathrm{~cm}^{3}\right)$ and the combined organic layers were dried with $\mathrm{MgSO}_{4}$ and the solvent evaporated under reduced pressure to give an opaque oil. The product was purified by column chromatography ( $7: 3$ diethyl 
ether : hexane) to give (53) $(37 \mathrm{mg}, 66 \%)$ as a colourless gum; mp $107-109^{\circ} \mathrm{C}$ (from ethyl acetate) (lit., ${ }^{144}$ 108-109); $\delta_{\mathrm{H}}(250 \mathrm{MHz} ; \mathrm{CDCl} 3) 2.07(3 \mathrm{H}, \mathrm{s}, \mathrm{OAc}), 2.11(3 \mathrm{H}, \mathrm{s}$, OAc), 2.13 (3H, s, OAc), 3.77 (1H, dd, J 8.1, J 5.5, 6a-H), 4.08 (1H, dd, J 8.0, J 1.0, 6b-H), 4.56-4.60 (3H, m, 2,4,5-H), 4.81 (1H, m, 3-H), 5.42 (1H, bs, 1-H); (Lit., 148 $2.12(3 \mathrm{H}, \mathrm{s}, \mathrm{OAc}), 2.16(3 \mathrm{H}, \mathrm{s}, \mathrm{OAc}), 2.18$ (3H, s, OAc), 3.84 (1H, dd, J 6.1, J 7.8), $4.03(1 \mathrm{H}, \mathrm{dd}, \mathrm{J} 7.8, \mathrm{~J} 1.0), 4.60-4.65(3 \mathrm{H}, \mathrm{m}), 4.86(1 \mathrm{H}, \mathrm{m}), 5.43(1 \mathrm{H}, \mathrm{bs}) ; \mathrm{m} / \mathrm{z}$ (Electrospray) $279\left(\mathrm{MNa}^{+}\right)$.

\section{Phenyl 3,4,6-tri- $O$-acetyl- $\alpha$-D-thioglucopyranoside $\quad 2$-(2',2',2'-trifluoroethyl sulfate) (54).}

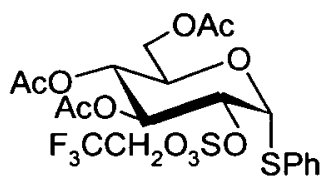

(54)

A solution of (58) $(259 \mathrm{mg}, 0.5 \mathrm{mmol})$ and sulfur trioxide pyridine complex $(530 \mathrm{mg}$, $3.3 \mathrm{mmole})$ in acetonitrile $\left(20 \mathrm{~cm}^{3}\right)$ was heated at $80^{\circ} \mathrm{C}$ for $2 \mathrm{~h}$. The mixture was cooled to room temperature and a solution of (24) $\left(20 \mathrm{~cm}^{3}\right)$ and citric acid monohydrate (1 $\left.\mathrm{g}\right)$ were added and the mixture stirred at room temperature for $16 \mathrm{~h}$. The solvent was then evaporated under reduced pressure and the resulting oil dissolved in water. The product was extracted with diethyl ether $\left(3 \times 25 \mathrm{~cm}^{3}\right)$ and the combined organic layers were, washed with sat. sodium bicarbonate soln. $\left(25 \mathrm{~cm}^{3}\right)$, dried with $\mathrm{MgSO}_{4}$ and the solvent was evaporated under reduced pressure to give a colourless solid. The product was purified by column chromatography (1:1 diethyl ether : hexane) to give (54) $(242 \mathrm{mg}, 67 \%)$ as a colourless solid; $\mathrm{mp} 110-112^{\circ} \mathrm{C}$ (from hexane); $[\alpha]_{\mathrm{D}}{ }^{16}+69.7$ (c $\left.0.0075 \mathrm{CHCl}_{3}\right) ; v_{\max } / \mathrm{cm}^{-1} 2916,1747,1419,1239,1041,878,741 ; \delta_{\mathrm{H}}(250 \mathrm{MHz}$; $\left.\mathrm{CDCl}_{3}\right) 2.03(3 \mathrm{H}, \mathrm{s}, \mathrm{OAc}), 2.05(3 \mathrm{H}, \mathrm{s}, \mathrm{OAc}), 2.07(3 \mathrm{H}, \mathrm{s}, \mathrm{OAc}), 4.05\left(1 \mathrm{H}, \mathrm{dd}, \mathrm{J}_{6 \mathrm{a}-6 \mathrm{~b}}\right.$ $\left.12.5, \mathrm{~J}_{6 \mathrm{a}-5} 2.5,6 \mathrm{a}-\mathrm{H}\right), 4.27\left(1 \mathrm{H}, \mathrm{dd}, \mathrm{J}_{6 \mathrm{~b}-6 \mathrm{a}} 12.5, \mathrm{~J}_{6 \mathrm{~b}-5} 5.0,6 \mathrm{~b}-\mathrm{H}\right), 4.58\left(2 \mathrm{H}, \mathrm{q}, \mathrm{J}_{\mathrm{H}-\mathrm{F}} 7.5\right.$, $\left.\mathrm{CH}_{2} \mathrm{CF}_{3}\right), 4.61\left(1 \mathrm{H}, \mathrm{ddd}, \mathrm{J}_{5-4} 10.0, \mathrm{~J}_{5-6 \mathrm{a}} 5.0, \mathrm{~J}_{5-6 \mathrm{~b}} 2.5,5-\mathrm{H}\right), 4.97\left(1 \mathrm{H}, \mathrm{dd}, \mathrm{J}_{2-3} 10.0, \mathrm{~J}_{2-1}\right.$ 6.0, 2-H), $5.08\left(1 \mathrm{H}, \mathrm{dd}, \mathrm{J}_{3-2} 10.0, \mathrm{~J}_{3-4} 9.0,3-\mathrm{H}\right), 5.42\left(1 \mathrm{H}, \mathrm{dd}, \mathrm{J}_{4-5} 10.0, \mathrm{~J}_{4-5} 9.0,4-\mathrm{H}\right)$, $5.84\left(1 \mathrm{H}, \mathrm{d}, \mathrm{J}_{1-2}\right.$ 6.0, 1-H), 7.31-7.35 (3H, m, Arom.), 7.43-7.49 (2H, m, Arom.); $\delta_{\mathrm{C}}$ $\left(62 \mathrm{MHz} ; \mathrm{CDCl}_{3}\right) 20.3$ (OAc), 20.3 (OAc), 20.4 (OAc), $61.4(6-\mathrm{C}), 67.2$ (q, ${ }^{2} \mathrm{~J}_{\mathrm{C}-\mathrm{F}} 38.7$, 
$\left.\mathrm{CH}_{2} \mathrm{CF}_{3}\right), 68.1,68.2,69.6,78.8,85.0,121.4\left(\mathrm{q}, \mathrm{J}_{\mathrm{C}-\mathrm{F}} 277.4, \mathrm{CF}_{3}\right), 128.3(1 \times$ Arom), 129.3 ( $2 \times$ Arom), 131.1 (Arom), 132.1 ( $2 \times$ Arom), $169.3(\mathrm{C}=0), 169.6(\mathrm{C}=0)$, $170.3(\mathrm{C}=\mathrm{O}) ; \delta_{\mathrm{F}}\left(250 \mathrm{MHz} ; \mathrm{CDCl}_{3}\right)-74.00 ; \mathrm{m} / \mathrm{z}(\mathrm{APCl}) 451(\mathrm{M}-\mathrm{SPh}), 578\left(\mathrm{MNH}_{4}{ }^{+}\right)$.

\section{Attempted glycosidation of (54) with ethanol.}

A mixture of (54) $(56 \mathrm{mg}, 0.1 \mathrm{mmol})$, ethanol $(23 \mathrm{mg}, 0.5 \mathrm{mmol})$ and powdered $(4 \AA)$ molecular sieves $(\sim 50 \mathrm{mg})$ in dichloromethane $\left(5 \mathrm{~cm}^{3}\right)$ was stirred at room temperature for $10 \mathrm{~min}$ under an argon environment. To the mixture was added either $N$-iodosuccinamide $(54 \mathrm{mg}, 0.13 \mathrm{mmol})$ or $N$-bromosuccinamide $(43 \mathrm{mg}, 0.13 \mathrm{mmol}$ ) and trifluoromethyl sulfonic acid (30mg, $0.1 \mathrm{mmol}$ ) depending on the reaction conditions stated.

Phenyl 3,4-di- $O$-acetyl-2-deoxy-2- $N$-pthalimido- $\beta$-D-thiogalactopyranoside 6 $\left(2^{\prime}, 2^{\prime}, 2^{\prime}\right.$-trifluoroethyl sulfate) (55).

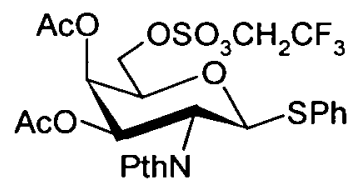

(55)

A solution of (65) (200mg, 0.4mmol) and sulfur trioxide complex (328mg, 2.0mmol) in acetonitrile $\left(10 \mathrm{~cm}^{3}\right)$ was heated at $80^{\circ} \mathrm{C}$ for $2 \mathrm{~h}$. The solution was then cooled to room temperature and a solution of (24) $\left(20 \mathrm{~cm}^{3}\right)$ was added followed by citric acid monohydrate $(1 \mathrm{~g})$. The mixture was stirred for $20 \mathrm{~h}$ then the solvent was removed and the resulting solid purified by column chromatography (1:1 ethyl acetate : hexane) to give (55) $(158 \mathrm{mg}, 59 \%)$ as a colourless solid; $\mathrm{mp} 49-51^{\circ} \mathrm{C}$ (from hexane); $[\alpha]_{\mathrm{D}}{ }^{16}$ +9.22 (c 0.009 in $\left.\mathrm{CHCl}_{3}\right) ; v_{\max } / \mathrm{cm}^{-1} 2974,1716,1386,1224,1045,936,877 ; \delta_{\mathrm{H}}$ $\left(250 \mathrm{MHz} ; \mathrm{CDCl}_{3}\right) 1.91(3 \mathrm{H}, \mathrm{s}, \mathrm{OAc}), 2.07(3 \mathrm{H}, \mathrm{s}, \mathrm{OAc}), 4.12-4.70(6 \mathrm{H}, \mathrm{m}, 2,5,6 \mathrm{a}$, 6b, $\left.\mathrm{CH}_{2} \mathrm{CF}_{3}\right), 5.27(1 \mathrm{H}, \mathrm{d}, \mathrm{J}$ 3.0, 4-H), $5.69(1 \mathrm{H}, \mathrm{d}, \mathrm{J} 10.5,1-\mathrm{H}), 5.57(1 \mathrm{H}, \mathrm{dd}, \mathrm{J} 11.0$, J 3.0, 3-H), 7.26-7.51 (5H, m, Arom.), 7.73-7.88 (4H, m, Arom.); $\delta_{\mathrm{C}}(62 \mathrm{MHz}$; $\left.\mathrm{CDCl}_{3}\right) 20.1$ (OAc), $20.4(\mathrm{OAc}), 49.2,60.9,67.0\left(\mathrm{q}, \mathrm{J}_{\mathrm{C}-\mathrm{F}} 38.6, \mathrm{CH}_{2} \mathrm{CF}_{3}\right), 68.2,73.8$, $79.7,83.3,121.5\left(\mathrm{q}, \mathrm{J}_{\mathrm{C}-\mathrm{F}} 277.5, \mathrm{CF}_{3}\right), 123.6,123.8,128.6,128.8(2 \times \mathrm{C}), 129.0$, $130.2,133.2,133.6(2 \times C), 134.4(2 \times C), 167.8(C=0), 169.6(C=O), 170.1(C=0)$; 
$\delta_{\mathrm{F}}\left(250 \mathrm{MHz} ; \mathrm{CDCl}_{3}\right)-74.13 ; \mathrm{m} / \mathrm{z}(\mathrm{APCI}) 665\left(\mathrm{MNH}_{4}^{+}\right),(\mathrm{FAB}) \mathrm{C}_{26} \mathrm{H}_{25} \mathrm{~F}_{3} \mathrm{NO}_{11} \mathrm{~S}_{2}$ $\left(\mathrm{MH}^{+}\right)$requires 648.08212, found 648.08038 (dev. -2.68).

\section{3,4,6-Tri- $O$-acetyl-2-trichloroacetyl- $\beta$-D-glucopyranosyl chloride ${ }^{149}$ (56).}

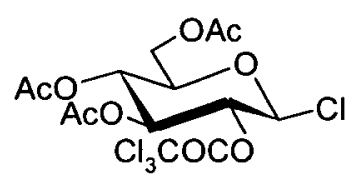

(56)

A mixture of penta- $O$-acetyl- $\beta$-D-glucopyranose $(20.0 \mathrm{~g}, 52 \mathrm{mmol})$, and phosphorous pentachloride $(60.0 \mathrm{~g}, 28 \mathrm{mmol})$ was heated at reflux (oil bath temperature of $130^{\circ} \mathrm{C}$ ) for $2 \mathrm{~h}$. Volatile by-products were removed by vacuum distillation $(10 \mathrm{mmHg})$ initially at room temperature and then by warming to $100^{\circ} \mathrm{C}$. The resulting yellow syrup was dissolved in water $\left(100 \mathrm{~cm}^{3}\right)$ and extracted with dichloromethane $(3 \mathrm{x}$ $100 \mathrm{~cm}^{3}$ ). The combined organic layers were washed with water and then dried with $\mathrm{MgSO}_{4}$. The solvent was evaporated under reduced pressure to give a yellow oil which was dissolved in diethyl ether $\left(60 \mathrm{~cm}^{3}\right)$ and allowed to stand at $-28^{\circ} \mathrm{C}$ for $16 \mathrm{~h}$. The resulting crystals were filtered to give (56) (4.94g). The filtrate was evaporated under reduced pressure to give a yellow oil that was purified by column chromatography (3:2 hexane : diethyl ether) to give a further portion of (56) $(7.79 \mathrm{~g}$, total yield 53\%) as a colourless solid; $\delta_{\mathrm{H}}\left(250 \mathrm{MHz} ; \mathrm{CDCl}_{3}\right) 2.00(3 \mathrm{H}, \mathrm{s}, \mathrm{OAc}), 2.02$ ( $3 \mathrm{H}, \mathrm{s}, \mathrm{OAc}), 2.11(3 \mathrm{H}, \mathrm{s}, \mathrm{OAc}), 3.85\left(1 \mathrm{H}, \mathrm{ddd}, \mathrm{J}_{5-4} 9.0, \mathrm{~J}_{5-6 \mathrm{a}} 4.5, \mathrm{~J}_{5-6 \mathrm{~b}} 2.5,5-\mathrm{H}\right), 4.17$ $\left(1 \mathrm{H}, \mathrm{dd}, \mathrm{J}_{6 \mathrm{~b}-6 \mathrm{a}} 12.5, \mathrm{~J}_{6 \mathrm{~b}-5} 2.5,6 \mathrm{~b}-\mathrm{H}\right), 4.29\left(1 \mathrm{H}, \mathrm{dd}, \mathrm{J}_{6 \mathrm{a}-6 \mathrm{~b}} 12.5, \mathrm{~J}_{6 \mathrm{a}-5} 4.5,6 \mathrm{a}-\mathrm{H}\right), 5.17(1 \mathrm{H}$, dd, $\left.\mathrm{J}_{3-4} 9.0, \mathrm{~J}_{3-2} 9.0,3-\mathrm{H}\right), 5.2217\left(1 \mathrm{H}, \mathrm{dd}, \mathrm{J}_{4-3} 9.0, \mathrm{~J}_{4-5} 9.0,4-\mathrm{H}\right), 5.3617\left(1 \mathrm{H}, \mathrm{dd}, \mathrm{J}_{2-3}\right.$ 9.0, $\left.\mathrm{J}_{2-1} 9.0,2-\mathrm{H}\right), 5.39\left(1 \mathrm{H}, \mathrm{d}, \mathrm{J}_{1-2} 8.0,1-\mathrm{H}\right) ; \delta_{\mathrm{C}}\left(62 \mathrm{MHz} ; \mathrm{CDCl}_{3}\right) 20.2(\mathrm{OAc}), 20.3$ (OAc), $20.5(\mathrm{OAc}), 61.2(6-\mathrm{C}), 67.4(\mathrm{CH}), 71.7(\mathrm{CH}), 75.6(\mathrm{CH}), 77.0(\mathrm{CH}), 86.3$ $(\mathrm{CH}), 160.2\left(\mathrm{O}=\mathrm{CCCl}_{3}\right), 169.1(\mathrm{C}=\mathrm{O}), 169.4(\mathrm{C}=\mathrm{O}), 170.4(\mathrm{C}=\mathrm{O})$. 
3,4,6-Tri- $O$-acetyl- $\beta$-D-galactopyranosyl chloride ${ }^{150}(57)$.

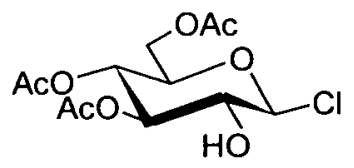

(57)

To a saturated solution of ammonia gas in diethyl ether at $0^{\circ} \mathrm{C}$ was added (56) $(9.73 \mathrm{~g}$, $21 \mathrm{mmol}$ ). The mixture was stirred for $8 \mathrm{~min}$ then the mixture was filtered and the crystals washed with cold ether the crystals were dried under vacuum to give (57) $(5.85 \mathrm{~g}, 86 \%)$ as a colourless solid. The compound was used in the synthesis of (58) without further purification or characterisation.

Phenyl 3,4,6-tri- $O$-acetyl- $\alpha$-D-thiogalactopyranoside 152 (58) and Phenyl 3,4,6tri- $O$-acetyl- $\beta$-D-thiogalactopyranoside 192 (107).

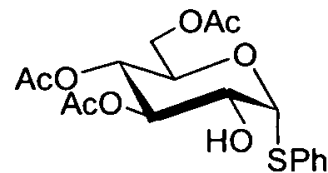

(58)

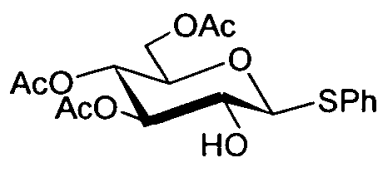

(107)

Potassium metal $(0.30 \mathrm{~g}, 7.7 \mathrm{mmol})$ was added to methanol $\left(10 \mathrm{~cm}^{3}\right)$ at $0^{\circ} \mathrm{C}$. Once dissolved the solution was cooled to $-78^{\circ} \mathrm{C}$ and thiophenol $(8.47 \mathrm{~g}, 77.0 \mathrm{mmol})$ was added. After $5 \mathrm{~min}$ a solution of (57) $(2.50 \mathrm{~g}, 7.7 \mathrm{mmol})$ in methanol $\left(20 \mathrm{~cm}^{3}\right)$ was added and the mixture was allowed to warm to room temperature over $16 \mathrm{~h}$. The solvent was evaporated under reduced pressure and the resulting oil was purified by column chromatography (7:3 diethyl ether : hexane) to give (58) as a colourless oil $(1.17 \mathrm{~g}, 38 \%) ;[\alpha]_{\mathrm{D}}{ }^{16}+179.9^{\circ}\left(c 0.0225, \mathrm{CHCl}_{3}\right)\left(\mathrm{lit}^{152}[\alpha]_{\mathrm{D}}{ }^{20}+180^{\circ}\right) ; v_{\max } / \mathrm{cm}^{-1} 3357$, $2847,1738,1464,1243,1042,863,722 ; \delta_{\mathrm{H}}\left(250 \mathrm{MHz} ; \mathrm{CDCl}_{3}\right) 2.03(3 \mathrm{H}, \mathrm{s}, \mathrm{OAc})$, $2.04(3 \mathrm{H}, \mathrm{s}, \mathrm{OAc}), 2.07(3 \mathrm{H}, \mathrm{s}, \mathrm{OAc}), 2.58\left(1 \mathrm{H}, \mathrm{d}, \mathrm{J}_{\mathrm{OH}-2} 9.5,2-\mathrm{OH}\right), 4.01\left(1 \mathrm{H}, \mathrm{ddd}, \mathrm{J}_{2-}\right.$ он $\left.9.5, \mathrm{~J}_{2-3} 9.5, \mathrm{~J}_{2-1} 5.5,2-\mathrm{H}\right), 4.06\left(1 \mathrm{H}, \mathrm{dd}, \mathrm{J}_{6 \mathrm{a}-6 \mathrm{~b}} 12.5, \mathrm{~J}_{6 \mathrm{a}-5} 2.5,6 \mathrm{a}-\mathrm{H}\right), 4.30\left(1 \mathrm{H}, \mathrm{dd}, \mathrm{J}_{6 \mathrm{~b}-}\right.$ $\left.{ }_{6 \mathrm{a}} 12.5, \mathrm{~J}_{6 \mathrm{~b}-5} 5.0,5-\mathrm{H}\right), 4.50\left(1 \mathrm{H}, \mathrm{ddd}, \mathrm{J}_{5-4} 9.5, \mathrm{~J}_{5-6 \mathrm{~b}} 5.0, \mathrm{~J}_{5-6 \mathrm{a}} 2.5,5-\mathrm{H}\right), 5.02\left(1 \mathrm{H}, \mathrm{dd}, \mathrm{J}_{3-4}\right.$ 9.5, $\left.\mathrm{J}_{3-2} 9.5,3-\mathrm{H}\right), 5.11\left(1 \mathrm{H}, \mathrm{dd}, \mathrm{J}_{4-5} 9.5, \mathrm{~J}_{4-3} 9.5,4-\mathrm{H}\right), 5.62\left(1 \mathrm{H}, \mathrm{d}, \mathrm{J}_{1-2} 5.5,1-\mathrm{H}\right), 7.26-$ 7.32 (3H, m, Arom.), 7.43-7.51 (2H, m, Arom.); $\delta_{\mathrm{C}}\left(62 \mathrm{MHz} ; \mathrm{CDCl}_{3}\right) 20.5\left(2 \mathrm{x} \mathrm{CH}_{3}\right)$, $20.6\left(\mathrm{CH}_{3}\right), 61.8(6-\mathrm{C}), 67.8(\mathrm{CH}), 68.7(\mathrm{CH}), 70.5(\mathrm{CH}), 73.8(\mathrm{CH}), 90.3(\mathrm{CH})$, $127.8(\mathrm{CH}), 129.0(2 \times \mathrm{CH}), 131.8(2 \times \mathrm{CH}), 132.8(\mathrm{C}), 169.4(\mathrm{C}=\mathrm{O}), 170.4(\mathrm{C}=\mathrm{O})$, 
$170.9(\mathrm{C}=\mathrm{O}) ; \mathrm{m} / \mathrm{z}$ (Electrospray) $399\left(\mathrm{MH}^{+}\right), 421\left(\mathrm{MNa}^{+}\right)$. and (107) $(0.45 \mathrm{~g}, 14.5 \%)$ $v_{\max } / \mathrm{cm}^{-1} 3357,2847,1738,1464,1243,1042,863,722 ; \delta_{\mathrm{H}}\left(250 \mathrm{MHz} ; \mathrm{CDCl}_{3}\right) 2.03$ (3H, s, OAc), 2.07 (3H, s, OAc), 2.09 (3H, s, OAc), $2.51\left(1 \mathrm{H}, \mathrm{d}, \mathrm{J}_{\mathrm{OH}-2} 3.0, \mathrm{OH}\right), 3.50$ $\left(1 \mathrm{H}, \mathrm{ddd}, \mathrm{J}_{2-3} 10.0, \mathrm{~J}_{2-1} 10.0, \mathrm{~J}_{2-\mathrm{OH}} 3.0,2-\mathrm{H}\right), 3.74\left(1 \mathrm{H}, \mathrm{ddd}, \mathrm{J}_{5-4} 10.0, \mathrm{~J}_{5-6 \mathrm{~b}} 5.0, \mathrm{~J}_{5-6 \mathrm{a}} 2.5\right.$, $5-\mathrm{H}), 4.16\left(1 \mathrm{H}, \mathrm{dd}, \mathrm{J}_{6 \mathrm{a}-6 \mathrm{~b}} 125, \mathrm{~J}_{6 \mathrm{a}-5} 2.5,6 \mathrm{a}-\mathrm{H}\right), 4.25\left(1 \mathrm{H}, \mathrm{dd}, \mathrm{J}_{6 \mathrm{~b}-6 \mathrm{a}} 12.5, \mathrm{~J}_{6 \mathrm{~b}-5} 5.0,6 \mathrm{~b}-\mathrm{H}\right)$, $4.57\left(1 \mathrm{H}, \mathrm{d}, \mathrm{J}_{1-2} 10.0,1-\mathrm{H}\right), 4.98\left(1 \mathrm{H}, \mathrm{dd}, \mathrm{J}_{3-4} 10.0, \mathrm{~J}_{3-2} 10.0,3-\mathrm{H}\right), 5.15\left(1 \mathrm{H}, \mathrm{dd}, \mathrm{J}_{4-5}\right.$ 10.0, $\left.\mathrm{J}_{4-3} 10.0,4-\mathrm{H}\right), 7.30-7.36$ (3H, m, Arom.), 7.55-7.58 (2H, m, Arom.); m/z (Electrospray) $399\left(\mathrm{MH}^{+}\right), 421\left(\mathrm{MNa}^{+}\right)$.

\section{Phenyl 3,4,6-tri- $O$-acetyl- $\alpha$-D-glucosyl sulfoxide 2 -(2',2',2'-trifluoroethyl sulfate)} (59).

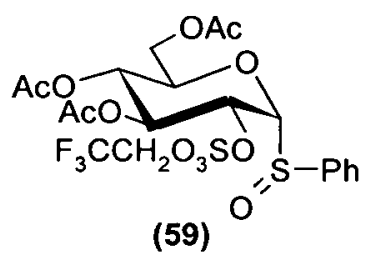

To a solution of (54) $(56 \mathrm{mg}, 0.1 \mathrm{mmol})$ in dichloromethane $\left(2 \mathrm{~cm}^{3}\right)$ at $-78^{\circ} \mathrm{C}$ was added a solution of meta-chloro perbenzoic acid (18mg of an $86 \%$ pure mixture with benzoic acid and water, $0.1 \mathrm{mmol})$ in dichloromethane $\left(0.5 \mathrm{~cm}^{3}\right)$. The mixture was allowed to warm to room temperature over $1 \mathrm{~h}$. Another solution of meta-chloro perbenzoic acid (9mg of an $86 \%$ pure mixture with benzoic acid and water, $0.05 \mathrm{mmol})$ in dichloromethane $\left(0.5 \mathrm{~cm}^{3}\right)$ was added. After a further $1 \mathrm{~h}$ water $\left(5 \mathrm{~cm}^{3}\right)$ was added. The product was extracted into dichloromethane $\left(3 \times 10 \mathrm{~cm}^{3}\right)$ and the combined organic layers were washed with $(2 \mathrm{~N})$ sodium bicarbonate soln. $\left(10 \mathrm{~cm}^{3}\right)$ and dried with $\mathrm{MgSO}_{4}$. The solvent was evaporated under reduced pressure to give a solid that was purified by column chromatography (7:3 diethyl ether : hexane) to give (59) $(38 \mathrm{mg}, 66 \%)$ as a colourless solid; $\mathrm{mp} 95-98^{\circ} \mathrm{C}$ (from hexane); $[\alpha]_{\mathrm{D}}{ }^{16}+21.5$ (c 0.0105 in $\mathrm{CHCl}_{3}$ ); (Found: $\mathrm{C}, 41.35 ; \mathrm{H}, 4.3 ; \mathrm{C}_{20} \mathrm{H}_{23} \mathrm{~F}_{3} \mathrm{O}_{12} \mathrm{~S}_{2}$ requires $\mathrm{C}, 41.6 ; \mathrm{H}$, $4.0 \%) ; v_{\max } / \mathrm{cm}^{-1} 2994,1746,1415,1206,1151,1042,874 ; \delta_{\mathrm{H}}\left(250 \mathrm{MHz} ; \mathrm{CDCl}_{3}\right) 1.97$ $(3 \mathrm{H}, \mathrm{s}, \mathrm{OAc}), 2.07(3 \mathrm{H}, \mathrm{s}, \mathrm{OAc}), 2.09(3 \mathrm{H}, \mathrm{s}, \mathrm{OAc}), 3.93\left(1 \mathrm{H}, \mathrm{dd}, \mathrm{J}_{6 \mathrm{a}-6 \mathrm{~b}} 12.5, \mathrm{~J}_{6 \mathrm{a}-5} 3.0\right.$, $6 \mathrm{a}-\mathrm{H}), 4.13\left(1 \mathrm{H}, \mathrm{dd}, \mathrm{J}_{6 \mathrm{~b}-6 \mathrm{a}} 12.5, \mathrm{~J}_{6 \mathrm{~b}-5} 6.0,6 \mathrm{~b}-\mathrm{H}\right), 4.25\left(1 \mathrm{H}, \mathrm{ddd}, \mathrm{J}_{5-4} 8.5, \mathrm{~J}_{5-6 \mathrm{~b}} 6.0, \mathrm{~J}_{5-6 \mathrm{a}}\right.$ $3.0,5-\mathrm{H}), 4.65\left(1 \mathrm{H}, \mathrm{dq}, \mathrm{J}_{\mathrm{H}-\mathrm{F}} 8.0, \mathrm{CH}_{2} \mathrm{CF}_{3}\right), 4.74\left(1 \mathrm{H}, \mathrm{d}, \mathrm{J}_{1-2} 4.5,1-\mathrm{H}\right), 4.84\left(1 \mathrm{H}, \mathrm{dq}, \mathrm{J}_{\mathrm{H}-}\right.$ 
F $\left.8.0, \mathrm{CH}_{2} \mathrm{CF}_{3}\right), 5.00\left(1 \mathrm{H}, \mathrm{dd}, \mathrm{J}_{4-5} 8.5, \mathrm{~J}_{4-3} 6.0,4-\mathrm{H}\right), 5.20\left(1 \mathrm{H}, \mathrm{dd}, \mathrm{J}_{2-3} 7.0, \mathrm{~J}_{2-1} 4.5,2-\right.$ $\mathrm{H}), 5.86\left(1 \mathrm{H}, \mathrm{dd}, \mathrm{J}_{3.2} 7.0, \mathrm{~J}_{3-4} 6.0,3-\mathrm{H}\right), 7.54-7.64(3 \mathrm{H}, \mathrm{m}$, Arom. $), 7.70-7.74(2 \mathrm{H}, \mathrm{m}$, Arom.); $\delta_{\mathrm{C}}\left(62 \mathrm{MHz} ; \mathrm{CDCl}_{3}\right) 20.3(\mathrm{OAc}), 20.4(2 \times \mathrm{OAc}), 61.0(6-\mathrm{C}), 67.0(\mathrm{CH}), 67.6$ $\left(\mathrm{q},{ }^{2} \mathrm{~J}_{\mathrm{C}-\mathrm{F}} 38.6, \mathrm{CH}_{2} \mathrm{CF}_{3}\right), 68.2(\mathrm{CH}), 73.4(\mathrm{CH}), 76.7(\mathrm{CH}), 90.7(\mathrm{CH}), 121.4\left(\mathrm{q}, \mathrm{J}_{\mathrm{C}-\mathrm{F}}\right.$ 277.4, $\mathrm{CF}_{3}$ ), 125.3 ( $2 \times \mathrm{CH}$ Arom.), 129.3 ( $2 \times \mathrm{CH}$ Arom.), 132.2 (CH, Arom.), 139.6 (c, Arom.), $168.8(\mathrm{C}=\mathrm{O}), 169.2(\mathrm{C}=\mathrm{O}), 170.0(\mathrm{C}=\mathrm{O}) ; . \delta_{\mathrm{F}}\left(250 \mathrm{MHz} ; \mathrm{CDCl}_{3}\right)-74.03$; $\mathrm{m} / \mathrm{z}$ (Electrospray) $577\left(\mathrm{MH}^{+}\right),(\mathrm{FAB}) 599.04826\left(\mathrm{MNa}^{+}, \mathrm{C}_{20} \mathrm{H}_{23} \mathrm{~F}_{3} \mathrm{O}_{12} \mathrm{SNa}\right.$ requires 599.04811 (dev. 0.26)).

1,2 Anhydro-3,4,6-tri- $O$-acetyl- $\alpha$-D-glucopyranose ${ }^{193}$ (61).

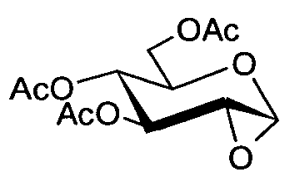

(61)

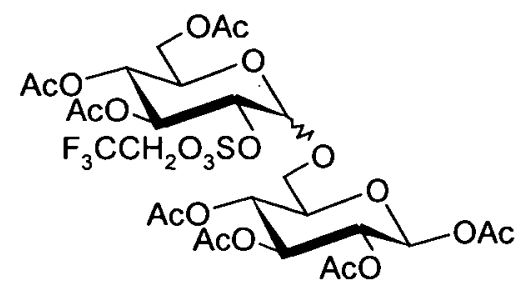

(60)

A solution of (59) (115mg, 0.2mmol) and 4-methyl 2,6-di-tert-butyl pyridine ( $82 \mathrm{mg}$, $0.4 \mathrm{mmol})$ in dichloromethane $\left(8 \mathrm{~cm}^{3}\right)$ was cooled to $-78^{\circ} \mathrm{C}$. Trifluoromethyl-sulfonic anhydride (46mg, $0.22 \mathrm{mmol}$ ) was added followed by a solution of tetra- $O$-acetyl glucose in dichloromethane $\left(2 \mathrm{~cm}^{3}\right)$. The mixture was stirred at $-78^{\circ} \mathrm{C}$ for $1 \mathrm{~h}$ before being warmed to room temperature. TLC (7:3 diethyl ether :hexane) indicated one new product. A sample of the crude reaction mixture was examined by MS (electrospray) and displayed peaks for (60) $821\left(\mathrm{MNa}^{+}\right)$and for (61) $311\left(\mathrm{MNa}^{+}\right)$. The solvent was evaporated under reduced pressure to give gum that was purified by column chromatography (7:3 diethyl ether : hexane) to give (61) (50mg, 86\%)as a gum; $v_{\max } / \mathrm{cm}^{-1} 2916,1738,1370,1230,1049,890 ; \delta_{\mathrm{H}}\left(200 \mathrm{MHz} ; \mathrm{CDCl}_{3}\right) 2.06(3 \mathrm{H}, \mathrm{s}$, OAc), $2.09(3 \mathrm{H}, \mathrm{s}, \mathrm{OAc}), 2.11(3 \mathrm{H}, \mathrm{s}, \mathrm{OAc}), 3.75\left(1 \mathrm{H}, \mathrm{dd}, \mathrm{J}_{6 \mathrm{~b}-6 \mathrm{a}} 8.0, \mathrm{~J} 5.5, \mathrm{H} 6 \mathrm{~b}-\mathrm{H}\right)$, $4.05\left(1 \mathrm{H}, \mathrm{dd}, \mathrm{J}_{6 \mathrm{a}-6 \mathrm{~b}} 8.0, \mathrm{~J} 1.0,6 \mathrm{a}-\mathrm{H}\right), 4.53-4.65(3 \mathrm{H}, \mathrm{m}, 3,4,5-\mathrm{H}), 4.79\left(1 \mathrm{H}, \mathrm{dd}, \mathrm{J}_{2-1}\right.$ 1.5, J 1.5, 2-H), $5.40\left(1 \mathrm{H}, \mathrm{d}, \mathrm{J}_{1-2} 1.5,1-\mathrm{H}\right) ; \delta_{\mathrm{C}}\left(62 \mathrm{MHz} ; \mathrm{CDCl}_{3}\right) 20.6\left(2 \mathrm{x} \mathrm{CH}_{3}\right), 20.7$ $\left(\mathrm{CH}_{3}\right), 65.1(\mathrm{C} 6), 68.8(\mathrm{CH}), 69.4(\mathrm{CH}), 70.0(\mathrm{CH}), 73.5(\mathrm{CH}), 98.9(\mathrm{C} 1), 168.8$ $(\mathrm{C}=\mathrm{O}), 169.4(\mathrm{C}=\mathrm{O}), 169.8(\mathrm{C}=\mathrm{O})\left(\right.$ lit. $\left.{ }^{193}\right) ; \mathrm{m} / \mathrm{z}$ (electrospray) $311\left(\mathrm{MNa}^{+}\right)$. 
Phenyl 3,4-di- $O$-acetyl-2-deoxy-2- $N$-pthalimido- $\beta$-D-thiogalactopyranoside (65).

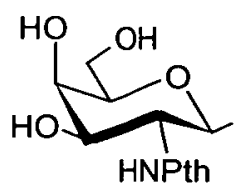

(62)

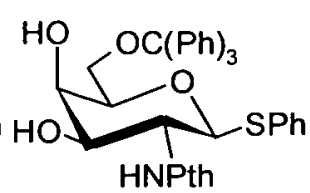

(63)

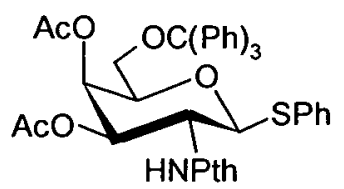

(64)

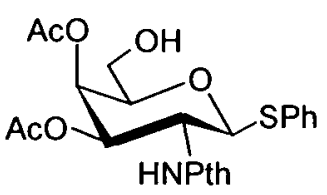

(65)

A solution of phenyl 2-deoxy-2-pthalimido- $\beta$-D-thiogalactopyranoside ${ }^{194}(401 \mathrm{mg}$, $1.0 \mathrm{mmol})$ and triphenyl methyl chloride $(696 \mathrm{mg}, 2.5 \mathrm{mmol})$ in pyridine $\left(5 \mathrm{~cm}^{3}\right)$ was heated at $50^{\circ} \mathrm{C}$ for $20 \mathrm{~h}$. After this time no starting material was visible by TLC (1:1 ethyl acetate : hexane) and there was a new less polar spot which was (63). The mixture was cooled to room temperature and acetic anhydride $\left(5 \mathrm{~cm}^{3}\right)$ was added. After $2 \mathrm{~h}$ (63) was not visible by TLC (1:1 ethyl acetate : hexane) and there was a new product spot which was (64). The solvent was evaporated under reduced pressure and replaced with $75 \%$ acetic acid $\left(10 \mathrm{~cm}^{3}\right)$. The mixture was then stirred at $50^{\circ} \mathrm{C}$ for a further $16 \mathrm{~h}$ after which time no (64) was visible by TLC. The solvent was evaporated under reduced pressure and the product purified by column chromatography (3:2 hexane: ethyl acetate) to give (65) (260mg, 54\% over 3 steps) as a colourless solid; $\mathrm{mp} 76-78^{\circ} \mathrm{C}$ (from hexane); $v_{\max } / \mathrm{cm}^{-1} 3041,2916,2847,1744$, $1716,1471,1232,1072,719 ; \delta_{\mathrm{H}}\left(250 \mathrm{MHz} \mathrm{CDCl}_{3}\right) 1.91(3 \mathrm{H}, \mathrm{s}, \mathrm{OAc}), 2.06(3 \mathrm{H}, \mathrm{s}$, OAc), 3.96 (1H, ddd, J 7.0, J 6.0, J 1.0, 5-H), 4.54 (1H, dd, J 3.0, J 1.0, 4-H), 4.26$4.41(2 \mathrm{H}, \mathrm{m}, 6 \mathrm{a}, 6 \mathrm{~b}-\mathrm{H}), 4.65\left(1 \mathrm{H}, \mathrm{dd}, \mathrm{J}_{2-3} 10.5, \mathrm{~J}_{2-1} 10.5,2-\mathrm{H}\right), 5.66\left(1 \mathrm{H}, \mathrm{d}, \mathrm{J}_{1-2} 10.5\right.$, 1-H), 5.67 (1H, dd, J $\mathrm{J}_{3-2} 10.5, \mathrm{~J}_{3-4}$ 3.0, 3-H), 7.21-7.29 (3H, m, Arom.), 7.30-7.50 (2H, m, Arom.), 7.70-7.82 (2H, m, Arom.), 7.83-7.88 (2H, m, Arom.); $\delta_{\mathrm{c}}(62 \mathrm{MHz}$; $\mathrm{CDCl}_{3}$ ) 20.5 (OAc), 20.6 (OAc), 49.7, 62.6, 66.6, 70.9, 75.8, 83.4, 123.5 (2 x CH), $127.9,128.7(4 \times \mathrm{C}), 132.4(4 \times \mathrm{C}), 134.2(\mathrm{C}), 167.1(\mathrm{C}=\mathrm{O}), 167.9(\mathrm{C}=0), 169.7$ $(\mathrm{C}=\mathrm{O}), 170.7(\mathrm{C}=\mathrm{O}) ; \mathrm{m} / \mathrm{z}(\mathrm{APCI}) 503\left(\mathrm{MNH}^{+}\right),(\mathrm{FAB}) \mathrm{C}_{24} \mathrm{H}_{24} \mathrm{NO}_{8} \mathrm{~S}\left(\mathrm{MH}^{+}\right)$requires 486.12226, found 486.12457 (dev. 4.75). 


\section{1;2,3;4-Di-O-isopropylidene-6-(3,4-di- $O$-acetyl-2-deoxy-2- $N$-pthalimido-D-} galactopyranosyl 6-(2',2',2'-trifluoroethyl sulfate))- $\alpha$-D-galactopyranose (66).

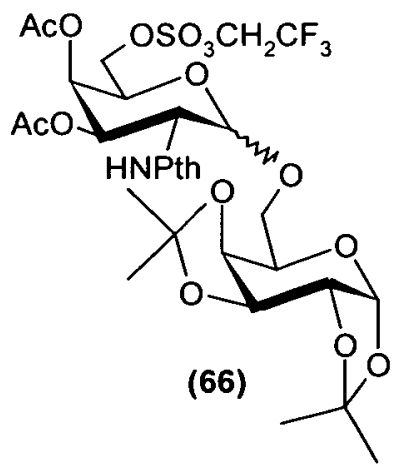

To a suspension of (55) (65mg, $0.1 \mathrm{mmol}), 1 ; 2,3 ; 4-d i-O$-isopropylidene- $\alpha-\mathrm{D}$ galactopyranose $(52 \mathrm{mg}, 0.2 \mathrm{mmol}), N$-iodosuccinamide $(25 \mathrm{mg}, 0.11 \mathrm{mmol})$ and powdered molecular sieves $(4 \AA)(\sim 100 \mathrm{mg})$ was added trifluoromethyl sulfonic acid $(5 \mu \mathrm{l})$. After $16 \mathrm{~h}$ only starting materials were visible on a TLC (7:3 diethyl ether: hexane) of the reaction. A sample of the reaction mixture was examined by Electrospray MS. peaks for (66) were visible $\mathrm{m} / \mathrm{z}$ (electrospray) $815\left(\mathrm{MNH}_{4}^{+}\right), 820$ $\left(\mathrm{MNa}^{+}\right)$. The solvent was removed from the reaction under reduced pressure and the resulting gum column chromatographed (7:3 diethyl ether : hexane). Fractions containing (66) by MS were combined and the solvent evaporated under reduced pressure however the resulting gum $(\sim 1 \mathrm{mg})$ could not be characterised.

Benzyl 2- $N$-acetamido-3- $O$-benzyl-2-deoxy-4;6- $O$-(4-methoxybenzylidene)- $\alpha$-Dglucopyranoside ${ }^{163}(69)$.

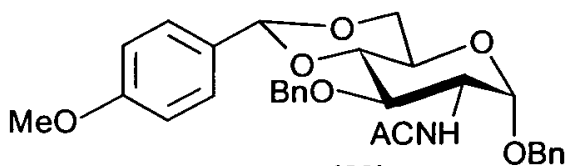

(69)

To a solution of $N$-acetyl glucosamine $(7.00 \mathrm{~g}, 31.6 \mathrm{mmol})$ in benzyl alcohol $\left(70 \mathrm{~cm}^{3}\right)$ at $95^{\circ} \mathrm{C}$ was added a sat. solution of hydrogen chloride gas in benzyl alcohol $\left(5 \mathrm{~cm}^{3}\right)$. After $20 \mathrm{~min}$ the mixture was filtered through a plug of glass wool into rapidly stirring diethyl ether $\left(300 \mathrm{~cm}^{3}\right)$. The resulting suspension was filtered and the solid 
washed repeatedly with diethyl ether $\left(4 \times 200 \mathrm{~cm}^{3}\right)$ and dried under vacuum to give (70) ${ }^{195}(8.55 \mathrm{~g}, 87 \%)$.

A solution of (70) $(8.55 \mathrm{~g}, 27.5 \mathrm{mmol}),(71)(14.84 \mathrm{~g}, 81.5 \mathrm{mmol})$ and para-toluene sulfonic acid $(0.01 \mathrm{~g}, 0.06 \mathrm{mmol})$ in $\operatorname{DMF}\left(50 \mathrm{~cm}^{3}\right)$ was heated at $70^{\circ} \mathrm{C}$ in an open topped vessel for $2 \mathrm{~h}$. The reaction was quenched by pouring the mixture into ice water $\left(200 \mathrm{~cm}^{3}\right)$ which had been made alkalis with two drops of sat. sodium bicarbonate soln.. The resulting suspension was filtered and the solid washed with water $\left(200 \mathrm{~cm}^{3}\right)$ and diethyl ether $\left(200 \mathrm{~cm}^{3}\right)$ then dried over $\mathrm{P}_{2} \mathrm{O}_{5}$ for $16 \mathrm{~h}$ in a vacuum desiccator to give $(\mathbf{7 2})^{196}(9.57 \mathrm{~g}, 81 \%)$ as a colourless solid.

To a suspension of (72) $(7.84 \mathrm{~g}, 18.3 \mathrm{mmol})$ and powdered sodium hydroxide $(3.79 \mathrm{~g}$, $94.7 \mathrm{mmol})$ in DMF $\left(100 \mathrm{~cm}^{3}\right)$ was added benzyl bromide $(16.23 \mathrm{~g}, 94.7 \mathrm{mmol})$. The mixture was stirred at room temperature for $16 \mathrm{~h}$. The solvent was then evaporated under reduced pressure and the resulting solid was recrystallised from ethanol to give (69) $(3.25 \mathrm{~g}, 35 \%)$ as a colourless solid; mp 239-241 ${ }^{\circ} \mathrm{C}$ (lit., $163239-245^{\circ} \mathrm{C}$ ); $v_{\max } / \mathrm{cm}^{-1}$ $3286,1651,1554,1209,821,733 ; \delta_{\mathrm{H}}\left(250 \mathrm{MHz} ; \mathrm{CDCl}_{3}\right) 1.78(3 \mathrm{H}, \mathrm{s}, \mathrm{NAc}), 3.60-$ 3.78 (4H, m, $\left.\mathrm{CH}_{2} \mathrm{Ph}\right), 3.72$ (3H, s, OMe), 4.08-4.28 (2H, m, 5, 6b-H), 4.33-4.64 (3H, $\mathrm{m}, 6 \mathrm{~b}, 4,2-\mathrm{H}), 4.76-4.87(2 \mathrm{H}, \mathrm{m}, 1,3-\mathrm{H}), 5.37-5.45$ (2H, m, $\left.\mathrm{PhCHO}_{2}, \mathrm{NH}\right), 6.80-$ $6.84\left(2 \mathrm{H}, \mathrm{m}\right.$, Arom.), 7.16-7.36 (12H, m, Arom.); $\delta_{\mathrm{C}}\left(62 \mathrm{MHz} ; \mathrm{CDCl}_{3}\right)$ 21.9, 51.0, $53.9,61.7,67.5,68.5,72.7,74.8,81.3,96.3,99.9,112.2,126.0,126.3,126.5,126.7$, $126.9,127.0,127.3,128.5,135.6,137.2,158.7,168.6 ; \mathrm{m} / \mathrm{z}$ (Electrospray) 520 $\left(\mathrm{MH}^{+}\right)$.

Benzyl 2-acetamido-3-O-benzyl-2-deoxy-6-O-(4-methoxybenzyl)- $\alpha$-Dglucopyranoside 163 (74) and Benzyl 2-acetamido-3-O-benzyl-2-deoxy-4-O-(4methoxybenzyl)- $\alpha$-D-glucopyranoside 163 (68).

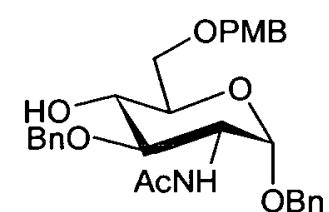

(74)

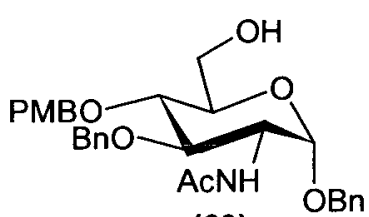

(68)

To a solution of (69) $(1.04 \mathrm{~g}, 2 \mathrm{mmol})$, sodium cyanoborohydride $(0.76 \mathrm{~g}, 12 \mathrm{mmol})$ and $3 \AA$ sieves $(100 \mathrm{mg})$ in dichloromethane $\left(50 \mathrm{~cm}^{3}\right)$ at $0^{\circ} \mathrm{C}$ was added a solution of 
trimethylsilyl chloride $(1.30 \mathrm{~g}, 12 \mathrm{mmol})$ in dichloromethane $\left(10 \mathrm{~cm}^{3}\right)$. The mixture was allowed to warm to room temperature. After $1 \mathrm{~h}$ the reaction was quenched by pouring the mixture into water $\left(50 \mathrm{~cm}^{3}\right)$. The product was extracted with ethyl acetate $(3 \times 75 \mathrm{ml})$ and the organic layers combined, washed with water $\left(50 \mathrm{~cm}^{3}\right)$, dried $\mathrm{MgSO}_{4}$ and evaporated under reduced pressure to give a colourless solid. The product was purified by column chromatography (1:1 ethyl acetate : ether) to give (74) $(0.12 \mathrm{~g}, 11.5 \%)$ as a colourless solid; $\mathrm{mp} 104-106^{\circ} \mathrm{C}$ (from ethyl acetate) (lit., 163 $\left.123-127^{\circ} \mathrm{C}\right) v_{\max } / \mathrm{cm}^{-1} 3297,2990,1646,1513,1208,1150,733 ; \delta_{\mathrm{H}}\left(250 \mathrm{MHz} ; \mathrm{CDCl}_{3}\right)$ $1.82(3 \mathrm{H}, \mathrm{s}, \mathrm{NAc}), 3.04(1 \mathrm{H}, \mathrm{bs}, 4-\mathrm{OH}), 3.66-3.77\left(6 \mathrm{H}, \mathrm{m}, \mathrm{CH}_{2} \mathrm{Ar}\right), 3.79(3 \mathrm{H}, \mathrm{m}$, OMe), 4.26 (1 H, ddd, J 10.0, J-NH $\left.9.0, \mathrm{~J}_{2-1} 3.5,2-\mathrm{H}\right), 4.40-4.82(5 \mathrm{H}, \mathrm{m}, 3,4,5,6 \mathrm{a}, 6 \mathrm{~b}-$ $\mathrm{H}), 4.88\left(1 \mathrm{H}, \mathrm{d}, \mathrm{J}_{1-2} 3.5,1-\mathrm{H}\right), 5.49\left(1 \mathrm{H}, \mathrm{d}, \mathrm{J}_{\mathrm{NH}-2} 9.0, \mathrm{NH}\right), 6.84-6.89$ (2H, m, Arom.), 7.20-7.39 (12H, m, Arom.); $\delta_{\mathrm{C}}\left(62 \mathrm{MHz} ; \mathrm{CDCl}_{3}\right) 23.1$ (NAc), $51.6(\mathrm{CH}), 55.0(\mathrm{OMe})$, $69.3\left(\mathrm{CH}_{2}\right), 69.6\left(\mathrm{CH}_{2}\right), 70.2(\mathrm{CH}), 71.9(\mathrm{CH}), 73.1\left(\mathrm{CH}_{2}\right), 73.6\left(\mathrm{CH}_{2}\right), 79.6(\mathrm{CH})$, 96.9 (1-C), 113.6, 127.5, 127.7, 127.8, 127.8, 128.1, 128.3, 128.3, 129.1, 129.2, $129.6,136.9,138.3,169.6(\mathrm{C}=\mathrm{O}) ; \mathrm{m} / \mathrm{z}$ (Electrospray) $522\left(\mathrm{MH}^{+}\right)$, and $(68)(83 \mathrm{mg}$, 8\%); mp 225-228 ${ }^{\circ} \mathrm{C}$ (decomposes) (lit., 163 185-189 ${ }^{\circ} \mathrm{C}$ ); $v_{\max } / \mathrm{cm}^{-1} 3290,1645,1514$, $1209,1066,821,638 ; \delta_{\mathrm{H}}\left(250 \mathrm{MHz} ; \mathrm{CDCl}_{3}\right) 1.79(3 \mathrm{H}, \mathrm{s}, \mathrm{NAc}), 3.69-3.79(6 \mathrm{H}, \mathrm{m}$, $\left.\mathrm{CH}_{2} \mathrm{Ar}\right), 3.79(3 \mathrm{H}, \mathrm{s}, \mathrm{OMe}), 4.17-4.90(7 \mathrm{H}, \mathrm{m}), 5.35(1 \mathrm{H}, \mathrm{d}, \mathrm{J} 9.5, \mathrm{NH}), 7.23-7.32$ (14H, m, Arom.); $\delta_{\mathrm{C}}\left(62 \mathrm{MHz} ; \mathrm{CDCl}_{3}\right) 23.2$ (NAc), $52.4(\mathrm{CH}), 55.1$ (OMe), 61.3 $\left(\mathrm{CH}_{2}\right), 69.6\left(\mathrm{CH}_{2}\right), 71.6(\mathrm{CH}), 74.7(\mathrm{CH}), 74.8\left(\mathrm{CH}_{2}\right), 77.6\left(\mathrm{CH}_{2}\right), 79.9(\mathrm{CH}), 97.0(1-$ C), $113.8,127.7,127.8,127.9,128.0,128.4,129.7,129.8,136.8,138.1,170.0$ $(\mathrm{C}=\mathrm{O}) ; \mathrm{m} / \mathrm{z}$ (Electrospray) $522\left(\mathrm{MH}^{+}\right)$. 
Allyl 2-acetamido-3,4-di- $O$-acetyl-2-deoxy- $\alpha$-D-glucopyranoside $\quad 6-\left(2^{\prime}, 2^{\prime}, 2^{\prime}-\right.$ trifluoroethyl sulfate) ${ }^{197}$ (76).

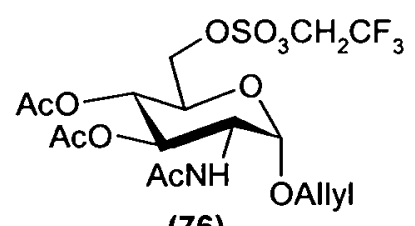

(76)

To a solution of allyl 2-acetamido-3,4-di- $O$-acetyl-2-deoxy-6- $O$-sulfo- $\alpha$-Dglucopyranoside trimethyl amine salt $197,198(702 \mathrm{mg}, 1.4 \mathrm{mmol})$ in acetonitrile was added (24) solution $(20 \mathrm{ml})$ and citric acid monohydrate $(\sim 1 \mathrm{~g})$. The mixture was stirred at room temperature for $16 \mathrm{~h}$ then the solvent was evaporated under reduced pressure and the resulting solid purified by column chromatography (1:3 hexane: ethyl acetate) to give (76) (386mg, 57\%) as a gummy solid; $v_{\max } / \mathrm{cm}^{-1} 3435,3029$, $1750,1679,1514,1424 ; \delta_{\mathrm{H}}\left(250 \mathrm{MHz} ; \mathrm{DMSO}^{-D_{6}}\right) 1.83(3 \mathrm{H}, \mathrm{s}, \mathrm{OAc}), 1.95(3 \mathrm{H}, \mathrm{s}$, OAc), 2.02 (3H, s, NAc), 4.07 (1H, m, 1'-H), $4.11(1 \mathrm{H}, \mathrm{m}, 5-\mathrm{H}), 4.21$ (1H, ddd, J $\left.11.0, \mathrm{~J}_{2-\mathrm{NH}} 9.0, \mathrm{~J}_{2-1} 3.5,2-\mathrm{H}\right), 4.22\left(1 \mathrm{H}, \mathrm{m}, \mathrm{l}^{\prime} \mathrm{H}\right), 4.51\left(2 \mathrm{H}, \mathrm{d}, \mathrm{J}_{6 \mathrm{a}, \mathrm{b}-\mathrm{5}} 3.5,6 \mathrm{a}, 6 \mathrm{~b}-\mathrm{H}\right), 4.89$ $\left(1 \mathrm{H}, \mathrm{d}, \mathrm{J}_{1-2} 3.5,1-\mathrm{H}\right), 4.96\left(1 \mathrm{H}, \mathrm{dd}, \mathrm{J}_{4-5} 10.5, \mathrm{~J}_{4-3} 9.0,4-\mathrm{H}\right), 5.10\left(2 \mathrm{H}, \mathrm{m}, \mathrm{CH}_{2} \mathrm{CF}_{3}\right) 5.20$ $\left(1 \mathrm{H}, \mathrm{dd}, \mathrm{J}_{3-2} 11.0, \mathrm{~J}_{3-4} 9.0,3-\mathrm{H}\right), 5.26\left(1 \mathrm{H}, \mathrm{m}, 3^{\prime}-\mathrm{H}\right), 5.40\left(1 \mathrm{H}, \mathrm{m}, 3^{\prime}-\mathrm{H}\right), 5.99(1 \mathrm{H}, \mathrm{m}$, 2'-H), $8.02\left(1 \mathrm{H}, \mathrm{d}, \mathrm{J}_{\mathrm{NH}-2}\right.$ 9.0, NH); $\delta_{\mathrm{C}}(62 \mathrm{MHz}$; DMSO-D 6 ) 20.5 (OAc), 20.5 (OAc), $22.3(\mathrm{NAc}), 50.3(\mathrm{CH}), 66.4(\mathrm{CH}), 66.6\left(\mathrm{q},{ }^{2} \mathrm{~J}_{\mathrm{C}-\mathrm{F}} 36.8, \mathrm{CH}_{2} \mathrm{CF}_{3}\right), 67.9(6-\mathrm{C}), 68.0$ (CH), 69.9 (CH), 72.1 (1'-C), 95.7 (1-C), 117.1 (3'-C), 122.4 (q, J J-F 277.6, CF $_{3}$ ), $133.6\left(2^{\prime}-\mathrm{C}\right), 169.6(\mathrm{C}=\mathrm{O}), 169.9(\mathrm{C}=\mathrm{O}), 169.9(\mathrm{C}=\mathrm{O}) ; \delta_{\mathrm{F}}\left(250 \mathrm{MHz} ; \mathrm{DMSO}_{6}\right)$ 74.8; m/z (FAB) $508\left(\mathrm{MH}^{+}\right)$(Found $\mathrm{MH}^{+}$, 508.1104. $\mathrm{C}_{17} \mathrm{H}_{25} \mathrm{~F}_{3} \mathrm{O}_{11} \mathrm{NS}$ requires $508.1100 \mathrm{dev} .4 \mathrm{ppm}$.$) .$ 

sulfate) $)^{197}(77)$.

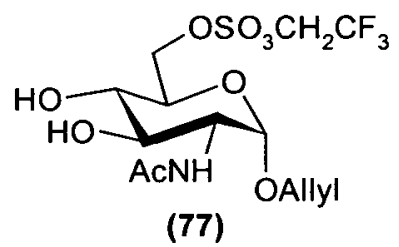

To a solution of $(76)(198 \mathrm{mg}, 0.4 \mathrm{mmol})$ in methanol $\left(20 \mathrm{~cm}^{3}\right)$ was added a solution of sodium methoxide $(63 \mathrm{mg}, 1.2 \mathrm{mmol})$ in methanol $\left(2 \mathrm{~cm}^{3}\right)$. After $15 \mathrm{~min}$ the reaction was quenched by the addition of Dowex $\left(\mathrm{H}^{+}\right)$ion exchange resin $(\sim 200 \mathrm{mg})$. The reaction was filtered and the filtrate was evaporated under reduced pressure to give (77) $(160 \mathrm{mg}, 97 \%)$ as a gum; $\delta_{\mathrm{H}}\left(250 \mathrm{MHz} ; \mathrm{DMSO}-\mathrm{D}_{6}\right) 1.83(3 \mathrm{H}, \mathrm{s}, \mathrm{NAc}), 3.24(1 \mathrm{H}$, m, 4-H), 3.56 (1H, m, 3-H), $3.74(1 \mathrm{H}, \mathrm{m}, 2-\mathrm{H}), 3.77(1 \mathrm{H}, \mathrm{m}, 5-\mathrm{H}), 3.98(1 \mathrm{H}, \mathrm{m}, 1$ 'H), $4.14\left(1 \mathrm{H}, \mathrm{m}, 1\right.$ '-H), $4.54\left(1 \mathrm{H}, \mathrm{dd}, \mathrm{J}_{6 \mathrm{a}-6 \mathrm{~b}} 10.5, \mathrm{~J}_{6 \mathrm{a}-5} 5.5,6 \mathrm{a}-\mathrm{H}\right), 4.59\left(1 \mathrm{H}, \mathrm{dd}, \mathrm{J}_{6 \mathrm{~b}-6 \mathrm{a}}\right.$ $\left.10.5, \mathrm{~J}_{5-6 \mathrm{~b}} 1.5,6 \mathrm{~b}-\mathrm{H}\right), 4.77\left(1 \mathrm{H}, \mathrm{d}, \mathrm{J}_{1-2} 3.5,1-\mathrm{H}\right), 5.03\left(1 \mathrm{H}, \mathrm{d}, \mathrm{J}_{\mathrm{OH}-3} 6.0,3-\mathrm{OH}\right), 5.10$ $\left(2 \mathrm{H}, \mathrm{m}, \mathrm{CH}_{2} \mathrm{CF}_{3}\right), 5.20\left(1 \mathrm{H}, \mathrm{m}, 3^{\prime}-\mathrm{H}\right), 5.35\left(1 \mathrm{H}, \mathrm{m}, 3^{\prime}-\mathrm{H}\right), 5.56\left(1 \mathrm{H}, \mathrm{d}, \mathrm{J}_{\mathrm{OH}-4} 6.0,4-\right.$ $\mathrm{OH}), 5.92\left(1 \mathrm{H}, \mathrm{m}, 2^{\prime}-\mathrm{H}\right), 7.85\left(1 \mathrm{H}, \mathrm{d}, \mathrm{J}_{\mathrm{NH}-2} 8.5, \mathrm{NH}\right) ; \delta_{\mathrm{C}}(62 \mathrm{MHz}$; DMSO-D $) 22.7$ (NAc), $53.3(\mathrm{CH}), 66.4$ (q, ${ }^{2} \mathrm{~J}_{\mathrm{C}-\mathrm{F}} 36.8, \mathrm{CH}_{2} \mathrm{CF}_{3}$ ), 67.2, (1'-C), $69.2(\mathrm{CH}), 69.9(\mathrm{CH})$, $70.1(\mathrm{CH}), 73.7$ (6-C), $95.8(\mathrm{CH}), 116.3$ (3'-C), 123.7 (q, J-F 277.4, CF 3 ), 133.9 (2'C), $169.8(\mathrm{C}=\mathrm{O}) ; \mathrm{m} / \mathrm{z}(\mathrm{FAB}) 424\left(\mathrm{MH}^{+}\right) ; \mathrm{C}_{13} \mathrm{H}_{21} \mathrm{~F}_{3} \mathrm{O}_{9} \mathrm{NS}$ requires 424.0889 found 424.0900 (dev. 11).

Phenyl 3,4,6-tri- $O$-acetyl-2-deoxy-2- $N$-pthalimido- $\alpha$-D-thiogalactopyranoside 199 (78).

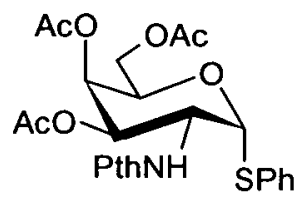

(78)

Phenyl 2-deoxy-2- $N$-pthalimido- $\alpha$-D-thiogalactopyranoside ${ }^{194}$ (62) (401 mg, $1 \mathrm{mmol}$ ) was added to a mixture of pyridine $\left(5 \mathrm{~cm}^{3}\right)$ and acetic anhydride $\left(5 \mathrm{~cm}^{3}\right)$ at $0^{\circ} \mathrm{C}$. The mixture was allowed to warm to room temperature over $2 \mathrm{~h}$. The solvent was then 
evaporated under reduced pressure and the resulting solid was purified by column chromatography (1:1 Ethyl acetate : hexane) to give (78) $(311 \mathrm{mg}, 60 \%)$ as a colourless solid; $\mathrm{mp} 143-146^{\circ} \mathrm{C}$ (from hexane) (lit., $199141-146^{\circ} \mathrm{C}$ ); $v_{\max } / \mathrm{cm}^{-1} 2916$, $2847,1744,1716,1471,1232,1072,719 ; \delta_{\mathrm{H}}\left(250 \mathrm{MHz} ; \mathrm{CDCl}_{3}\right) 1.83(3 \mathrm{H}, \mathrm{s}, \mathrm{OAc})$, $2.10(3 \mathrm{H}, \mathrm{s}, \mathrm{OAc}), 2.18(3 \mathrm{H}, \mathrm{s}, \mathrm{OAc}), 4.25-4.10(3 \mathrm{H}, \mathrm{m}, 5,6 \mathrm{a}, 6 \mathrm{~b}-\mathrm{H}), 4.59(1 \mathrm{H}, \mathrm{dd}$, $\left.\mathrm{J}_{2-3} 9.5, \mathrm{~J}_{2-1} 9.5,2-\mathrm{H}\right), 5.50\left(1 \mathrm{H}, \mathrm{d}, \mathrm{J}_{4-3} 3.5,4-\mathrm{H}\right), 5.75\left(1 \mathrm{H}, \mathrm{d}, \mathrm{J}_{1-2} 9.5, \mathrm{H}-1\right), 5.82(1 \mathrm{H}$, dd, $\mathrm{J}_{3-2}$ 9.5, $\mathrm{J}_{3-4}$ 3.5, 3-H), 7.24-7.30 (3H, m, Arom.), 7.35-7.50 (2H, m, Arom.), 7.71$7.79\left(2 \mathrm{H}, \mathrm{m}\right.$, Arom.), 7.83-7.88 (2H, m, Arom.); $\mathrm{m} / \mathrm{z}$ (Electrospray) $550\left(\mathrm{MNa}^{+}\right)$.

2- $N$-Acetyl-2-deoxy-4-(2,3,4,6-tetra- $O$-acetyl- $\beta$-D-glucopyranoside $\quad$ 6-(2',2',2'trifluoroethyl sulfate))- $\alpha$-D-glucopyranose (83).

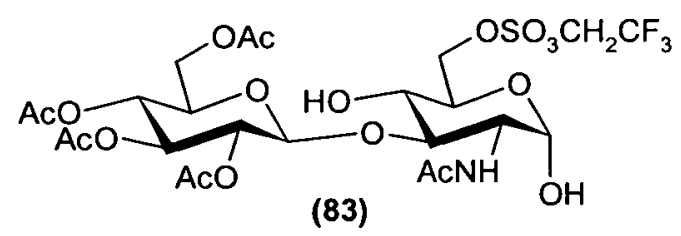

A suspension of (77) (160mg, $0.38 \mathrm{mmol}), 2,3,4,6$-tetra- $O$-acetyl- $\alpha$-D-glucopyranosyl bromide $(1.031 \mathrm{~g}, 2.47 \mathrm{mmol})$ and mercuric cyanide $(143 \mathrm{mg}, 0.57 \mathrm{mmol})$ in mixture of benzene $\left(5 \mathrm{~cm}^{3}\right)$ and nitromethane $\left(5 \mathrm{~cm}^{3}\right)$ was heated at $55^{\circ} \mathrm{C}$ for 6 days. Water $\left(10 \mathrm{~cm}^{3}\right)$ was added to the reaction and the product was extracted with diethyl ether (3 $\left.\mathrm{x} 10 \mathrm{~cm}^{3}\right)$. The combined organic layers were washed with brine $\left(10 \mathrm{~cm}^{3}\right)$ and the solvent was evaporated under reduced pressure to give a colourless gum that was purified by column chromatography ( $4: 1$ ethyl acetate : hexane) to give (83) $(60 \mathrm{mg}$, $21 \%)$ as a colourless solid; $[\alpha]_{\mathrm{D}}{ }^{16}+7.7^{\circ}\left(c 0.0275, \mathrm{CHCl}_{3}\right) ; \delta_{\mathrm{C}}\left(62 \mathrm{MHz} ; \mathrm{CDCl}_{3}\right) 14.3$ (NAc), 20.4 ( $2 \times \mathrm{OAc}), 20.5$ ( $2 \times \mathrm{OAc}), 61.1,65.4,66.4\left(\mathrm{q},{ }^{2} \mathrm{~J}_{\mathrm{C}-\mathrm{F}} 38.1, \mathrm{CH}_{2} \mathrm{CF}_{3}\right), 67.7$, $70.7,72.0,72.2,74.7,75.4,78.9,80.8,99.7(1-\mathrm{C}(\alpha)), 107.0\left(1^{\prime}-\mathrm{C}(\beta)\right), 123.7\left(\mathrm{q}, \mathrm{J}_{\mathrm{C}-\mathrm{F}}\right.$ 277.4, $\left.C \mathrm{~F}_{3}\right), 169.0(\mathrm{C}=\mathrm{O}), 169 .(\mathrm{C}=\mathrm{O}), 169.9(\mathrm{C}=\mathrm{O}), 170.4(\mathrm{C}=\mathrm{O}), 170.6(\mathrm{C}=\mathrm{O}) ; \delta_{\mathrm{F}}$ $\left(62 \mathrm{MHz} ; \mathrm{CDCl}_{3}\right)-74.08 ; \mathrm{m} / \mathrm{z}$ (Electrospray) $696(\mathrm{M}-\mathrm{OH}),(\mathrm{FAB}) \mathrm{C}_{24} \mathrm{H}_{35} \mathrm{~F}_{3} \mathrm{NO}_{18} \mathrm{~S}$ $\left(\mathrm{MH}^{+}\right)$requires 714.15270 , found 714.15385 (dev. 1.62).

\section{1;2,5;6-Di- $O$-isopropylidene-3-sulfo- $\alpha$-D-glucofuranose, sodium salt ${ }^{65}$ (84).}




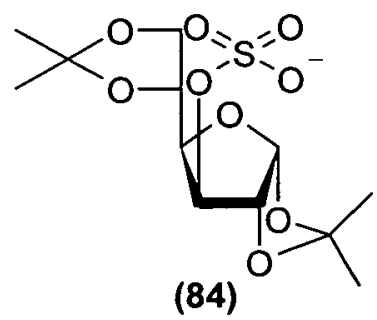

A solution of (20) $(50 \mathrm{mg}, 0.12 \mathrm{mmol})$ and sodium methoxide $(31 \mathrm{mg}, 0.6 \mathrm{mmol})$ in methanol $\left(5 \mathrm{~cm}^{3}\right)$ was stirred at room temperature for $16 \mathrm{~h}$. TLC (8:2 dichloromethane : methanol) shows no product. The mixture was then refluxed for $48 \mathrm{~h}$. The solvent was evaporated under reduced pressure and the resulting solid purified by column chromatography (3:1:0.01 dichloromethane : methanol :water) to give (84) $(44 \mathrm{mg}$, $99 \%)$ as a colourless solid; $v_{\max } / \mathrm{cm}^{-1} 3490,2940,1240,1000,840 ; \delta_{\mathrm{H}}(250 \mathrm{MHz}$; $\left.\mathrm{D}_{2} \mathrm{O}\right) 1.34\left(6 \mathrm{H}, \mathrm{s}, 2 \times \mathrm{CH}_{3}\right), 1.50\left(6 \mathrm{H}, \mathrm{s}, 2 \times \mathrm{CH}_{3}\right), 3.60\left(1 \mathrm{H}, \mathrm{dd}, \mathrm{J}_{6 \mathrm{~b}-6 \mathrm{a}} 12.1, \mathrm{~J}_{6 \mathrm{~b}-5} 6.0\right.$, $6 \mathrm{~b}-\mathrm{H}), 3.79\left(1 \mathrm{H}, \mathrm{dd}, \mathrm{J}_{6 \mathrm{a}-6 \mathrm{~b}} 12.0, \mathrm{~J}_{6 \mathrm{a}-5} 2.7,6 \mathrm{a}-\mathrm{H}\right), 3.90\left(1 \mathrm{H}, \mathrm{ddd}, \mathrm{J}_{5-4} 9.0, \mathrm{~J}_{5-6 \mathrm{~b}} 6.0, \mathrm{~J}_{5-6 \mathrm{a}}\right.$ 2.5, 5-H), $4.22\left(1 \mathrm{H}, \mathrm{dd}, \mathrm{J}_{4-5} 9.0, \mathrm{~J}_{4-3} 3.0,4-\mathrm{H}\right), 4.71\left(1 \mathrm{H}, \mathrm{d}, \mathrm{J}_{3-4} 3.0,3-\mathrm{H}\right), 4.86(1 \mathrm{H}, \mathrm{d}$, $\left.\mathrm{J}_{2-1} 4.0,2-\mathrm{H}\right), 6.03\left(1 \mathrm{H}, \mathrm{d}, \mathrm{J}_{1-2} 4.0,1-\mathrm{H}\right) ; \mathrm{m} / \mathrm{z}$ (-ve FAB) $339\left(\mathrm{M}^{-}\right)$.

\section{1;2,5;6-Di- $O$-isopropylidene-3-sulfo- $\alpha$-D-glucofuranose, potassium salt (84).}

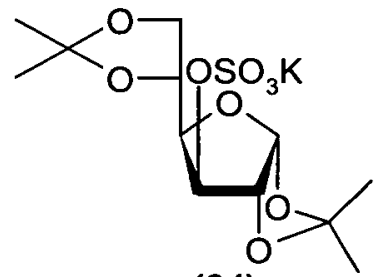

(84)

A solution of (20) $(100 \mathrm{mg}, 0.24 \mathrm{mmol})$ and potassium tert butoxide $(132 \mathrm{mg}$, $1.2 \mathrm{mmol})$ in tertbutanol $\left(10 \mathrm{~cm}^{3}\right)$ was heated at $80^{\circ} \mathrm{C}$ for $1.2 \mathrm{~h}$. The solvent was evaporated under reduced pressure and the resulting solid purified by column chromatography (4:2 dichloromethane : methanol) to give (84) $(73 \mathrm{mg}, 82 \%$ ) as a colourless solid; $\mathrm{mp} 157-159^{\circ} \mathrm{C}$ (from water) (lit., $65159-163^{\circ} \mathrm{C}$ ); $v_{\max } / \mathrm{cm}^{-1} 3490$, $2940,1240,1000,840 ; \delta_{\mathrm{H}}\left(250 \mathrm{MHz} ; \mathrm{D}_{2} \mathrm{O}\right) 1.34\left(6 \mathrm{H}, \mathrm{s}, 2 \times \mathrm{CH}_{3}\right), 1.50(6 \mathrm{H}, \mathrm{s}, 2 \mathrm{x}$ $\left.\mathrm{CH}_{3}\right), 3.60\left(1 \mathrm{H}, \mathrm{dd}, \mathrm{J}_{6 \mathrm{~b}-6 \mathrm{a}} 12.0, \mathrm{~J}_{6 \mathrm{~b}-5} 6.0,6 \mathrm{~b}-\mathrm{H}\right), 3.79\left(1 \mathrm{H}, \mathrm{dd}, \mathrm{J}_{6 \mathrm{a}-6 \mathrm{~b}} 12.0, \mathrm{~J}_{6 \mathrm{a}-5} 2.5,6 \mathrm{a}-\right.$ $\mathrm{H}), 3.90\left(1 \mathrm{H}, \mathrm{ddd}, \mathrm{J}_{5-4} 9.0, \mathrm{~J}_{5-6 \mathrm{~b}} 6.0, \mathrm{~J}_{5-6 \mathrm{a}} 2.5,5-\mathrm{H}\right), 4.22\left(1 \mathrm{H}, \mathrm{dd}, \mathrm{J}_{4-5} 9.0, \mathrm{~J}_{4-3} 3.0,4-\mathrm{H}\right)$, 
$4.71\left(1 \mathrm{H}, \mathrm{d}, \mathrm{J}_{3-4} 3.0,3-\mathrm{H}\right), 4.86\left(1 \mathrm{H}, \mathrm{d}, \mathrm{J}_{2-1} 3.5,2-\mathrm{H}\right), 6.03\left(1 \mathrm{H}, \mathrm{d}, \mathrm{J}_{1-2} 3.5,1-\mathrm{H}\right) ; \mathrm{m} / \mathrm{z}(-$ ve FAB) $339\left(\mathrm{M}^{-}\right)$.

1;2,3;4-Di- $O$-isopropylidene-6- $O$-sulfo-galactopyranose, sodium salt ${ }^{65}$

1;2,3;4-Di- $O$-isopropylidene-6- $O$-methyl -galactopyranose 200 (86).

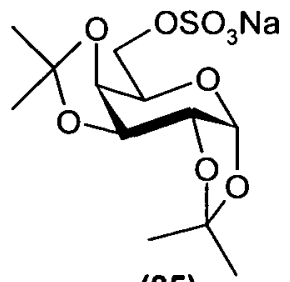

(85)

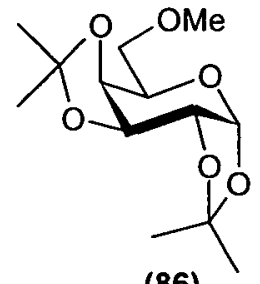

(86)

A solution of (30) $(50 \mathrm{mg}, 0.12 \mathrm{mmol})$ and sodium methoxide $(31 \mathrm{mg}, 0.6 \mathrm{mmol})$ in methanol was refluxed for $48 \mathrm{~h}$. The solvent was removed and the resulting solid was purified by column chromatography (dichloromethane then $7: 3$ dichloromethane : methanol) to give $(86)(17 \mathrm{mg}, 51 \%)$ as a colourless gum; $\delta_{\mathrm{H}}\left(250 \mathrm{MHz} ; \mathrm{CDCl}_{3}\right) 1.33$ $\left(3 \mathrm{H}, \mathrm{s}, \mathrm{CH}_{3}\right), 1.34\left(3 \mathrm{H}, \mathrm{s}, \mathrm{CH}_{3}\right), 1.46\left(3 \mathrm{H}, \mathrm{s}, \mathrm{CH}_{3}\right), 1.55\left(3 \mathrm{H}, \mathrm{s}, \mathrm{CH}_{3}\right), 3.39(3 \mathrm{H}, \mathrm{s}$, OMe), $3.54\left(1 \mathrm{H}, \mathrm{dd}, \mathrm{J}_{6 \mathrm{~b}-6 \mathrm{a}} 10.0, \mathrm{~J}_{6 \mathrm{~b}-5} 7.0,6 \mathrm{~b}-\mathrm{H}\right), 3.61\left(1 \mathrm{H}, \mathrm{dd}, \mathrm{J}_{6 \mathrm{a}-6 \mathrm{~b}} 10.0, \mathrm{~J}_{6 \mathrm{a}-5} 5.5,6 \mathrm{a}-\right.$ $\mathrm{H}), 3.97\left(1 \mathrm{H}, \mathrm{ddd}, \mathrm{J}_{5-6 \mathrm{~b}} 7.0, \mathrm{~J}_{5-6 \mathrm{a}} 5.5, \mathrm{~J}_{5-4} 2.0,5-\mathrm{H}\right), 4.24\left(1 \mathrm{H}, \mathrm{dd}, \mathrm{J}_{4-3} 7.5, \mathrm{~J}_{4-5} 2.0,4-\mathrm{H}\right)$, $4.31\left(1 \mathrm{H}, \mathrm{dd}, \mathrm{J}_{2-1} 5.0, \mathrm{~J}_{2-3} 2.5,2-\mathrm{H}\right), 4.60\left(1 \mathrm{H}, \mathrm{dd}, \mathrm{J}_{3-4} 7.5, \mathrm{~J}_{3-2} 2.5,3-\mathrm{H}\right), 5.54(1 \mathrm{H}, \mathrm{d}$, $\mathrm{J}_{1-2}$ 5.0, 1-H) (lit., 201 ; 5.54 (1-H), $4.25(2-\mathrm{H}), 4.56(3-\mathrm{H}), 4.42$ (4-H), 4.22 (5-H), 3.78 (OMe), $1.35\left(2 \times \mathrm{CH}_{3}\right), 1.45\left(\mathrm{CH}_{3}\right), 1.54\left(\mathrm{CH}_{3}\right)$. and (85) $(21 \mathrm{mg}, 49 \%)$ as a colourless solid; $v_{\max } / \mathrm{cm}^{-1} 3490,2940,1240,1000,840 ; \delta_{\mathrm{H}}\left(250 \mathrm{MHz} ; \mathrm{D}_{2} \mathrm{O}\right) 1.37(6 \mathrm{H}, \mathrm{s}, 2 \mathrm{x}$ $\left.\mathrm{CH}_{3}\right), 1.45\left(3 \mathrm{H}, \mathrm{s}, \mathrm{CH}_{3}\right), 1.54\left(3 \mathrm{H}, \mathrm{s}, \mathrm{CH}_{3}\right), 4.06\left(1 \mathrm{H}, \mathrm{dd}, \mathrm{J}_{6 \mathrm{~b}-6 \mathrm{a}} 11.5, \mathrm{~J}_{6 \mathrm{~b}-5} 9.0,6 \mathrm{~b}-\mathrm{H}\right)$, $4.19\left(1 \mathrm{H}, \mathrm{dd}, \mathrm{J}_{6 \mathrm{a}-6 \mathrm{~b}} 11.5, \mathrm{~J}_{6 \mathrm{a}-5} 4.0,6 \mathrm{a}-\mathrm{H}\right), 4.24\left(1 \mathrm{H}, \mathrm{ddd}, \mathrm{J}_{5-6 \mathrm{~b}} 9.0, \mathrm{~J}_{5-6 \mathrm{a}} 4.0, \mathrm{~J}_{5-4} 1.0,5-\mathrm{H}\right)$, $4.45\left(1 \mathrm{H}, \mathrm{dd}, \mathrm{J}_{4-3} 7.5, \mathrm{~J}_{4-5} 1.0,4-\mathrm{H}\right), 4.54\left(1 \mathrm{H}, \mathrm{dd}, \mathrm{J}_{2-1} 5.0, \mathrm{~J}_{2-3} 2.5,2-\mathrm{H}\right), 5.13(1 \mathrm{H}$, dd, $\left.\mathrm{J}_{3-4} 7.5, \mathrm{~J}_{3-2} 2.5,3-\mathrm{H}\right), 5.64\left(1 \mathrm{H}, \mathrm{d}, \mathrm{J}_{1-2} 5.0,1-\mathrm{H}\right) ; \delta_{\mathrm{C}}\left(62 \mathrm{MHz} ; \mathrm{D}_{2} \mathrm{O}\right) 22.2\left(\mathrm{CH}_{3}\right), 23.0$ $\left(\mathrm{CH}_{3}\right), 23.8\left(\mathrm{CH}_{3}\right), 23.8\left(\mathrm{CH}_{3}\right), 65.5(\mathrm{CH}), 65.9\left(\mathrm{CH}_{2}\right), 68.9(\mathrm{CH}), 69.0(\mathrm{CH}), 69.4$ (CH), $94.8(\mathrm{CH}), 109.1(\mathrm{C}), 109.3(\mathrm{C}) ; \mathrm{m} / \mathrm{z}(\mathrm{FAB}) 379\left(\mathrm{M}^{+}\right), \mathrm{C}_{12} \mathrm{H}_{20} \mathrm{KO}_{9} \mathrm{~S}$ requires 379.04652: found, 379.04618 (dev. -0.88). 


\section{Reaction of (30) and sodium hydride.}

Sodium hydride ( $75 \mathrm{mg}, 5.0 \mathrm{mmol}$, of a $60 \%$ suspension in mineral oil) was added to a solution of (30) $(150 \mathrm{mg}, 0.3 \mathrm{mmol})$ in THF $\left(5 \mathrm{~cm}^{3}\right)$. The mixture was stirred at room temperature for $16 \mathrm{~h}$. TLC shows no reaction.

\section{Reaction of (30) with potassium hydroxide under phase transfer conditions.}

A solution of $(30)(20 \mathrm{mg}, 0.05 \mathrm{mmol})$ in dichloromethane $\left(2 \mathrm{~cm}^{3}\right)$ was added to a solution of potassium hydroxide $(0.5 \mathrm{~g})$ and tetra-butyl ammonium bromide $(5 \mathrm{mg})$ in water $\left(5 \mathrm{~cm}^{3}\right)$. The mixture was heated to reflux for $24 \mathrm{~h}$. TLC (dichloromethane) indicated no reaction had taken place.

\section{Reaction of (20) and tributyltin ethoxide.}

A solution of (20) $(20 \mathrm{mg}, 0.05 \mathrm{mmol})$ and tributyltin ethoxide $(24 \mathrm{mg}, 0.075 \mathrm{mmol})$ in THF $\left(3 \mathrm{~cm}^{3}\right)$ was stirred at reflux for $48 \mathrm{~h}$. TLC (dichloromethane) indicated that no reaction had taken place.

\section{Reaction of (30) with 4-nitrophenyl benzaldoxime ${ }^{169}$.}

A mixture of (30) (50mg, 0.1 $\mathrm{mmol})$, 4-nitrobenzaldoxime (166 $\mathrm{mg}, 1.0 \mathrm{mmol})$, 1,1,3,3-tetra methyl guanidine $(115 \mathrm{mg}, 1.0 \mathrm{mmol})$ was dissolved in a mixture of 1,4dioxane $\left(5 \mathrm{~cm}^{3}\right)$ and water $\left(5 \mathrm{~cm}^{3}\right)$. The reaction was stirred at room temperature for $24 \mathrm{~h}$, no reaction was observed to have taken place by TLC.

\section{Reaction of (20) and potassium carbonate in acetonitrile.}

A suspension of (20) (5mg, 0.01 mmol), para-toluenesulfonic acid ( $5 \mathrm{mg}, 0.03 \mathrm{mmol})$ and potassium hydrogen carbonate $(25 \mathrm{mg}, 0.05 \mathrm{mmol})$ in acetonitrile $\left(1 \mathrm{~cm}^{3}\right)$ was stirred at room temperature for $2 \mathrm{~h}$. A new less polar product was observed that did not have the same $R_{F}$ value as (84). MS (electrospray) of the crude reaction mixture gave peaks for (20)- $\mathrm{C}_{3} \mathrm{H}_{4}\left(382+\mathrm{Na}^{+}\right)$i.e. one of the isopropylidene rings has been removed. 
Reaction of (30) and triethylamine in the presence of boron trifluoride.

A solution of (30) $(50 \mathrm{mg}, 0.1 \mathrm{mmol})$ and boron trifluoride etherate $\left(0.1 \mathrm{~cm}^{3}\right)$ in triethyl amine $\left(2 \mathrm{~cm}^{3}\right)$ was stirred at room temperature for $24 \mathrm{~h}$. TLC (dichloromethane) indicated no reaction had taken place.

\section{Deprotection of (30) by potassium tert-butoxide.}

A solution of (30) $(20 \mathrm{mg}, 0.05 \mathrm{mmol})$ and potassium tert-butoxide $(26 \mathrm{mg}, 0.25 \mathrm{mmol})$ in tert-butanol $\left(2 \mathrm{~cm}^{3}\right)$ was heated to $80^{\circ} \mathrm{C}$ for $1 \mathrm{~h}$. TLC (dichloromethane) indicated no starting material. TLC (10:1 dichloromethane : methanol) showed one spot that had the same $\mathrm{R}_{\mathrm{F}}$ as (85).

6-Sulfo-D-glucose, potassium salt ${ }^{202}(87)$.

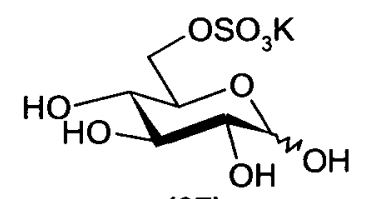

(87)

A solution of (29) $(51 \mathrm{mg}, 0.1 \mathrm{mmol})$ and potassium tert-butoxide (98mg, 1.0mmol) in tert-butanol was stirred at $80^{\circ} \mathrm{C}$ for $1 \mathrm{~h}$. The solution was then neutralised with dil. hydrochloric acid. The solvent was then evaporated under reduced pressure and the resulting solid purified by column chromatography (8:2 dichloromethane : methanol) to give the potassium salt of (87) (23mg, 88\%). $v_{\max } / \mathrm{cm}^{-1} 3440,2960,1243,1028$, $840 ; \quad \delta_{\mathrm{C}}\left(62 \mathrm{MHz} ; \mathrm{D}_{2} \mathrm{O}\right) \quad 67.82,70.69,70.80,72.19,73.58,93.20 ; \mathrm{MS}$ (-ve electrospray) $259 \mathrm{M}$.

Methyl 4;6-O-benzylidene-2-sulfo- $\alpha$-D-glucopyranoside, potassium salt (89).

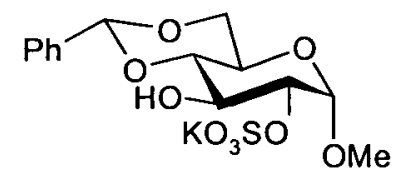

(89)

A mixture of (43) $(50 \mathrm{mg}, 0.1 \mathrm{mmol})$ and potassium tert-butoxide $(63 \mathrm{mg}, 0.5 \mathrm{mmol})$ in tert-butanol $\left(3 \mathrm{~cm}^{3}\right)$ was heated at $80^{\circ} \mathrm{C}$ for $1 \mathrm{~h}$. TLC (dichloromethane) indicated one new product that is less polar than the starting material. TLC ( $4: 1$ dichloromethane: 
methanol) indicated one new very polar product. The solution was neutralised with dil. hydrochloric acid and the solvent was evaporated under reduced pressure to give a yellow solid that was purified by column chromatography (4:1 dichloromethane : methanol) to give (89) $(40 \mathrm{mg}, 89 \%)$ as a colourless solid; $\delta_{\mathrm{H}}\left(250 \mathrm{MHz} ; \mathrm{CDCl}_{3}\right) 3.40$ (3H, s, OMe), 3.59 (1H, dd, J-3 $9.5, \mathrm{~J} 9.5,4-\mathrm{H}), 3.70\left(1 \mathrm{H}, \mathrm{dd}, \mathrm{J}_{6 \mathrm{~b}-6 \mathrm{a}} 10.0, \mathrm{~J} 1.0,6 \mathrm{~b}-\mathrm{H}\right)$, $3.79(1 \mathrm{H}, \mathrm{m}, 5-\mathrm{H}), 4.13\left(1 \mathrm{H}, \mathrm{dd}, \mathrm{J}_{3-4} 9.5, \mathrm{~J}_{3-2} 9.5,3-\mathrm{H}\right), 4.28\left(1 \mathrm{H}, \mathrm{dd}, \mathrm{J}_{6 \mathrm{a}-6 \mathrm{~b}} 10.0, \mathrm{~J} 4.5\right.$, 6a-H), $4.31(1 \mathrm{H}, \mathrm{bs}, 3-\mathrm{OH}), 4.43\left(1 \mathrm{H}, \mathrm{dd}, \mathrm{J}_{2-3} 9.5, \mathrm{~J}_{2-1} 4.0,2-\mathrm{H}\right), 5.00\left(1 \mathrm{H}, \mathrm{d}, \mathrm{J}_{1-2} 4.0\right.$, 1-H), 5.55 (1H, s, CHPh), 7.31-7.36 (3H, m, Arom.), 7.46-7.52 (2H, m, Arom.); $\delta_{\mathrm{C}}$ $\left(62 \mathrm{MHz} ; \mathrm{CDCl}_{3}\right) 55.2(\mathrm{OMe}), 62.5(\mathrm{CH}), 68.5(6-\mathrm{C}), 69.2(\mathrm{CH}), 78.2(\mathrm{CH}), 81.6$ (CH), $98.6(1-\mathrm{C}), 101.8(\mathrm{CHPh}), 126.3$ (2 x CH, Arom.), 127.6 (2 x CH, Arom.) 128.7 ( $\mathrm{CH}$, Arom.), 137.1 (C, Arom.).

1;2,3;4-Di- $O$-isopropylidene- $\alpha$-D-galactopyranose 6-(2',2'-difluoroethenyl sulfate) (91).

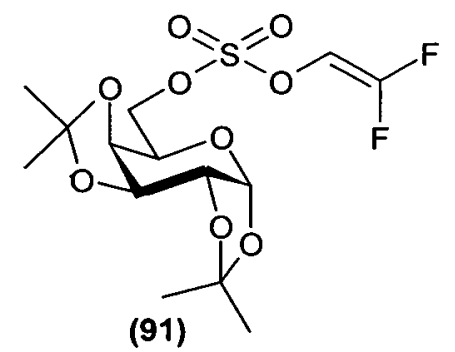

To a solution of (30) $(450 \mathrm{mg}, 1.06 \mathrm{mmol})$ in THF $\left(5 \mathrm{~cm}^{3}\right)$ at $-78^{\circ} \mathrm{C}$ was added $n$ butyllithium $(1.5 \mathrm{M}$ in hexanes) $(1.87 \mathrm{ml}, 2.65 \mathrm{mmol})$. The mixture was stirred at $78^{\circ} \mathrm{C}$ for $10 \mathrm{~min}$ before the reaction was quenched with methanolic ammonium chloride $\left(5 \mathrm{~cm}^{3}\right)$. The solvents were evaporated under reduced pressure and the resulting gum was dissolved into water $\left(10 \mathrm{~cm}^{3}\right)$ and extracted with diethyl ether $(3 \mathrm{x}$ $\left.10 \mathrm{~cm}^{3}\right)$. The combined organic layers were dried with $\mathrm{MgSO}_{4}$ and evaporated under reduced pressure to give a yellow gum that was purified by column chromatography (dichloromethane) to give (91) (342mg, 80\%) as a colourless gum; $v_{\max } / \mathrm{cm}^{-1} 2943$, $1457,1283,1009,840 ; \delta_{\mathrm{H}}\left(250 \mathrm{MHz} ; \mathrm{CDCl}_{3}\right) 1.26\left(3 \mathrm{H}, \mathrm{s}, \mathrm{CH}_{3}\right), 1.37\left(3 \mathrm{H}, \mathrm{s}, \mathrm{CH}_{3}\right)$, $1.45\left(3 \mathrm{H}, \mathrm{s}, \mathrm{CH}_{3}\right), 4.02-4.08(1 \mathrm{H}, \mathrm{m}, 5-\mathrm{H}), 4.16\left(1 \mathrm{H}, \mathrm{dd}, \mathrm{J}_{6 \mathrm{~b}-6 \mathrm{a}} 7.5, \mathrm{~J} 2.0,6 \mathrm{~b}-\mathrm{H}\right), 4.28$ $\left(1 \mathrm{H}, \mathrm{dd}, \mathrm{J}_{2-1} 5.0 \mathrm{~J} 2.5,2-\mathrm{H}\right), 4.34-4.15(2 \mathrm{H}, \mathrm{m}, 3,4-\mathrm{H}), 4.58\left(1 \mathrm{H}, \mathrm{dd}, \mathrm{J}_{6 \mathrm{a}-6 \mathrm{~b}} 7.5,6 \mathrm{a}-\mathrm{H}\right)$, 
$5.45\left(1 \mathrm{H}, \mathrm{d}, \mathrm{J}_{1-2} 5.0,1-\mathrm{H}\right), 6.29\left(1 \mathrm{H}, \mathrm{dd}, \mathrm{J}_{\mathrm{H}-\mathrm{F}} 13.9, \mathrm{~J}_{\mathrm{H}-\mathrm{F}} 4.0, \mathrm{C} H=\mathrm{CF}_{2}\right) ; \delta_{\mathrm{C}}(62 \mathrm{MHz}$; $\left.\mathrm{CDCl}_{3}\right) 23.3\left(\mathrm{CH}_{3}\right), 23.8\left(\mathrm{CH}_{3}\right), 24.8\left(2 \times \mathrm{CH}_{3}\right), 64.7,69.2,69.5,69.7,72.1,95.2$, $108.2,109.1 ; \delta_{\mathrm{F}}\left(250 \mathrm{MHz} ; \mathrm{CDCl}_{3}\right)-89.41\left(1 \mathrm{~F}, \mathrm{~d}, \mathrm{~J}_{\mathrm{F}-\mathrm{F}} 50.4\right)$, -109.90 (1F, d, J J 50.4$)$; $\mathrm{m} / \mathrm{z}$ (electrospray) $420\left(\mathrm{MNH}_{4}^{+}\right),(\mathrm{FAB}) \mathrm{C}_{14} \mathrm{H}_{21} \mathrm{~F}_{2} \mathrm{O}_{9} \mathrm{~S}\left(\mathrm{MH}^{+}\right)$requires 403.08744, found 403.08652 (dev. -2.27).

\section{1,2-Dibromo-2-methyl propane (93).}

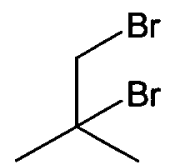

(93)

To a solution of potassium tert-butoxide $(1.12 \mathrm{~g}, 0.01 \mathrm{~mole})$, in tert-butanol was added (30) $(0.84 \mathrm{~g}, 0.002 \mathrm{~mole})$. The mixture was heated at $80^{\circ} \mathrm{C}$ for $1 \mathrm{hr}$. Nitrogen gas was passed across the reaction mixture and through a solution of bromine $(0.80 \mathrm{~g}$, $0.01 \mathrm{~mole})$ in dichloromethane $\left(100 \mathrm{~cm}^{3}\right)$ at $0^{\circ} \mathrm{C}$. After $1 \mathrm{hr}$ the bromine solution was added to aqueous sodium meta-bisulfite solution. The aqueous layer was extracted with dichloromethane $\left(3 \times 50 \mathrm{~cm}^{3}\right)$. The combined organic layers were dried $\left(\mathrm{MgSO}_{4}\right)$ and evaporated under reduced pressure to give (93) $(0.10 \mathrm{~g}, 23 \%)$ as a crude brown oil; $\delta_{\mathrm{H}}\left(250 \mathrm{MHz} ; \mathrm{CDCl}_{3}\right) 1.79\left(6 \mathrm{H}, \mathrm{s}, 2 \mathrm{x} \mathrm{CH}_{3}\right), 3.78\left(2 \mathrm{H}, \mathrm{s}, \mathrm{CBrH}_{2}\right)$.

\section{1;2,3;4-Di- $O$-isopropylidene- $\alpha$-D-galactopyranose $\quad$ 6-(2'-fluoro-2'-(tert-butoxy} ether)ethenyl sulfate) (99) and $1 ; 2,3 ; 4-D i-O$-isopropylidene- $\alpha$-Dgalactopyranose 6-(2',2'-difluoro-2'-(tert-butoxy ether)ethyl sulfate) (100).
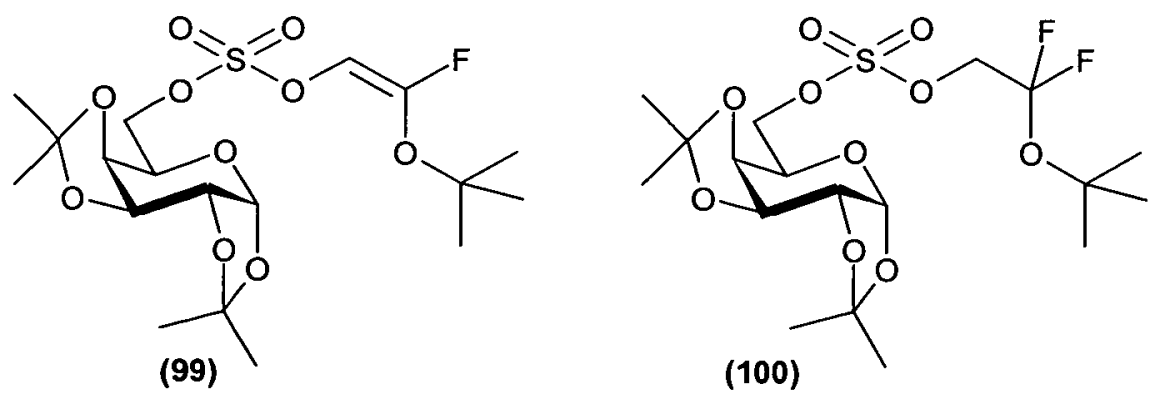

To a solution of potassium tert-butoxide $(140 \mathrm{mg}, 1.25 \mathrm{mmol})$ in tert-butanol $\left(5 \mathrm{~cm}^{3}\right)$ was added (91) $(50 \mathrm{mg}, 0.125 \mathrm{mmol})$ and the mixture stirred at room temperature for 
$30 \mathrm{~min}$ before the addition of ammonium chloride $(250 \mathrm{mg})$. The mixture was evaporated under reduced pressure to give a colourless solid that was dissolved in diethyl ether $\left(10 \mathrm{~cm}^{3}\right)$ and extracted with water $\left(5 \mathrm{~cm}^{3}\right)$. The organic layers were dried $\left(\mathrm{MgSO}_{4}\right)$ and evaporated under reduced pressure to give $(110 \mathrm{mg}, 80 \%)$ of (99) and $(\mathbf{1 0 0})$ as an inseparable mixture; $(\mathbf{9 9}) \delta_{\mathrm{H}}\left(250 \mathrm{MHz} ; \mathrm{CDCl}_{3}\right) 1.26\left(3 \mathrm{H}, \mathrm{s}, \mathrm{CH}_{3}\right), 1.36$ $\left(3 \mathrm{H}, \mathrm{s}, \mathrm{CH}_{3}\right), 1.37\left(6 \mathrm{H}, \mathrm{s}, 2 \mathrm{xCH}_{3}\right), 1.38\left(6 \mathrm{H}, \mathrm{s}, 2 \mathrm{xCH}_{3}\right), 1.46\left(3 \mathrm{H}, \mathrm{s}, \mathrm{CH}_{3}\right), 4.05-4.06$ $(1 \mathrm{H}, \mathrm{m}, 5-\mathrm{H}), 4.15-4.39(4 \mathrm{H}, \mathrm{m}), 4.57$ (1H, dd, J 7.5, J 2.5, 3-H), 5.46 (1H, d, J 5.0, $1-\mathrm{H}), 6.32\left(1 \mathrm{H}, \mathrm{d}, \mathrm{J}_{\mathrm{H}-\mathrm{F}} 4.0, \mathrm{HC}=\mathrm{CF}\right) ; \delta_{\mathrm{F}}\left(250 \mathrm{MHz} ; \mathrm{CDCl}_{3}\right)-99.67\left(\mathrm{~d}, \mathrm{~J}_{\mathrm{F}-\mathrm{H}} 3.8\right) ; \mathrm{m} / \mathrm{z}$ (Electrospray) $495\left(\mathrm{MK}^{+}\right):(\mathbf{1 0 0}) \delta_{\mathrm{H}}\left(250 \mathrm{MHz} ; \mathrm{CDCl}_{3}\right) 1.26\left(3 \mathrm{H}, \mathrm{s}, \mathrm{CH}_{3}\right), 1.36(3 \mathrm{H}, \mathrm{s}$, $\left.\mathrm{CH}_{3}\right), 1.37\left(6 \mathrm{H}, \mathrm{s}, 2 \mathrm{xCH}_{3}\right), 1.38\left(6 \mathrm{H}, \mathrm{s}, 2 \mathrm{xCH}_{3}\right), 1.46\left(3 \mathrm{H}, \mathrm{s}, \mathrm{CH}_{3}\right), 4.05-4.06(1 \mathrm{H}, \mathrm{m}$, 5-H), 4.15-4.39 (6H, m), $4.57(1 \mathrm{H}, \mathrm{dd}, \mathrm{J} 7.5, \mathrm{~J} 2.5,3-\mathrm{H}), 5.46(1 \mathrm{H}, \mathrm{d}, \mathrm{J} 5.0,1-\mathrm{H}), \delta_{\mathrm{F}}$ $\left(250 \mathrm{MHz} ; \mathrm{CDCl}_{3}\right)-73.23\left(\mathrm{dd}, \mathrm{J}_{\mathrm{F}-\mathrm{H}} 8.2, \mathrm{~J}_{\mathrm{Fa}-\mathrm{Fb}} 142.5\right) ; \mathrm{m} / \mathrm{z}$ (Electrospray) $515\left(\mathrm{MK}^{+}\right)$.

\section{6-Deoxy-1,2:3,4-di- $O$-isopropylidene-D-galacto-5-hexenoaldo-1,5-pyranose ${ }^{175}$} (96).

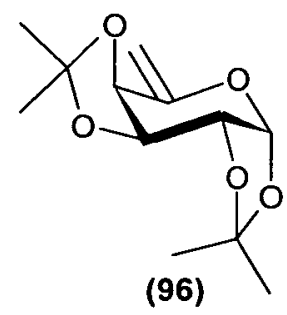

Potassium tert-butoxide (56mg, 0.5mmol) was added to a solution of (91) (40mg, $0.1 \mathrm{mmol})$ and diisopropyl carbodiimide $(19 \mathrm{mg}, 0.15 \mathrm{mmol})$ in hexane $\left(2 \mathrm{~cm}^{3}\right)$ and the mixture was heated at reflux for $2 \mathrm{hr}$. Water $\left(5 \mathrm{~cm}^{3}\right)$ was added and the mixture was extracted with diethyl ether $\left(3 \times 5 \mathrm{~cm}^{3}\right)$ the combined organic layers were dried $\left(\mathrm{MgSO}_{4}\right)$ and the solvent evaporated under reduced pressure. As the solvent evaporated crystals formed around the neck of the flask. The crystals were collected to give (96) $(18 \mathrm{mg}, 80 \%)$ as colourless needles; $\delta_{\mathrm{H}}\left(250 \mathrm{MHz} ; \mathrm{CDCl}_{3}\right) 1.34(3 \mathrm{H}, \mathrm{s}$, $\left.\mathrm{CH}_{3}\right), 1.36\left(3 \mathrm{H}, \mathrm{s}, \mathrm{CH}_{3}\right), 1.46\left(3 \mathrm{H}, \mathrm{s}, \mathrm{CH}_{3}\right), 1.47\left(3 \mathrm{H}, \mathrm{s}, \mathrm{CH}_{3}\right), 4.25(1 \mathrm{H}, \mathrm{dt}, \mathrm{J} 3.5, \mathrm{~J}$ 1.0, J 1.0), 4.57 (2H, d, J 1.0), 4.66 (1H, dd, J 1.0, J 1.0), 4.77 (1H, d, J 1.0), 5.05 $(1 \mathrm{H}, \mathrm{d}, \mathrm{J} 3.5), \delta_{\mathrm{C}}\left(62 \mathrm{MHz} ; \mathrm{CDCl}_{3}\right) 24.3,25.5,26.3,26.6\left(4 \times \mathrm{CH}_{3}\right), 70.6(\mathrm{CH}), 71.7$ 
Section Three: Experimental.

(CH), $72.8(\mathrm{CH}), 97.1(\mathrm{CH}), 100.4\left(\mathrm{CH}_{2}\right), 109.6(\mathrm{C}), 110.1(\mathrm{C}), 152.0$ (C) (lit., $\left.{ }^{175}\right)$; $\mathrm{m} / \mathrm{z}$ (Electrospray) $265\left(\mathrm{MNa}^{+}\right)$. 


\section{Section Four: Bibliography.}


1 G. O. Aspinall, 'Polysaccharides', Pergamon Press, 1970.

2 R. A. Dwek, C. J. Edge, J. D. Harvey, M. R. Wormald, R. B. Parekh, Annu. Rev. Biochem., 1993, 62, 65-100.

3 M. Metzler, A. Gertz, M. Sarkar, H. Schachter, J. W. Scharder, J. D. Marth, EMBO, 1994, 13, 2056-2065.

4 E. Ioffe, P. Stanley, Proc. Natl. Acad. Sci., 1994, 91, 728-732.

5 W. J. Lennarz, 'The biochemistry of glycoproteins and proteoglycans.', Plenum Press, 1980.

6 R. A. Kinloch, S. Mortillo, C. L. Stewart, P. M. Wassarman, J. Cell Biol., $1991,115,655-664$.

7 P. M. Wasserman, Development, 1990, 108, 1-17.

8 T. Feizi, M. Larkin, Glycobiology, 1991, 1, 17-24.

9 T. J. Matthews, K. J. Weinhold, H. K. Lyerly, A. J. Langlois, H. Wigzell, D. P. Bolognesi, Proc. Natl. Acad. Sci. USA., 1987, 84, 5424-5428.

10 B. D. Walker, M. Kowalski, W. C. Goh, K. Kozarsky, M. Krieger, C. Rosen, L. Rohrschneider, W. A. Hasltine, J. Sodroski, Proc. Natl. Acad. Sci. USA., 1987, 84, 8120-8124.

11 I. Ofkel, N. Sharon, Curr. Top. Microbiol. Immunol., 1990, 151, 91-114.

12 K.-A. Karlsson, Annu. Rev. Biochem., 1989, 58, 309-350.

13 E. Bremer, S. Hakomori, D. Bowen-Pope, E. Raines, R. Ross, J. Biol. Chem., $1984,259,6818-6825$.

14 M. A. Recny, M. A. Luther, M. H. Knoppers, E. A. Neidhardt, S. S. Khandekar, M. F. Concino, P. A. Schimke, M. A. Franics, U. Moebius, B. B. Reinhold, V. N. Reinhold, E. L. Reinhertz, J. Biol. Chem., 1992, 267, 22428-22434.

15 T. Feizi, Trends Biochem. Sci., 1991, 16, 84-86.

16 J. C. McAuliffe, O. Hindsgaul, Chem. Ind. (London), 1997, 170-174.

17 A. Varki, Glycobiology, 1993, 3, 97-130. 
18 L. V. Hooper, S. M. Manzella, J. U. Baenziger, FASEB J., 1996, 10, $1137-$ 1146.

19 E. G. V. Percival, Quart. Rev., 1949, 3, 369-384.

20 H. P. Rang, M. M. Dale, in 'Pharmocology', London, 1991.

21 R. Linhardt, Chem. Ind. (London), 1991, 45-50.

22 P. D. J. Grootenhuis, P. Westerduin, D. Meuleman, M. Petitou, C. A. A. v. Boeckel, Nature: Structural Biology, 1995, 2, 736-739.

23 L. Thunberg, G. Backstrom, U. Lindahl, Carbohydr. Res., 1982, 100, 393410.

24 U. Lindahl, 'Heparin: chemical and biological properties, clinical applications.', ed. D. A. Lane and U. Lindahl, CRC Press Inc., 1989.

25 O. Saksela, D. Moscatelli, A. Sommer, D. B. Rifkin, J. Cell Biol., 1988, 107, 743-751.

26 J. E. Turnbull, D. G. Fernig, Y. Ke, M. C. Wilkinson, J. T. Gallagher, J. Biol. Chem., 1992, 267, 10337-10341.

27 D. A. Kessler, R. S. Langer, N. A. Pless, J. Folkman, Int. J. Cancer, 1976, 18, 703-709.

28 A. W. Clowes, M. J. Karnovsky, Nature, 1977, 265, 625-626.

29 M. Maccarana, B. Casu, U. Lindahl, J. Biol. Chem., 1993, 268, 23898-23905.

30 J. Sudhalter, J. Flokman, C. M. Svahn, K. Bergendal, P. A. D'Amore, J. Biol. Chem., 1989, 264, 6892-6897.

31 J. Westman, M. Nilsson, D. M. Ornitz, C. M. Svahn, J. Carbohydr. Chem., 1995, 14, 95-113.

32 J. Westman, M. Nilsson, J. Carbohydr. Res., 1995, 14, 949-960.

33 M. Witvrouw, E. DeClercq, General Pharmacology, 1997, 29, 497-511.

34 M. Baba, Proc. Natl. Acad. Sci. USA, 1988, 85, 6132-6136. 
35 P. Lerouge, P. Roche, C. Faucher, F. Maillet, G. Truchet, J.-C. Prome, J. Denarie, Nature, 1990, 344, 781.

36 S. Long, The Plant Cell, 1996, 8, 1885-1898.

37 W. J. Sanders, T. R. Katsumoto, C. R. Bertozzi, S. D. Rosen, L. L. Kiessling, Biochemistry, 1996, 35, 14862-14867.

38 J. Whelan, Trends Biochem. Sci., 1996, 21, 65.

39 W. Chai, M. Larkin, M. S. Stoll, A. C. Stuart, F. X. Sullivan, T. J. Ahern, T. Feizi, Biochemistry, 1992, 31, 9126-9131.

40 C. T. Yuen, K. Bezouska, J. O'Brien, M. Stoll, R. Lemoine, A. Lubineau, M. Kiso, A. Hasegawa, N. J. Bockovich, K. C. Nicolaou, T. Feizi, J. Biol. Chem., 1994, 269, 1595-1598.

41 R. Sawada, S. Tsuboi, M. Fukuda, J. Biol. Chem., 1994, 269, 1425-1431.

42 A. E. Koch, M. M. Halloran, C. J. Haskell, M. R. Shah, P. J. Polverini, Nature, 1995, 376, 517-519.

43 L. L. Kiessling, N. L. Pohl, Chemistry \& Biology, 1996, 3, 71-77.

44 L. L. Kiessling, E. J. Gordon, Chemistry \& Biology, 1998, 5, R49-R62.

45 E. J. Gordon, W. J. Sanders, L. L. Kiessling, Nature, 1998, 392, 30-31.

46 N. M. Hooper, E. H. Karran, A. J. Turner, Biochem. J., 1997, 321, 265-279.

47 W. B. Huttner, Trends Biochem. Sci., 1987, 361-363.

48 W. B. Huttner, P. A. Baeuerie, in 'Protein Sulfation on Tyrosine.', ed. B. Satir, 1987.

49 P. P. Wilkins, K. L. Moore, R. P. McEver, R. D. Cummings, J. Biol. Chem., 1995, 270, 22677-22680.

50 J. M. Kornprobst, C. Sallenave, G. Barnathan, Comp. Biochem. Physiol. B., $1998,119,1-51$.

51 K. Ishida, M. Murakami, H. Matsuda, K. Yamaguchi, Tetrahedron Lett., $1995,36,3535-3538$. 
52 B. W. Sullivan, D. J. Faulkner, G. K. Matsumoto, H. Cun-Heng, J. Clardy, J. Org. Chem., 1986, 51, 4568-4573.

53 E. Finamore, F. Zolla, L. Minale, T. Yasumoto, J. Nat. Prod., 1992, 55, 767772.

54 M. Murata, T. Iwashita, A. Yokohama, M. Sasaki, T. Yasumota, J. Am. Chem. Soc., 1992, 114, 6594-6596.

55 M. Murata, H. Naoki, T. Iwashita, S. Matsunaga, M. Sasaki, A. Yokohama, T. Yasumoto, J. Am. Chem. Soc., 1993, 115, 2060-2062.

56 P. W. Robbins, F. Lippman, J. Am. Chem. Soc., 1956, 78, 2652-2654.

57 W. B. Jakoby, R. D. Sekura, E. S. Lyon, C. J. Marcus, J.-L. Wang, in 'Sulfotransferases', ed. W. B. Jakoby, New York, 1980.

58 E. Baumman, Ber. Dtsch. Chem. Ges., 1876, 54.

59 C. N. Falany, FASEB J., 1997, 11, 206-216.

60 W. B. Huttner, Annu. Rev. Physiol., 1988, 50, 363-376.

61 J. A. Roth, Trends Pharmacol. Sci., 1986, 7, 404-407.

62 C.-H. Lin, G.-J. Shen, E. Garcia-Junceda, C.-H. Wong, J. Am. Chem. Soc., $1995,29,8031-8032$.

63 E. G. V. Percival, J. Chem. Soc., 1945, 119-123.

64 D. L. Ingles, Chem. Ind. (London), 1960, 1159.

65 R. O. Mumma, C. P. Hoiberg, R. Simpson, Carbohydr. Res., 1970, 14, $119-$ 122.

66 C. P. Hoiberg, R. O. Mumma, Biochem. Biophys. Acta., 1969, 177, 149.

67 C. P. Hoiberg, R. O. Mumma, J. Am. Chem. Soc., 1969, 91, 4273.

68 T. J. Tewson, J. Org. Chem., 1983, 48, 3507-3510.

69 H. K. Garner, H. J. Lucas, J. Am. Chem. Soc., 1950, 72, 5497-5501.

70 M. S. Berridge, M. P. Franceschini, E. Rosenfeld, T. J. Tewson, J. Org. Chem., 1990, 55, 1211-1217. 
71 Y. Gao, K. B. Sharpless, J. Am. Chem. Soc., 1988, 110, 7538-7539.

72 A. B. Foster, E. B. Hancock, J. Chem. Soc., 1957, 968-970.

73 J. Attenburrow, A. F. B. Cameron, J. H. Chapman, R. M. Evans, B. A. Hems, A. B. A. Jansen, T. Walker, J. Chem. Soc., 1952, 1094-1111.

74 K. C. Nicolaou, N. J. Bockovich, D. R. Carcanague, C. W. Hummel, L. F. Even, J. Am. Chem. Soc., 1992, 114, 8701-8702.

75 I. Robina, E. Lopez-Barba, J. Jimenez-Barbero, M. Martin-Pastor, J. Fuentes, Tetrahedron: Asymmetry, 1997, 8, 1207-1224.

76 S. Ikeshita, A. Sakamoto, Y. Nakahara, Y. Nakahara, T. Ogawa, Tetrahedron Lett., 1994, 35, 3123-3126.

77 L. X. Wang, C. Li, Q. Wang, Y. Hui, Tetrahedron Lett., 1993, 34, 7763-7766.

78 D. Tailler, J. C. Jacquinet, J. M. Beau, J. Chem. Soc., Chem. Commun., 1994, 1827-1828.

79 L. Wang, C. Li, Q. Wang, Y. Hui, J. Chem. Soc., Perkin Trans 1, 1994, 621628.

80 E. E. Gilbert, Chem. Rev., 1962, 62, 549-589.

81 L. Cipolla, L. Lay, F. Nicotra, L. Panza, G. Russo, Tetrahedron Lett., 1994, 35, 8669-8670.

82 J. R. Turvey, Adv. Carbohydr. Chem., 1965, 20, 183-218.

83 Y. Tsuda, M. Nishimura, T. Kobayashi, Y. Sato, K. Kanemitsu, Chem. Pharm. Bull., 1991, 39, 2883-2887.

84 S. David, S. Hanessian, Tetrahedron, 1985, 41, 643-663.

85 M. A. Nashed, L. Anderson, Tetrahedron Lett., 1976, 39, 3503-3506.

86 S. Kopper, A. Brandenburg, Liebigs. Ann. Chem., 1992, 933-940.

87 R. M. Munavu, H. Szmant, J. Org. Chem., 1976, 41, 1832-1835.

88 S. David, C. Pascard, M. Cesario, Nouv. J. Chimie, 1979, 3, 63-68. 
89 A. Glen, D. A. Leigh, R. P. Martin, J. P. Smart, A. M. Truscello, Carbohydr. Chem., 1993, 248, 365-369.

90 D. A. Leigh, R. P. Martin, J. P. Smart, A. M. Truscello, J. Chem. Soc. Chem. Commun., 1994, 1373-1374.

91 B. Guilbert, N. J. Davis, S. L. Flitsch, Tetrahedron Lett., 1994, 35, 65636566.

92 B. Guilbert, N. J. Davis, M. P. Pearce, R. T. Aplin, S. L. Flitsch, Tetrahedron: Asymmetry, 1994, 5, 2163-2178.

93 S. Langston, B. Bernet, A. Vasella, Helv. Chim. Acta., 1994, 77, 2341-2353.

94 A. Lubineau, R. Lemoine, Tetrahedron Lett., 1994, 35, 8795-8796.

95 S. Cofefey, in 'Rodd's chemistry of carbon compounds', New York, 1967.

96 J. R. Turvey, T. P. Williams, J. Chem. Soc., 1962, 21 19-2122.

97 E. T. Kaiser, in 'Reactions of Sulfonate and Sulfate Esters', ed. S. Oae, New York and London, 1944.

98 C. Barkenbus, J. J. Owen, J. Am. Chem. Soc., 1934, 56, 1204-1206.

99 C. M. Sutter, 'The Organic Chemistry of Sulfur', John Wiley and Sons, 1944.

100 J. Mckenna, J. K. Norymberski, J. Chem. Soc., 1957, 3893-3900.

101 R. E. Robertson, S. E. Sugamori, Can. J. Chem., 1966, 44, 1728.

102 A. B. Foster, E. B. Hancock, Overend, Rob, J. Chem. Soc., 1956, 2589.

103 K. K. Ogilvie, S. L. Beaucage, Chem. Commun., 1976, 443-444.

104 M. M. Abel-Malik, A. S. Perlin, Carbohydr. Res., 1989, 190, 39-52.

105 R. B. Woodward, K. Heusler, J. Gosteli, P. Nageli, W. Oppolzer, R. Ramage, S. Ranganathan, H. Vorbruggen, J. Am. Chem. Soc., 1966, 88, 852-853.

106 O. Paleta, A. Danda, L. Stepan, J. Kvicala, V. Dedek, J. Fluorine Chem., $1989,45,331-348$.

107 C. L. Penney, A. S. Perlin, Carbohydr. Res., 1981, 93, 241-246.

108 J. Charalambous, M. J. Frazer, W. Gerrad, J. Chem. Soc., 1964, 5480-5482. 
109 D. R. Bundle, J. Chem. Soc., PerkinTrans. 1, 1979, 2751-2755.

110 M. G. Ambrose, R. W. Binkley, J. Org. Chem., 1983, 48, 674-677.

111 H. J. Jennings, J. K. N. Jones, Can. J. Chem., 1965, 43, 2372-2386.

112 S. Hanessian, J. M. Vatele, Tetrahedron Lett., 1981, 22, 3579-3582.

113 M. S. Berridge, M. P. Franceschini, E. Rosenfeld, T. J. Tewson, J. Org. Chem., 1990, 55, 1211-1217.

114 Y. Wiesel, Y. Liwschitz, Org. Prep. Proceed. Int., 1972, 4, 79-81.

115 M. Hedayatullah, J. C. Leveque, L. Denivelle, C. R. Seances Acad. Sci. Serie C., 1972, 274, 1937-1940.

116 C. O. Meese, Synthesis, 1984, 12, 1041-1042.

117 H. Gilman, R. G. Jones, J. Am. Chem. Soc., 1943, 65, 1458-1460.

118 F. Jin, Y. Xu, Y. Ma, Tetrahedron Lett., 1992, 33, 6161-6164.

119 W. R. Bamford, T. S. Stevens, J. Chem. Soc., 1952, 4735-4740.

120 H. Gilman, D. J. Peterson, D. Wittenberg, Chem. \& Ind., 1958, 1479-1480.

121 F. Jin, Y. Xu, B. Jiang, Tetrahedron Lett., 1992, 33, 1221-1224.

122 H. D. Martin, R. Iden, F. J. Mais, G. Kleefeld, A. Steigel, B. Fuhr, O. Rummele, A. Oftring, E. Schwichtenberg, Tetrahedron Lett., 1983, 24, 5469-5472.

123 A. R. Bassindale, A. G. Brook, Can. J. Chem., 1974, 52, 3474-3483.

124 J. Ho, J. C. Fishbein, J. Am. Chem. Soc., 1994, 116, 6611-6621.

125 A. V. Manuilov, V. A. Barkhash, Russ. Chem. Rev. (Engl. Transl.), 1990, 59, 179-192.

126 B. Helferich, J. Zirner, Chem. Ber., 1962, 95, 2604-2611.

127 P. Collins, R. Ferrier, 'Monosaccharides. Their chemistry and their roles in natural products.', John Wiley \& Sons Ltd., 1996.

128 O. Varela, D. Cicero, R. M. d. Lederkremer, J. Org. Chem., 1989, 54, 18841890.

129 Neilson, , ed. Patai, New York, 1975. 
130 W. A. Szarek, A. Zamojski, K. N. Tiwari, E. R. Isoni, Tetrahedron Lett., $1986,27,3827-3830$.

131 A. N. d. Belder, Adv. Carbohydr. Chem., 1965, 20, 219.

132 K. S. Kim, Y. H. song, B. H. Lee, C. S. Hahn, J. Org. Chem., 1986, 51, 404407.

133 S. J. Angyal, R. J. Beveridge, Carbohydr. Res., 1978, 65, 229-234.

134 A. D. Proud, J. C. Prodger, S. L. Flitsch, Tetrahedron Lett., 1997, 38, 7243 7246.

135 K. Toshima, K. Tatsuta, Chem. Rev., 1993, 93, 1503-1531.

136 G. J. Boons, P. Grice, R. Leslie, S. V. Ley, L. L. Yeung, Tetrahedron Lett., $1993,34,8523-8526$.

137 D. Kahne, S. Walker, Y. Cheng, D. V. Engen, J. Am. Chem. Soc., 1989, 111, 6881-6882.

138 K. Takeo, K. Maki, Y. Wada, S. Kitamura, Carbohydr. Res., 1993, 245, 8196.

139 T. Shimadate, S. Chiba, K. Inouye, T. Iino, Y. Hosoyama, Bull. Chem. Soc. Jpn., 1982, 55, 3552-3554.

140 S. Nambiar, J. F. Daeuble, R. J. Doyle, K. G. Taylor, Tetrahedron Lett., 1989, 30, 2179-2182.

141 T. Kametani, K. Kawamura, T. Honda, J. Am. Chem. Soc., 1986, 109, 30103017.

142 F. Dasgupta, P. J. Garegg, Acta. Chem. Scand., 1989, 43, 471-475.

143 W. Koenigs, E. Knorr, Ber., 1901, 34, 957-981.

144 M. Kloosterman, M. J. Dees, G. A. v. d. Marel, J. H. Boom, Recl. Trav. Chim. Pays-Bas, 1985, 104, 116-119.

145 R. R. Schmidt, W. Kinzy, Adv. Carbohydr. Chem. Biochem., 1994, 50, $21-$ 123. 
146 T. Itoh, H. Takamura, K. Watanabe, Y. Araki, Y. Ishido, Carbohydr. Res., $1986,156,241-246$.

147 R. R. Schmidt, J. Michel, M. Roos, Liebigs Ann. Chem., 1984, 1343-1357.

148 D. Lafont, P. Boullanger, O. Cadas, G. Descotes, Synthesis, 1989, 191-194.

149 P. M. Collins, W. G. Overend, B. A. Rayner, Carbohydr. Res., 1973, 39, 116.

150 K. Saito, K. Ichinose, Y. Sasaki, S. Sumita, Chem. Pharm. Bull., 1992, 40, 3261-3268.

151 W. J. Hickinbottom, J. Chem. Soc., 1929, 1676-1686.

152 M. Bols, Acta. Chem. Scand., 1993, 47, 829-834.

153 A. G. Brown, D. F. Corbett, A. J. Eglington, T. T. Howarth, Tetrahedron, $1983,39,2551-2562$.

154 K. A. Potekhin, A. V. Maleev, Y. T. Struchkov, A. M. Magerramov, A. S. Kozmin, N. S. Zefirov, Dokl. Akad. Nauk. SSSR., 1990, 311, 1390-1393.

155 N. S. Zefirov, N. V. Zyk, Y. A. Lapin, A. G. Kutateladze, V. N. Panov, A. V. Goncharov, E. N. Kurkutova, Y. T. Struchkov, Dokl. Acad. Nauk. SSSR., 1989, 307, 378-382.

156 K. C. Nicolaou, S. P. Seitz, D. P. Papahatjis, J. Am. Chem. Soc., 1983, 105, 2430-2434.

157 W. R. Roush, X. Lin, J. A. Straub, J. Org. Chem., 1991, 56, 1649-1655.

158 M. Sasaki, K. Tachibana, Tetrahedron Lett., 1991, 32, 6873-6876.

159 D. Kahne, S. Walker, Y. Cheng, D. V. Engen, J. Am. Chem. Soc., 1986, 111, 6881-6882.

160 D. Crich, S. Sun, J. Org. Chem., 1997, 62, 1198-1199.

161 P. Brigl, Z. Physiol. Chem., 1921, 116, 1.

162 D. A. Rosenfeld, N. K. Richtmyer, C. S. Hudson, J. Am. Chem. Soc., 1948, 70, 2201-2206. 
163 R. Johansson, B. Samuelsson, J. Chem. Soc., Perkin Trans. 1, 1984, 23712374.

164 G. Wulff, G. Rohle, Angew. Chem., Int. Ed. Engl., 1974, 13, 157-216.

165 P. J. Garegg, H. Hutberg, S. Wallin, Carbohydr. Res., 1982, 108, 97-101.

166 A. Liptak, I. Jodal, P. Nansi, Carbohydr. Res., 1975, 44, 1.

167 R. Baumann, Unpublished Work, 1997.

168 R. Vig, R. K. Jain, C. F. Piskoz, K. L. Matta, J. Chem. Soc., Chem. Commun, 1995, 2073-2074.

169 C. B. Reese, R. C. Titmas, L. Yau, Tetrahedron Lett., 1978, 30, 2727-2730.

170 J. Ichikawa, T. Moriya, T. Sonada, H. Kobayashi, Chem. Lett., 1991, 961964.

171 H. R. Allcock, P. R. Suszko, T. L. Evans, Organometalics, 1982, 1, 14431449 .

172 D. Gupta, R. Soman, S. Dev, Tetrahedron, 1982, 38, 3013-3018.

173 J. Mulzer, B. Schollhorn, Angew. Chem., Int. Ed. Engl., 1990, 29, 431-432.

174 F. C. Chang, Tetrahedron Lett., 1964, 6, 305-309.

175 F. Sarabia-Garcia, F. J. Lopez-Herrera, M. S. P. Gonzalez, Tetrahedron, 1985, 51, 5491-5500.

176 O. P. Strausz, R. J. Norstrom, Theoret. Chim. Acta (Berl.), 1973, 29, 183-187.

177 O. P. Strausz, R. J. Norstrom, D. Salahub, R. K. Gosavi, H. E. Gunning, I. G. Csizadia, J. Am. Chem. Soc., 1970, 92, 6395-6402.

178 R. J. Norstrom, H. E. Gunning, O. P. Srtausz, J. Am. Chem. Soc., 1976, 98, 1454-1461.

179 D. L. S. Brahms, W. P. Dailey, Chem. Rev., 1996, 96, 1585-1632.

180 D. Barton, Holis, in 'Comprehensive Organic Chemistry'.

181 J. G. Topliss, M. H. Sherlock, F. H. Clarke, M. C. Daly, B. W. Pettersen, J. Lipski, N. Sperber, J. Org. Chem., 1961, 26, 3842-3850. 
182 E. T. McBee, Proc. Indiana Acad. Sci., 1954, 64, 108-109.

183 H. F. Koch, J. A. J. Kielbania, J. Am. Chem. Soc., 1970, 92, 729-730.

184 J. G. A. Kooyman, H. P. A. Hendricks, P. P. Montijn, L. Brandsma, J. F. Arens, Recl. Trav. Chim. Pays-Bas, 1968, 87, 69-72.

185 K. Tanaka, S. Shiraishi, T. Nakia, N. Ishikawa, Tetrahedron Lett., 1978, 34, 3103-3106.

186 A. Bou, M. A. Pericas, F. Serratosa, Tetrahedron, 1981, 37, 1441-1449.

187 M. J. Brown, Heterocycles, 1989, 29, 2225-2294.

188 W. F. Edgell, L.Parts, J. Am. Chem. Soc., 1955, 77, 4899-4901.

189 G. V. D. Tiers, H. A. Brown, T. S. Reid, J. Am. Chem. Soc., 1953, 75, 59785979.

190 P. L. Coe, M. S. Lennard, J. C. Tatlow, J. Fluorine Chem., 1996, 80, 86-90.

191 D. E. Young, L. R. Anderson, D. E. Gould, W. B. Fox, J. Am. Chem. Soc., 1970, 92, 2313-2316.

192 D. Crich, Q. Yao, G. F. Filzen, J. Am. Chem. Soc., 1995, 117, 11455-11470.

193 M. Cavicchioli, A. Mele, V. Montanari, G. Resnai, J. Chem. Soc., Chem. Commun., 1995, 901-902.

194 T. Ogawa, S. Nakabayashi, K. Sasjima, Carbohydr. Res., 1981, 95, 308-312.

195 R. Kuhn, H. H. Baer, Ann., 1958, 611, 236-238.

196 J. J. Nieuwenhuis, J. H. Jordaan, Carbohydr. Res., 1976, 207, 208-213.

197 R. Baumann, A. D. Proud, D. Uhrin, S. L. Flitsch, J. Chem. Soc., Perkin Trans. I., In Preparation.

198 R. Roy, H. J. Jennings, Carbohydr. Res., 1983, 112, 63-72.

199 Z. Zhang, G. Magnusson, Carbohydr. Res., 1996, 295, 41-55.

200 E. B. Rathbone, A. M. Stephen, K. G. R. Pachler, Carbohydr. Res., 1971, 20, 357-367.

201 P. Girad, H. Kagan, Tetrahedron, 1971, 27, 5911-5920. 
202 P. J. Archbald, M. D. Fenn, A. B. Roy, Carbohydr. Res., 1981, 93, 177-190. 


\section{Appendix 1: Publications.}




\title{
Development of a Protecting Group for Sulfate Esters
}

\author{
Andrew D. Proud, Jeremy C. Prodger' and Sabine L. Flitsch* \\ *Department of Chemistry, The University of Edinburgh, King's Buildings. West Mains Road, Edinburgh EH9 3JJ, UK. \\ "GlaxoWellcome, Medicines Research Centre, Gunnels Wood Road, Stevenage, Herrs, SG1 2NY, UK.
}

\begin{abstract}
The trifluoroethyl ester was studied as a protection group for sulfate monoesters in carbohydrates. The ester was formed from the sulfate by treatment with trifluorodiazoethane and was compatible with other common protecting groups used in carbohydrate chemistry. Selective cleavage of the trifluoroethyl ester was achieved with potassium t-butoxide. 1997 Elsevier Science Lid.
\end{abstract}

Biochemical studies are increasingly implicating sulfated oligosaccharides as key structures in important biological interactions. This has led to a growing interest in their use as preliminary targets for pharmaceutical investigation. Although there are a wide range of methods available to sulfate a hydroxyl group, few are suitable for use with complex sugar structures and those that are, generate anionic sulfates. Free sulfates and their salts are difficult to handle and to characterise and hence sulfation is usually carried out as one of the last steps in a synthesis. A protecting group which was stable to conditions usually used in carbohydrate synthesis would clearly be useful for extending synthetic strategies for sulfated oligosaccharides and might also find applications in preparations of other natural products such as sulfated peptides.

An obvious way to mask the anionic nature of the sulfate is to form the sulfate diester (Fig 1.). Such giesters are normally unstable and highly susceptible to nucleophilic attack, which can occur at any one of three positions (a)-(c) (Fig. 1').<smiles>[R]COS(=O)(=O)O[C@H](C)CC[3H]</smiles>

Figure 1: Nucleophilic attack on sulfate diester.

Substitution by route (a) is generally slow when $R$ is a carbohydrate, in particular for the sulfate esters of secondary alcohols. The problem therefore remains to choose $R$ ' such that it disfavours attack by route (b) but still contains a reactive handle to allow selective removal to unmask the sulfate monoester. Perlin er $a l^{2}$ have described the use of phenyl sulfates, which can be hydrogenated to a cyclohexyl sulfate which is then susceptible to base hydrolysis. Unfortunately, both formation of the sulfate diester and cleavage only give moderate yields and the reaction conditions are incompatible with protecting groups that are sensitive to base and to hydrogenation.

We have therefore investigated the use of trihaloethyl sulfate esters, which would be deactivated to nucleophilic attack as in (b) and (c) for both steric and electronic reasons. Our initial attempts focused on trichloroethyl sulfate esters of carbohydrates. Trichloroethyl esters have been used as phosphate and carboxyl protection and can be selectively removed using $\mathrm{Zn} / \mathrm{AcOH}$. Unfortunately, we found that yields 
of synthesis were very poor using a range of reaction conditions, possibly due to steric hindrance. Thus, instead we concentrated on trifluoroethyl sulfate esters. Attempts to introduce both the sulfate and the protection in one step by reacting the free alcohol groups of sugars with 1,1,1-trifluoroethyl chlorosulfate failed to give the desired product and instead yielded a number of unwanted by-products. However, the trifluoroethyl ester was successfully formed directly from the sulfate by using 2,2,2-trifluorodiazoethane as the reagent. This had previously been successfully used by Meese for the highly selective alkylation of sulfonic acids in preference to carboxylic acids and hydroxyl groups ${ }^{3}$. We have found a similar selectivity for sulfate esters. 2,2,2-Trifluorodiazoethane could safely be prepared from trifluoroethylamine ${ }^{t}$ in reasonable quantities ( 40 mmole) s $^{s}$ and was used to esterify a number of sulfated monosaccharides in reasonable to good yields (Table 1).

Table 1: Formation of Trifluoroethyl Esters of Carbohydrate Sulfates.

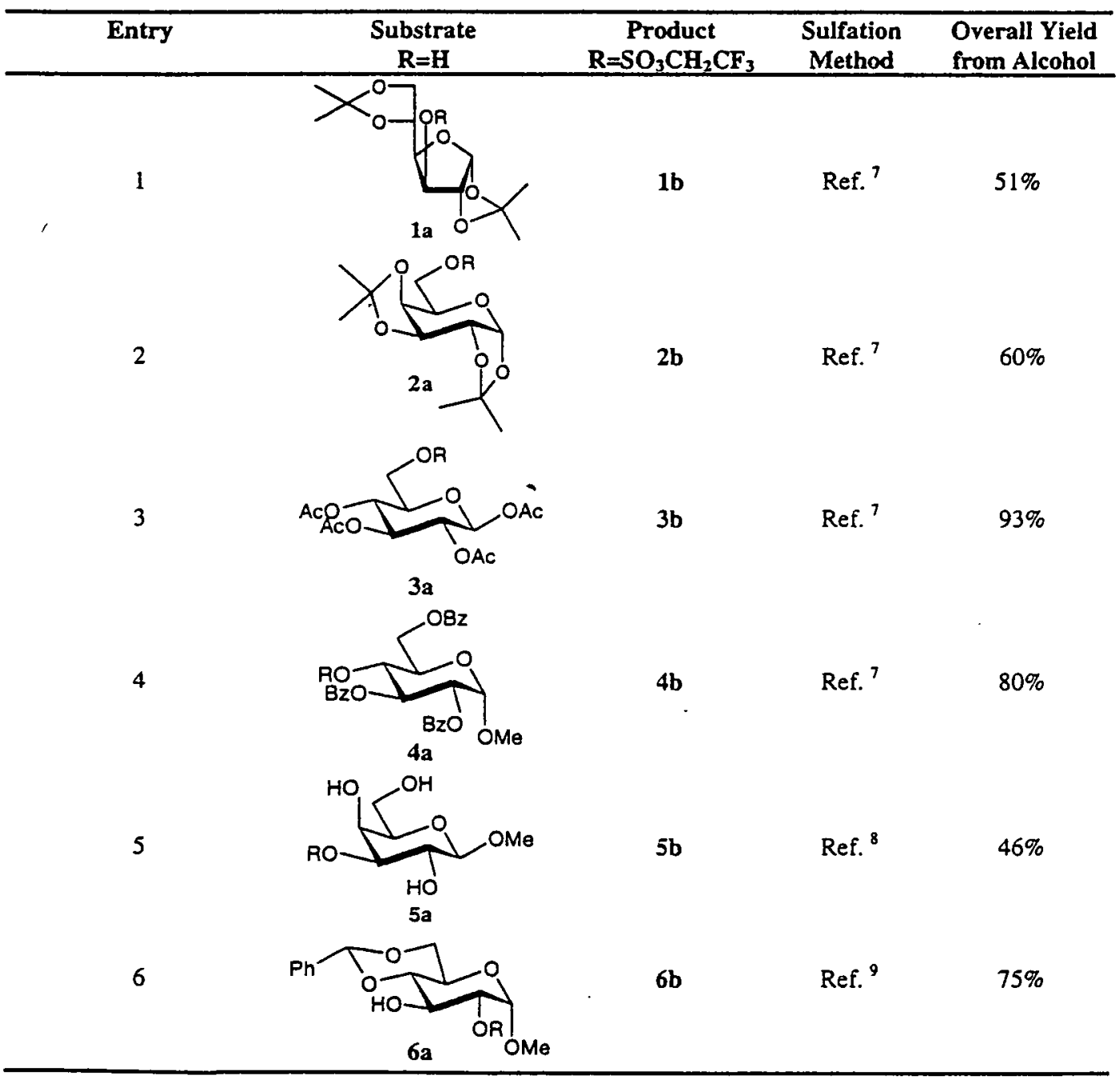


The intermediate sulfates were directly prepared from the partially protected carbohydrates 1a-6a and used without purification often allowing one pot synthesis of the fully protected sulfates $1 b-6 b^{10}$.

Table 2: Deprotected Monosaccharides.

\begin{tabular}{|c|c|c|c|c|c|c|}
\hline Entry & Substrate & $\begin{array}{c}\text { Deprotected } \\
\text { Sugar } \\
\mathrm{R}=\mathrm{SO}_{1} \mathrm{CH}_{2} \mathrm{CF}_{1}\end{array}$ & Method & Yield & $\begin{array}{c}\text { Deprotected } \\
\text { Sulfate } \\
\mathrm{R}=\mathrm{SO}_{1}\end{array}$ & Yield \\
\hline 1 & 1b & & $\begin{array}{c}4: 1 \\
\text { TFA:EtOH } \\
25^{\circ} \mathrm{C} \\
2 \mathrm{hrs}\end{array}$ & $75 \%$ & $1 c^{12}$ & $82 \%$ \\
\hline 2 & $2 b$ & & $\begin{array}{c}4: 1 \\
\text { TFA:EtOH } \\
25^{\circ} \mathrm{C} \\
2 \mathrm{hrs}\end{array}$ & $97 \%$ & $2 c^{12}$ & $96 \%$ \\
\hline 3 & $3 b$ & $9 b$ & $\begin{array}{c}\mathrm{NaOMe} \\
\mathrm{MeOH} \\
2 \mathrm{hr}\end{array}$ & $66 \%$ & $9 c^{13}$ & $88 \%$ \\
\hline
\end{tabular}

The stability of the trifluoroethyl protecting group was then investigated. The $2^{\prime}, 2^{\prime}, 2^{\prime}$ trifluoroethyl sulfate esters were found to be stable to a variety of conditions commonly used to remove carbohydrate protecting groups (see Table 2). The esters were stable to strong organic acids (Table 2; entries 1 and 2) but not to mineral acids such as dilute sulfuric acid (the entire sulfate moiety was cleaved). The protecting group appeared to be stable to hydrogenation conditions (the 4,6-O-Benzylidene of $6 \mathrm{~b}$ was removed in $73 \%$ yield), was thermally stable (refluxing methanol) and was not cleaved when treated with TBAF.

The 2',2',2'-trifluoroethyl ester was surprisingly stable to sodium methoxide in methanol (Table 2; entry 3) such that acetate groups could be selectively removed under Zemplen conditions even at reflux. On the other hand, refluxing in potassium tert butoxide in tert butyl alcohol lead to the removal of the sulfate protecting group in good yields (Table 2). A possible mechanism involves attack of butoxide at the sulfur of the sulfate (see Fig. 1; Route (c)) displacing 2,2,2-trifluoroethoxide, followed by elimination to release 2-methylpropene. The potassium salt of the sulfated sugars $1 \mathrm{c}, 2 \mathrm{c}$ and $9 \mathrm{c}$ could be isolated with the minimum of purification and without the need for ion exchange resins. However deprotection of compounds $5 \mathrm{~b}$ and $\mathbf{6 b}$ resulted in some isomerisation of the sulfate ester, which is currently being investigated.

In conciusion, the 2',2',2'-trifluoroethyl ester seems to be a useful protecting group for sulfate esters, which can easily be formed from the sulfate monoester and can be cleaved in good yields, while being orthogonal to a number of common carbohydrate protecting groups.

Acknowledgements

We are grateful to the EPSRC and GlaxoWellcome for financial support. 


\section{References and Notes:}

1. Kaiser, E. T. The Organic Chemistry of Sulfur, Oae, S.; Plenum Press: New York and London, 1944; pp. 48-74.

2. Penney, C.L.; Perlin, A.S. Carbohydr. Research 1981, 93, 241-246.

3. Meese, C. O. Synthesis 1984, 1041-1042.

4. Ho, J.; Fishbein, J. C. J. Am. Chem. Soc. 1994, 116, 6611-6621.

5. Preparation of 2,2,2-trifluorodiazoethane: CAUTION:- This compound is potentially explosive and must be considered as highly toxic. All experiments using this reagent were carried out in glassware with Clear-Seal joints and behind blast screens:- Sodium Nitrite $(3.0 \mathrm{~g}$, leq) in water $(10 \mathrm{ml})$ was added to a solution of $2,2,2$-trifluoroethylamine hydrochloride $(5.4 \mathrm{~g}, 1 \mathrm{eq})$ in water $(20 \mathrm{ml})$ at $0^{\circ} \mathrm{C}$. A stream of Nitrogen gas was bubbled through the liquid, the mixture of gas and 2,2,2-trifluorodiazoethane was passed over $\mathrm{KOH}$ pellets before being trapped in acetonitrile $(40 \mathrm{ml})$ at $-40^{\circ} \mathrm{C}$. After $1 \mathrm{hr}$ at $0^{\circ} \mathrm{C}$ the aqueous mixture was warmed to room temp over $1 \mathrm{hr}$. The solution of 2,2,2-trifluorodiazoethane was used without further purification.

6. General method for sulfate protection:- 2,2,2-Trifluorodiazoethane solution (10ml, $-10 \mathrm{eq})$ was added to a solution of the crude monosaccharide $(-150 \mathrm{mg}, 1 \mathrm{eq})$ in acetonitrile $(10 \mathrm{ml})$. After 5 mins citric acid ( $1 \mathrm{~g}$ ) was added. The mixture was then stirred at room temperature for $24 \mathrm{hrs}$, the solvent removed in vacuo and the resulting solid was dissolved in water. The mixture was extracted with diethyl ether and the combined organic layers were washed with water, dried $\left(\mathrm{MgSO}_{4}\right)$ and the solvent removed in vacuo. The product was purified by column chromatography.

7. General sulfation method:- The sugar (1.0eq) and pyridine/sulfur trioxide complex (1.0-5.0eq) were heated $\left(80^{\circ} \mathrm{C}\right)$ in acetonitrile until no starting material remained (1-2 hrs).

8. Guilbert, B.; Davis, N. J.; Pearce, M.; Aplin, R. T.; Flitsch, S. L. Tetrahedron: Asymmetry 1994, 5, 2163-2178.

9. Langston, S.; Bernot, B.; Vasella, A. Helv. Chim. Acta. 1994, 77, 2341-2353.

10. Selected data for trifluoroethyl protected monosaccharide sulfates: 1b; HRMS: $\mathrm{C}_{14} \mathrm{H}_{22} \mathrm{~F}_{3} \mathrm{O}_{9} \mathrm{~S}$ requires 423.09367, found 423.09308. 2b; HRMS: $\mathrm{C}_{14} \mathrm{H}_{22} \mathrm{~F}_{3} \mathrm{O}_{9} \mathrm{~S}$ requires 423.09367 , found 423.09357. 3b; Anal. Calcd for $\mathrm{C}_{16} \mathrm{H}_{21} \mathrm{~F}_{3} \mathrm{O}_{13} \mathrm{~S}: \mathrm{C}, 37.65 ; \mathrm{H}, 4.15 \%$. Found: C, 37.62; $\mathrm{H}, 4.21 \%$. $4 \mathrm{~b}$; Anal. Calcd for $\mathrm{C}_{30} \mathrm{H}_{27} \mathrm{~F}_{3} \mathrm{O}_{12} \mathrm{~S}: \mathrm{C}, 53.89 ; \mathrm{H}, 4.07 \%$, Found: C, 54.04; H, 4.21\%. 5b; ${ }^{1} \mathrm{H}-\mathrm{NMR}$ $\left(250 \mathrm{MHz}, \mathrm{CD}_{3} \mathrm{OD}\right): \delta 3.65(1 \mathrm{H}$, ddd, $J=6.6,5.5,0.9 \mathrm{~Hz}, 5-\mathrm{H}), 3.81(3 \mathrm{H}, \mathrm{s}, \mathrm{OMe}), 3.82(1 \mathrm{H}, \mathrm{dd}$, $J=9.9,7.6 \mathrm{~Hz}, 2-\mathrm{H}), 4.30(1 \mathrm{H}, \mathrm{dd}, J=3.3,0.9 \mathrm{~Hz}, 4-\mathrm{H}), 4.31(1 \mathrm{H}, \mathrm{d}, J=7.6 \mathrm{~Hz}, 1-\mathrm{H}), 4.64(1 \mathrm{H}$, dd, $J=9.9,3.3 \mathrm{~Hz}, 3-\mathrm{H}), 4.91\left(2 \mathrm{H}, \mathrm{q}, J=8.1 \mathrm{~Hz}, \mathrm{CH}_{2} \mathrm{CF}_{3}\right) 4.83-5.06(2 \mathrm{H}, \mathrm{m}, 6-\mathrm{Ha}, 6-\mathrm{Hb}) .{ }^{13} \mathrm{C}-$ NMR $\left(250 \mathrm{MHz}, \mathrm{CD}_{3} \mathrm{OD}\right): \delta 55.58,59.99,66.03,66.13\left(\mathrm{q}, J=37.9 \mathrm{~Hz}, \mathrm{CH}_{2} \mathrm{CF}_{3}\right) 67.86,73.76$, $86.68,103.70,121.68\left(\mathrm{q}, J=276.4 \mathrm{~Hz}, C \mathrm{~F}_{3}\right) .{ }^{19} \mathrm{~F}-\mathrm{NMR}\left(250 \mathrm{MHz}, \mathrm{CD}_{3} \mathrm{OD}\right): \delta-78.30(3 \mathrm{~F}, \mathrm{~s}) .6 \mathrm{~b}$; HRMS: $\mathrm{C}_{16} \mathrm{H}_{20} \mathrm{~F}_{3} \mathrm{O}_{9} \mathrm{~S}$ requires 445.07802 , found 445.07860 .

11. General method for deprotection of trifluoroethylsulfate esters:- Substrate (10mg, leq) was dissolved in tert butyl alcohol, potassium tert butoxide (5eq) was added and mixture refluxed until reaction was complete ( $1-2 \mathrm{hrs}$ ). The product was purified by column chromatography.

12. Mumma, R. O.; Hoiberg, C.P.;Simpson, R. Carbohydr. Res. 1970, 14, 119-121.

13. Lupescu, N.; Arad, S.; Geresh, S.; Bernstein, M. A.; Glaser, R. Carbohydr. Res. 1991, 210, 349352.

(Received in UK 13 June 1997; revised 11 August 1997; accepted 15 August 1997) 


\section{Appendix 2: X-Ray Data.}


X-Ray Crystallography data:

Phenyl $\quad 3,4,6$-tri- $O$-acetyl- $\alpha-D$-thioglucopyranoside $\quad 2-\left(2^{\prime}, 2^{\prime}, 2^{\prime}\right.$-trifluoroethyl sulfate) (54).

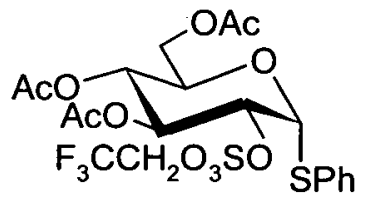

(54)

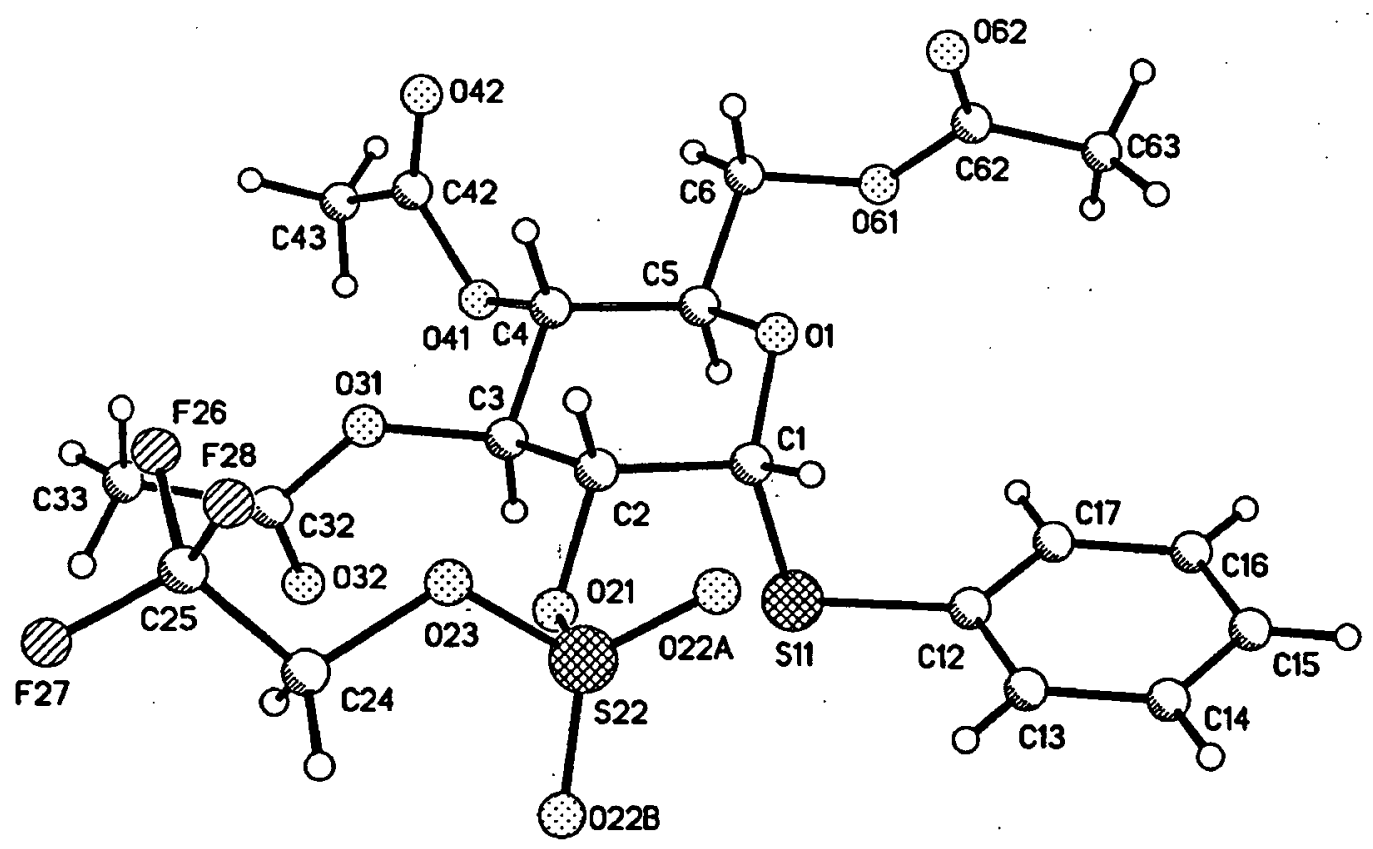


Table 1. Crystal data and structure refinement for xap578.

a. CRystat DAta

Empirical formula
Formula weight
Wavelength
Temperature
Crystal system
Space group
Unit cell dimensions

Volume

Number of reflections for cell

$\mathbf{z}$

Density (calculated)

Absorption coefficient

$F(000)$

B. DATA COLIECTION

Crystal description

Crystal size

Theta range for data collection

Index ranges

Reflections collected

Independent reflections

scan type

Absorption correction

C. SOLUTION AND REFINEMENT.

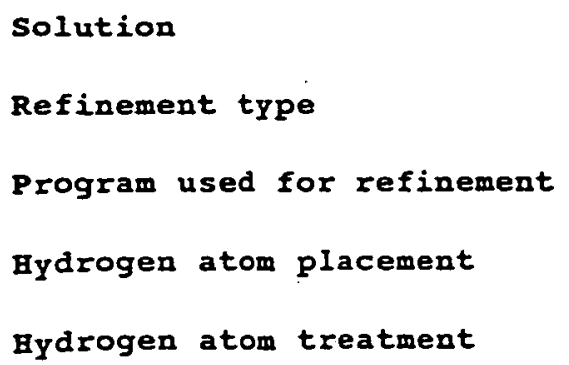

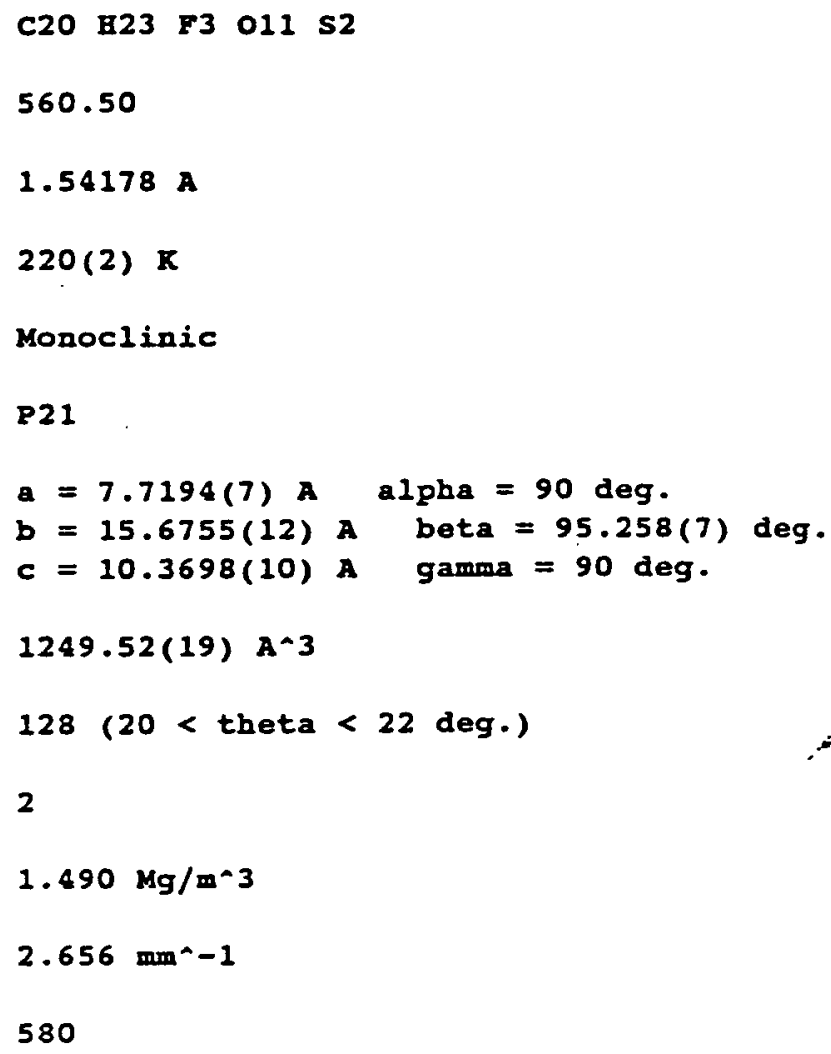


Table 2. Atomic coordinates $\left(x 10^{-4}\right)$ and equivalent isotropic displacement parameters (A^2 $\times 10 \wedge 3$ ) for xap578. U(eq) is defined as one third of the trace of the orthogonalized Uij tensor.

\begin{tabular}{|c|c|c|c|c|}
\hline & $x$ & $\mathbf{Y}$ & $\mathbf{z}$ & $v(e q)$ \\
\hline$O(1)$ & $1147(2)$ & $-1552(1)$ & $263(2)$ & $29(1)$ \\
\hline$c(1)$ & $1817(3)$ & $-1162(2)$ & $1432(2)$ & $27(1)$ \\
\hline$s(11)$ & $3927(1)$ & $-1577(1)$ & $2097(1)$ & $35(1)$ \\
\hline$c(12)$ & $5258(4)$ & $-976(2)$ & $1124(3)$ & $32(1)$ \\
\hline$c(13)$ & $5753(4)$ & $-157(2)$ & $1497(3)$ & $44(1)$ \\
\hline$c(14)$ & $6788(5)$ & $328(3)$ & $776(4)$ & $57(1)$ \\
\hline$c(15)$ & $7373(4)$ & $-24(3)$ & $-326(4)$ & $58(1)$ \\
\hline$c(16)$ & $6906(5)$ & $-833(3)$ & $-719(4)$ & $55(1)$ \\
\hline$c(17)$ & $5825(4)$ & $-1318(2)$ & $14(3)$ & $42(1)$ \\
\hline$c(2)$ & $447(4)$ & $-1239(2)$ & $2406(3)$ & $26(1)$ \\
\hline $0(21)$ & $1090(3)$ & $-907(1)$ & $3670(2)$ & $31(1)$ \\
\hline$s(22)$ & $.964(1)$ & $77(1)$ & $3915(1)$ & $31(1)$ \\
\hline$O(22 A)$ & $1193(3)$ & $525(2)$ & $2763(2)$ & $46(1)$ \\
\hline$O(22 B)$ & $2018(3)$ & $223(2)$ & $5079(2)$ & $43(1)$ \\
\hline$o(23)$ & $-993(3)$ & $197(2)$ & $\$ 139(2)$ & $38(1)$ \\
\hline$c(24)$ & $-1422(4)$ & $132(3)$ & $5472(3)$ & $44(1)$ \\
\hline$c(25)$ & $-3343(5)$ & $209(3)$ & $5415(3)$ & $49(1)$ \\
\hline$F(26)$ & $-4155(4)$ & $-445(2)$ & $4779(3)$ & $86(1)$ \\
\hline$F(27)$ & $-3876(3)$ & $204(3)$ & $6565(2)$ & $98(1)$ \\
\hline$F(28)$ & $-3947(3)$ & $887(2)$ & $4772(3)$ & $75(1)$ \\
\hline$c(3)$ & $-15(4)$ & $-2163(2)$ & $2611(3)$ & $27(1)$ \\
\hline$o(31)$ & $-1483(3)$ & $-2185(1)$ & $3371(2)$ & $33(1)$ \\
\hline$c(32)$ & $-1308(4)$ & $-2634(2)$ & $4495(3)$ & $33(1)$ \\
\hline$O(32)$ & $-6(3)$ & $-3006(2)$ & $4871(2)$ & $45(2)$ \\
\hline$c(33)$ & $-2938(5)$ & $-2610(3)$ & $5158(4)$ & $49(1)$ \\
\hline$c(4)$ & $-559(4)$ & $-2580(2)$ & $1308(3)$ & $28(1)$ \\
\hline$O(41)$ & $-749(3)$ & $-3480(1)$ & $1561(2)$ & $33(1)$ \\
\hline$c(42)$ & $-2271(4)$ & $-3850(2)$ & $1101(3)$ & $39(1)$ \\
\hline$O(42)$ & $-3321(5)$ & $-3482(2)$ & $386(4)$ & $93(1)$ \\
\hline$c(43)$ & $-2378(5)$ & $-4750(2)$ & $1534(3)$ & $45(1)$ \\
\hline$c(5)$ & $799(4)$ & $-2445(2)$ & $362(3)$ & $30(1)$ \\
\hline$c(6)$ & $171(4)$ & $-2741(2)$ & $-988(3)$ & $35(1)$ \\
\hline$o(61)$ & $1553(3)$ & $-2627(2)$ & $-1809(2)$ & $37(1)$ \\
\hline$c(62)$ & $1399(5)$ & $-1996(2)$ & $-2699(3)$ & $40(1)$ \\
\hline $0(62)$ & $122(3)$ & $-1551(2)$ & $-2880(2)$ & $89(1)$ \\
\hline$c(63)$ & $2977(5)$ & $-1920(3)$ & $-3390(3)$ & $53(1)$ \\
\hline
\end{tabular}


Table 3. Bond lengths [A] and angles [deg] for xap578.

\begin{tabular}{|c|c|}
\hline$O(1)-c(1)$ & $1.413(3)$ \\
\hline$O(1)-C(5)$ & $1.432(3)$ \\
\hline$c(1)-c(2)$ & $1.533(4)$ \\
\hline$c(1)-s(11)$ & $1.830(3)$ \\
\hline$s(11)-c(12)$ & $1.776(3)$ \\
\hline$c(12)-c(17)$ & $1.378(5)$ \\
\hline$c(12)-c(13)$ & $1.384(5)$ \\
\hline$c(13)-c(14)$ & $1.372(5)$ \\
\hline$c(14)-c(15)$ & $1.382(7)$ \\
\hline$c(15)-c(16)$ & $1.371(7)$ \\
\hline$c(16)-c(17)$ & $1.402(5)$ \\
\hline$c(2)-0(21)$ & $1.455(3)$ \\
\hline$c(2)-c(3)$ & $1.511(4)$ \\
\hline$O(21)-5(22)$ & $1.568(2)$ \\
\hline$S(22)-O(22 A)$ & $1.411(2)$ \\
\hline$S(22)-O(22 B)$ & $1.412(2)$ \\
\hline$s(22)-0(23)$ & $1.561(2)$ \\
\hline$O(23)-c(24)$ & $1.454(4)$ \\
\hline$c(24)-c(25)$ & $1.483(5)$ \\
\hline$C(25)-F(27)$ & $1.297(4)$ \\
\hline$C(25)-F(28)$ & $1.318(5)$ \\
\hline$C(25)-F(26)$ & $1.342(5)$ \\
\hline$c(3)-0(31)$ & $1.438(3)$ \\
\hline$c(3)-c(4)$ & $1.525(4)$ \\
\hline$O(31)-c(32)$ & $1.358(8)$ \\
\hline$c(32)-0(32)$ & $1.196(4)$ \\
\hline$c(32)-c(33)$ & $1.489(5)$ \\
\hline$C(4)-0(41)$ & $1.444(3)$ \\
\hline$c(4)-c(5)$ & $1.515(4)$ \\
\hline$O(41)-c(42)$ & $1.357(4)$ \\
\hline$c(42)-0(42)$ & $1.195(5)$ \\
\hline$c(42)-c(43)$ & $1.485(5)$ \\
\hline$c(5)-c(6)$ & $1.511(4)$ \\
\hline$c(6)-0(61)$ & $1.436(4)$ \\
\hline$O(61)-c(62)$ & $1.351(4)$ \\
\hline$c(62)-0(62)$ & $1.208(4)$ \\
\hline$c(62)-c(63)$ & $1.473(5)$ \\
\hline$c(1)-0(1)-c(5)$ & $114.7(2)$ \\
\hline$o(1)-c(1)-c(2)$ & $108.1(2)$ \\
\hline$o(1)-c(1)-s(11)$ & $114.38(19)$ \\
\hline$c(2)-c(1)-s(11)$ & $111.56(18)$ \\
\hline$c(12)-s(11)-c(1)$ & $97.92(13)$ \\
\hline$c(17)-c(12)-c(13)$ & $119.6(3)$ \\
\hline$c(17)-c(12)-s(11)$ & $120.8(3)$ \\
\hline$c(13)-c(12)-s(11)$ & $119.6(2)$ \\
\hline$c(14)-c(13)-c(12)$ & $121.4(4)$ \\
\hline$c(13)-c(14)-c(15)$ & $118.7(4)$ \\
\hline$c(16)-c(15)-c(14)$ & $121.2(4)$ \\
\hline$c(15)-c(16)-c(17)$ & $119.6(4)$ \\
\hline$c(12)-c(17)-c(16)$ & $119.5(4)$ \\
\hline$o(21)-c(2)-c(3)$ & $106.4(2)$ \\
\hline$o(21)-c(2)-c(1)$ & $111.5(2)$ \\
\hline$c(3)-c(2)-c(1)$ & $110.8(2)$ \\
\hline$c(2)-0(21)-s(22)$ & $118.46(17)$ \\
\hline$O(22 A)-S(22)-O(22 B)$ & $122.45(16)$ \\
\hline$O(22 A)-S(22)-O(23)$ & $105.32(14)$ \\
\hline$O(22 B)-S(22)-O(23)$ & $110.06(12)$ \\
\hline$O(22 A)-S(22)-O(21)$ & $109.81(13)$ \\
\hline$O(22 B)-S(22)-O(21)$ & $104.93(14)$ \\
\hline
\end{tabular}




$\begin{array}{ll}O(23)-S(22)-O(21) & 102.62(13) \\ C(24)-O(23)-S(22) & 116.17(18) \\ O(23)-C(24)-C(25) & 105.7(3) \\ F(27)-C(25)-F(28) & 110.0(4) \\ F(27)-C(25)-F(26) & 105.9(4) \\ F(28)-C(25)-F(26) & 103.9(3) \\ F(27)-C(25)-C(24) & 111.3(3) \\ F(28)-C(25)-C(24) & 113.0(3) \\ F(26)-C(25)-C(24) & 112.2(3) \\ O(31)-C(3)-C(2) & 107.9(2) \\ O(31)-C(3)-C(4) & 108.0(2) \\ C(2)-C(3)-C(4) & 109.6(2) \\ C(32)-O(31)-C(3) & 117.5(2) \\ O(32)-C(32)-O(31) & 123.2(3) \\ O(32)-C(32)-C(33) & 125.7(3) \\ O(31)-C(32)-C(33) & 111.2(3) \\ O(41)-C(4)-C(5) & 110.0(2) \\ O(41)-C(4)-C(3) & 106.4(2) \\ C(5)-C(4)-C(3) & 111.3(2) \\ C(42)-O(41)-C(4) & 116.9(2) \\ O(42)-C(42)-O(41) & 121.9(3) \\ O(42)-C(42)-C(43) & 126.3(3) \\ O(41)-C(42)-C(43) & 111.7(3) \\ O(1)-C(5)-C(6) & 106.2(2) \\ O(1)-C(5)-C(4) & 109.2(2) \\ C(6)-C(5)-C(4) & 112.0(2) \\ O(61)-C(6)-C(5) & 108.6(2) \\ C(62)-O(61)-C(6) & 118.1(2) \\ O(62)-C(62)-O(61) & 123.3(3) \\ O(62)-C(62)-C(63) & 125.2(3) \\ O(61)-C(62)-C(63) & 111.5(3) \\ & \end{array}$

Symmetry transformations used to generate equivalent atoms: 
Table 4. Anisotropic displacement parameters $\left(A^{-2} \times 10^{\wedge 3}\right)$ for xap578. The anisotropic displacement factor exponent takes the form:

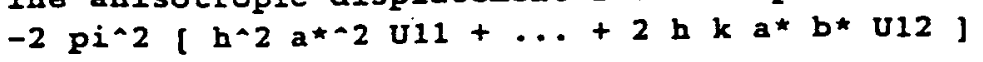

\begin{tabular}{|c|c|c|c|c|c|c|}
\hline & U11 & $\mathrm{U} 22$ & v33 & U23 & v13 & U12 \\
\hline $\begin{array}{l}0(1) \\
c(1)\end{array}$ & $\begin{array}{l}35(1) \\
29(1)\end{array}$ & $24(1)$ & $28(1)$ & $1(1)$ & $4(1)$ & $-1(1)$ \\
\hline $5(11)$ & $29(1)$ & $36(1)$ & $38(1)$ & $10(1)$ & $3(1)$ & $-1(1)$ \\
\hline$c(12)$ & $24(1)$ & $37(2)$ & $35(1)$ & 7 (1) & $1(1)$ & $1(1)$ \\
\hline$c(13)$ & $40(2)$ & $46(2)$ & $47(2)$ & $-1(2)$ & $2(1)$ & $-10(2)$ \\
\hline$c(14)$ & $46(2)$ & $42(2)$ & $81(3)$ & $12(2)$ & $-5(2)$ & $-13(2)$ \\
\hline$c(15)$ & $35(2)$ & $66(3)$ & $72(3)$ & $32(2)$ & $8(2)$ & $-4(2)$ \\
\hline$c(16)$ & $46(2)$ & $74(3)$ & $50(2)$ & $18(2)$ & $23(2)$ & $24(2)$ \\
\hline$c(17)$ & $42(2)$ & $39(2)$ & $46(2)$ & $8(1)$ & $9(1)$ & $15(2)$ \\
\hline$c(2)$ & $29(1)$ & $24(1)$ & $26(1)$ & $-2(1)$ & $4(1)$ & $2(1)$ \\
\hline$O(21)$ & $41(1)$ & $23(1)$ & $28(1)$ & $-1(1)$ & $4(1)$ & $1(1)$ \\
\hline$s(22)$ & $36(1)$ & $25(1)$ & $34(1)$ & $-4(1)$ & $6(1)$ & $-1(1)$ \\
\hline$O(22 A)$ & $66(2)$ & $27(1)$ & $46(1)$ & $4(1)$ & $16(1)$ & $-1(1)$ \\
\hline$O(22 B)$ & $39(1)$ & $41(1)$ & $48(1)$ & $-12(1)$ & $1(1)$ & $-4(1)$ \\
\hline$o(23)$ & $36(1)$ & $40(1)$ & $37(1)$ & $-2(1)$ & $3(1)$ & $6(1)$ \\
\hline$c(24)$ & $42(2)$ & $48(2)$ & $42(2)$ & $7(2)$ & $8(1)$ & $4(2)$ \\
\hline$c(25)$ & $47(2)$ & $55(2)$ & $48(2)$ & $8(2)$ & $13(1)$ & $9(2)$ \\
\hline$F(26)$ & $55(1)$ & $85(2)$ & $116(2)$ & $-8(2)$ & $-1(1)$ & $-21(2)$ \\
\hline$F(27)$ & $59(1)$ & $175(4)$ & $62(1)$ & $20(2)$ & $26(1)$ & $16(2)$ \\
\hline$F(28)$ & $58(1)$ & $72(2)$ & $96(2)$ & $24(2)$ & $20(1)$ & $33(1)$ \\
\hline$c(3)$ & $26(1)$ & $22(1)$ & $33(1)$ & $2(1)$ & $9(1)$ & $0(1)$ \\
\hline$O(31)$ & $30(1)$ & $31(1)$ & $39(1)$ & $8(1)$ & $13(1)$ & $O(1)$ \\
\hline$c(32)$ & $43(2)$ & $23(1)$ & $35(1)$ & $1(1)$ & $8(1)$ & $-5(1)$ \\
\hline $0(32)$ & $52(1)$ & $41(1)$ & $43(1)$ & $12(1)$ & $11(1)$ & $6(1)$ \\
\hline$c(33)$ & $52(2)$ & $46(2)$ & $52(2)$ & $8(2)$ & $20(2)$ & $-2(2)$ \\
\hline$c(4)$ & $31(1)$ & $18(1)$ & $35(1)$ & $2(1)$ & $2(1)$ & $-1(1)$ \\
\hline$O(41)$ & $36(1)$ & $20(1)$ & $41(1)$ & $4(1)$ & $2(1)$ & -5 (1) \\
\hline$c(42)$ & $41(2)$ & $31(2)$ & $45(2)$ & $O(1)$ & $-3(1)$ & $-10(1)$ \\
\hline$O(42)$ & $74(2)$ & $48(2)$ & $145(3)$ & $26(2)$ & $-56(2)$ & $-22(2)$ \\
\hline$c(43)$ & $55(2)$ & $32(2)$ & $47(2)$ & $-1(2)$ & $2(1)$ & $-16(2)$ \\
\hline$c(5)$ & $34(1)$ & $22(1)$ & $33(1)$ & $O(1)$ & $3(1)$ & $1(1)$ \\
\hline$c(6)$ & $40(2)$ & $33(2)$ & $32(1)$ & $-3(1)$ & $5(1)$ & $0(1)$ \\
\hline$O(61)$ & $44(1)$ & $34(1)$ & $34(1)$ & $O(1)$ & $8(1)$ & $7(1)$ \\
\hline$c(62)$ & $52(2)$ & $32(2)$ & $34(1)$ & $-5(1)$ & $1(1)$ & $4(2)$ \\
\hline$O(62)$ & $58(1)$ & $41(1)$ & $47(1)$ & $7(1)$ & $5(1)$ & $13(1)$ \\
\hline$c(63)$ & $53(2)$ & $60(2)$ & $48(2)$ & $8(2)$ & $10(2)$ & $-2(2)$ \\
\hline
\end{tabular}


Table 5. Bydrogen coordinates $(\times 10-4)$ and isotropic displacement parameters (A^2 2 10.3) for xap578.

\begin{tabular}{|c|c|c|c|c|}
\hline & $x$ & $Y$ & $z$ & $\mathbf{u}(\mathbf{e q})$ \\
\hline H(1) & 1969 & -547 & 1253 & 32 \\
\hline $\mathbf{H}(13)$ & 5372 & 72 & 2260 & 53 \\
\hline B (14) & 7094 & 888 & 1028 & 68 \\
\hline$B(15)$ & 8105 & 298 & -815 & 69 \\
\hline B(16) & 7306 & -1062 & -1475 & 66 \\
\hline $\mathrm{B}(17)$ & 5491 & -1873 & -250 & 50 \\
\hline$B(2)$ & -612 & -922 & 2078 & 31 \\
\hline$E(24 A)$ & -861 & 591 & 5999 & 52 \\
\hline$B(24 B)$ & -1038 & -417 & 5847 & 52 \\
\hline $\mathbf{B}(3)$ & 985 & -2470 & 3061 & 32 \\
\hline$B(33 A)$ & -3809 & -2277 & 4645 & 73 \\
\hline $\mathrm{B}(33 \mathrm{~B})$ & -2705 & -2351 & 6005 & 73 \\
\hline B (33C) & -3364 & -3187 & 5254 & 73 \\
\hline E (4) & -1687 & -2342 & 944 & 34 \\
\hline$E(43 A)$ & -1392 & -4877 & 2152 & 67 \\
\hline $\mathrm{B}(43 \mathrm{~B})$ & -2363 & -5127 & 792 & 67 \\
\hline$E(43 C)$ & -3448 & -4834 & 1939 & 67 \\
\hline$E(5)$ & 1881 & -2751 & 668 & 35 \\
\hline$E(6 A)$ & -165 & -3343 & -971 & 42 \\
\hline$A(6 B)$ & -848 & $-2 ₫ 07$ & -1320 & 42 \\
\hline $\mathrm{E}(63 \mathrm{~A})$ & 3819 & -2345 & -3065 & 80 \\
\hline $\mathrm{E}(63 \mathrm{~B})$ & 3472 & -1355 & -3250 & 80 \\
\hline$B(63 C)$ & 2678 & -2009 & -4309 & 80 \\
\hline
\end{tabular}




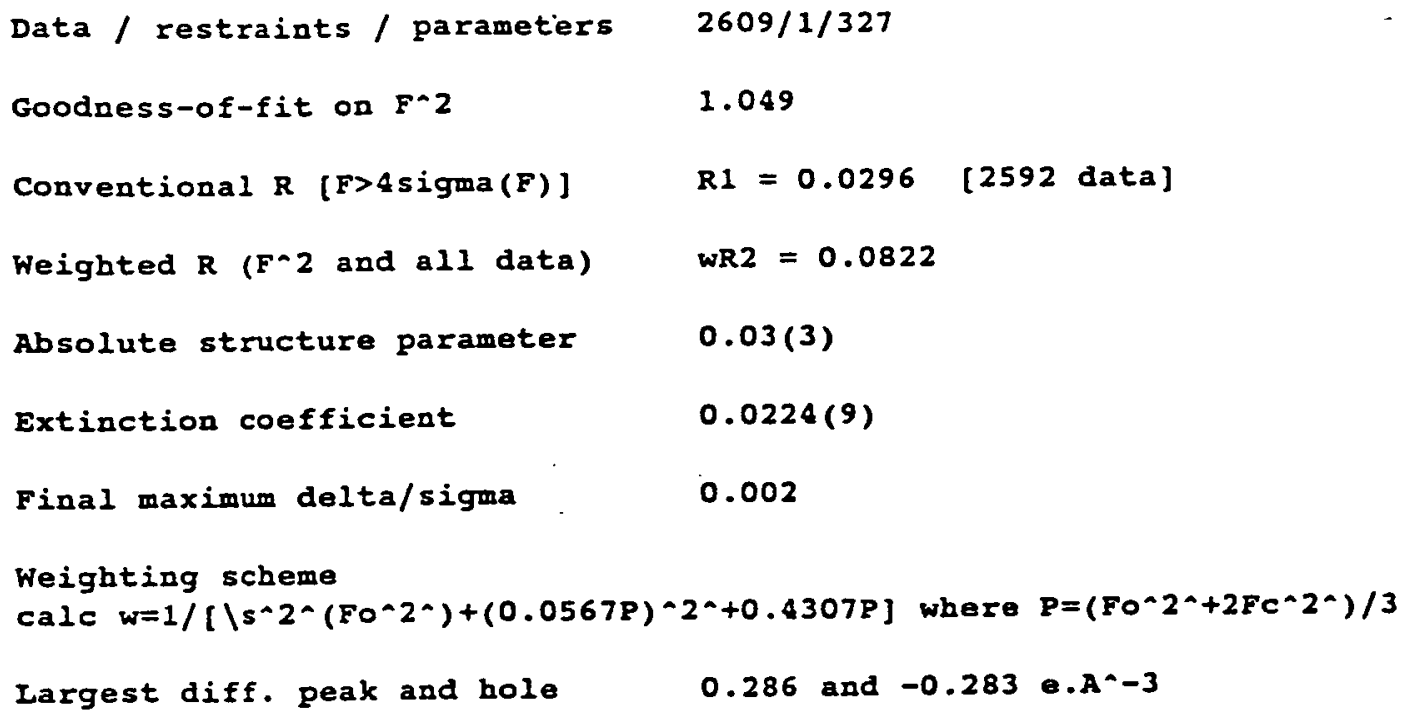


X-Ray Crystallography data:

6-Deoxy-1,2:3,4-di- $O$-isopropylidene-D-galacto-5-hexenoaldo-1,5-pyranose (90).

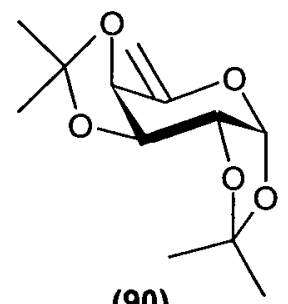

(90)

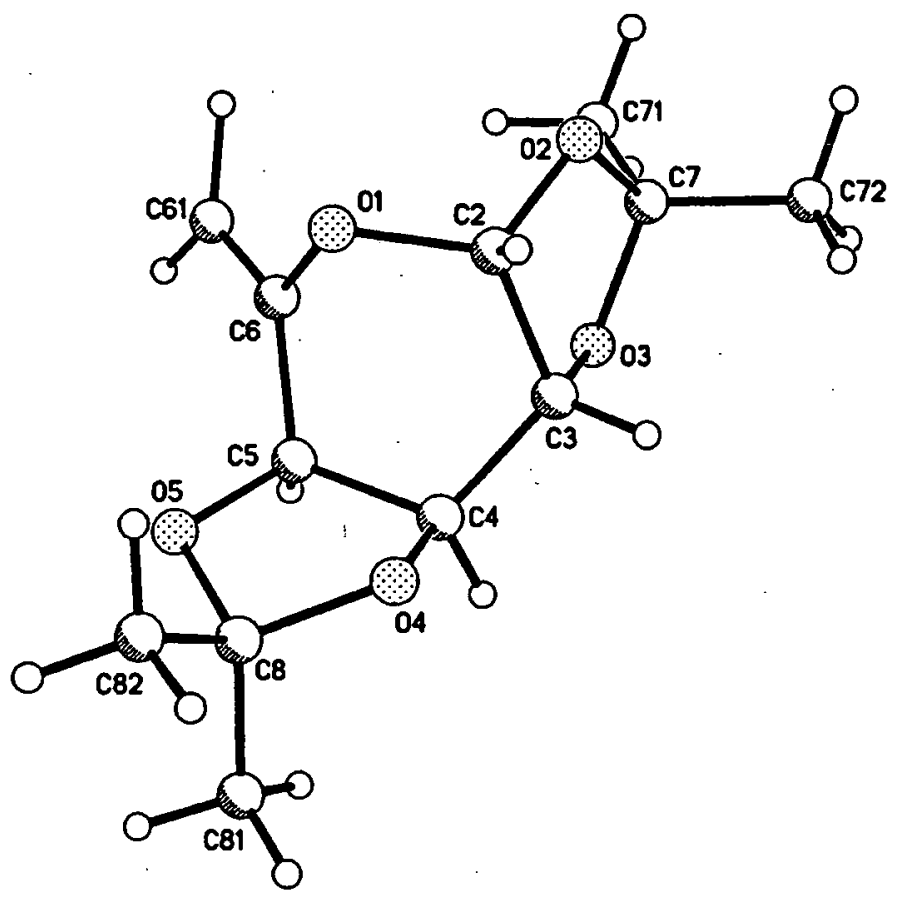


Table 1. Crystal data and structure refinement for apr558.

A. CRYStar data

Empirical formula

Formula weight

Wavelength

Temperature

Crystal systan

Space group

Unit cell dimensions

Volume

Number of reflections for cell

z

Density (calculated)

Absorption coefficient

$\mathbf{F}(000)$

B. DATA COLHection

Crystal description

Crystal size

Theta range for data collection

Index ranges

Reflections collected

Independent reflections

Scan type

C. SOLUTION AND REPINEMENT.

\section{solution}

Refinement type

Program used for refinement

Bydrogen atom placement

Bydrogen atom treatment

Data / restraints / parameters
C12 11805

242.26

$1.54184 \mathrm{~A}$

$220(2) \times$

Monoclinic

P21

$\mathrm{a}=5.630(3) \mathrm{A} \quad \mathrm{alpha}=90 \mathrm{deg}$.

$\mathrm{b}=10.617(6) \mathrm{A}$ beta $=97.69(5) \mathrm{deg}$.

$c=10.372(8)$ A gama $=90 \mathrm{deg}$.

$614.4(7) A-3$

$50(25<$ theta $<30 \mathrm{deg} \cdot)$

2

$1.310 \mathrm{Mg} / \mathrm{m}^{\wedge} 3$

$0.850 m-1$

260

colourless plate developed in (001)

$0.38 \times 0.31 \times 0.04 \mathrm{~mm}$

4.30 to $70.19 \mathrm{deg}$.

$-6<=h<=6,-1<=k<=12,-1<=1<=12$

2899

$1259[R($ int $)=0.0418$ ]

Omega-theta

direct (sHer.s S-97)

Full-matrix least-squares on Fa2

SBEILXI-97

geometric/difference map

riding/refall (=CE2)

$1259 / 1 / 167$ 


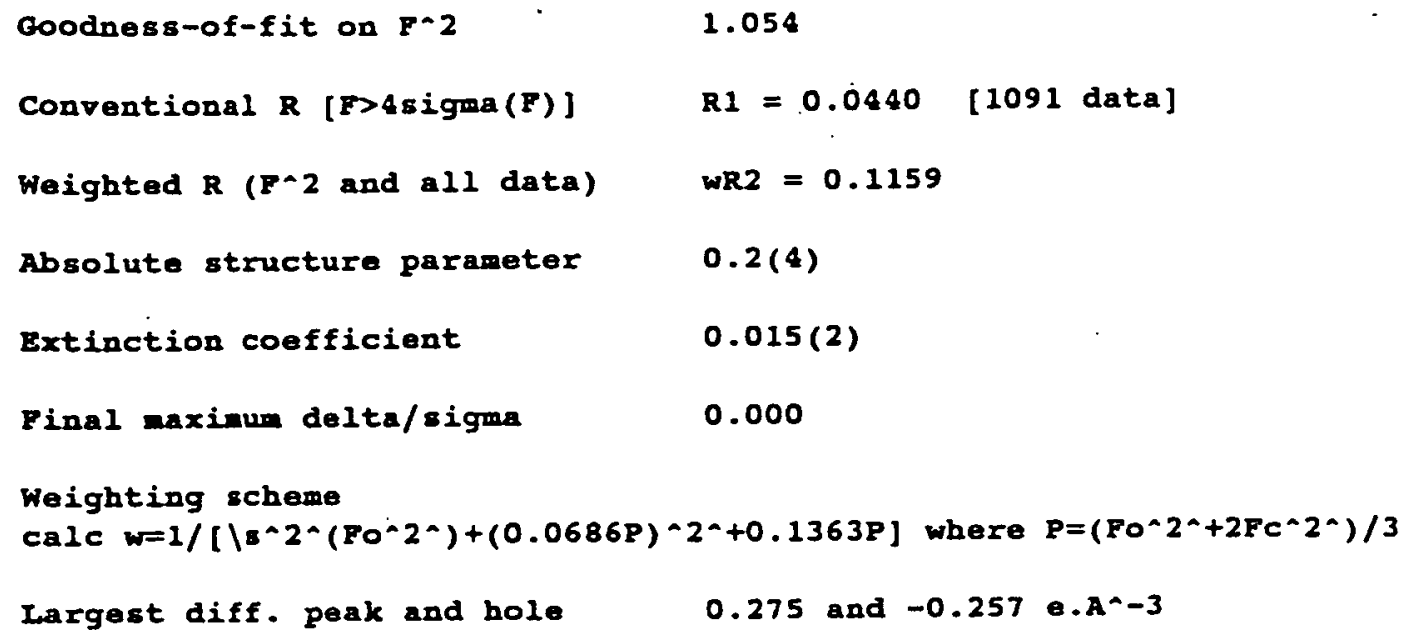


Table 2. Atomic coordinates $\left(\times 10^{-4}\right)$ and equivalent isotropic displacement parameters (A-2 $\times 10-3)$ for apr558. U(eq) is defined as one third of the trace of the orthogonalized Uij tensor.

\begin{tabular}{|c|c|c|c|c|}
\hline & $x$ & $Y$ & $\mathbf{x}$ & $\mathrm{U}(\mathrm{eq})$ \\
\hline$O(1)$ & $9963(4)$ & $6168(2)$ & $6638(3)$ & $37(1)$ \\
\hline$c(2)$ & $10607(6)$ & $5375(4)$ & $5635(3)$ & $32(1)$ \\
\hline$c(3)$ & $9510(7)$ & $4061(4)$ & $5620(3)$ & $33(1)$ \\
\hline$c(4)$ & $8949(6)$ & $3613(4)$ & $6922(3)$ & $32(1)$ \\
\hline$c(5)$ & $7634(6)$ & $4600(4)$ & $7640(3)$ & $33(1)$ \\
\hline$c(6)$ & $7796(5)$ & $5905(4)$ & $7089(3)$ & $31(1)$ \\
\hline$O(2)$ & $9751(4)$ & $5852(3)$ & $4406(2)$ & $39(1)$ \\
\hline$o(3)$ & $7308(4)$ & $4224(3)$ & $4788(2)$ & $33(1)$ \\
\hline$c(7)$ & $7741(6)$ & $5108(4)$ & $3808(3)$ & $33(1)$ \\
\hline$c(71)$ & $5586(6)$ & $5936(5)$ & $3496(4)$ & $44(1)$ \\
\hline$c(72)$ & $8473(7)$ & $4445(5)$ & $2633(4)$ & $44(1)$ \\
\hline$O(4)$ & $11145(4)$ & $3426(3)$ & $7748(2)$ & $38(1)$ \\
\hline$O(5)$ & $8863(4)$ & $4584(3)$ & $8940(2)$ & $41(1)$ \\
\hline$c(8)$ & $10636(7)$ & $3602(5)$ & $9047(4)$ & $40(1)$ \\
\hline$C(81)$ & $9628(10)$ & $2426(6)$ & $9588(4)$ & $57(1)$ \\
\hline$c(82)$ & $12853(7)$ & $4087(6)$ & $9867(4)$ & $57(1)$ \\
\hline$c(61)$ & $6156(7)$ & $6791(5)$ & $7093(4)$ & $42(1)$ \\
\hline
\end{tabular}


Table 3. Bond lengths [A] and angles [deg] for apr558.

\begin{tabular}{|c|c|}
\hline$O(1)-c(6)$ & $1.392(4)$ \\
\hline$o(1)-c(2)$ & $1.423(5)$ \\
\hline$c(2)-o(2)$ & $1.396(4)$ \\
\hline$c(2)-c(3)$ & $1.525(6)$ \\
\hline$c(3)-o(3)$ & $1.423(4)$ \\
\hline$c(3)-c(4)$ & $1.504(5)$ \\
\hline$c(4)-0(4)$ & $1.420(4)$ \\
\hline$c(4)-c(5)$ & $1.532(5)$ \\
\hline$c(5)-0(5)$ & $1.431(4)$ \\
\hline$c(5)-c(6)$ & $1.507(6)$ \\
\hline$c(6)-c(61)$ & $1.318(6)$ \\
\hline$o(2)-c(7)$ & $1.450(4)$ \\
\hline$o(3)-c(7)$ & $1.428(4)$ \\
\hline$c(7)-c(71)$ & $1.498(5)$ \\
\hline$c(7)-c(72)$ & $1.512(5)$ \\
\hline$o(4)-c(8)$ & $1.427(5)$ \\
\hline$O(5)-c(8)$ & $1.438(5)$ \\
\hline$c(8)-c(82)$ & $1.504(6)$ \\
\hline$c(8)-c(81)$ & $1.510(7)$ \\
\hline$c(6)-0(1)-c(2)$ & $116.6(3)$ \\
\hline$O(2)-c(2)-O(1)$ & $111.3(3)$ \\
\hline$o(2)-c(2)-c(3)$ & $103.5(3)$ \\
\hline$o(1)-c(2)-c(3)$ & $114.0(3)$ \\
\hline$o(3)-c(3)-c(4)$ & $108.2(3)$ \\
\hline$o(3)-c(3)-c(2)$ & $102.3(3)$ \\
\hline$c(4)-c(3)-c(2)$ & $114.5(3)$ \\
\hline$o(4)-c(4)-c(3)$ & $108.4(3)$ \\
\hline$o(4)-c(4)-c(5)$ & $103.9(3)$ \\
\hline$c(3)-c(4)-c(5)$ & $113.2(3)$ \\
\hline$o(5)-c(5)-c(6)$ & $108.7(3)$ \\
\hline$o(5)-c(5)-c(4)$ & $104.3(3)$ \\
\hline$c(6)-c(5)-c(4)$ & $112.8(3)$ \\
\hline$c(61)-c(6)-O(1)$ & $120.2(4)$ \\
\hline$c(61)-c(6)-c(5)$ & $125.3(3)$ \\
\hline$o(1)-c(6)-c(5)$ & $114.3(3)$ \\
\hline$c(2)-o(2)-c(7)$ & $110.3(3)$ \\
\hline$c(3)-o(3)-c(7)$ & $107.1(3)$ \\
\hline$o(3)-c(7)-o(2)$ & $104.6(3)$ \\
\hline$o(3)-c(7)-c(71)$ & $109.2(3)$ \\
\hline$o(2)-c(7)-c(71)$ & $109.6(3)$ \\
\hline$o(3)-c(7)-c(72)$ & $111.0(3)$ \\
\hline$o(2)-c(7)-c(72)$ & $108.5(3)$ \\
\hline$c(71)-c(7)-c(72)$ & $113.5(3)$ \\
\hline$c(4)-o(4)-c(8)$ & $106.4(3)$ \\
\hline$c(5)-0(5)-c(8)$ & $108.9(3)$ \\
\hline$o(4)-C(8)-O(5)$ & $104.3(3)$ \\
\hline$O(4)-C(8)-C(82)$ & $108.8(3)$ \\
\hline$o(5)-c(8)-c(82)$ & $108.1(4)$ \\
\hline$o(4)-c(8)-c(81)$ & $112.1(4)$ \\
\hline$o(5)-c(8)-c(8 I)$ & $109.7(4)$ \\
\hline$c(82)-c(8)-c(81)$ & $113.4(4)$ \\
\hline
\end{tabular}

Symmetry transformations used to generate equivalent atoms: 
Table 4. Anisotropic displacement parameters $(A-2 \times 10 \cdot 3)$ for apr558. The anisotropic displacement factor exponent takes the form:

$-2 p i \wedge 2\left[h^{\wedge} 2 a * \wedge 2 u 11+\ldots+2 h k a * b \star u 12\right]$

\begin{tabular}{|c|c|c|c|c|c|c|}
\hline & 011 & $\mathbf{u} 22$ & U33 & $\mathbf{U} 23$ & $\mathbf{U 1 3}$ & $\mathrm{U} 12$ \\
\hline$O(1)$ & $35(1)$ & $28(2)$ & $50(2)$ & $-5(1)$ & $9(1)$ & $-6(1)$ \\
\hline$c(2)$ & $31(2)$ & $31(2)$ & $34(2)$ & $1(2)$ & $5(1)$ & $-2(2)$ \\
\hline$c(3)$ & $40(2)$ & $26(2)$ & $32(2)$ & $-2(2)$ & $3(1)$ & $O(2)$ \\
\hline$c(4)$ & $41(2)$ & $26(2)$ & $28(2)$ & $1(2)$ & $2(1)$ & $-1(2)$ \\
\hline$c(5)$ & $32(2)$ & $33(2)$ & $34(2)$ & $-1(2)$ & $6(1)$ & $1(2)$ \\
\hline$c(6)$ & $27(2)$ & $32(2)$ & $35(2)$ & $-6(2)$ & $4(1)$ & $-4(2)$ \\
\hline$o(2)$ & $35(1)$ & $38(2)$ & $42(1)$ & $11(1)$ & $-1(1)$ & $-9(1)$ \\
\hline$o(3)$ & $37(1)$ & $32(2)$ & $30(1)$ & $4(1)$ & $1(1)$ & $-8(1)$ \\
\hline$c(7)$ & $31(2)$ & $36(2)$ & $33(2)$ & $8(2)$ & $3(1)$ & $-5(2)$ \\
\hline$c(71)$ & $34(2)$ & $51(3)$ & $46(2)$ & $12(2)$ & $2(2)$ & $1(2)$ \\
\hline$c(72)$ & $49(2)$ & $49(3)$ & $36(2)$ & $4(2)$ & $9(2)$ & $1(2)$ \\
\hline$O(4)$ & $44(2)$ & $43(2)$ & $28(1)$ & $-3(1)$ & $3(1)$ & $12(1)$ \\
\hline$O(5)$ & $47(1)$ & $45(2)$ & $32(1)$ & $-3(1)$ & $5(1)$ & $13(2)$ \\
\hline$c(8)$ & $45(2)$ & $44(3)$ & $31(2)$ & $-4(2)$ & $5(2)$ & $9(2)$ \\
\hline$C(81)$ & $92(4)$ & $39(3)$ & $41(2)$ & $6(2)$ & $10(2)$ & $1(3)$ \\
\hline$c(82)$ & $46(2)$ & $85(4)$ & $39(2)$ & $-6(3)$ & $O(2)$ & $8(3)$ \\
\hline$c(61)$ & $39(2)$ & $34(2)$ & $53(2)$ & $-4(2)$ & $6 .(2)$ & $6(2)$ \\
\hline
\end{tabular}


Table 5. Hydrogen coordinates ( $\left.\times 10^{4} 4\right)$ and isotropic displacement parameters (A^2 $\times 10^{\wedge} 3$ ) for apr558.

\begin{tabular}{|c|c|c|c|c|}
\hline & $x$ & $\mathbf{Y}$ & z & $U(e q)$ \\
\hline $\mathbf{E}(2)$ & 12374 & 5303 & 5721 & 38 \\
\hline E(3) & 10542 & 3444 & 5243 & 39 \\
\hline H (4) & 8019 & 2819 & 6824 & 38 \\
\hline B (5) & 5936 & 4355 & 7635 & 39 \\
\hline$E(71 A)$ & 5292 & 6386 & 4273 & 66 \\
\hline $\mathrm{B}(71 \mathrm{~B})$ & 4202 & 5423 & 3186 & 66 \\
\hline$B(71 C)$ & 5869 & 6535 & 2827 & 66 \\
\hline$B(72 A)$ & 7144 & 3943 & 2221 & 67 \\
\hline$B(72 B)$ & 9834 & 3901 & 2904 & 67 \\
\hline $\mathrm{H}(72 \mathrm{C})$ & 8912 & 5065 & 2020 & 67 \\
\hline$E(81 A)$ & 8203 & 2164 & 9021 & 86 \\
\hline$E(81 B)$ & 9216 & 2600 & 10449 & B6 \\
\hline $\mathrm{E}(\mathrm{B1C})$ & 10816 & 1760 & 9642 & 86 \\
\hline $\mathrm{B}(\mathbf{8 2 A})$ & 12520 & 4220 & 10750 & 86 \\
\hline $\mathrm{E}(\mathbf{8 2 B})$ & 13335 & 4877 & 9510 & 86 \\
\hline B (82C) & 14135 & 3476 & 9869 & 86 \\
\hline H(61A) & $4820(80)$ & $6630(60)$ & $7560(40)$ & $58(14)$ \\
\hline $\mathrm{B}(61 \mathrm{~B})$ & $6420(100)$ & $7640(70)$ & $6530(50)$ & $81(18)$ \\
\hline
\end{tabular}

